
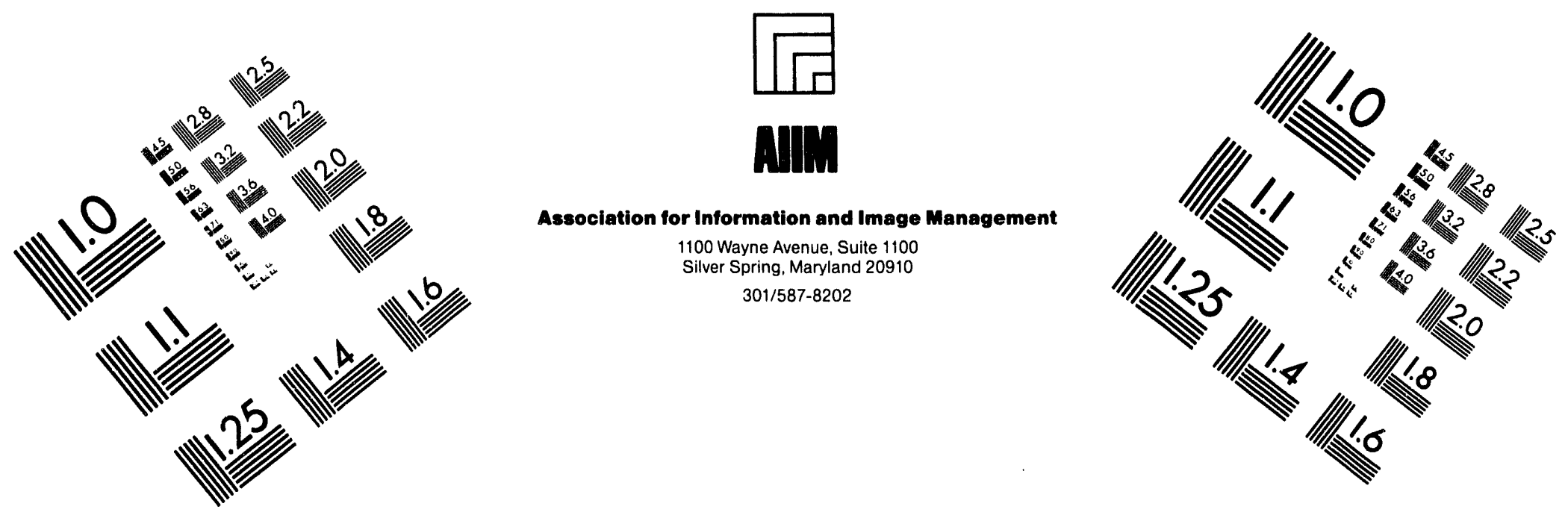

\title{
Centimeter
}

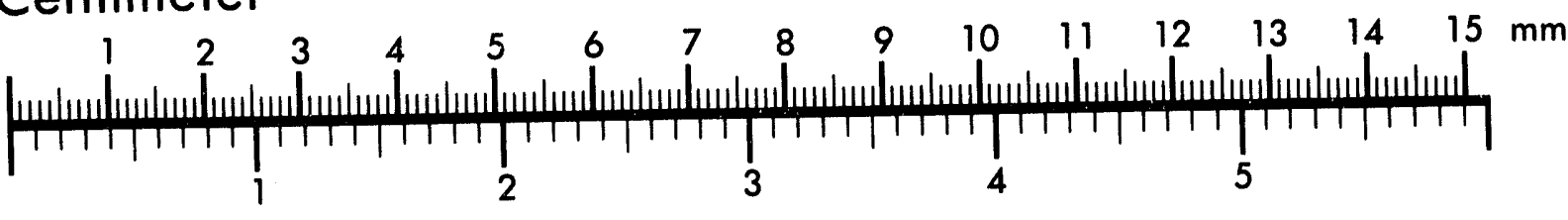
Inches
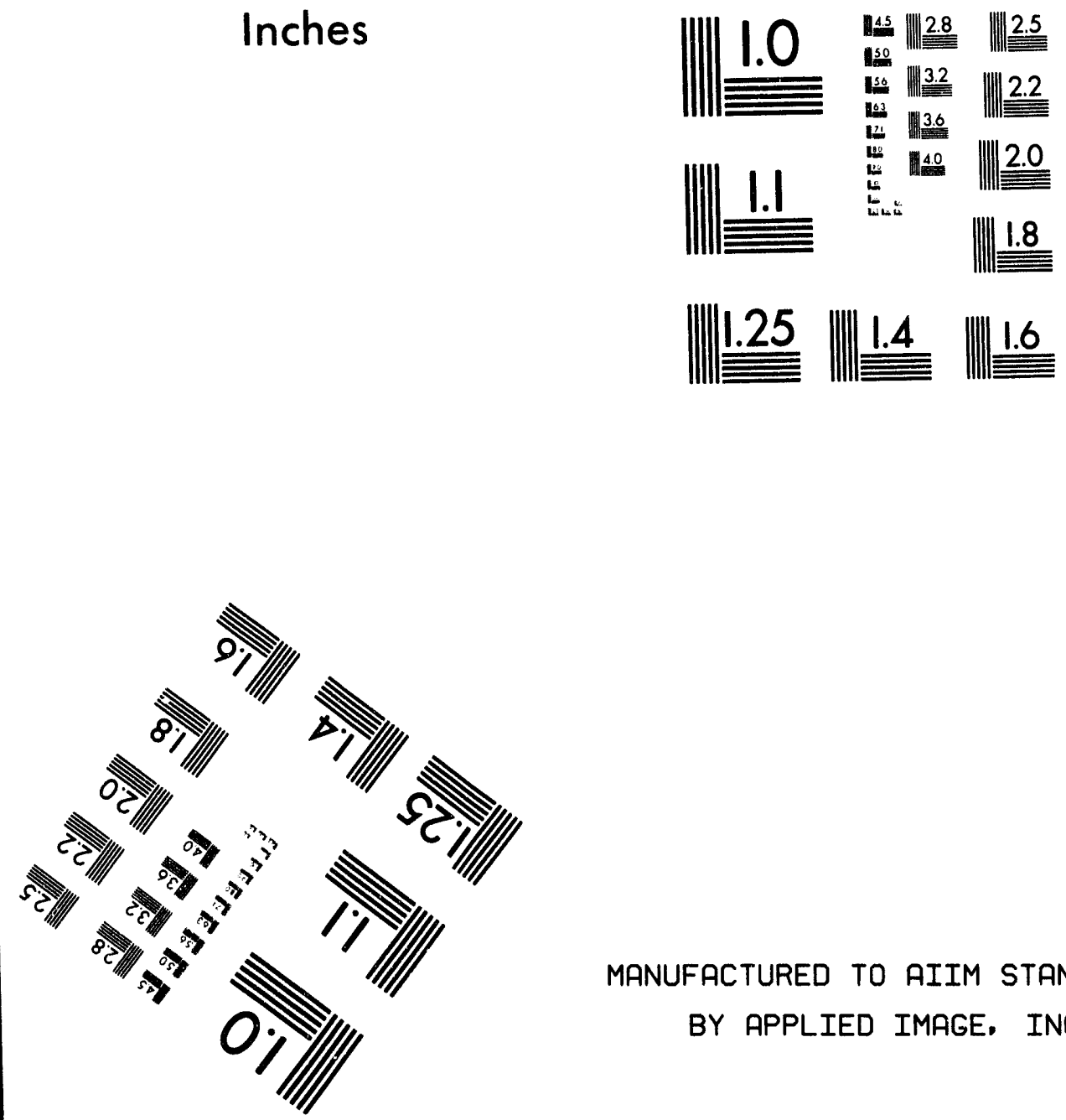

MANUFACTURED TO AIIM STANDARDS

BY APPLIED IMAGE, INC.

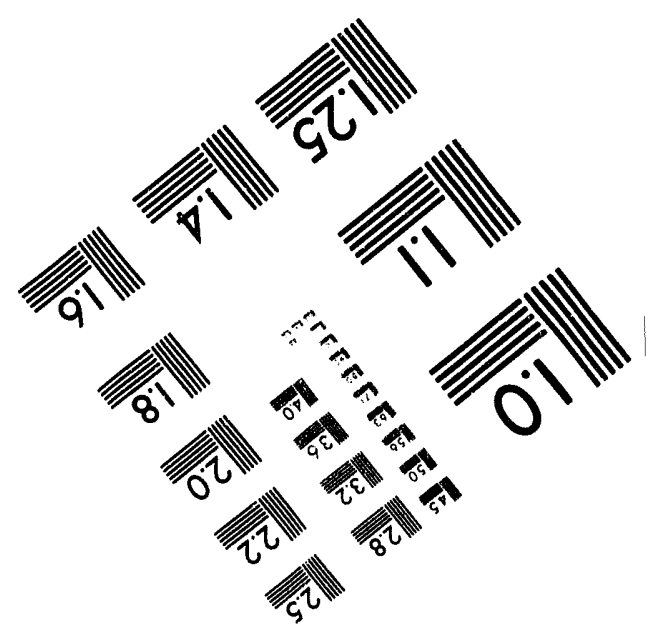



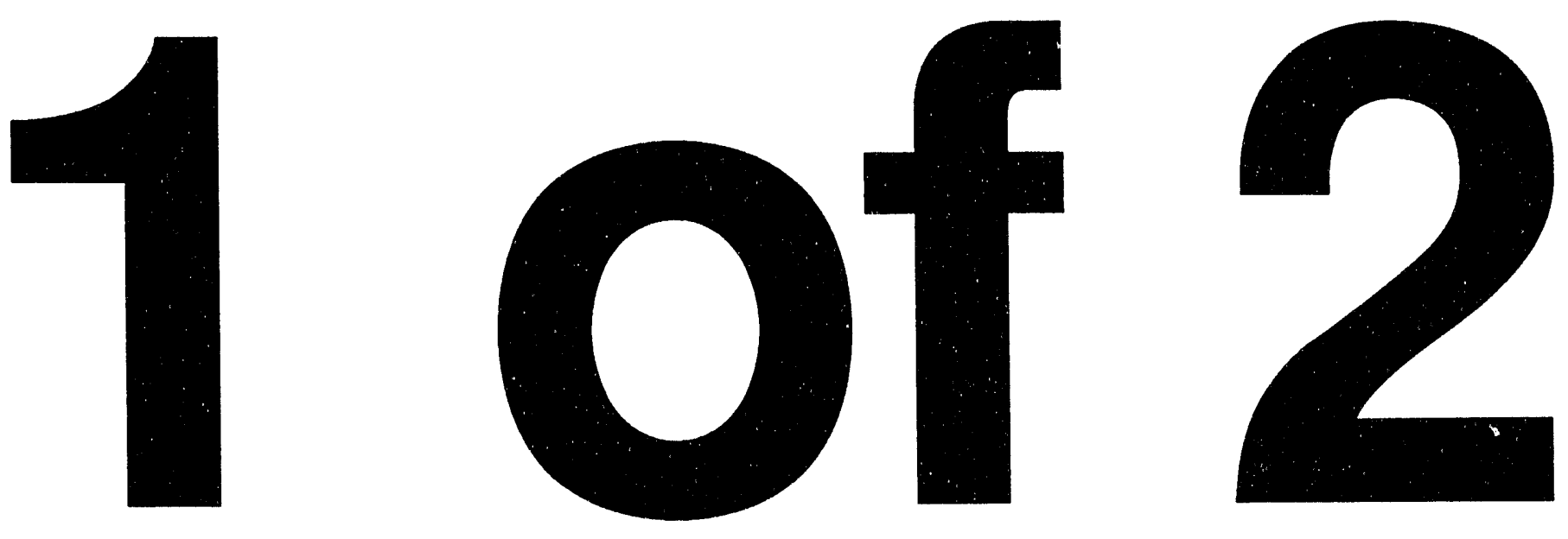


\section{RECURSIVE RECOVERY OF MARKOV TRANSITION PROBABILITIES FROM BOUNDARY VALUE DATA ${ }^{1}$}

\section{Sarah Kathryn Patch}

Department of Mathematics and

Lawrence Berkeley Laboratory

University of California

Berkeley, CA 94720

Ph.D. Thesis

April 1994

1 This work was supported in part by AFOSR under Contract FDF-49620-92-J-0067-11792, by the Applied Mathematical Sciences Subprogram of the Office of Energy Research, U.S. Department of Energy under Contract DE-AC03-76SF00098, by the National Aeronautics and Space Administration under Grant NAG3-1143, and by the National Science Foundation under Grant. DMS89-02831. 


\section{Abstract \\ Recursive Recovery of Markov Transition Probabilities from Boundary Value Data \\ Sarah Kathryn Patch}

In an effort to mathematically describe the anisotropic diffusion of infrared radiation in biological tissue Grünbaum posed an anisotropic diffusion boundary value problem in 1989. In order to accommodate anisotropy, he discretized the temporal as well as the spatial domain. The probabilistic interpretation of the diffusion equation is retained; radiation is assumed to travel according to a random walk (of sorts). In this random walk the probabilities with which photons change direction depend upon their previous as well as present location. The forward problem gives boundary value data as a function of the Markov transition probabilities. The inverse problem requires finding the transition probabilities from boundary value data.

Problems in the plane are studied carefully in this thesis. Consistency conditions amongst the data are derived. These conditions have two effects: they prohibit inversion of the forward map but permit smoothing of noisy data. Next, a recursive algorithm which yields a family of solutions to the inverse problem is detailed. This algorithm takes advantage of all independent data and generates a system of highly nonlinear algebraic equations. Plücker-Graßmann relations are instrumental in simplifying the equations. The algorithm is used to solve the $4 \times 4$ problem. Finally, the smallest nontrivial problem in three dimensions, the $2 \times 2 \times 2$ problem, is solved. 


\section{Contents}

1 Introduction $\quad 1$

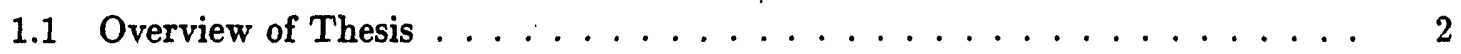

1.2 Brief Overview of Other Imaging Techniques $\ldots \ldots \ldots \ldots 2$

1.3 Description of Diffuse Tomography . . . . . . . . . . . . 3

2 The Direct Problem 6

3 Two Dimensional Problems 13

3.1 Consistency Conditions . . . . . . . . . . . . . . . . . 13

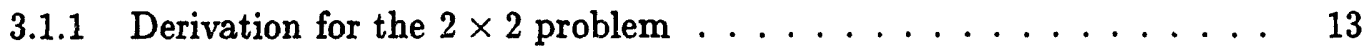

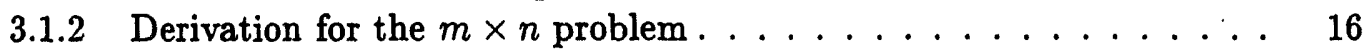

3.1.3 Data subject to Conditions . . . . . . . . . . . . 18

3.1.4 Examples for square systems . . . . . . . . . . . . . . 19

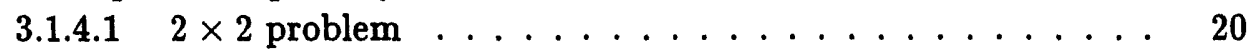

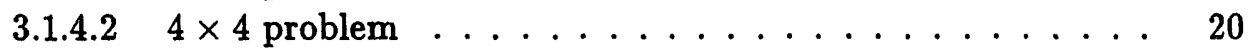

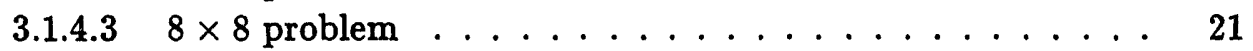

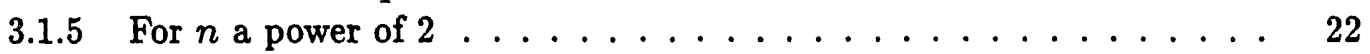

3.2 Recursive Inversion Algorithm . . . . . . . . . . . . . . . . 24

3.2.1 Base Case $(2 \times 2$ problem $) \ldots \ldots \ldots \ldots 24$

3.2.1.1 Solving the Governing Equations . . . . . . . . . . . 24

3.2.1.2 Graßmannians and the Graßmann-Plücker Embedding . . . 30

3.2.1.2.1 Graßmannians ................ 30

3.2.1.2.2 The Plücker Embedding . . . . . . . . . . . 31

3.2.1.2.3 Derivation of Graßmann-Plücker Relations . . . . 32

3.2.1.3 Simplifying solutions to $P_{i h}$ by adding Graßmann identities $\quad 37$

3.2.1.4 A "special" model with a closed system of equations . . . . 39

3.2.1.4.1 Cubics and Quadratics ............ 40

3.2.1.4.2 Simplifying cubics by adding Graßmann identities 46

3.2.1.4.3 Solving the Equations . . . . . . . . . . . . 49

3.2.2 Writing the equations for general $n \ldots \ldots \ldots \ldots$

$3.2 .34 \times 4$ problem . . . . . . . . . . . . . . . 62

3.2.3.1 Solving for $P_{i h}, P_{h h}, P_{i o}$, and $P_{h o}$ in terms of $A \ldots 62$

3.2.3.2 Eliminating $A_{i, j}$ 's $\ldots \ldots \ldots \ldots \ldots$ 
3.2.3.2.1 Identities . . . . . . . . . . . . 73

3.2.3.2.2 Easy Conditions . . . . . . . . . . . 75

3.2.3.2.3 Hard Conditions . . . . . . . . . . . . 84

3.2.3.2.4 Very Hard Conditions . . . . . . . . . . . 89

3.2.4 $n \times n$ problem where $n=2^{k}, k \in \mathbb{N} \ldots \ldots \ldots . \ldots . \ldots 9$

4 Three Dimensional Problems 107

4.1 Introduction . . . . . . . . . . . . . . . . . 107

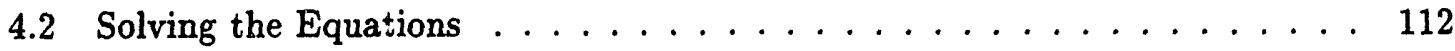

$\begin{array}{lll}5 \text { Conclusion } & 117\end{array}$

$\begin{array}{lr}\text { Bibliography } & 119\end{array}$ 


\section{Chapter 1}

\section{Introduction}

Nearly one century has passed since Röntgen took the first radiograph of his wife's hand. Since that time many different techniques for noninvasive imaging of human tissue have been developed. A concise history of the development of medical imaging can be found in [14]. Some of these techniques are direct descendants of Röntgen's radiograph; others are completely unrelated. Computerized tomography, for example, is a direct descendant of the radiograph. The word "tomography" refers to imaging an object by slices. X rays have high energy and travel straight through the body. Both CT and magnetic resonance imaging, (MRI), permit recovery of an image from knowledge of slices of the object. Data analysis makes use of the Radon transform, which is linear. Ultrasound and impedance imaging are examples of imaging techniques which enjoy neither straight travel paths nor linear inversion formulas. The oxymoron "diffuse tomography" refers to low energy imaging in which the paths of the radiant energy are not necessarily straight and are unknown. Data analysis in diffuse tomography is highly nonlinear and yields a vector valued function. Because it is a low energy technique problems in diffuse tomography are highly nonlinear. Clinical applications such as neonatal imaging and annual mammograms are not amenable to high energy techniques which might overexpose the patient to harmful radiation. Experimentalists in optical tomography work with infrared radiation. Motivated by their work, Grünbaum posed an anisotropic diffusion boundary value problem in 1989. 


\subsection{Overview of Thesis}

This thesis addresses some of the most basic questions in diffuse tomography. Despite the fundamental nature of this work, many of the calulations are quite involved. This section attempts to give the reader a brief road map of the rest of the thesis in order to prevent the reader from becoming lost in a sea of matrices and minors.

In Chapter 2 the forward problem is discussed for the smallest nontrivial two dimensional problem, for larger problems in the plane, and for problems in $d$ dimensions. Chapter 3 concentrates on problems in the plane. It constitutes the bulk of this thesis. Before attempting to solve the iuverse problem, a thorough understanding of the range of the forward map is required. Therefore, consistency conditions amongst the data are studied in 3.1. The goal, of course, is an inversion formula or inversion algorithm. Because of consistency conditions amongst the data it is impossible to invert the forward map. It is possible, however, to find a $p$-parameter family of solutions where $p$ equals the difference between the amount of data and the number of independent consistency conditions. In section 3.2 a recusive recovery scheme which takes full advantage of all of the independent data is detailed. The base case for this algorithm is solved in section 3.2.1. Graßmannians and the Graßmann-Plücker embedding are studied in subsection 3.2.1.2. They are used to simplify the solution to the $2 \times 2$ problem in section 3.2.1.3. The next level of the recusive scheme is handled in section 3.2 .3 , yielding an analytic solution to the $4 \times 4$ problem. In section 3.2.3.2 the number of parameters in the solution found in 3.2.3 is reduced using consistency conditions amongst the data. Consistency conditions and Graßmann relations are used to eliminate more of the parameters in section 3.2.3.2.2. Elimination of parameters continues, in sections 3.2.3.2.3 and 3.2.3.2.4. The reader should be warned that these sections are quite technical. Finally, the smallest nontrivial three dimensional problem is studied in Chapter 4.

\subsection{Brief Overview of Other Imaging Techniques}

To this day, radiographs are one of the most prevalent imaging methods. They are much like photographs, except for the fact that radiographs use higher energy radiation than light to form the image. A radiograph essentially plots an average density function,

$$
\bar{\rho}(x, y)=\int_{\mathbb{R}} \rho(x, y, z) d z
$$


where $\rho$ is the density of the tissue being imaged.

Computerized tomography, (CT), has quickly become one of the mainstays of medical imaging. CT images display tissue density. With the help of intravenous contrast enhancers, CT is capable of providing useful images of soft tissue. It is able to resolve small features extremely well and data collection can be done quickly, reducing blurring due to motion of internal organs.

Positron Emission Tomography, (PET), requires injection of a radioactive label (with a short half life!) into the patient. The isotopes emit positrons which are annihilated by electrons, creating $\gamma$ rays which are measured transaxially. From these measurements the distribution of the label inside the body can be recovered.

Magnetic resonance imaging provides excellent images contrasting hydrogen concentrations and relaxation times of perturbed hydrogen dipoles. MRI is extremely useful for imaging the brain and spinal cord, areas where tissue is soft and CT provides poor resolution unless intravenous contrast enhancers are used.

Real time ultrasound images have become a useful clinical tool, particularly for prenatal imaging. Although data analysis is linearized the images obtained are clear enough to prove diagnostically useful.

Radiographs, CT, and PET assume that the radiating energy/ray travels in a straight line. Although MRI is a completely different technique, in an idealized setting the (Bloch) equations governing the response of hydrogen nuclei are linear. Furthermore, MRI data is often collected for a single "slice" of the object being imaged. In ultrasound and impedance imaging neither electrical currents nor sound waves are assumed to travel in straight lines through the body and in that respect they are somewhat similar to diffuse tomography. In this thesis, however, no approximations or truncations are made to linearize any of the governing equations.

\subsection{Description of Diffuse Tomography}

Experimentalists in optical tomography are presently working with infrared and near infrared radiation as another means of noninvasive imaging. Optical coherence tomography, (OCT), makes use of light waves which are reflected. A beam is directed towards the tissue being imaged. The light enters the tissue and some of it is reflected backwards. By comparing the reflected light to the reference beam, the depth inside the tissue at which 
the light was reflected can be calculated. Another type of optical tomography motivated this work. Another group of experimentalists (Barbour et al, Benaron, Chance et al, Delpy et al, Gratton, ...) use information given by light which passes through tissue. For an introduction to optical imaging see the recent articles [16, 17, 18, 19] and [20]. More detailed research papers can be found in the proceedings [21] and [22]. As photons travel they are scattered many times. This scattering complicates the inverse problem. Therefore, most experimentalists use only data provided by ballistic photons, those which are scattered the least. This thesis gives a detailed study of a Markovian model of photon migration in the plane and a preliminary look at photon migration in three dimensions. Here we consider data generated by all photons, no matter how many scattering events they experience inside the imaging object. We begin by describing the transport model in two dimensions.

Consider an $n \times n$ array of pixels in the plane which covers the object to be reconstructed. On each of the $4 n$ outer edges there are two devices. One device shoots photons across the outside edge into the neighboring pixel; the other device detects photons as they leave the system. For each of the $4 n$ outside edges $4 n$ pieces of data may be collected. The data is stored as a $4 n \times 4 n$ matrix, $Q$, where $Q_{i, j}$ is the conditional probability that a photon exits the system at detector $j$ given that it entered the system at source $i$. Within the array, photons travel in four directions: north, south, east, and west. They change direction by turning some multiple of $\pi / 2$. They do not interact and may be absorbed within a pixel. Photons move according to a two step Markov process. The probabilities with which a photon moves to a neighboring pixel depend upon its previous, as well as present, location. In this two step formulation, the state space consists of locations. The state space may be redefined so that photons move according to a one step Markov process. In the new state space a single state accounts for a photon's location at the previous time step and its present location. There are three different types of these Markov states: incoming, outgoing, and hidden. The probabilities with which photons move from one state to another are referred to as transition probabilities. The transition matrix, $M$, is sparse and may be written as a block matrix. $M$ 's nontrivial subblocks are referred to as $P_{i o}, P_{i h}, P_{h o}$, and $P_{h h}$. $P_{i o}$, for example, contains the probabilities with which photons in incoming states move directly to outgoing states. $P_{\text {ih }}$ contains the probabilities with which photons in incoming states move to hidden states. $P_{h o}$ and $P_{h h}$ are the transition matrices for photons starting in hidden states travelling to outgoing and hidden states, respectively. $P_{i o}$ and $P_{h h}$ are always square matrices. If the Markov states are ordered carefully, all four of these submatrices of $M$ have 
a nice block structure.

The data matrix, $Q$, is $4 n \times 4 n$. $Q_{i, j}$ represents the probability that a photon which enters the system at source $i$ exits the system at detector $j$. Notice that $Q$ provides no time-of-flight information. The forward map is a function of the transition probabilities and equals $Q$. The goal of diffuse tomographers is to invert this map. Given $Q$, we want to recover the transition probabilities. For a given object the transition probabilities give a discretized "image" of the object. In traditional imaging, one recovers a single parameter for each pixel. From this information a visual picture of the object is made. In diffuse tomography, however, one recovers many parameters per pixel. From this information one could make several "pictures" of the object. In both classical and diffuse tomography, fine discretizations of the covering array provide clearer "images" than coarse discretizations.

The clarity of diffuse tomographic "images" will probably never match that of CT and MRI. Linear data analysis is certainly preferable to the nonlinear analysis in the following chapters. Unfortunately, $\mathrm{X}$ rays and supermagnets are expensive and potentially dangerous. Low energy imaging techniques have fewer side effects and are less expensive than high energy methods. Ultrasound, for example, is relatively inexpensive and harmless. MRI, however, would not be cost effective for annual mammograms; PET and CT are not safe for premature infants. It is hoped that optical imaging will become an inexpensive and safe imaging technique; it is the author's hope that diffuse tomographic models will aid researchers in optical imaging. 


\section{Chapter 2}

\section{The Direct Problem}

Because a thorough understanding of the simplest nontrivial system in the plane is the cornerstone of Chapter 3 , a detailed description of the $2 \times 2$ problem follows. Later larger two dimensional systems and as well as $d$ dimensional systems will be discussed.

Consider the setup for the $2 \times 2$ problem as shown in figure 2.1. On each of the eight outer edges there are two devices. One device shoots photons across the outside

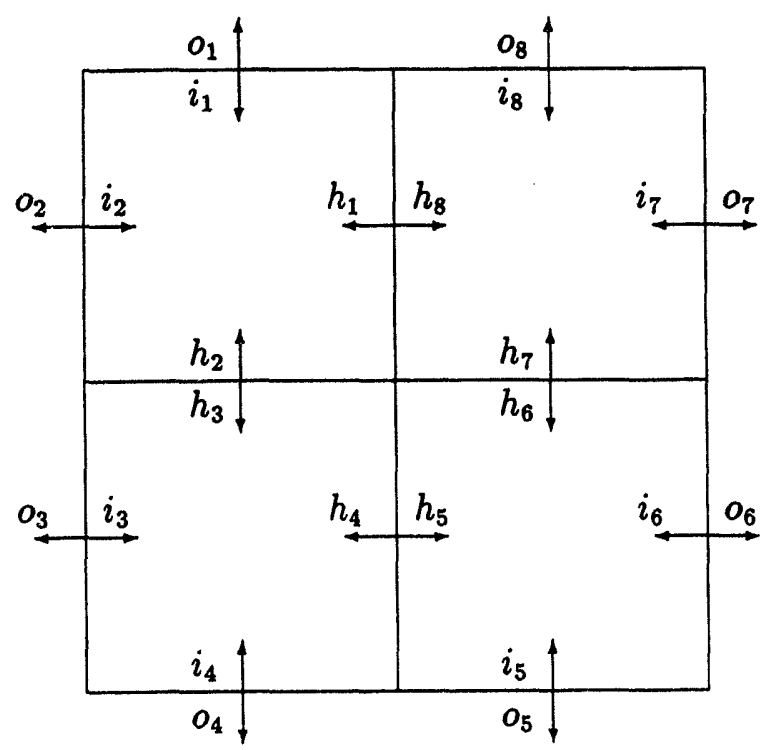

Figure 2.1: Incoming, hidden, and outgoing states are labeled with $i$ 's, $h$ 's, and $o$ 's respectively. 
edge into the neighboring pixel; the other device detects photons as they leave the system. Photons change direction by turning an integral multiple of $\pi / 2$. Photons do not interact. Another property of this model is that a photon may die within a pixel.

Photons travelling according to the above rules are simply moving according to a two step Markov process whose state space consists of locations. When a photon enters pixel $i, j$ from a particular direction it either dies or continues its journey. The probabilities with which the photon moves forward, backwards, left or right are functions of its previous as well as present location. To simplify analysis the state space is redefined in order to make the process a one step Markov process. In the new state space, the previous as well as present location of a photon define its state. Equivalently, the location and direction of travel determine a photon's state. There are one "dead" and 24 "living" states. The "living" states are listed below

$i_{1}, i_{2}, \ldots, i_{8}, h_{1}, h_{2}, \ldots, h_{8}$, and $o_{1}, o_{2}, \ldots, o_{8}$

There are three classes of "living" states: incoming, hidden, and outgoing. See figure figure 2.1. The "dead" state and all of the outgoing states are absorbing states. There are four states by which a photon may enter a given pixel. Once inside the pixel there are five things the photon may do. It may turn right, turn left, reverse directions, continue straight through the pixel, or it may die inside the pixel. The first four transitions are referred to as dynamic transitions. Each of these five events occurs with some transition probability and the sum of the probabilities is identically one. It is sufficient, therefore, to recover only the four dynamic transition probabilities. There are $4 \times 4=16$ dynamic transition probabilities for each of the four pixels, yielding a total of 64 unknowns.

The one step Markov transition matrix, $M$, has a sparse block structure. Ordering the Markov states so that the incoming states precede the hidden states, which precede the outgoing states, gives $M$ the following block structure:

$$
M=\left[\begin{array}{ccc}
0 & P_{i h} & P_{i o} \\
0 & P_{h h} & P_{h o} \\
0 & 0 & I
\end{array}\right]
$$

$M_{i, j}=$ the probability that a photon in state $i$ moves directly to state $j . P_{i o}, P_{i h}$, $P_{h h}$, and $P_{h o}$ are one step transition matrices. They are sparse and their nonzero entries are the dynamic transition probabilities. For example, $P_{i o}[s, t]=$ the probability of a photon 


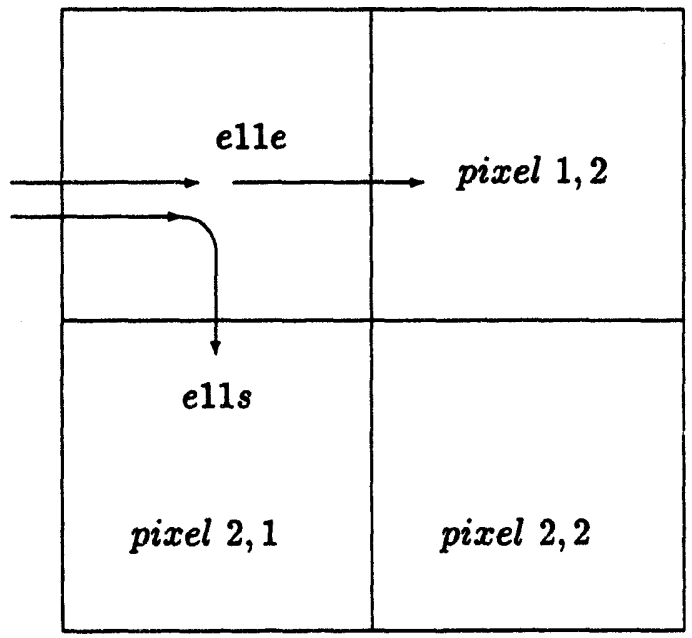

Figure 2.2: The probability that the photon will travel east into pixel 1,1 and continue east into pixel 1,2 is written as e1le. The probability that it will turn right and travel into pixel 2,1 is written as $e 11 s$.

moving from incoming state $s$ directly to outgoing state $t ; P_{i h}[s, t]=$ the probability of a photon moving from incoming state $s$ directly to hidden state $t$. The one step transition matrices for the $2 \times 2$ problem are shown below. Note the sparse $2 \times 2$ block structure of the submatrices for the $2 \times 2$ system and recall the notation as described in figure 2.2 .

$$
P_{i h}=\left[\begin{array}{cccccccc}
0 & 0 & s 11 s & 0 & 0 & 0 & 0 & s 11 e \\
0 & 0 & e 11 s & 0 & 0 & 0 & 0 & e 11 e \\
0 & e 21 n & 0 & 0 & e 21 e & 0 & 0 & 0 \\
0 & n 21 n & 0 & 0 & n 21 e & 0 & 0 & 0 \\
0 & 0 & 0 & n 22 w & 0 & 0 & n 22 n & 0 \\
0 & 0 & 0 & w 22 w & 0 & 0 & w 22 n & 0 \\
w 12 w & 0 & 0 & 0 & 0 & w 12 s & 0 & 0 \\
s 12 w & 0 & 0 & 0 & 0 & s 12 s & 0 & 0
\end{array}\right]
$$




\begin{tabular}{|c|c|c|c|c|c|c|c|c|c|c|c|c|c|c|c|}
\hline \multicolumn{8}{|c|}{$\begin{array}{l}\widehat{N} \\
\stackrel{0}{*}\end{array}$} & \multicolumn{8}{|c|}{ 胥 } \\
\hline & & & $\begin{array}{l}x \\
11 \\
0\end{array}$ & & & & & & & & $\begin{array}{l}5 \\
11\end{array}$ & & & & \\
\hline 0 & 0 & 0 & 0 & 0 & 0 & $\stackrel{\overbrace{}}{\leftrightarrows}$ & $\stackrel{\infty}{5}$ & 芯 & 䒕 & 0 & 0 & 0 & 0 & 0 & 0 \\
\hline 0 & 0 & 0 & 0 & 0 & 0 & $\stackrel{\stackrel{B}{E}}{\varepsilon}$ & $\stackrel{\infty}{\stackrel{\omega}{E}}$ & 0 & 0 & 0 & 0 & 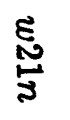 & 鸪 & 0 & 0 \\
\hline 0 & 0 & 0 & 0 & 楚 & 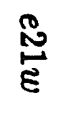 & 0 & 0 & 0 & 0 & 0 & 0 & 0 & 0 & $\underset{\infty}{\vec{t}}$ & $\underset{\infty}{\xi}$ \\
\hline 0 & 0 & 0 & 0 & 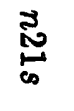 & $\underset{\infty}{\mathbb{E}}$ & 0 & 0 & 0 & 0 & $\begin{array}{l}\text { స్心 } \\
\text { E్ }\end{array}$ & $\begin{array}{l}\text { ్ㅗㄹ } \\
\text { है }\end{array}$ & 0 & 0 & 0 & 0 \\
\hline 0 & 0 & 䄊 & 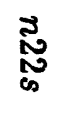 & 0 & 0 & 0 & 0 & 0 & 0 & 0 & 0 & 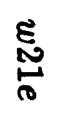 & 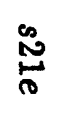 & 0 & 0 \\
\hline 0 & 0 & 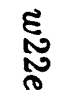 & 胥 & 0 & 0 & 0 & 0 & $\underset{\infty}{\stackrel{0}{*}}$ & $\underset{\infty}{\stackrel{Z}{*}}$ & 0 & 0 & 0 & 0 & 0 & 0 \\
\hline 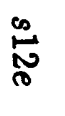 & 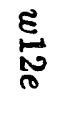 & 0 & 0 & 0 & 0 & 0 & 0 & 0 & 0 & 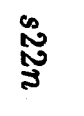 & 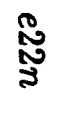 & 0 & 0 & 0 & 0 \\
\hline$\stackrel{\infty}{\widetilde{Z}}$ & 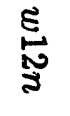 & 0 & 0 & 0 & 0 & 0 & 0 & 0 & 0 & 0 & 0 & 0 & 0 & $\underset{⿱ 乛}{\stackrel{z}{*}}$ & $\underset{\sigma}{\underline{\xi}}$ \\
\hline
\end{tabular}




$$
P_{h_{0}}=\left[\begin{array}{cccccccc}
w 11 n & w 11 w & 0 & 0 & 0 & 0 & 0 & 0 \\
n 11 n & n 11 w & 0 & 0 & 0 & 0 & 0 & 0 \\
0 & 0 & s 21 w & s 21 s & 0 & 0 & 0 & 0 \\
0 & 0 & w 21 w & w 21 s & 0 & 0 & 0 & 0 \\
0 & 0 & 0 & 0 & e 22 s & e 22 e & 0 & 0 \\
0 & 0 & 0 & 0 & s 22 s & s 22 e & 0 & 0 \\
0 & 0 & 0 & 0 & 0 & 0 & n 12 e & n 12 n \\
0 & 0 & 0 & 0 & 0 & 0 & e 12 e & e 12 n
\end{array}\right]
$$

Submatrices for other systems are not always square. For a two dimensional $n \times n$ system, there are $4 n$ incoming and $4 n$ outgoing states and $4 n^{2}-4 n$ hidden states. Hence for a $n \times n$ problem, $P_{i o}$ is $4 n \times 4 n, P_{i h}$ is $4 n \times 4 n(n-1), P_{h h}$ is $4 n(n-1) \times 4 n(n-1)$, and $P_{h o}$ is $4 n(n-1) \times 4 n$. In three dimensions, there are $6 n^{2}$ incoming and outgoing states and $6 n^{3}-6 n^{2}=6 n^{2}(n-1)$ hidden states for a $n \times n \times n$ system. In this case, $P_{i o}$ is a $6 n^{2} \times 6 n^{2}$ matrix, while $P_{i h}$ is $6 n^{2} \times 6 n^{2}(n-1), P_{h h}$ is $6 n^{2}(n-1) \times 6 n^{2}(n-1)$, and $P_{h o}$ is $6 n^{2}(n-1) \times 6 n^{2}$. More generally, in $d$ dimensions an $n \times n \times \ldots \times n$ system is made up of $n^{d}$ $d$-dimensional cubes and has $2 d$ large outer faces. Each of these large outer faces contains $n^{(d-1)}$ faces of individual cubes. Therefore this system has $2 d n^{(d-1)}$ incoming and $2 d n^{(d-1)}$ outgoing states, and $2 d n^{d}-2 d n^{(d-1)}=2 d n^{(d-1)}(n-1)$ hidden states. $P_{i o}$ for this system is $2 d n^{(d-1)} \times 2 d n^{(d-1)}, P_{i h}$ is $2 d n^{(d-1)} \times 2 d n^{(d-1)}(n-1), P_{h h}$ is $2 d n^{(d-1)}(n-1) \times 2 d n^{(d-1)}(n-1)$, and $P_{h o}$ is $2 d n^{(d-1)}(n-1) \times 2 d n^{(d-1)}$.

For $k \in \mathbb{N}$, the $i, j$ entry of the $k^{\text {th }}$ power of $M$ is the probability that a photon starting in state $i$ reaches state $j$ after $k$ Markov steps.

$$
M^{k}=\left[\begin{array}{ccc}
0 & P_{i h} P_{h h}^{(k-1)} & Q^{k} \\
0 & P_{h h}^{k} & \sum_{n=0}^{k-1} P_{h h}^{n} P_{h o} \\
0 & 0 & I
\end{array}\right]
$$

where 


$$
Q^{k}=P_{i 0}+P_{i h}\left(\sum_{n=0}^{k-2} P_{h h}^{n}\right) P_{h o}
$$

$Q_{i, j}^{k}$ is the probability that a photon which entered the system in incoming state $i$ exits the system via outgoing state $j$ during the first $k$ transitions. Because we have no time-of-flight information the data we collect is

$$
Q==_{k \rightarrow \infty}^{l i m} Q^{k}=P_{i o}+P_{i h}\left(\sum_{n=0}^{\infty} P_{h h}^{n}\right) P_{h o}=P_{i o}+P_{i h}\left(I-P_{h h}\right)^{-1} P_{h o}
$$

It is not difficult to show that the sum converges. Although the bulk of the research done to date is on two dimensional models, equation 2.8 holds in any dimension. We say that one solves the forward problem when one calculates $Q$ from $P_{i o}, P_{h h}, P_{h o}$, and $P_{i h}$. Let $f$ denote the forward map given by 2.8 , so $f\left(P_{i o}, P_{i h}, P_{h o}, P_{h h}\right)=Q$. For a $k$-dimensional system, there are $4 k^{2}$ transition probabilities per voxel since a photon may enter a given voxel via any one of $2 k$ states and may exit the voxel via $2 k$ different states. Therefore, $4 k^{2} n^{k}$ of the entries in $P_{i o}, P_{h h}, P_{h o}$, and $P_{i h}$ are nonzero for a $n \times n \times \ldots \times n$ system. Although this thesis concentrates on the algebraic inverse problem, there are physical constraints upon $\operatorname{dom}(f)$ and $\operatorname{Im}(f)$. Let $d_{1}, d_{2}, \ldots, d_{2 k}$ be the preferred directions of travel within the system and let $\alpha$ be a multi-index $\alpha=\left(\alpha_{1}, \alpha_{2}, \ldots, \alpha_{2 k}\right)$ where $\alpha_{i}=1,2, \ldots, n$ for each $i=1,2, \ldots, 2 k$. Then the domain of the forward map lies in the unit cube in $\mathbb{R}^{4 k^{2} n^{k}}$ and satisfies

$$
0 \leq \sum_{j=1}^{4 k^{2} n^{k}} d_{i} \alpha d_{j} \leq 1 \quad \forall \alpha \quad \text { and } \quad i=1,2, \ldots, 2 k
$$

There are similar restrictions upon the range. For a $n \times n \times \ldots \times n$ system $f$ maps the transition probabilities to the $2 k n^{(k-1)} \times 2 k n^{(k-1)}$ matrix $Q \in \operatorname{Im}(f) \subset M a t_{\mathbb{R}}\left(2 k n^{(k-1)}\right)$ and since $Q$ is a transition matrix the following conditions must hold:

$$
0 \leq \sum_{\lambda=1}^{2 k n^{(k-1)}} Q_{i, \lambda} \leq 1 \quad i=1,2, \ldots, 2 k n^{(k-1)}
$$

Let $J a c$ denote the Jacobian. Then if $\operatorname{rank}(\operatorname{Jac}(f(\mathbf{x})))<4 k^{2} n^{k}$ we cannot hope to invert $f$ at the point $\mathbf{x}$. If $\operatorname{rank}(\operatorname{Jac}(f))=r$ at a generic point, then at best we can 
express the transition probabilities in terms of the data and $l$ independent parameters, where $l=4 k^{2} n^{k}-r$. We shall do just this for two dimensional problems in the following chapter. In Chapter 4 a small problem in three dimensions will be studied. 


\section{Chapter 3}

\section{Two Dimensional Problems}

We begin with a study of conditions upon the range of the forward map for problems in the plane. Later we develop an inversion algorithm which respects and takes advantage of these consistency conditions amongst the data.

\subsection{Consistency Conditions}

Consistency conditions amongst the boundary data have the unfortunate effect of reducing the amount of independent data. When working on an inverse problem, we would like to have as much data as possible. At best, we may recover as many parameters as independent data. In the first part of this chapter consistency conditions amongst the boundary data are derived and a few examples are given. In these examples we study boundary data for problems of increasing complexity. It is shown that the number of independent consistency conditions increases faster than the amount of data. Later, the ratio of independent consistency condition's to total data is studied as the complexity increases. However, the coarsest nontrivial array provides a good starting point for this study of consistency conditions.

\subsubsection{Derivation for the $2 \times 2$ problem}

During earlier work on inverting the forward map for the $2 \times 2$ problem, consistency conditions amongst the data were found. Since the theory behind these conditions is the same for $2 \times 2$ arrays as $m \times n$ arrays, the derivation for the consistency conditions in the $2 \times 2$ problem precedes the general derivation. 
In order to recover the probabilities at least as many independent data are required as there are unknowns. Recall the eight detectors positioned around the outer edges of the system. When a photon is shot into the system through an outer edge, the photon either dies somewhere inside the system or is detected as it leaves the system. By collecting data on many photons which enter through the same edge, one may calculate the probability that a photon entering the system through edge $s$ will exit through edge $t$ (here $s, t=1,2, \ldots, 8$ ). The $8 \times 8$ data matrix, $Q$, contains 64 pieces of data.

During early work on this inverse problem the author stumbled upon many zero valued $3 \times 3$ minors of $Q$. In this section, consistency conditions amongst the data are derived. These conditions force the following $4 \times 4$ submatrices of the $8 \times 8$ data matrix to be of rank $\leq 2$.

$$
\begin{gathered}
{\left[\begin{array}{llll}
Q_{1,5} & Q_{1,6} & Q_{1,7} & Q_{1,8} \\
Q_{2,5} & Q_{2,6} & Q_{2,7} & Q_{2,8} \\
Q_{3,5} & Q_{3,6} & Q_{3,7} & Q_{3,8} \\
Q_{4,5} & Q_{4,6} & Q_{4,7} & Q_{4,8}
\end{array}\right],\left[\begin{array}{llll}
Q_{5,1} & Q_{5,2} & Q_{5,3} & Q_{5,4} \\
Q_{6,1} & Q_{6,2} & Q_{6,3} & Q_{6,4} \\
Q_{7,1} & Q_{7,2} & Q_{7,3} & Q_{7,4} \\
Q_{8,1} & Q_{8,2} & Q_{8,3} & Q_{8,4}
\end{array}\right]} \\
{\left[\begin{array}{llll}
Q_{7,3} & Q_{7,4} & Q_{7,5} & Q_{7,6} \\
Q_{8,3} & Q_{8,4} & Q_{8,5} & Q_{8,6} \\
Q_{1,3} & Q_{1,4} & Q_{1,5} & Q_{1,6} \\
Q_{2,3} & Q_{2,4} & Q_{2,5} & Q_{2,6}
\end{array}\right], \text { and }\left[\begin{array}{llll}
Q_{3,7} & Q_{3,8} & Q_{3,1} & Q_{3,2} \\
Q_{4,7} & Q_{4,8} & Q_{4,1} & Q_{4,2} \\
Q_{5,7} & Q_{5,8} & Q_{5,1} & Q_{5,2} \\
Q_{6,7} & Q_{6,8} & Q_{6,1} & Q_{6,2}
\end{array}\right]}
\end{gathered}
$$

By taking advantage of the Markovian nature of the model, we may easily prove that these matrices are rank deficient. Define

$$
\begin{aligned}
p_{i, j}= & \text { probability of going "directly" from } \\
& \text { incoming state } i \text { to hidden state } j \\
s_{i, j}= & \text { probability of starting in hidden state } \\
& i \text { and ever reaching outgoing state } j
\end{aligned}
$$


For the purpose of deriving the rank deficiency of matrix 3.1 a photon is said to travel "directly" if its path from incoming state $i$ to hidden state $j$ includes only one crossing of the thick vertical barrier as shown in figure 3.4. For example, two of the paths $p_{1,8}$ takes into account are shown in figure 3.4. One of the paths which $s_{8,5}$ represents is shown in figure 3.5. Note that $p_{1,8}$ does not include the probability with which a photon travels as shown in figure 3.6.

Referring to figure 2.1 and the definitions given above, some of the $Q_{i, j}$ s may be expressed in terms of the $p_{i, j} \mathrm{~s}$ and $s_{i, j} \mathrm{~s}$

$$
\begin{aligned}
& Q_{1,5}=p_{1,8} s_{8,5}+p_{1,5} s_{5,5} \\
& Q_{1,6}=p_{1,8} s_{8,6}+p_{1,5} s_{5,6} \\
& Q_{1,7}=p_{1,8} s_{8,7}+p_{1,5} s_{5,7} \\
& Q_{1,8}=p_{1,8} s_{8,8}+p_{1,5} s_{5,8}
\end{aligned}
$$

or,

$$
\left[\begin{array}{llll}
Q_{1,5} & Q_{1,6} & Q_{1,7} & Q_{1,8}
\end{array}\right]=\left[\begin{array}{ll}
p_{1,8} & p_{1,5}
\end{array}\right]\left[\begin{array}{llll}
s_{8,5} & s_{8,6} & s_{8,7} & s_{8,8} \\
s_{5,5} & s_{5,6} & s_{5,7} & s_{5,8}
\end{array}\right]
$$

Notation: $Q_{l r}$ denotes the $4 \times 4$ submatrix of the probabilities with which a photon traveis from left to right across the system, starting from sources $1,2,3$ or 4 and ending at detectors $5,6,7$ or 8 . Similarly, $Q_{r l}$ denotes the submatrix of probabilities with which photons travel from right to left across the system. $Q_{t b}$ and $Q_{b t}$ are $4 \times 4$ submatrices representing the probabilities of travel from bottom to top and top to bottom, respectively.

The entire $Q_{l r}$ submatrix can be expressed in the same notation:

$$
\left[\begin{array}{llll}
Q_{1,5} & Q_{1,6} & Q_{1,7} & Q_{1,8} \\
Q_{2,5} & Q_{2,6} & Q_{2,7} & Q_{2,8} \\
Q_{3,5} & Q_{3,6} & Q_{3,7} & Q_{3,8} \\
Q_{4,5} & Q_{4,6} & Q_{4,7} & Q_{4,8}
\end{array}\right]=\left[\begin{array}{ll}
p_{1,8} & p_{1,5} \\
p_{2,8} & p_{2,5} \\
p_{3,8} & p_{3,5} \\
p_{4,8} & p_{4,5}
\end{array}\right]\left[\begin{array}{llll}
s_{8,5} & s_{8,6} & s_{8,7} & s_{8,8} \\
s_{5,5} & s_{5,6} & s_{5,7} & s_{5,8}
\end{array}\right]
$$

Similarly, the $Q_{t b}, Q_{r l}$, and $Q_{b t}$ submatrices may be written as follows: 


$$
\left[\begin{array}{llll}
Q_{7,3} & Q_{7,4} & Q_{7,5} & Q_{7,6} \\
Q_{8,3} & Q_{8,4} & Q_{8,5} & Q_{8,6} \\
Q_{1,3} & Q_{1,4} & Q_{1,5} & Q_{1,6} \\
Q_{2,3} & Q_{2,4} & Q_{2,5} & Q_{2,6}
\end{array}\right]-\left[\begin{array}{ll}
p_{7,3} & p_{7,6} \\
p_{8,3} & p_{8,6} \\
p_{1,3} & p_{1,6} \\
p_{2,3} & p_{2,6}
\end{array}\right]\left[\begin{array}{llll}
s_{3,3} & s_{3,4} & s_{3,5} & s_{3,6} \\
s_{6,3} & s_{6,4} & s_{6,5} & s_{6,6}
\end{array}\right]
$$

$$
\left[\begin{array}{llll}
Q_{5,1} & Q_{5,2} & Q_{5,3} & Q_{3,4} \\
Q_{6,1} & Q_{6,2} & Q_{6,3} & Q_{6,4} \\
Q_{7,1} & Q_{7,2} & Q_{7,3} & Q_{7,4} \\
Q_{8,1} & Q_{8,2} & Q_{8,3} & Q_{8,4}
\end{array}\right]=\left[\begin{array}{ll}
p_{5,4} & p_{5,1} \\
p_{6,4} & p_{6,1} \\
p_{7,4} & p_{7,1} \\
p_{8,4} & p_{8,1}
\end{array}\right]\left[\begin{array}{llll}
s_{4,1} & s_{4,2} & s_{4,3} & s_{4,4} \\
s_{1,1} & s_{1,2} & s_{1,3} & s_{1,4}
\end{array}\right]
$$

$$
\left[\begin{array}{llll}
Q_{3,7} & Q_{3,8} & Q_{3,1} & Q_{3,2} \\
Q_{4,7} & Q_{4,8} & Q_{4,1} & Q_{4,2} \\
Q_{5,7} & Q_{5,8} & Q_{5,1} & Q_{5,2} \\
Q_{6,7} & Q_{6,8} & Q_{6,1} & Q_{6,2}
\end{array}\right]=\left[\begin{array}{ll}
p_{3,2} & p_{3,7} \\
p_{4,2} & p_{4,7} \\
p_{5,2} & p_{5,7} \\
p_{6,2} & p_{6,7}
\end{array}\right]\left[\begin{array}{llll}
s_{2,7} & s_{2,8} & s_{2,1} & s_{2,2} \\
s_{7,7} & s_{7,8} & s_{7,1} & s_{7,2}
\end{array}\right]
$$

Since each of these $4 \times 4$ submatrices is the product of a $4 \times 2$ matrix with a $2 \times 4$ matrix, these $4 \times 4$ submatrices are of rank (at most) 2 .

\subsubsection{Derivation for the $m \times n$ problem}

Moving on to a general description and existence proof of consistency conditions, consider an $m \times n$ problem as shown in figure 3.7. Let $Q_{l r}$ be the submatrix representing the probabilities of photons which enter the system on the left and exit on the right of the thick vertical line. In this case, 


$$
Q_{l r}=\left[\begin{array}{cccc}
Q_{1, m+2 k+1} & Q_{1, m+2 k+2} & \ldots & Q_{1, N} \\
Q_{2, m+2 k+1} & Q_{2, m+2 k+2} & \ldots & Q_{2, N} \\
\vdots & \vdots & & \vdots \\
Q_{m+2 k, m+2 k+1} & Q_{m+2 k, m+2 k+2} & \ldots & Q_{m+2 k, N}
\end{array}\right]
$$

where $N=2(m+n)$.

Claim: rank $\left(Q_{l r}\right) \leq m$.

Proof : For any $Q_{i, j}$ in $Q_{l r}$

$$
Q_{i, j}=\sum_{\alpha=1}^{m} p_{i, \alpha} s_{\alpha, j}, \text { i.e. }
$$

\section{$(3.10) Q_{l r}=$}

$$
\left[\begin{array}{cccc}
p_{1,1} & p_{1,2} & \ldots & p_{1, m} \\
p_{2,1} & p_{2,2} & \ldots & p_{2, m} \\
\vdots & \vdots & & \vdots \\
p_{m+2 k, 1} & p_{m+2 k, 2} & \ldots & p_{m+2 k, m}
\end{array}\right]\left[\begin{array}{cccc}
s_{1, m+2 k+1} & s_{1, m+2 k+2} & \ldots & s_{1, N} \\
s_{2, m+2 k+1} & s_{2, m+2 k+2} & \ldots & s_{2, N} \\
\vdots & \vdots & & \vdots \\
s_{m, m+2 k+1} & s_{m, m+2 k+2} & \ldots & s_{m, N}
\end{array}\right]
$$

Since $Q_{l r}$ is the product of a $(m+2 k) \times m$ matrix with a $m \times(2(n-k)+m)$ matrix the rank of $Q_{t r}$ is at most $m$. The same argument holds for $Q_{r l}, Q_{t b}$, and $Q_{b t}$, (although the ranks of $Q_{t b}$ and $Q_{b t}$ are no greater than $n$ ).

Now consider an even more general left-right transition submatrix. $Q_{l r}$ represents the probabilities of photons which start out on the left of the barrier and exit the system on the right of the barrier shown in figure 3.8.

Without loss of generality assume that $1 \leq l<k<n$. Then 


$$
Q_{l r}=\left[\begin{array}{cccc}
Q_{1, M+1} & Q_{N-l+1, M+2} & \ldots & Q_{1, N} \\
Q_{2, M+1} & Q_{N-l+2, M+2} & \ldots & Q_{2, N} \\
\vdots & \vdots & & \vdots \\
Q_{M, M+1} & Q_{N, M+2} & \ldots & Q_{M, N}
\end{array}\right]
$$

where $N=2(m+n)$ and $M=m+k+l$. For any $Q_{i, j}$ in $Q_{l r}$

$$
Q_{i, j}=\sum_{\alpha=1}^{m+k-l} p_{i, \alpha} s_{\alpha, j}, \text { i.e., }
$$

so in this case $Q_{l r}$ equals

$$
\left[\begin{array}{cccc}
p_{1,1} & p_{1,2} & \ldots & p_{2 m+2 n-l+1, m+k-1} \\
p_{2,1} & p_{2,2} & \ldots & p_{2 m+2 n-l+2, m+k-l} \\
\vdots & \vdots & & \vdots \\
p_{M, 1} & p_{M, 2} & \ldots & p_{M, m+k-l}
\end{array}\right]\left[\begin{array}{cccc}
s_{1, M+1} & s_{1, M+2} & \ldots & s_{1, N} \\
s_{2, M+1} & s_{2, M+2} & \ldots & s_{2, N} \\
\vdots & \vdots & & \vdots \\
s_{m+k-l, M+1} & s_{m+k-l, M+2} & \ldots & s_{m+k-l, N}
\end{array}\right]
$$

$Q_{l r}$ is the product of an $(l+m+k) \times(m+k-l)$ matrix with an $(m+k-l) \times$ $(2 n+m-l-k)$ matrix. Hence, $\operatorname{rank}\left(Q_{l r}\right) \leq(m+k-l)$. The same sort of argument holds for the other rank deficient submatrices. For the barrier in figure 3.8, $\operatorname{rank}\left(Q_{r l}\right) \leq m+k-l$.

\subsubsection{Data subject to Conditions}

For a $n \times n$ system, there are $16 n^{2}$ pieces of data. These data are not all independent, however. Data which are part of some rank deficient submatrix are subject to consistency conditions. In fact, only the data corresponding to nonzero entries of $P_{i o}$ are independent of all consistency conditions. 
It is not difficult to see that the nonzero entries of $P_{\text {io }}$ correspond to "independent" data. Notice that these entries are precisely those representing the probability that a photon may travel directly from an incoming state to an outgoing state. In other words, if $P_{i o}[i, j] \neq 0$ then it is possible for a photon to travel from source $i$ to detector $j$ without ever visiting a hidden state. Such a photon enters only one pixel during its lifetime, and so never has the opportunity to cross any of the barriers which were used to derive the consistency conditions. Hence, $P_{i o}[i, j] \neq 0$ implies $Q_{i, j}$ is free of the consistency conditions derived in section 3.1.2.

Furthermore, only these data are free of the consistency conditions derived in section 3.1.2. Consider any piece of data $Q_{k, l}$ where $P_{i o}[k, l]=0$ and suppose $Q_{k, l}$ is not part of any rank deficient rank $n$ submatrix. Then there exists no right-left, left-right, top-bottom, or bottom-top barrier between source $k$ and detector $l$. Consider the barriers which immediately surround source $k$. See figure 3.9. (There are three such barriers unless source $k$ shoots photons into a corner pixel. In that case there are only two surrounding barriers.) The barriers do not separate source $k$ from detector $l$, so there is some path from $k$ to $l$ which does not cross any of these barriers. Such a path contains no hidden states, which implies that $P_{i o}[k, l] \neq 0$. But $P_{i o}[k, l]=0$, a contradiction. Hence $Q_{k, l}$ is part of some rank deficient, rank $n$ submatrix.

\subsubsection{Examples for square systems}

Before looking for an asymptotic limit to the number of independent consistency conditions as a function of $n$, consider a few more examples. When $n=1$ the array is a single pixel, and there are no consistency conditions analogous to those derived above. For a single pixel there are 16 independent parameters per pixel. For larger arrays, however, there are fewer independent parameters per pixel. See figure 3.10. 


\subsubsection{1 $2 \times 2$ problem}

For the $2 \times 2$ problem, there are four rank deficient submatrices of the data. There is one left-right, one right-left, one top-bottom, and one bottom-top submatrix. Each submatrix is $4 \times 4$ and rank two. Although the submatrices overlap, each yields four independent consistency conditions amongst the 64 pieces of data. The consistency conditions leave at most $64-4 * 4=48$ independent pieces of data. See figure 3.11. In this relatively small case, both Maple and Macsyma are capable of computing the Jacobian of the forward map. At a generic point the rank of the Jacobian is 48 . Since the rank of the forward map is generically 48 , there are at no other consistency conditions.

\subsubsection{2 $4 \times 4$ problem}

As shown in section 3.1.2, there are three rank four $Q_{l r}$ submatrices; three rank four $Q_{r l}$ submatrices; three rank four $Q_{t b}$ submatrices and three rank four $Q_{b t}$ submatrices. The $Q_{l r}$ submatrices all overlap with each other, as do the other sets of rank four submatrices. None of the $Q_{l r}$ submatrices overlap with any of the $Q_{r l}$ submatrices. Similarly, the $Q_{t b}$ and $Q_{b t}$ submatrices are separated.

The $Q_{l r}$ and $Q_{r l}$ submatrices overlap with the $Q_{t b}$ and $Q_{b t}$ submatrices, however. Recall that these submatrices and the entries of $P_{i o}$ cover the data matrix, $Q$. We would like to know how many of the data are independent. The rank four submatrices can easily be used to show that there are at most 160 independent pieces of data (amongst the 256). From the forward map we may recover at most ten independent parameters per pixel, (assuming that it is possible to recover the same number of parameters per pixel). Clearly, the data which occupies the same positions as nonzero entries in $P_{i o}$ are independent. But what of the other data? Consider first the $8 \times 8$ rank four submatrices. Just as in the $2 \times 2$ case, a $4 \times 4$ block from each submatrix may be written off as redundant. See figure 3.12 . This takes full advantage of the fact that the submatrices are of rank four and accounts for all of the data in the submatrix. Consider next the data which are part of one of the $10 \times 6$ rank four submatrices. Most of this data has already been accounted for because it is part of one of the $8 \times 8$ submatrices. Only the first and last rows contain unaccounted for data. Three entries in each of the end rows are assumed known because they lie in neighboring $8 \times 8$ rank deficient submatrix. 
We need know only one more piece of data in order to calculate the two unknown pieces of data in each end row. Analogous reasoning applies to the $6 \times 10$ rank deficient submatrices. Therefore, we may write off as redundant additional data within the $4 \times 4$ subblocks along the diagonal, as shown in fig 3.12 .

\subsubsection{3 $8 \times 8$ problem}

In this case there are seven rank eight submatrices of each stripe: left-right, rightleft, top-bottom, and bottom-top. Once again all of the left-right submatrices are disjoint from the right-left submatrices, but do overlap with the top-bottom and bottom-top submatrices. (Also, the top-bottom submatrices do not intersect any bottom-top submatrices.) And the union of all of the rank deficient, rank eight submatrices and the entries of $P_{\text {io }}$ cover the data matrix. Below it is shown that for $n=8$ we may recover at most nine independent parameters per pixel from the forward map alone.

Once again begin by considering the $16 \times 16$ rank eight submatrices. They contain $8^{*} 8=64$ redundant pieces of data each. Writing off one $8 \times 8$ block per submatrix takes all of these consistency conditions into account. Now all data within these submatrices is assumed to be known. Next, consider the data which is part of an $18 \times 14$ rank eight submatrix. As before, most of the data is already accounted for. Only the first and last rows lack accounted for data. In both the first and last row, seven pieces of data are assumed known, since they are part of some $16 \times 16$ rank eight submatrix. If in both rows one more piece of data is assumed known, then the six remaining pieces of data may be calculated. The same reasoning applies to the first and last columns of the $14 \times 18$ rank eight submatrices. Similar reasoning applies to the end rows and columns of the $20 \times 12$ and $12 \times 20$ rank eight submatrices. Finally, we write off data in the end rows and columns of the $22 \times 10$ and $10 \times 22$ rank eight submatrices. See figure 3.13. 


$\begin{array}{cc} & \text { independent } \\ n & \text { data/pixel } \\ 1 & 16 \\ 2 & 12 \\ 4 & 10 \\ 8 & 9\end{array}$

\subsubsection{For $n$ a power of 2}

For coarse grids, $(n \leq 8)$, the maximum number of independent data per pixel decreases as $n$ increases. In this section the method by which redundant data was found in the examples is generalized. The chart below shows the number of independent data per pixel for $n \times n$ systems.

Further, notice that for $n>2$ the rectangular (not square) rank deficient submatrices account for the increase in the ratio of redundant data to total data. The data rendered redundant by these rectangular submatrices may be chosen inside $n \times n$ blocks along the diagonal. Therefore, the $n \times n$ blocks of along the diagonal are studied below.

Notice that in the examples, the only necessary data in the blocks along the diagonal form an ' $X$ '. All other data within these blocks is redundant. The reason is not too hard to see, even for general $n=2^{k}, k \in \mathbb{N}$. The redundant data belongs to some rectangular rank deficient submatrix. Such submatrices, however, are mostly accounted for by the data in the corresponding square rank $n$ submatrix. There are at least $2 n$ rows (or columns) common to the square and rectangular submatrices. Consider first one of the $(2 n-2) \times(2 n+2)$ submatrices. Only one column protrudes from either side of the corresponding $2 n \times 2 n$ submatrix. For each of these columns, $n-1$ of the data are accounted for because they are part of an overlapping $2 n \times 2 n$ submatrix. Hence, we need only add one piece of data to each column in order to calculated the rest of the column. If we choose to add the piece of data in the corner of the $n \times n$ block along the diagonal, then the $(2 n-2) \times(2 n+2)$ submatrix corresponding to the first square submatrix now has $n$ pieces of data in the protruding columns, and so the rest of the $(2 n-2) \times(2 n+2)$ submatrix can 
be calculated. Furthermore, one of the protruding rows of a neighboring $(2 n+2) \times(2 n-2)$ rank $n$ submatrix now has $n$ pieces of accounted for data. So we may also calculate the $n-2$ remaining pieces of data in that row. By adding one piece of data to one protruding column, we gain $2(n-2)$ pieces of data. Adding one piece of data to each protruding column allows the calculation of the unaccounted for data in both the $(2 n-2) \times(2 n+2)$ and $(2 n+2) \times(2 n-2)$ rank deficient submatrices. Similarly, the judicious addition of one piece of data to each end column of the $(2 n-4) \times(2 n+4)$ rank eight submatrices permits us to calculate the rest of the unaccounted for data in all of the $(2 n-4) \times(2 n+4)$ and $(2 n+4) \times(2 n-4)$ rank deficient submatrices. We may continue this process until reaching the center of the $n \times n$ block along the diagonal. The data in the center of the block are independent entries since they correspond to nonzero entries of $P_{i o}$. Only $2 n$ pieces of data within each of the diagonal $n \times n$ blocks need be known. The other $n^{2}-2 n$ pieces of data are redundant. And among each of the rank $n, 2 n \times 2 n$ submatrices exactly $n^{2}$ pieces of data are redundant. Since the redundant data may be choosen independently, there are at least $4\left(\left(n^{2}-2 n\right)+n^{2}\right)=8 n(n-1)$ pieces of redundant data, leaving at most $8 n(n+1)$ pieces of independent data. The fraction of independent data decreases as the number of pixels increases.

For most imaging methods, quality improves as pixel size decreases. Large pixels yield grainy images. The more pixels used to image an object, the clearer the image. For $n=2^{k}$, the fraction of independent data approaches $1 / 2$ as $k$ approaches infinity. For $n \geq 16$, the forward map generates at most eight independent data per pixel. Additional information about the system is needed in order to recover diagnostically relvant information from the data. Some a priori knowledge of photon transport is needed to close the system of governing equations derived in the next section. 


\subsection{Recursive Inversion Algorithm}

Although it is not possible to invert the direct map because of the consistency conditions amongst the data, it is possible to recover as much information as there are independent data. For an $n \times n$ system the forward map takes $16 n^{2}$ transition probabilities and maps them to the $4 n \times 4 n$ matrix $Q$. The domain of the forward map lies in the unit cube in $\mathbb{R}^{16 n^{2}}$. The domain is defined by the equations

$$
\begin{aligned}
e i j e+e i j w+e i j n+e i j s & \leq 1 \\
w i j e+w i j w+w i j n+w i j s & \leq 1 \\
n i j e+n i j w+n i j n+n i j s & \leq 1 \\
s i j e+s i j w+s i j n+s i j s & \leq 1
\end{aligned}
$$

for $i, j=1,2, \ldots, n$. Because $Q$ is a transition matrix acceptable solutions lie in $\mathbb{R}^{16 n^{2}}$ and satisfy

$$
0 \leq \sum_{\lambda=1}^{4 n} Q[i, \lambda] \leq 1 \quad i=1,2, \ldots, 4 n
$$

Since the rank of the forward map is less than $16 n^{2}$ we cannot hope to invert it. If the rank of its Jacobian is generically $r$, then at most we can recover $r$ pieces of information. Although we cannot explicitly solve for transition probabilities in terms of the data, we can express them in terms of the data and $k$ independent parameters, where $k=16 n^{2}-r$. In this section a recursive algorithm for finding the $k$ parameter family of solutions is detailed.

\subsubsection{Base Case $(2 \times 2$ problem)}

\subsubsection{Solving the Governing Equations}

By making several nonlinear changes of variables, we may remove the nonlinearities from 2.8, (or "move" them to the changes of variables). First define, (assuming that $P_{h o}$ is invertible),

$$
A=P_{h o}^{-1}
$$

Equation 2.8 may be rewritten as 


$$
\left(Q-P_{i 0}\right) A\left(I-P_{h h}\right)-P_{i h}=\Theta
$$

where $\theta$ is a matrix of zeros. $\theta$ will denote a vector of zeros. A few more changes of variables are required to make 3.16 linear:

$$
\begin{aligned}
W & =A P_{h h} \\
X & =P_{i o} A \\
Y & =P_{i o} W-P_{i h}
\end{aligned}
$$

We can recover $P_{h h}, P_{i o}$, and $P_{i h}$ in terms of $A$ if we know $W, X$, and $Y$. Under these substitutions, the matrix equation 3.16 becomes

$$
Q(A-W)-(X-Y)=\Theta
$$

Recall that $Q$ is the data, so 3.18 is linear in the unknown matrices $A, W, X$, and $Y$. Furthermore, the new matrices have special block structures. $A$ has the same diagonal block structure as $P_{h o} . X$ is also block diagonal. Finally, $W$ and $Y$ have the same off diagonal block structure as $P_{h h}$ and $P_{i h}$. The variables for each system/column of equations contains three each of the $A_{i, j} s, W_{i, j} s, X_{i, j} s$, and $Y_{i, j} s$. The $W_{i, j} s, X_{i, j} s$, and $Y_{i, j} s$ are functions of $A_{i, j} s$ which correspond to other columns. Although the variables differ from column to column (exactly 64 variables total-no repeats between columns), the columns are only artificially decoupled. 
(3.19)

$A-W=$

$$
\left[\begin{array}{cccccccc}
A_{1,1} & A_{1,2} & -W_{1,3} & 0 & 0 & 0 & 0 & -W_{1,8} \\
A_{2,1} & A_{2,2} & -W_{2,3} & 0 & 0 & 0 & 0 & -W_{2,8} \\
0 & -W_{3,2} & A_{3,3} & A_{3,4} & -W_{3,3} & 0 & 0 & 0 \\
0 & -W_{4,2} & A_{4,3} & A_{4,4} & -W_{4,3} & 0 & 0 & 0 \\
0 & 0 & 0 & -W_{5,4} & A_{5,3} & A_{8,0} & -W_{5,7} & 0 \\
0 & 0 & 0 & -W_{6,4} & A_{6,5} & A_{6,6} & -W_{8,7} & 0 \\
-W_{7,1} & 0 & 0 & 0 & 0 & -W_{7,6} & A_{7,7} & A_{7,8} \\
-W_{8,1} & 0 & 0 & 0 & 0 & -W_{8,6} & A_{8,7} & A_{8,8}
\end{array}\right]
$$

$$
X-Y=\left[\begin{array}{cccccccc}
X_{1,1} & X_{1,2} & -Y_{1,3} & 0 & 0 & 0 & 0 & -Y_{1,8} \\
X_{2,1} & X_{2,2} & -Y_{2,3} & 0 & 0 & 0 & 0 & -Y_{2,8} \\
0 & -Y_{3,2} & X_{3,3} & X_{3,4} & -Y_{3,5} & 0 & 0 & 0 \\
0 & -Y_{4,2} & X_{4,3} & X_{4,4} & -Y_{4,5} & 0 & 0 & 0 \\
0 & 0 & 0 & -Y_{5,4} & X_{3,5} & X_{5,6} & -Y_{5,7} & 0 \\
0 & 0 & 0 & -Y_{6,4} & X_{6,3} & X_{6,6} & -Y_{6,7} & 0 \\
-Y_{7,1} & 0 & 0 & 0 & 0 & -Y_{7,6} & X_{7,7} & X_{7,8} \\
-Y_{8,1} & 0 & 0 & 0 & 0 & -Y_{8,6} & X_{8,7} & X_{8,8}
\end{array}\right]
$$

Now the equations in column six of 3.18 are linear in the variables

$$
\left\{A_{5,6}, A_{6,6}, W_{7,6}, W_{8,6}, X_{5,6}, X_{6,6}, Y_{7,6}, Y_{8,6}\right\}
$$

and can be written as a homogeneous matrix equation: 


$$
\left[\begin{array}{llllllll}
Q_{1,8} & Q_{1,6} & Q_{1,7} & Q_{1,8} & 0 & 0 & 0 & 0 \\
Q_{2,8} & Q_{2,0} & Q_{2,7} & Q_{2,8} & 0 & 0 & 0 & 0 \\
Q_{3,5} & Q_{3,6} & Q_{3,7} & Q_{3,8} & 0 & 0 & 0 & 0 \\
Q_{4,5} & Q_{4,8} & Q_{4,7} & Q_{4,8} & 0 & 0 & 0 & 0 \\
Q_{8,5} & Q_{8,6} & Q_{8,7} & Q_{8,8} & 1 & 0 & 0 & 0 \\
Q_{8,5} & Q_{8,6} & Q_{8,7} & Q_{8,8} & 0 & 1 & 0 & 0 \\
Q_{7,5} & Q_{7,6} & Q_{7,7} & Q_{7,8} & 0 & 0 & -1 & 0 \\
Q_{8,5} & Q_{8,6} & Q_{8,7} & Q_{8,8} & 0 & 0 & 0 & -1
\end{array}\right]\left[\begin{array}{c}
A_{8,0} \\
A_{8,6} \\
-W_{7,6} \\
-W_{8,6} \\
X_{8,6} \\
X_{8,6} \\
Y_{7,8} \\
Y_{8,6}
\end{array}\right]=\theta
$$

We can do the same for the other columns in 3.18. To each column in 3.18 there corresponds a system of eight linear equations in the variables which appear in the corresponding columns of 3.19 and 3.20. Note that as far as their zero structures are concerned, the columns of 3.19 and 3.20 come in pairs. The roles of the $A_{i, j} s$ and $W_{i, j} s$ are reversed in the first and eighth columns of 3.19 as are the roles of the $X_{i, j} s$ and $Y_{i, j} s$ in the first and eighth columns of 3.20. Hence, we must solve the "same" matrix equation for the first and eighth columns of 3.18. Similarly, the linear systems corresponding to the second and third columns of 3.18 are given by a single matrix equation; the fourth and fifth columns by a third matrix equation; and the sixth and seventh columns by a fourth matrix equation. We are left with four sets of homogeneous linear equations, i.e., four $8 \times 8$ matrices which satisfy the homogeneous equation $\hat{Q} x=\theta$. Since the trivial solution would not be interesting enough to write about we may safely assume that there must be other solutions. This is indeed the case since the upper left $4 \times 4$ submatrix found in equation 3.21 ,

$$
\left[\begin{array}{llll}
Q_{1,5} & Q_{1,6} & Q_{1,7} & Q_{1,8} \\
Q_{2,5} & Q_{2,6} & Q_{2,7} & Q_{2,8} \\
Q_{3,5} & Q_{3,6} & Q_{3,7} & Q_{3,8} \\
Q_{4,5} & Q_{4,6} & Q_{4,7} & Q_{4,8}
\end{array}\right]
$$

representing travel from left to right across the system is rank deficient. As noted in section 3.1 the submatrix above is of rank two or less. So we may solve 3.21 for at most six of the eight unknowns in terms of the other two. 
The variables for each system of equations contains two each of the $A_{i, j} s, W_{i, j} s$, $X_{i, j} s$, and $Y_{i, j} s$. Do not forget, however, that the $W_{i, j} s, X_{i, j} s$, and $Y_{i, j} s$ are functions of $A_{i, j} \&$ which correspond to other columns. Although the variables differ from column to column (exactly 64 variables total-no repeats between columns), the columns are only artificially decoupled. Recall that only six equations per column of 2.8 are independent. Since the $W_{i, j} s, X_{i, j} s$, and $Y_{i, j} s$ are already functions of $A_{i, j} s$, it seems natural to solve for them in terms of the $A_{i, j} s$. Following this procedure for all eight columns reduces the number of unknowns from 64 to 16.

To solve 3.21 for the $W_{i, j} s, X_{i, j} s$, and $Y_{i, j} s$ in terms of the $A_{i, j} s$, we need only solve:

$$
\left[\begin{array}{llllll}
Q_{3,7} & Q_{3,8} & 0 & 0 & 0 & 0 \\
Q_{4,7} & Q_{4,8} & 0 & 0 & 0 & 0 \\
Q_{8,7} & Q_{5,8} & -1 & 0 & 0 & 0 \\
Q_{6,7} & Q_{6,8} & 0 & -1 & 0 & 0 \\
Q_{7,7} & Q_{7,8} & 0 & 0 & 1 & 0 \\
Q_{8,7} & Q_{8,8} & 0 & 0 & 0 & 1
\end{array}\right]\left[\begin{array}{l}
W_{7,6} \\
W_{8,6} \\
X_{5,6} \\
X_{6,6} \\
Y_{7,6} \\
Y_{8,6}
\end{array}\right]=\left[\begin{array}{ll}
Q_{3,5} & Q_{3,6} \\
Q_{4,5} & Q_{4,6} \\
Q_{8,5} & Q_{5,6} \\
Q_{6,5} & Q_{6,6} \\
Q_{7,5} & Q_{7,6} \\
Q_{8,5} & Q_{8,6}
\end{array}\right]\left[\begin{array}{l}
A_{5,6} \\
A_{6,6}
\end{array}\right]
$$

For the sake of simplicity the first two rows of the matrix equation 3.21 were omitted and the equation was rewritten with the unknown $W_{i, j} s, X_{i, j} s$, and $Y_{i, j} s$ on the lefthand side. The determinant of the lefthand matrix in 3.23 is $d Q_{[3,4],(7,8]} .3 .23$ has. a unique solution if and only if $d Q_{[3,4],[7,8]} \neq 0$. A similar requirement holds for each of the other columns of 3.20. In order to solve each column of equations for the $W_{i, j} s, X_{i, j} s$, and $Y_{i, j} s$ in terms of the $A_{i, j} s$ it is sufficient that the following minors be nonzero.

$$
d Q_{[3,4],[7,8]}, \quad d Q_{[7,8],[3,4]}, \quad d Q_{[5,6],[1,2]}, \quad d Q_{[1,2],\{5,0]}
$$

If the data satisfy these requirements then we can solve the 48 independent equations in 64 variables linearly for the nonzero entries in $W, X$, and $Y$ in terms of the 16 variables in $A=P_{h o}^{-1}$. Unfortunately, that exhausts the supply of equations for the original model. Example solutions from each of the four one step transition submatrices are shown below: 


$$
w 11 w=-\frac{A_{1,2}}{d A_{[1,2],(1,2]}}
$$

where $w 11 w$ is an entry in $P_{h o}$. One of $P_{h h}$ 's nonzero entries is

$$
\begin{aligned}
& w 21 e=\left(\mathrm{dQ}_{[1,2],[3,8]} A_{5,8} A_{3,3}+\mathrm{dQ}_{[1,2],[3,0]} A_{6,8} A_{3,3}+\right. \\
& \left.\mathrm{dQ}_{[1,2],(4,5]} A_{5,8} A_{4,3}+\mathrm{dQ}_{(1,2],[4,0]} A_{6,5} A_{4,3}\right) / \\
& \mathrm{dQ}_{[1,2],(3,4)} d A_{[3,4),[3,4]}
\end{aligned}
$$

and

$$
\begin{aligned}
& n 21 s=\frac{\mathrm{dQ}_{[1,2,4],[4,5,0]} A_{3,3} A_{4,4}}{\mathrm{dQ}_{[1,2],(5,0]} d A_{[3,4],(3,4]}}-\frac{\mathrm{dQ}_{[4,5,0],[1,2,4]} A_{4,3} A_{3,4}}{\mathrm{~d} Q_{[5,0],(1,2]} d A_{[3,4),(3,4]}}+
\end{aligned}
$$

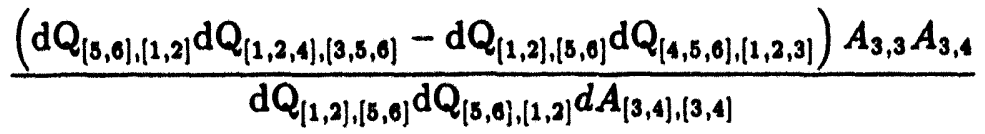

is an entry of $P_{\text {io }}$. Finally, one of the nonzero entries of $P_{\text {in }}$ is

$$
\begin{aligned}
& s 12 s=\left(-\mathrm{dQ}_{[5,0],[1,2]}\left(\mathrm{dQ}_{[1,2],[5,8]} \mathrm{d} \mathrm{Q}_{[1,2,8],(6,7,8]}-\mathrm{dQ}_{[1,2],[6,7]} \mathrm{d} \mathrm{Q}_{[1,2,8],[5,0,8]}\right)\right. \\
& \left.-\mathrm{dQ}_{[1,2],[6,8]} \mathrm{dQ} \mathrm{Q}_{[1,2],[5,0]} \mathrm{dQ} \mathrm{Q}_{[5,0,8],(1,2,7]}\right) A_{6,8} A_{7,8} A_{8,7} \\
& +\left(d Q_{[5,6],[1,2]}\left(d Q_{[1,2],[6,8]} d Q_{[1,2,8],[5,0,7]}+d Q_{[1,2],[5,6]} d Q_{[1,2,8],[6,7,8]}\right)\right. \\
& \left.-\mathrm{dQ}_{[1,2],(6,7]} \mathrm{dQ} \mathrm{Q1,2],(5,8]} \mathrm{d} \mathrm{Q}_{[5,6,8],[1,2,8]}\right) A_{7,7} A_{6,6} A_{8,8} \\
& -\left(-d Q_{[5,8],[1,2]}\left(d Q_{[1,2],[5,0]} d Q_{[1,2,8],[5,7,8]}-d Q_{[1,2],[5,7]} d Q_{[1,2,8],[5,6,8]}\right)\right. \\
& \left.-\mathrm{dQ}_{[1,2],(5,8]} \mathrm{dQ} Q_{[1,2],(3,0]} \mathrm{dQ} Q_{[3,0,8],(1,2,7]}\right) A_{5,6} A_{8,7} A_{7,8} \\
& +\left(d Q_{[5,6],[1,2]}\left(d Q_{[1,2],[5,6]} d Q_{[1,2,8],[5,7,8]}+d Q_{[1,2],[5,8]} d Q_{[1,2,8],[5,6,7]}\right)\right. \\
& \left.-\mathrm{dQ}_{[1,2],(5,7]} \mathrm{d} Q_{[1,2],(5,0)} \mathrm{d} Q_{[5,6,8],(1,2,8]}\right) A_{5,6} A_{8,8} A_{7,7} \\
& +A_{8,7} A_{8,8}\left(\mathrm{dQ}_{[1,2],[6,8]} A_{6,6}+\mathrm{dQ}_{[1,2],[5,8]} A_{5,6}\right) \\
& \left(-d Q_{[5,6,8],[1,2,8]} d Q_{[1,2],[5,8]}+d Q_{[5,0],[1,2]} d Q_{[1,2,8],(5,6,8]}\right) \\
& +A_{7,8} A_{7,7}\left(\mathrm{dQ}_{[1,2],[0,7]} A_{6,8}+\mathrm{dQ}_{[1,2],[5,7]} A_{5,6}\right) \\
& \left.\left(d Q_{[5,6],\{1,2]} d Q_{[1,2,8],[5,6,7]}-d Q_{[1,2],[5,6]} d Q_{[5,6,8],(1,2,7]}\right)\right) / \\
& d A_{[7,8],[7,8]} \mathrm{d} \mathrm{Q}_{[1,2],(7,8]} \mathrm{d} \mathrm{Q}_{[1,2],(5,6)} \mathrm{d} \mathrm{Q}_{[5,0],(1,2]}
\end{aligned}
$$

Solutions for variables from a transition submatrix are all of the same form. For example, all of the transition probabilities in $P_{h o}$ are equal to an entry of $A$ divided by a $2 \times 2$ minor of $A$. 


\subsubsection{Grabmannians and the Graßmann-Plücker Embedding}

Since equation 3.23 is a linear system of six equations in eight unknowns, it is not surprising that Grabmannians and the Grabmann-Plücker embedding come into play. Grabmannians and the identities which embed them in projective space will be used in the following section to simplify solutions for incoming-hidden transition probabilities like that shown in 3.28 .

3.2.1.2.1 Grafmannians Given integers $k$ and $n$, where $k<n, G(k, n)$ is defined as the set of all $k$-dimensional linear spaces in $\mathbb{C}^{n}$. Let $\Lambda$ be an element of $G(k, n)$. Then there exists a set of $k 1 \times n$ spanning vectors of $\Lambda$. Represent $\Lambda$ as a $k \times n$ matrix whose rows are these spanning vectors. Because the choice of spanning vectors is not unique, a family of matrices represent $\Lambda$. Given any $g$ in $G L(k)$, define $\Lambda^{\prime}=g \Lambda$. In the following sections both the point $\Lambda \in G(k, n)$ and a $k \times n$ matrix representing $\Lambda$ will be denoted by $\Lambda$. Hopefully, context will make the author's meaning clear. The rows of $\Lambda^{\prime}$ sr an the same space as the rows of $\Lambda$, so we identify $\Lambda^{\prime}$ and $\Lambda$.

Under these identifications, it is easy to construct a bijection between a dense, open subset of $G(k, n)$ and $\mathbb{C}^{k(n-k)}$. Let $O$ be the set of all points in $G(k, n)$ which may be represented by a $k \times n$ matrix whose first $k$ columns are independent. $O$ is a dense open set in $G(k, n)$. Given any representation for $\Lambda$ in $O$, we can easily find the matrix representation $\bar{\Lambda}$ for $\Lambda$ such that the first $k$ columns of $\bar{\Lambda}$ are the identity matrix. (Simply take $g^{-1}$ to be the first $k$ columns of $\Lambda$. The rows of $\Lambda$ are independent so $g^{-1}$ is invertible. Define $\bar{\Lambda}=g \Lambda$.) Only the entries of the rightmost $k \times(n-k)$ submatrix of $\bar{\Lambda}$ are unconstrained.

More generally, let $I=\left(i_{1}, i_{2}, i_{3}, \ldots, i_{k}\right)$ index $k$ independent columns of the original representation for $\Lambda$. Then given any $\Lambda$, we can define the map $\phi_{I}$ such that $\phi_{I}(\Lambda)$ satisfies the following: column $i_{j}$ of $\phi_{I}(\Lambda)=e_{j}$, where $i_{j}$ is the $j_{t h}$ index in $I$ and $e_{j}$ is the $j_{\text {th }}$ canonical vector. In order to define $\phi_{I}$, first set $g^{-1}$ equal to the matrix with columns $i_{1}, i_{2}, i_{3}, \ldots, i_{k}$ of $\Lambda$. Generically, $g^{-1}$ is of rank $k$ so $g$ exists. Then $\tilde{\Lambda}=g \Lambda$ is has column $i_{j}$ equal to $e_{j}$. The inverse of $\phi_{I}$ is always uniquely defined. 
Let $I^{\prime}$ denote another set of linearly independent columns. Define $\Lambda_{I}=\phi_{I}(\Lambda)$ and $\Lambda_{I^{\prime}}=\phi_{I^{\prime}}(\Lambda)$. Then $\Lambda_{I}$ and $\Lambda_{I^{\prime}}$ satisfy $\Lambda_{I}=g \Lambda$ and $\Lambda_{I^{\prime}}=g^{\prime} \Lambda$ for some $g, g^{\prime}$ in $G L(k)$. Then $\Lambda_{l^{\prime}}=h \Lambda_{I}$ for some matrix $h$ in $G L(k)$. A moment's reflection reveals that $h$ must be the inverse of the matrix composed of the columns of $\Lambda_{I}$ which are indexed by $I^{\prime}$. Note that the entries of $h$ are analytic functions of the entries of $\Lambda_{l}$, so $G(l, n)$ has the structure of a complex manifold.

3.2.1.2.2 The Plücker Embedding Any Graßmannian, $G(k, n)$, may be embedded into $\left.\mathbb{P}^{(}\right)-1 . \mathbb{P}^{N}$ is $N$ dimensional projective space over the complex numbers; we can think of $\mathbb{P}^{N}$ as an $N$ dimensional sphere lying in $N+1$ dimensional space with antipodal points identified. A point, $P$, in $\mathbb{P}^{N}$ may denoted by $\left(p_{0}, p_{1}, p_{2}, \ldots, p_{N}\right)$. This point is identified with all other points $\alpha \times\left(p_{0}, p_{1}, p_{2}, \ldots, p_{N}\right)$ for any nonzero scalar $\alpha$.

In the simplest case, $G(1, n)$, any point of the Grabmannian is represented by a single row vector. Since we may multiply each element of the Graßmannian by a nonzero scalar, we may canonically identify $\mathbb{P}^{n-1}$ and $G(1, n)$. Every element, $\Lambda$, of a $G(k, n)$ defines a $k$ dimensional linear space in $\mathbb{C}^{n}$. The Plücker coordinates of a Graßmannian $\Lambda$ are by definition the determinants of all $k \times k$ minors of any representation $\Lambda$ of an element in $G(k, n)$. The dual space corresponding to $\Lambda$ is $\Lambda^{\perp}$, the $(n-k)$ dimensional linear space in $\mathbb{C}^{n}$ orthogonal to $\Lambda$. There is a $1-1$ correspondence between the Plücker coordinates of $\Lambda$ and $\Lambda^{\perp}$. Since the set of $(n-1)$ dimensional spaces in $\mathbb{C}^{n}$ is isomorphic to the space of one dimensional spaces, we can identify any $\Lambda$ in $G(n-1, n)$ with its dual, $\Lambda^{\perp}$. Hence $G(n-1, n)$ and $\mathbb{P}^{n-1}$ are identified.

When $1<k<(n-1)$ more complicated relations are required to embed $G(k, n)$ in some projective space. It is easy to check that Plücker coordinates are projectively invariant under representation of $\Lambda$. Let $\Lambda$ and $\Lambda^{\prime}$ be equivalent representations for the same element of $G(k, n)$. Then $\Lambda=g \Lambda^{\prime}$ for some $g$ in $G L(k)$. Let $I$ be any index of $k$ columns. The submatrix taken from the $I$ columns of $\Lambda$ equals $g$ times the submatrix taken from the $I$ columns of $\Lambda^{\prime}$. By the rules of determinants, $|A B|=|A||B|$, and so the determinant of the $I_{t h}$ minor of $\Lambda$ equals the determinant of $g$ times the determinant of the $I_{t h}$ minor of $\Lambda^{\prime}$. This holds for all $I$, and so if the $\left(\begin{array}{l}n \\ k\end{array}\right)$-tuple $P$ are the Plücker coordinates of $\Lambda$ then $|g| q$ are the Plücker coordinates of $\Lambda^{\prime}$. Since there are $\left(\begin{array}{l}n \\ k\end{array}\right) \quad k \times k$ minors of a $k \times n$ matrix, the Plücker map takes $G(k, n)$ into $\mathbb{P}^{\left({ }^{n}\right.}\left(\begin{array}{l}n \\ )\end{array}\right)$. (Note that the Plücker map is not onto.) 
We can check that for $1<k<n,\left(\begin{array}{l}n \\ n\end{array}\right)-1 \geq k(n-k)$. In order for the Plücker map to be an embedding, there must be some (i.e., $\left(\left(\begin{array}{l}n \\ n\end{array}\right)-1-k(n-k)\right)$ ) independent relations amongst the Plücker coordinates of a point $\Lambda$ in $G(k, n)$. For $G(2, n)$ these are the Plücker relations. For general $G(k, n)$ they are called Grabmann relations. In either case the relations are quadratic in the Plücker coordinates for $\Lambda$. The Grabmann relations are easily derived.

3.2.1.2.3 Derivation of Graßmann-Plücker Relations Let $\Lambda$ be any rectangular matrix with $k$ rows and $n$ columns where $k<n-1$ and $\Lambda=(a)_{i j}$. Let $I=$ $\left(i_{1}, i_{2}, i_{3}, \ldots, i_{(k-1)}\right)$ index $(k-1)$ distinct columns of $\Lambda$. Let $J=\left(j_{1}, j_{2}, j_{3}, \ldots, j_{(k+1)}\right)$ index $(k+1)$ distinct columns of $\Lambda$. Consider the sum,

$$
\begin{array}{r}
\sum_{\lambda=1}^{k+1}(-1)^{\lambda+1}\left|\begin{array}{ccccc}
a_{1, i_{1}} & a_{1, i_{2}} & \ldots & a_{1, i_{h-1}} & a_{1, j_{\lambda}} \\
\vdots & & & \vdots & \\
a_{h, i_{1}} & \ldots & \ldots & a_{h, i_{h-1}} & a_{k, j_{\lambda}}
\end{array}\right| \\
\left|\begin{array}{cccccc}
a_{1, j_{1}} & \ldots & a_{1, j_{\lambda-1}} & a_{1, j_{\lambda+1}} & \ldots & a_{1, j_{k+1}} \\
\vdots & & \vdots & \vdots & & \\
a_{k, j_{1}} & \ldots & a_{k, j_{\lambda-1}} & a_{k, j_{\lambda+1}} & \ldots & a_{k, j_{k+1}}
\end{array}\right|
\end{array}
$$

To simplify 3.29, expand the first determinant along the last column as shown below

$$
\left|\begin{array}{ccccc}
a_{1, i_{1}} & a_{1, i_{2}} & \cdots & a_{1, i_{k-2}} & a_{1, j_{\lambda}} \\
\vdots & & & & \vdots \\
a_{k, i_{1}} & & & a_{k, i_{h-1}} & a_{h, j_{\lambda}}
\end{array}\right|=\sum_{\mu=1}^{k} a_{\mu, j_{\lambda}} C F_{\mu}
$$

where $C F_{\mu}$ is the cofactor of the matrix on the left-hand side of 3.30 about the $(\mu, k)_{t h}$ entry. Then 3.29 becomes 


$$
\begin{aligned}
& \sum_{\lambda=1}^{h+1}(-1)^{\lambda+1} \sum_{\mu=1}^{h} a_{\mu, j_{\lambda}} C F_{\mu}\left|\begin{array}{cccccc}
a_{1, j_{1}} & \cdots & a_{1, j_{\lambda-1}} & a_{1, j_{\lambda+1}} & \cdots & a_{1, j_{h+1}} \\
\vdots & & \vdots & \vdots & & \vdots \\
a_{h, j_{1}} & \cdots & a_{k, j_{\lambda-1}} & a_{h, j_{\lambda+1}} & \cdots & a_{k, j_{h+1}}
\end{array}\right| \\
& =\sum_{\mu=1}^{n} C F_{\mu} \sum_{\lambda=1}^{h+1}\left|\begin{array}{ccccccc}
0 & \ldots & 0 & a_{\mu, j_{\lambda}} & 0 & \ldots & 0 \\
a_{1, j_{2}} & \ldots & a_{1, j_{\lambda-1}} & a_{1, j_{\lambda}} & a_{1, j_{\lambda+1}} & \ldots & a_{1, j_{h+1}} \\
\vdots & & \vdots & \vdots & & & \vdots \\
a_{h, j_{2}} & \ldots & a_{h, j_{\lambda-1}} & a_{k, j_{\lambda}} & \ldots & \ldots & a_{h, j_{h+1}}
\end{array}\right| \\
& =\sum_{\mu=1}^{k} C F_{\mu}\left|\begin{array}{cccc}
a_{\mu, j_{1}} & a_{\mu, 2} & \ldots & a_{\mu, j_{h+1}} \\
a_{1, j_{1}} & a_{1, j_{2}} & \ldots & a_{1, j_{h+1}} \\
\vdots & \vdots & & \vdots \\
a_{h, j_{1}} & a_{k, j_{2}} & \ldots & a_{k, j_{h+1}}
\end{array}\right| \\
& =\sum_{\mu=1}^{k} C F_{\mu} \cdot 0 \\
& =0
\end{aligned}
$$

Denote by $\pi_{I}$ the determinant of the minor whose columns are indexed by the multi-index $I$. Then

$$
\sum_{\lambda=1}^{k+1} \pi_{\left(i_{1}, i_{2}, \ldots, i_{h-1}, j_{\lambda}\right)} \pi_{\left(j_{1}, j_{2}, \ldots, j_{\lambda-1}, j_{\lambda+1}, \ldots, j_{k+1}\right)}=0
$$

Equation 3.32 defines the Graßmann relations. In the following paragraphs a few simple examples are given.

3.2.1.2.3.1 Examples of Graßmann-Plücker Relations - G(2,4) First we consider $G(2,4)$. Since $G(2,4)$ is isomorphic to a dense, open subset of $\mathbb{C}^{4}$, and the Plücker map takes $G(2,4)$ into $\mathbb{P}^{5}$, only one nontrivial Graßmann relation is required to embed $G(2,4)$ into $\mathbb{P}^{5}$. Consider the representation for $\Lambda \in G(2,4)$

$$
\Lambda=\left[\begin{array}{cccc}
A_{1,1} & A_{1,2} & A_{1,3} & A_{1,4} \\
A_{2,1} & A_{2,2} & A_{2,3} & A_{2,4}
\end{array}\right]
$$

and consider 


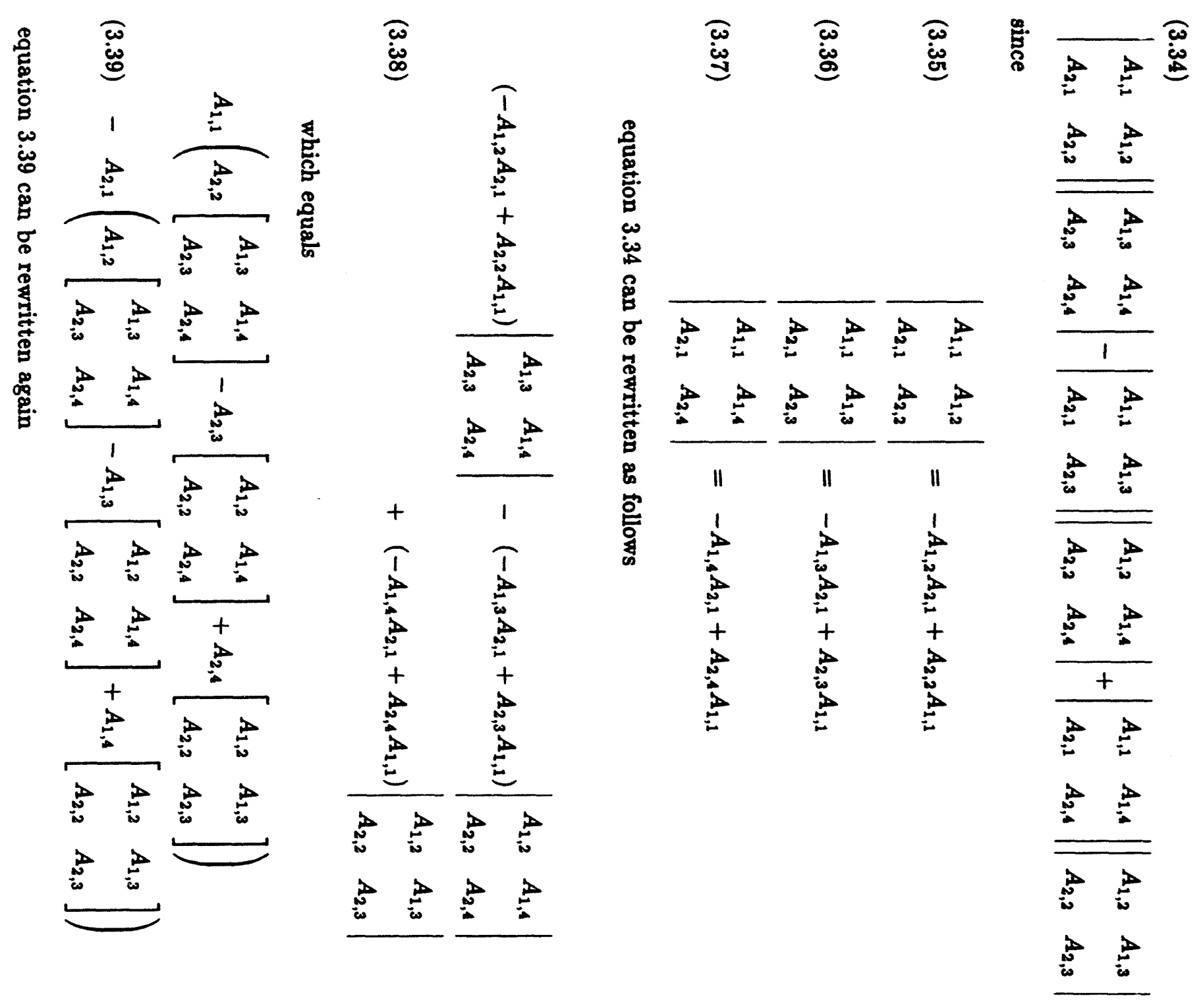




$$
\begin{aligned}
& A_{1,1}\left(\left[\begin{array}{ccc}
A_{2,2} & 0 & 0 \\
A_{1,2} & A_{1,3} & A_{1,4} \\
A_{2,2} & A_{2,3} & A_{2,4}
\end{array}\right]+\left[\begin{array}{ccc}
0 & A_{2,3} & 0 \\
A_{1,2} & A_{1,3} & A_{1,4} \\
A_{2,2} & A_{2,3} & A_{2,4}
\end{array}\right]+\left[\begin{array}{ccc}
0 & 0 & A_{2,4} \\
A_{1,2} & A_{1,3} & A_{1,4} \\
A_{2,2} & A_{2,3} & A_{2,4}
\end{array}\right]\right) \\
& (3.40)-A_{2,1}\left(\left[\begin{array}{ccc}
A_{1,2} & 0 & 0 \\
A_{1,2} & A_{1,3} & A_{1,4} \\
A_{2,2} & A_{2,3} & A_{2,4}
\end{array}\right]+\left[\begin{array}{ccc}
0 & A_{1,3} & 0 \\
A_{1,2} & A_{1,3} & A_{1,4} \\
A_{2,2} & A_{2,3} & A_{2,4}
\end{array}\right]+\left[\begin{array}{ccc}
0 & 0 & A_{1,4} \\
A_{1,2} & A_{1,3} & A_{1,4} \\
A_{2,2} & A_{2,3} & A_{2,4}
\end{array}\right]\right)
\end{aligned}
$$

which in turn equals

(3.41) $A_{1,1}\left[\begin{array}{ccc}A_{2,2} & A_{2,3} & A_{2,4} \\ A_{1,2} & A_{1,3} & A_{1,4} \\ A_{2,2} & A_{2,3} & A_{2,4}\end{array}\right]-A_{2,1}\left[\begin{array}{ccc}A_{1,2} & A_{1,3} & A_{1,4} \\ A_{1,2} & A_{1,3} & A_{1,4} \\ A_{2,2} & A_{2,3} & A_{2,4}\end{array}\right]=A_{1,1} * 0-A_{2,1} * 0=0$

Therefore, equation 3.34 is identically zero. In the " $\pi$ " notation this means that

$$
\pi_{1,2} \pi_{3,4}-\pi_{1,3} \pi_{2,4}+\pi_{1,4} \pi_{2,3}=0
$$

Equation 3.42 was generated from equation 3.32 by setting $I=(1)$ and $J=$ $(2,3,4)$. It is an easy excercise to see that the result is the same for any other $I$ and $J$ as long as $I \cap J=\emptyset$. If, however, $I \cap J \neq \emptyset$, where $I=(i)$ and $J=\left(i, j_{2}, j_{3}\right)$ then the resulting identity is trivial:

$$
\pi_{i, i} \pi_{j_{2}, j_{3}}-\pi_{i, j_{2}} \pi_{i, j_{3}}+\pi_{i, j_{3}} \pi_{i, j_{2}} \equiv 0
$$

3.2.1.2.3.2 Examples of Graßmann-Plücker Relations - $\mathbf{G ( 2 , 5 )} \quad G(2,5)$, however, is slightly more complicated. $G(2,5)$ is isomorphic to a dense subset of $\mathbb{C}^{\circ}$, and we shall see that there are $\left(\begin{array}{l}5 \\ 4\end{array}\right)=5$ nontrivial Plücker relations. But the Plücker map takes $G(2,5)$ into $\mathbb{P}^{9}$, so must be three independent Plücker relations corresponding to $\Lambda$ where 


$$
\Lambda=\left[\begin{array}{lllll}
A_{1,1} & A_{1,2} & A_{1,3} & -\ldots,: & A_{1,5} \\
A_{2,1} & A_{2,2} & A_{2,3} & A_{2,4} & A_{2,3}
\end{array}\right]
$$

As long as we choose four different columns of 3.44 it does not matter how the four columns are assigned to $I$ and $J$. There are $\left(\begin{array}{l}5 \\ 4\end{array}\right)=5$ ways to choose the columns

$$
\begin{array}{ll}
I_{1}=(1) & J_{1}=(2,3,4) \\
I_{2}=(1) & J_{2}=(2,3,5) \\
I_{3}=(1) & J_{3}=(2,4,5) \\
I_{4}=(1) & J_{4}=(3,4,5) \\
I_{5}=(2) & J_{5}=(3,4,5)
\end{array}
$$

corresponding to the five Plücker relations:

$$
\begin{aligned}
& \pi_{1,2} \pi_{3,4}-\pi_{1,3} \pi_{2,4}+\pi_{1,4} \pi_{2,3}=0 \\
& \pi_{1,2} \pi_{3,5}-\pi_{1,3} \pi_{2,5}+\pi_{1,5} \pi_{2,3}=0 \\
& \pi_{1,2} \pi_{4,5}-\pi_{1,4} \pi_{2,5}+\pi_{1,5} \pi_{2,4}=0 \\
& \pi_{1,3} \pi_{4,5}-\pi_{1,4} \pi_{3,5}+\pi_{1,5} \pi_{3,4}=0 \\
& \pi_{2,3} \pi_{4,5}-\pi_{2,4} \pi_{3,5}+\pi_{2,5} \pi_{3,4}=0
\end{aligned}
$$

3.2.1.2.3.3 More General Graßmann-Plücker Relations For there to be a nontrivial Graßmann relation, at least three of the " $j_{0}$ " must be distinct from the " $i_{0}$ " and one of the " $i_{e}$ " must be distinct from the " $j_{e}$ ". One can check that there may only be an even number of nonrepeated indices. Suppose for any $k<(n-1)$ and $\Lambda$ in $G(k, n)$, there are exactly four indices, $l_{1}, l_{2}, l_{3}$, and $l_{1}$, which are not repeated. Without loss of generality assume that $I=\left(i_{1}, i_{2}, \ldots, i_{(k-2)}, l_{1}\right)$ and $J=\left(i_{1}, i_{2}, \ldots, i_{(k-2)}, l_{2}, l_{3}, l_{4}\right)$. The Plücker-Graßmann relation generated by $I$ and $J$ is similar to the relation generated by an element of $G(2,4)$ where $I=\left(l_{1}\right), J=\left(l_{2}, l_{3}, l_{4}\right)$. The other indices don't "matter" because they contribute only zero terms to equation 3.32 . 
If we consider $G(k, n)$ for $k>2$ there are many identities given by 3.32. Some of these identitites have terms which are identically zero. (Whenever $j_{\lambda} \in i_{1}, i_{2}, \ldots, i_{k-1}$, for example.) Suppose $\Lambda$ represents an element of $G(k, n)$ and some of the columns of $\Lambda$ are repeated. If there are only six different columns in $\Lambda$ we can calculate the number of nontrivial Plücker-Graßmann relations as though we were working in $G(3,6)$. In $G(3,6)$, $I$ and $J$ have two and four components, respectively. So there are $\left(\begin{array}{l}8 \\ 4\end{array}\right)=15$ ways to pick $I$ and $J$ so that $I$ has two indices and $J$ has four indices. Hence there are 15 nontrivial Plücker-Graßmann relations. Note, however, that $G(3,6)$ is isomorphic to a dense subset of $\mathbb{C}^{(6-3)}=\mathbb{C}^{9}$ and that the Plücker-Graßmann map takes $G(3,6)$ into $\mathbb{P}^{\left(\frac{1}{3}\right)-1}=\mathbb{P}^{19}$. Hence, among the 15 Plücker-Graßmann relations, ten are independent. Plücker-Graßmann relations will first be used to simplify the solutions to the nonzero entries of $P_{i h}$ which were calculated in section 3.2.1.1.

\subsubsection{Simplifying solutions to $P_{i h}$ by adding Graßmann identities}

Notice that in 3.28 several of the coefficients in the numerator contain factors which are quadratic in minors of $Q$. Some of the identities used to embed $G(3,7)$ in $\left.\mathbb{P}_{3}^{(7)}\right)-1$ are useful in simplifying 3.28 . Consider the matrix:

$$
\left[\begin{array}{lllllll}
Q_{1,5} & Q_{1,6} & Q_{1,7} & Q_{1,8} & 1 & 0 & 0 \\
Q_{2,5} & Q_{2,6} & Q_{2,7} & Q_{2,8} & 0 & 1 & 0 \\
Q_{8,5} & Q_{8,6} & Q_{8,7} & Q_{8,8} & 0 & 0 & 1
\end{array}\right]
$$

The quadratic factors appearing in 3.28 are written below as they appear in 3.28 and in the $\pi$ notation used in section 3.2.1.2.3.

$$
\begin{aligned}
& d Q_{[1,2],[5,6]} d Q_{[1,2,8],[6,7,8]}-d Q_{[1,2],[6,7]} d Q_{[1,2,8],[5,6,8]}=\pi_{1,2,7} \pi_{2,3,4}-\pi_{2,3,7} \pi_{1,2,4} \\
& \mathrm{dQ}_{[1,2],[5,6]} \mathrm{dQ}_{[1,2,8],[5,7,8]}+\mathrm{d} \mathrm{Q}_{[1,2],[5,8]} \mathrm{d} \mathrm{Q}_{[1,2,8],[5,6,7]}=\pi_{1,2,7} \pi_{1,3,4}+\pi_{1,4,7} \pi_{1,2,3} \\
& \mathrm{dQ}_{[1,2],[5,6]} \mathrm{dQ} \mathrm{Q}_{[1,2,8],[5,7,8]}-\mathrm{d} \mathrm{Q}_{[1,2],[5,7]} \mathrm{d} \mathrm{Q}_{[1,2,8],[5,6,8]}=\pi_{1,2,7} \pi_{1,3,4}-\pi_{1,3,7} \pi_{1,2,4} \\
& d Q_{[1,2],[6,8]} d Q_{[1,2,8],[5,6,7]}+d Q_{[1,2],[5,6]} d Q_{[1,2,8],[6,7,8]}=\pi_{2,4,7} \pi_{1,2,3}+\pi_{1,2,7} \pi_{2,3,4}
\end{aligned}
$$

The following Graßmann identities may be used to simplify the equations above: 


$$
\begin{aligned}
& \pi_{1,2,7} \pi_{2,3,4}-\pi_{1,2,2} \pi_{7,3,4}+\pi_{1,2,3} \pi_{7,2,4}-\pi_{1,2,4} \pi_{7,2,3}=0 \\
& \pi_{1,2,7} \pi_{1,3,4}-\pi_{1,2,1} \pi_{7,3,4}+\pi_{1,2,3} \pi_{7,1,4}-\pi_{1,2,4} \pi_{7,1,3}=0 \\
& \pi_{1,2,7} \pi_{1,3,4}-\pi_{1,2,1} \pi_{7,3,4}+\pi_{1,2,3} \pi_{7,1,4}-\pi_{1,2,4} \pi_{7,1,3}=0 \\
& \pi_{2,4,7} \pi_{1,2,3}-\pi_{2,4,1} \pi_{7,2,3}+\pi_{2,4,2} \pi_{7,1,3}-\pi_{2,4,3} \pi_{7,1,2}=0
\end{aligned}
$$

So we can simplify the right hand sides of the quadratic clusters in 3.48 as follows

$$
\begin{aligned}
& \pi_{1,2,7} \pi_{2,3,4}-\pi_{2,3,7} \pi_{1,2,4}=-\pi_{2,4,7} \pi_{1,2,3} \\
& \pi_{1,2,7} \pi_{1,3,4}+\pi_{1,4,7} \pi_{1,2,3}=\pi_{1,2,4} \pi_{1,3,7} \\
& \pi_{1,2,7} \pi_{1,3,4}-\pi_{1,3,7} \pi_{1,2,4}=-\pi_{1,4,7} \pi_{1,2,3} \\
& \pi_{2,4,7} \pi_{1,2,3}+\pi_{1,2,7} \pi_{2,3,4}=\pi_{2,3,7} \pi_{1,2,4}
\end{aligned}
$$

The Graßmann relations in $\mathbf{3 . 5 0}$ allow us to make the following substitutions:

$$
\begin{aligned}
d Q_{[1,2],[5,6]} d Q_{[1,2,8],[6,7,8]}-d Q_{[1,2],[6,7]} d Q_{[1,2,8],[5,6,8]} & =-d Q_{[1,2],[6,8]} d Q_{[1,2,8],[5,6,7]} \\
d Q_{[1,2],[5,6]} d Q_{[1,2,8],[5,7,8]}+d Q_{[1,2],[5,8]} d Q_{[1,2,8],[5,6,7]} & =d Q_{[1,2],[5,7]} d Q_{[1,2,8],[5,6,8]} \\
(3.51) d Q_{[1,2],[5,6]} d Q_{[1,2,8],[5,7,8]}-d Q_{[1,2],[5,7]} d Q_{[1,2,8],[5,6,8]} & =-d Q_{[1,2],[5,8]} d Q_{[1,2,8],[5,6,7]} \\
d Q_{[1,2],[6,8]} d Q_{[1,2,8],[5,6,7]}+d Q_{[1,2],[5,6]} d Q_{[1,2,8],[6,7,8]} & =d Q_{[1,2],[6,7]} d Q_{[1,2,8],[5,6,8]}
\end{aligned}
$$

When 3.51 are substituted into the solution in 3.28 the resulting solution looks much simpler:

$$
\begin{aligned}
& s 12 s=\left(A_{8,7} A_{8,8}\left(\mathrm{dQ}_{[1,2],[6,8]} A_{6,6}+\mathrm{dQ} Q_{[1,2],[5,8]} A_{5,6}\right)\right. \\
& \left(-d Q_{[5,6,8],[1,2,8]} d Q_{[1,2],\{5,6]}+d Q_{[5,6],[1,2]} d Q_{[1,2,8],[5,6,8]}\right)+ \\
& \mathrm{dQ}_{[1,2],[6,8]}\left(\mathrm{dQ}_{[5,6],[1,2]} \mathrm{dQ} \mathrm{Q}_{[1,2,8],[5,6,7]}-\mathrm{dQ}_{[1,2],[5,6]} \mathrm{dQ} \mathrm{Q}_{[5,6,8],[1,2,7]}\right) A_{6,6} A_{7,8} A_{8,7}+ \\
& \mathrm{dQ}_{[1,2],[6,7]}\left(-\mathrm{dQ}_{[5,6,8],[1,2,8]} \mathrm{dQ} \mathrm{Q}_{[1,2],[5,6]}+\mathrm{dQ}_{[5,6],[1,2]} \mathrm{d} \mathrm{Q}_{[1,2,8],[5,6,8]}\right) A_{7,7} A_{6,6} A_{8,8}+ \\
& \mathrm{dQ}_{[1,2],[5,8]}\left(\mathrm{dQ}_{[5,6],[1,2]} \mathrm{d} \mathrm{Q}_{[1,2,8],[5,6,7]}-\mathrm{dQ}_{[1,2],\{5,6]} \mathrm{d} \mathrm{Q}_{[5,6,8],(1,2,7]}\right) A_{5,6} A_{8,7} A_{7,8}+ \\
& \mathrm{dQ}_{[1,2],[5,7]}\left(-\mathrm{dQ}_{[5,6,8],[1,2,8]} \mathrm{dQ} \mathrm{Q}_{[1,2],[5,6]}+\mathrm{dQ}_{[5,6],[1,2]} \mathrm{dQ} \mathrm{Q}_{[1,2,8],[5,6,8]}\right) A_{5,6} A_{8,8} A_{7,7}+ \\
& A_{7,8} A_{7,7}\left(\mathrm{dQ}_{[1,2],[6,7]} A_{6,6}+\mathrm{dQ}_{[1,2],[5,7]} A_{5,6}\right) \\
& \left.\left(d Q_{[5,6],[1,2]} d Q_{[1,2,8],[5,6,7]}-d Q_{[1,2],[5,6]} d Q_{[5,6,8],\{1,2,7]}\right)\right) / \\
& d A_{[7,8],[7,8]} \mathrm{dQ}_{[1,2],[7,8]} \mathrm{d} \mathrm{Q}_{[1,2],(5,6]} \mathrm{d} \mathrm{Q}_{[5,6],[1,2]}
\end{aligned}
$$

which factors to become 


$$
\begin{aligned}
& s 12 s=\left(\left(A_{8,8} \mathrm{dQ}_{[5,8],[1,2]} \mathrm{dQ} \mathrm{Q}_{[1,2,8],(5,6,8]}-A_{7,8} \mathrm{dQ}_{[1,2],[8,6]} \mathrm{dQ}_{[5,0,8],[1,2,7]}-\right.\right. \\
& \left.A_{8,8} \mathrm{dQ}_{[5,6,8],\{1,2,8]} \mathrm{dQ}_{[1,2],[5,6]}+A_{7,8} \mathrm{~d} Q_{[5,6],[1,2]} \mathrm{dQ} \mathrm{Q}_{[1,2,8],[5,0,7]}\right) \\
& \left(\mathrm{dQ}_{[1,2],[6,8]} A_{6,0} A_{8,7}+\mathrm{dQ}_{[1,2],[5,8]} A_{5,0} A_{8,7}\right. \\
& \left.\left.+\mathrm{dQ}_{[1,2],(6,7]} A_{7,7} A_{6,6}+\mathrm{dQ}_{[1,2],[5,7]} A_{6,6} A_{7,7}\right)\right) / \\
& d A_{[7,8],[7,8]} \mathrm{d} \mathrm{Q}_{[1,2],[7,8]} \mathrm{dQ} \mathrm{Q}_{[1,2],[5,6]} \mathrm{d} \mathrm{Q}_{[5,6],[1,2]}
\end{aligned}
$$

All of the sixteen solutions for the entries in $P_{i h}$ in terms of the entries of $A$ factor once they have been simplified using Plücker-Graßmann relations.

\subsubsection{A "special" model with a closed system of equations}

Motivated by the observation that the rank of the direct map is generically 48 , we looked for a model which has 48 independent parameters, distributed evenly among the four pixels. In order to reduce the number of independent parameters we made the following identifications:

$$
e i j e=w i j w, \text { sijs }=n i j n, \operatorname{sijn}=n i j s, \text { cijw }=w i j e \text { for all } i, j .
$$

The first two constraints are fairly natural. They represent instances of the principle of "microscopic reversibility". Notice that the other two conditions are a bit less natural; they represent a certain type of "mirror symmetry". Experimentalists often make use of physically plausible constraints. Unfortunately, these constraints do not always simplify the mathematical problem. For example, imposing microscopic reversibility on the system would lead to less than twelve free parameters per pixel. Data matrices generated by microscopically reversible $n \times n$ models are symmetric. This symmetry renders half of the otherwise independent consistency conditions redundant. Unfortunately, it also renders $n(n-1) / 2$ of the data redundant. In the $2 \times 2$ case this reduces the rank of the direct map to 28 . Finally notice that we have not made the assumption that the probability of being killed in a pixel is independent of the direction from which the photon entered the pixel. 
Once the above identifications are made, the problem has the following features: the rank of the forward map remains 48, the main diagonals of $P_{i o}$ and $P_{h h}$ are common, and the off diagonals of $P_{h o}$ and $P_{i h}$ are common.

3.2.1.4.1 Cubics and Quadratics In section 3.2.1.1 we had freedom to choose sixteen of the 32 equations in the matrix equation 3.16 which were functions only of $A$ and $P_{h h}$. More precisely, in each of the eight columns of equations in 3.16 we were free to choose two of the four equations which were independent of $P_{i o}$, and $P_{i h}$. Experimentation with a few of the $\left(\begin{array}{l}4 \\ 2\end{array}\right)^{8}=1,679,616$ possibilities showed that some choices of equations are better than others. Thanks to a different choice of equations the results presented below are somewhat simpler than those in $[5,6]$ and [7]. In this section, the solutions for the transition probabilities were obtained by disregarding the equations in 2.8 corresponding to the ' $x$ 's in the matrix below:

$$
\left[\begin{array}{llllllll}
0 & 0 & 0 & 0 & 0 & 0 & 0 & 0 \\
0 & 0 & 0 & 0 & 0 & 0 & 0 & 0 \\
x & 0 & 0 & 0 & 0 & x & x & x \\
x & 0 & 0 & 0 & 0 & x & x & x \\
0 & 0 & 0 & 0 & 0 & 0 & 0 & 0 \\
0 & 0 & 0 & 0 & 0 & 0 & 0 & 0 \\
0 & x & x & x & x & 0 & 0 & 0 \\
0 & x & x & x & x & 0 & 0 & 0
\end{array}\right]
$$

When substituting the solutions found in section 3.2.1.1 into 3.54 the equations corresponding to travel straight through a pixel yield eight quadratic identities. Many of the equations which follow are identically zero and the " $=0$ " has been omitted.

$$
\begin{aligned}
& \mathrm{dQ}_{[5,6],[7,8]}\left(\mathrm{dQ}_{[1,2],[5,6]} \mathrm{d} \mathrm{Q}_{[5,6,7],(1,2,7]} A_{8,7} A_{7,8}-\mathrm{d} Q_{[5,6],(1,2]} \mathrm{dQ}_{[1,2,7],[5,6,7]} A_{7,7} A_{8,8}\right. \\
& \left.+\left(\mathrm{dQ}_{[1,2],[5,6]} \mathrm{dQ} \mathrm{Q}_{[5,6,7],[1,2,8]}-\mathrm{dQ}_{[5,6],[1,2]} \mathrm{dQ} \mathrm{Q}_{[1,2,7],(5,6,8]}\right) A_{8,7} A_{8,8}\right) \\
& -\mathrm{dQ}_{[1,2],[5,6]} \mathrm{d} Q_{[5,6],[1,2]}\left(\mathrm{dQ}_{[5,6],[2,7]} A_{2,1} A_{7,7}+\mathrm{d} Q_{[5,6],[2,8]} A_{8,7} A_{2,1}\right. \\
& \left.+\mathrm{d} Q_{[5,6],[1,7]} A_{1,1} A_{7,7}+\mathrm{dQ} \mathrm{Q}_{[5,6],(1,8]} A_{8,7} A_{1,1}\right) \text {, }
\end{aligned}
$$


41

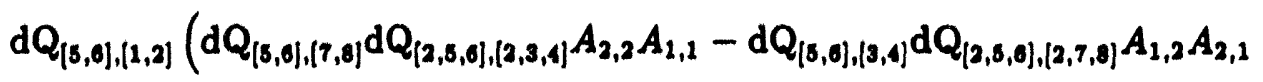

$$
\begin{aligned}
& \left.-\left(-\mathrm{d} \mathrm{Q}_{[3,0],(3,4]} \mathrm{d} \mathrm{Q}_{[2,8,0],[1,7,8]}+\mathrm{d} \mathrm{Q}_{[2,5,0],[1,3,4]} \mathrm{d} \mathrm{Q}_{[3,0],(7,8]}\right) A_{1,1} A_{1,2}\right) \\
& +\mathrm{dQ}_{[8,8],[7,8]} \mathrm{dQ} \mathrm{Q}_{[5,0],[3,4]}\left(\mathrm{dQ}_{[8,0],[1,8]} A_{8,8} A_{1,2}+\mathrm{dQ} \mathrm{Q}_{[8,0],[2,7]} A_{7,8} A_{2,2}\right. \\
& \left.+\mathrm{d} \mathrm{Q}_{[5,0],[1,7]} A_{7,8} A_{1,2}+\mathrm{d} Q_{[5,0],(2,0]} A_{8,8} A_{2,2}\right) \text {, }
\end{aligned}
$$

$$
\begin{aligned}
& \mathrm{dQ}_{[1,2],[5,6]}\left(\mathrm{dQ}_{[1,2],[7,8]} \mathrm{dQ} \mathrm{Q}_{[1,2,5],[3,4,8]} A_{6,6} A_{5,5}-\mathrm{dQ}_{[1,2],[3,4]} \mathrm{dQ} \mathrm{Q}_{[1,2,5],[3,7,8]} A_{6,8} A_{5,6}\right. \\
& \left.-\left(-\mathrm{dQ}_{[1,2],(3,4]} \mathrm{d} \mathrm{Q}_{[1,2,5],(0,7,8]}+\mathrm{d} \mathrm{Q}_{[1,2,5],(3,4,0]} \mathrm{d} \mathrm{Q}_{[1,2],(7,8)}\right) A_{6,6} A_{6,8}\right) \\
& -\mathrm{dQ}_{[1,2],[3,4]} \mathrm{dQ} \mathrm{Q}_{[1,2],[7,8]}\left(\mathrm{dQ}_{[1,2],[5,7]} A_{7,7} A_{5,5}+\mathrm{dQ} \mathrm{Q}_{[1,2],[5,8]} A_{8,7} A_{5,5}\right.
\end{aligned}
$$

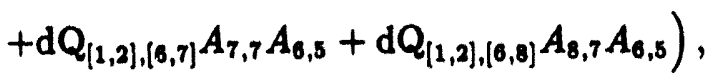

$$
\begin{aligned}
& \mathrm{dQ}_{[1,2],[3,4]}\left(\mathrm{dQ}_{[1,2],[3,6]} \mathrm{dQ} Q_{[3,5,6],[1,2,3]} A_{3,3} A_{4,4}-\mathrm{dQ}_{[5,0],[1,2]} \mathrm{dQ}_{[1,2,3],[3,5,6]} A_{4,3} A_{3,4}\right. \\
& \left.-\left(-\mathrm{dQ}_{[3,0],[1,2]} \mathrm{d} \mathrm{Q}_{[1,2,3],[4,5,0]}+\mathrm{d} \mathrm{Q}_{[3,5,0],[1,2,4]} \mathrm{d} \mathrm{Q}_{[1,2],[5,0]}\right) A_{4,4} A_{4,3}\right) \\
& -\mathrm{dQ}_{[1,2],[5,8]} \mathrm{dQ} \mathrm{Q}_{[5,6],[1,2]}\left(\mathrm{dQ}_{[1,2],[4,5]} A_{5,5} A_{4,3}+A_{4,3} A_{6,5} \mathrm{dQ} \mathrm{Q}_{[1,2],[1,0]}\right. \\
& \left.+\mathrm{dQ}_{[1,2],[3,0]} A_{0,5} A_{3,3}+\mathrm{dQ} Q_{[1,2],[3,5]} A_{5,5} A_{3,3}\right) \text {, }
\end{aligned}
$$

$$
\begin{aligned}
& d Q_{[5,0],[3,4]}\left(\left(d Q_{[5,6],[1,2]} d Q_{[1,2,4],[3,5,6]}-d Q_{[1,2],[5,6]} d Q_{[4,5,0],[1,2,3]}\right) A_{3,4} A_{3,3}\right. \\
& \left.-\mathrm{dQ}_{[1,2],[5,0]} \mathrm{dQ}_{[4,5,6],[1,2,4]} A_{4,3} A_{3,4}+\mathrm{dQ}_{[5,0],[1,2]} \mathrm{dQ}_{[1,2,4],[4,5,6]} A_{3,3} A_{4,4}\right)- \\
& \mathrm{dQ}_{[1,2],[5,6]} \mathrm{dQ}_{[3,6],(1,2]}\left(\mathrm{dQ}_{[5,6],[1,4]} A_{4,4} A_{1,2}+\mathrm{dQ}_{[5,6],(2,4)} A_{4,4} A_{2,2}+\right. \\
& \left.\mathrm{dQ}_{[5,0],[1,3]} A_{1,2} A_{3,4}+\mathrm{dQ}_{[5,0],(2,3]} A_{2,2} A_{3,4}\right)
\end{aligned}
$$

$$
\begin{aligned}
& \mathrm{dQ}_{[1,2],[5,6]}\left(-\mathrm{dQ}_{[1,2],[3,4]} \mathrm{dQ} Q_{[1,2,6],(6,7,8]} A_{6,6} A_{5,5}+\mathrm{dQ}_{[1,2],[7,8]} \mathrm{dQ}_{[1,2,6],[3,4,6]} A_{6,5} A_{5,6}\right. \\
& \left.+\left(\mathrm{dQ}_{[1,2,6],(3,4,5]} \mathrm{d} \mathrm{Q}_{[1,2],(7,8]}-\mathrm{dQ}_{[1,2],[3,4]} \mathrm{d} \mathrm{Q}_{[1,2,6],[3,7,8]}\right) A_{5,8} A_{5,5}\right) \\
& +\mathrm{dQ}_{[1,2],[3,4]} \mathrm{dQ}_{[1,2],[7,8]}\left(A_{4,4} A_{6,6} \mathrm{dQ}_{[1,2],[4,6]}+\mathrm{dQ}_{[1,2],[4,8]} A_{4,4} A_{5,6}\right. \\
& \left.+\mathrm{dQ}_{[1,2],[3,6]} A_{6,6} A_{3,4}+\mathrm{dQ}_{[1,2],[3,5]} A_{3,4} A_{5,6}\right) \text {, }
\end{aligned}
$$




$$
\begin{aligned}
& \mathrm{d} Q_{[5,0],[1,2]}\left(-\mathrm{d} Q_{[8,0],[3,4]} \mathrm{d} Q_{[1,5,0],(1,7,8]} A_{2,2} A_{1,1}+A_{2,1} A_{1,2} \mathrm{~d} Q_{[1,5,0],[1,3,4]} \mathrm{d} Q_{[5,0],[7,8]}\right. \\
& \left.+\left(\mathrm{dQ}_{[3,0],(7,8]} \mathrm{d} \mathrm{Q}_{[1,8,0),[2,3,4]}-\mathrm{dQ}_{[1,8,0),(2,7,8]} \mathrm{d} \mathrm{Q}_{[3,0],(3,4]}\right) A_{2,1} A_{2,2}\right) \\
& +\mathrm{dQ}_{[5,0],\{7,8]} \mathrm{d} Q_{[5,0],[3,4]}\left(\mathrm{dQ}_{[8,0],\{2,4]} A_{4,3} A_{2,1}+\mathrm{d} Q_{[8,0],(2,3]} A_{3,3} A_{2,1}\right. \\
& \left.+\mathrm{dQ}_{[\mathrm{b}, 0],[1,4]} A_{1,3} A_{1,1}+\mathrm{dQ}_{[3,0],[1,3]} A_{3,3} A_{1,1}\right) \text {, }
\end{aligned}
$$

and finally

$$
\begin{aligned}
& \mathrm{dQ}_{[1,2],[7,8]}\left(\mathrm{dQ}_{[3,0],[1,2]} \mathrm{d} Q_{[1,2,8],[5,0,8]} A_{8,7} A_{7,8}-\mathrm{d} Q_{[1,2],[3,0]} \mathrm{dQ}_{[5,0,8],[1,2,8]} A_{7,7} A_{8,8}\right. \\
& \left.+\left(\mathrm{dQ}_{[5,0],[1,2]} \mathrm{d} \mathrm{Q}_{[1,2,8],[5,6,7]}-\mathrm{dQ}_{[1,2],(5,0]} \mathrm{d} Q_{[5,0,8],[1,2,7]}\right) A_{7,7} A_{7,8}\right) \\
& +\mathrm{dQ}_{[1,2],[3,0]} \mathrm{dQ}_{[5,0],[1,2]}\left(\mathrm{dQ}_{[1,2],[3,8]} A_{8,8} A_{5,6}+\mathrm{dQ}_{[1,2],[6,7]} A_{6,0} A_{7,8}\right. \\
& \left.+\mathrm{dQ}_{[1,2],[3,7]} A_{5,6} A_{7,8}+\mathrm{dQ}_{[1,2],[6,8]} A_{8,8} A_{6,6}\right)
\end{aligned}
$$

The identifications corresponding to reversing direction inside a pixel yield eight cubic equations:

$$
\begin{aligned}
& -A_{2,1} \mathrm{~d} Q_{[5,6],(1,2]} \mathrm{dQ}_{[3,6],[7,8]} \mathrm{d} Q_{[5,6],[3,4]}- \\
& \mathrm{dQ}_{[5,0],[2,3]}\left(\mathrm{dQ}_{[1,8,0],[1,3,4]} \mathrm{d} \mathrm{Q}_{[5,0],[7,8]}-\mathrm{dQ}_{[1,5,0],(1,7,8]} \mathrm{dQ} \mathrm{Q}_{[5,0],[3,4]}\right) A_{3,3} A_{2,2} A_{1,1}- \\
& \mathrm{dQ}_{[5,0],(2,4]}\left(\mathrm{dQ}_{[1,5,0],(1,3,4]} \mathrm{d} \mathrm{Q}_{[5,0],[7,8]}-\mathrm{d} \mathrm{Q}_{[1,5,0),[1,7,8]} \mathrm{d} \mathrm{Q}_{[5,6],[3,4]}\right) A_{4,3} A_{2,2} A_{1,1}- \\
& \mathrm{dQ}_{[5,6],[1,3]}\left(\mathrm{dQ}_{[1,5,6],[1,3,4]} \mathrm{dQ} \mathrm{Q}_{[5,6],[7,8]}-\mathrm{d} \mathrm{Q}_{[1,5,6],[1,7,8]} \mathrm{d} \mathrm{Q}_{[5,6],(3,4]}\right) A_{1,2} A_{1,1} A_{3,3}- \\
& \mathrm{d} Q_{[5,0],[1,4]}\left(\mathrm{dQ}_{[1,3,0],[1,3,4]} \mathrm{d} Q_{[5,8],[7,8]}-\mathrm{dQ}_{[1,5,0],[1,7,8]} \mathrm{d} Q_{[5,6],(3,4]}\right) A_{1,2} A_{4,3} A_{1,1}- \\
& \mathrm{dQ}_{[5,0],[2,3]}\left(\mathrm{dQ}_{[5,0],(7,8)} \mathrm{d} \mathrm{Q}_{[1,5,0],[2,3,4]}-\mathrm{dQ}_{[1,5,0],[2,7,8]} \mathrm{d} \mathrm{Q}_{[5,0],(3,4]}\right) A_{2,1} A_{3,3} A_{2,2}- \\
& \mathrm{dQ}_{[5,6],[2,4]}\left(\mathrm{dQ}_{[5,6],[7,8]} \mathrm{d} Q_{[1,5,6],[2,3,4]}-\mathrm{d} Q_{[1,5,6],[2,7,8]} \mathrm{dQ} Q_{[5,6],[3,4]}\right) A_{4,3} A_{2,1} A_{2,2}- \\
& \mathrm{dQ}_{[5,0],[1,4]}\left(\mathrm{dQ}_{[5,0],(7,8)} \mathrm{d} \mathrm{Q}_{[1,5,0],[2,3,4]}-\mathrm{dQ}_{[1,5,6],[2,7,8]} \mathrm{dQ} \mathrm{Q}_{[5,0],(3,4]}\right) A_{1,2} A_{4,3} A_{2,1}
\end{aligned}
$$

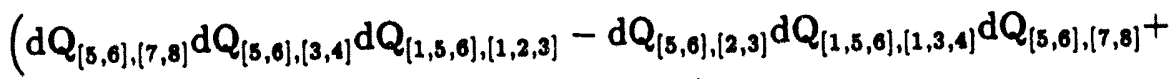

$$
\begin{aligned}
& \left.\mathrm{dQ}_{[5,6],[1,3]} \mathrm{dQ} Q_{[5,6],[3,4]} \mathrm{dQ} Q_{[1,5,6],[2,7,8]}\right) A_{1,2} A_{2,1} A_{3,3}
\end{aligned}
$$


43

$$
\begin{aligned}
& A_{6,8} \mathrm{dQ}_{[1,2],[3,0]} \mathrm{d} Q_{[1,2],(3,4]} \mathrm{d} Q_{[1,2],(7,8]} \\
& +\mathrm{dQ}_{[1,2],(3,8)}\left(-\mathrm{dQ}_{[1,2,5],\{3,4,5]} \mathrm{dQ}_{[1,2],\{7,8]}+\mathrm{dQ}_{[1,2],\{3,4]} \mathrm{dQ}_{[1,2,8],(3,7,8)}\right) A_{5,8} A_{8,7} A_{5,8} \\
& +\left(d Q_{[1,2],[3,4]} d Q_{[1,2],[7,8]} d Q_{[1,2,8],(3,0,8]}-d Q_{[1,2],[0,8]} d Q_{[1,2],[7,8]} d Q_{[1,2,8],[3,4,8]}+\right. \\
& \left.\mathrm{dQ}_{[1,2],(5,8]} \mathrm{dQ}_{[1,2],[3,4]} \mathrm{dQ} \mathrm{Q}_{[1,2,3),(6,7,8]}\right) A_{8,7} A_{0,8} A_{8,8} \\
& +\mathrm{dQ}_{[1,2],(6,8]}\left(\mathrm{dQ}_{[1,2],\{3,4]} \mathrm{d} \mathrm{Q}_{[1,2,5),(6,7,8]}-\mathrm{dQ}_{[1,2,8],[3,4,0]} \mathrm{d} \mathrm{Q}_{[1,2],[7,8]}\right) A_{6,5} A_{8,7} A_{6,0}
\end{aligned}
$$

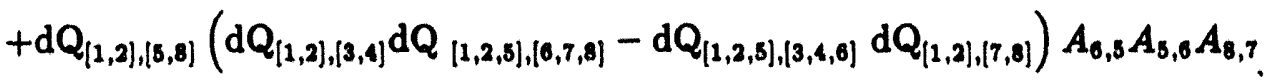

$$
\begin{aligned}
& +\mathrm{dQ}_{[1,2],[6,7]}\left(-\mathrm{dQ}_{[1,2,5],(3,4,5]} \mathrm{d} \mathrm{Q}_{[1,2],[7,8]}+\mathrm{dQ}_{[1,2],[3,4]} \mathrm{d} \mathrm{Q}_{[1,2,5],(5,7,8]}\right) A_{7,7} A_{6,6} A_{5,5} \\
& +\mathrm{dQ}_{[1,2],(8,7]}\left(-\mathrm{dQ}_{[1,2,5],[3,4,5]} \mathrm{dQ}_{[1,2],[7,8]}+\mathrm{dQ}_{[1,2],[3,4]} \mathrm{dQ}_{[1,2,5],\{5,7,8]}\right) A_{5,6} A_{7,7} A_{5,5} \\
& +\mathrm{dQ}_{[1,2],[6,7]}\left(\mathrm{dQ}_{[1,2],[3,4]} \mathrm{dQ}_{[1,2,8],[0,7,8]}-\mathrm{dQ}_{[1,2,8],[3,4,0]} \mathrm{dQ}_{[1,2],[7,8]}\right) A_{6,8} A_{6,0} A_{7,7} \\
& -\left(d Q_{[1,2],\{3,4]} d Q_{[1,2],[7,8]} d Q_{[1,2,5],(5,0,7]}+d Q_{[1,2],(3,7]} d Q_{[1,2],[7,8]} d Q_{[1,2,5],(3,4,0]}-\right. \\
& \left.\mathrm{dQ}_{[1,2],(0,7]} \mathrm{dQ}_{[1,2],[3,4]} \mathrm{dQ}_{[1,2,5],(6,7,8]}\right) A_{0,5} A_{8,0} A_{7,7},
\end{aligned}
$$

$$
\begin{aligned}
& -A_{5,8} \mathrm{dQ}_{[1,2],[3,6]} \mathrm{d} \mathrm{Q}_{[1,2],[3,4]} \mathrm{d} \mathrm{Q}_{[1,2],[7,8]} \\
& +\mathrm{dQ}_{[1,2],[3,8]}\left(-\mathrm{d} \mathrm{Q}_{[1,2,6],[3,4,8]} \mathrm{dQ} \mathrm{[1,2],[7,8]}+\mathrm{dQ}_{[1,2],[3,4]} \mathrm{d} \mathrm{Q}_{[1,2,6],[3,7,8]}\right) A_{3,4} A_{5,8} A_{5,6} \\
& +\mathrm{dQ}_{[1,2],[4,6]}\left(-\mathrm{dQ}_{[1,2,0],(3,4,5]} \mathrm{d} \mathrm{Q}_{[1,2],[7,8]}+\mathrm{dQ}_{[1,2],[3,4]} \mathrm{d} \mathrm{Q}_{[1,2,6],[5,7,8]}\right) A_{4,4} A_{5,6} A_{6,5} \\
& +\mathrm{dQ}_{[1,2],\{4,0)}\left(-\mathrm{dQ}_{[1,2,0],[3,4,6]} \mathrm{d} \mathrm{Q}_{[1,2],[7,8]}+\mathrm{d} \mathrm{Q}_{[1,2],[3,4]} \mathrm{d} \mathrm{Q}_{[1,2,6],(6,7,8]}\right) A_{6,6} A_{4,4} A_{6,5} \\
& +\mathrm{dQ}_{[1,2],[4,5]}\left(-\mathrm{d} \mathrm{Q}_{[1,2,0],[3,4,5]} \mathrm{dQ} \mathrm{Q}_{[1,2],[7,8]}+\mathrm{dQ}_{[1,2],[3,4]} \mathrm{d} \mathrm{Q}_{[1,2,0],[5,7,8]}\right) A_{5,5} A_{4,4} A_{5,6}
\end{aligned}
$$

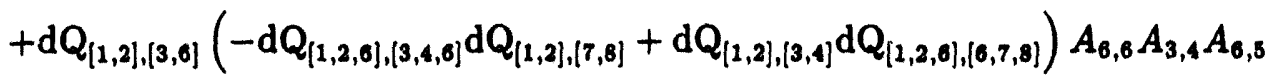

$$
\begin{aligned}
& -\left(d Q_{[1,2],[3,4]} d Q_{[1,2],[7,8]} d Q_{[1,2,6],[4,5,6]}-d Q_{[1,2],[4,5]} d Q_{[1,2],[3,4]} d Q_{[1,2,6],(6,7,8]}+\right. \\
& \left.\mathrm{dQ}_{[1,2],[4,6]} \mathrm{dQ}_{[1,2],[7,8]} \mathrm{dQ}_{[1,2,6],[3,4,5]}\right) A_{4,4} A_{6,6} A_{5,5} \\
& +\left(d Q_{[1,2],(3,4]} d Q_{[1,2],[7,8]} d Q_{[1,2,6],(3,5,6]}+d Q_{[1,2],[3,6]} d Q_{[1,2],[3,4]} d Q_{[1,2,6],[3,7,8]}-\right. \\
& \left.\mathrm{dQ}_{[1,2],(3,5]} \mathrm{dQ}_{[1,2],[7,8]} \mathrm{dQ}_{[1,2,6],(3,4,6]}\right) A_{6,5} A_{5,8} A_{3,4} \\
& -\left(d Q_{[1,2],[3,4]} d Q_{[1,2],[7,8]} d Q_{[1,2,6],\{3,5,6]}-d Q_{[1,2],(3,8]} d Q_{[1,2],(3,4]} d Q_{[1,2,6],[6,7,8]}+\right. \\
& \left.\mathrm{dQ}_{[1,2],(3,8]} \mathrm{dQ}_{[1,2],[7,8]} \mathrm{dQ}_{[1,2,6],(3,4,5]}\right) A_{3,4} A_{6,8} A_{5,5}
\end{aligned}
$$


44

$$
\begin{aligned}
& -A_{7,8} \mathrm{dQ}_{[1,2],[7,0]} \mathrm{d} Q_{[1,2],[3,0]} \mathrm{d} Q_{[8,0],[1,2]} \\
& -\mathrm{dQ}_{[1,2],[0,7]}\left(\mathrm{dQ}_{[8,0],[1,2]} \mathrm{d} \mathrm{Q}_{[1,2,8],[8,0,7]}-\mathrm{d} \mathrm{Q}_{[1,2],[8,0]} \mathrm{d} \mathrm{Q}_{[8,0,8],[1,2,7]}\right) A_{7,7} A_{6,8} A_{7,8}
\end{aligned}
$$

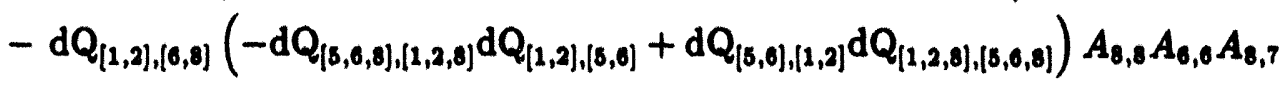

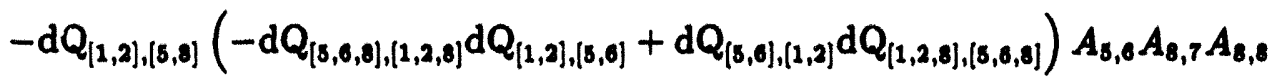

$$
\begin{aligned}
& -\mathrm{dQ}_{[1,2],[5,8]}\left(\mathrm{dQ}_{[5,0],[1,2]} \mathrm{d} \mathrm{Q}_{[1,2,8),(3,6,7]}-\mathrm{dQ}_{[1,2],[5,0]} \mathrm{dQ}_{[5,0,8],(1,2,7]}\right) A_{8,0} A_{8,7} A_{7,8} \\
& -d Q_{[1,2],(0,8]}\left(d Q_{[8,0],(1,2]} d Q_{[1,2,8],(5,0,7]}-d Q_{[1,2],[5,0]} d Q_{[5,6,8],[1,2,7]}\right) A_{6,0} A_{7,8} A_{8,7} \\
& -d Q_{[1,2],[6,7]}\left(-d Q_{[8,0,8],[1,2,8]} d Q_{[1,2],[5,0]}+d_{[3,0],[1,2]} d Q_{[1,2,8],[5,6,8]}\right) A_{7,7} A_{8,0} A_{8,8}
\end{aligned}
$$

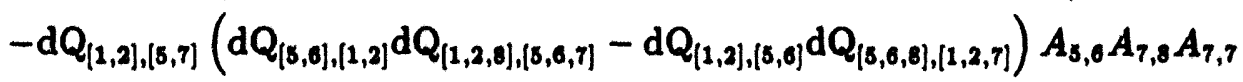

$$
\begin{aligned}
& -\mathrm{dQ}_{[1,2],[5,7]}\left(-\mathrm{dQ}_{[5,6,8],[1,2,8]} \mathrm{dQ} \mathrm{Q}_{[1,2],[3,6]}+\mathrm{dQ}_{[5,0],[1,2]} \mathrm{dQ} \mathrm{Q}_{[1,2, \mathrm{~d}],[5,6,8]}\right) A_{5,0} A_{8,8} A_{7,7}
\end{aligned}
$$

$$
\begin{aligned}
& A_{4,3} \mathrm{dQ}_{[1,2],[3,4]} \mathrm{dQ}_{[1,2],(5,0]} \mathrm{dQ}_{[3,0],[1,2]}
\end{aligned}
$$

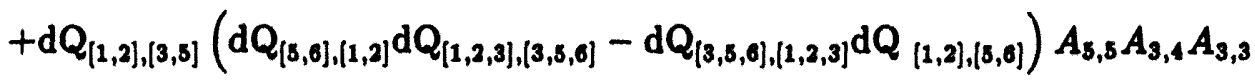

$$
\begin{aligned}
& +\mathrm{d} Q_{[1,2],[3,5]}\left(\mathrm{dQ}_{[5,06,[1,2]} \mathrm{d} \mathrm{Q}_{[1,2,3],[4,5,0]}-\mathrm{dQ}_{[3,5,0],[1,2,4]} \mathrm{d} \mathrm{Q}_{[1,2],[5,0]}\right) A_{4,3} A_{5,5} A_{3,4} \\
& +\mathrm{dQ}_{[1,2],[3,6]}\left(\mathrm{dQ}_{[3,6],(1,2]} \mathrm{d} \mathrm{Q}_{[1,2,3],[3,5,6]}-\mathrm{d} \mathrm{Q}_{[3,5,6],[1,2,3]} \mathrm{d} \mathrm{Q}_{[1,2],[3,6]}\right) A_{3,4} A_{6,5} A_{3,3} \\
& +\mathrm{dQ}_{[1,2],[4,6]}\left(\mathrm{dQ} \mathrm{[5,0],[1,2]} \mathrm{d} \mathrm{Q}_{[1,2,3],[3,5,0]}-\mathrm{dQ}_{[3,5,0],[1,2,3]} \mathrm{dQ} \mathrm{Q}_{[1,2],[5,0]}\right) A_{4,4} A_{3,3} A_{6,5} \\
& +\mathrm{dQ}_{[1,2],[4,0]}\left(\mathrm{dQ}_{[5,0],[1,2]} \mathrm{d} \mathrm{Q}_{[1,2,3],[4,5,0]}-\mathrm{dQ}_{[3,5,0],[1,2,4]} \mathrm{d} \mathrm{Q}_{[1,2],[5,0]}\right) A_{6,5} A_{4,4} A_{4,3} \\
& +\mathrm{d} Q_{[1,2],[4,5]}\left(\mathrm{dQ}_{[5,0],[1,2]} \mathrm{d} \mathrm{Q}_{[1,2,3],[4,5,0]}-\mathrm{dQ}_{[3,5,0],[1,2,4]} \mathrm{d} \mathrm{Q}_{[1,2],[5,0]}\right) A_{5,5} A_{4,4} A_{4,3} \\
& +\left(d Q_{[5,0],\{1,2]} d Q_{[1,2],\{5,8]} d Q_{[1,2,3],\{3,4,5]}-d Q_{[1,2],[4,5]} d Q_{[1,2],[5,0]} d Q_{[3,5,0],[1,2,3]}+\right. \\
& \left.\mathrm{dQ}_{[1,2],[3,5]} \mathrm{dQ}_{[5,6],[1,2]} \mathrm{dQ}_{[1,2,3,[4,5,8]}\right) A_{5,5} A_{3,3} A_{4,4} \\
& -\left(\mathrm{dQ}_{[3,6],(1,2]} \mathrm{d} \mathrm{Q}_{[1,2],[5,0]} \mathrm{d} \mathrm{Q}_{[1,2,3],[3,4,6]}+\mathrm{d} \mathrm{Q}_{[1,2],[3,6]} \mathrm{d} \mathrm{Q}_{[1,2],[5,0]} \mathrm{d} \mathrm{Q}_{[3,8,6],[1,2,4]}\right. \\
& \left.-\mathrm{dQ}_{[1,2],[4,0]} \mathrm{dQ}_{[8,0],[1,2]} \mathrm{dQ} \mathrm{Q}_{[1,2,3],(3,5,0]}\right) A_{4,3} A_{3,4} A_{6,5} \text {, }
\end{aligned}
$$


45

$$
\begin{aligned}
& A_{3,4} \mathrm{dQ}_{[(5,0),(3,4)} \mathrm{d} Q_{[1,2],(8,0)} \mathrm{d} Q_{[5,0],(1,2]} \\
& -d Q_{[8,0],(2,3]}\left(d Q_{[8,0],(1,2]} d Q_{[1,2,4],(4,8,0]}-d Q_{[1,2],(8,0]} d Q_{[4,8,0],[1,2,4]}\right) A_{2,2} A_{3,3} A_{4,4}
\end{aligned}
$$

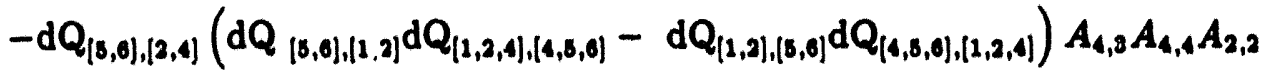

$$
\begin{aligned}
& +\left(d Q_{[8,0],[1,2]} d Q_{[1,2],(8,0]} d Q_{[1,3,0],(1,3,4]}-d Q_{[8,0],(1,3]} d Q_{[3,0],(1,2]} d Q_{[1,2,4],(1,8,0]}\right. \\
& \left.+\mathrm{dQ}_{[5,0],(1,4]} \mathrm{d} Q_{[1,2],(8,0]} \mathrm{d} Q_{[4,8,0],(1,2,3]}\right) A_{1,2} A_{4,4} A_{3,3} \\
& -\mathrm{dQ}_{[3,0],(1,4)}\left(\mathrm{dQ}_{[3,0],(1,2]} \mathrm{d} \mathrm{Q}_{[1,2,4),(4,5,0]}-\mathrm{dQ}_{[1,2],[3,0]} \mathrm{d} \mathrm{Q}_{[4,3,0],(1,2,4]}\right) A_{4,3} A_{1,2} A_{4,4} \\
& -d Q_{[5,0),(2,3]}\left(\mathrm{dQ}_{[3,0],(1,2]} \mathrm{d} Q_{[1,2,4),(3,5,0]}-\mathrm{dQ}_{[1,2],(3,0]} \mathrm{d} Q_{[4,3,0],(1,2,3]}\right) A_{3,4} A_{2,2} A_{3,3}
\end{aligned}
$$

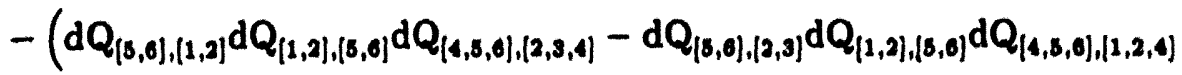

$$
\begin{aligned}
& \left.+\mathrm{dQ}_{[3,0],[2,4]} \mathrm{dQ} \mathrm{Q}_{[3,0],[1,2]} \mathrm{dQ} \mathrm{Q}_{[1,2,4],(3,8,0]}\right) A_{4,3} A_{3,4} A_{2,2}
\end{aligned}
$$

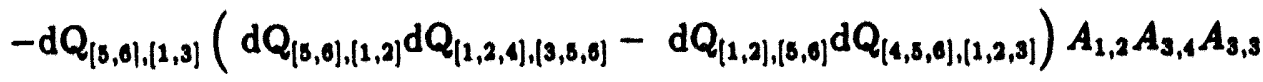

$$
\begin{aligned}
& -d Q_{[y, 0],(1,4]}\left(d Q_{[5,0],(1,2]} d Q_{[1,2,4],(3,5,0]}-d Q_{[1,2],(8,0]} d Q_{[4,3,0),(1,2,3]}\right) A_{4,3} A_{1,2} A_{3,4} \text {, }
\end{aligned}
$$

$$
\begin{aligned}
& -\lambda_{8,7} d Q_{[(5,0),[7,8]} d Q_{[3,6],[1,2]} d Q_{[1,2],[5,0]} \\
& +\left(d Q_{[3,0],(1,2]} d Q_{[1,2],(3,6])} d Q_{[5,0,7],(1,7,8]}-d Q_{[5,0],(1,7]} d Q_{[5,0],(1,2]} d Q_{[1,2,7],(3,0,8]}+\right.
\end{aligned}
$$

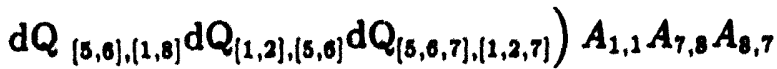

$$
\begin{aligned}
& -\mathrm{dQ}_{[3,0],[1,8]}\left(\mathrm{dQ}_{[3,0],[1,2]} \mathrm{d} \mathrm{Q}_{[1,2,7],[5,0,8]}-\mathrm{d} \mathrm{Q}_{[1,2],[(, 0]} \mathrm{d} \mathrm{Q}_{[5,0,7],[1,2,8]}\right) A_{8,8} A_{1,1} A_{8,7} \\
& -\mathrm{dQ}_{[5,0],\{1,7]}\left(-\mathrm{dQ}_{[1,2],(5,0]} \mathrm{dQ} \mathrm{Q}_{[5,6,7],(1,2,7]}+\mathrm{dQ}_{[3,0],\{1,2]} \mathrm{dQ} \mathrm{Q}_{[1,2,7],[5,0,7]}\right) A_{7,7} A_{1,1} A_{7,8} \\
& -\left(d Q_{[5,6],\{1,2]} d Q_{[1,2],(5,6]} d Q_{[5,6,7],(1,7,8)}-d Q_{[5,0],(1,7]} d Q_{[1,2],\{5,6]} d Q_{[5,0,7],[1,2,8]}\right. \\
& \left.+\mathrm{dQ}_{[5,6],(1,8]} \mathrm{dQ} \mathrm{Q}_{[8,6],(1,2]} \mathrm{dQ} \mathrm{Q}_{[1,2,7],[8,0,7]}\right) A_{8,8} A_{7,7} A_{1,1}
\end{aligned}
$$

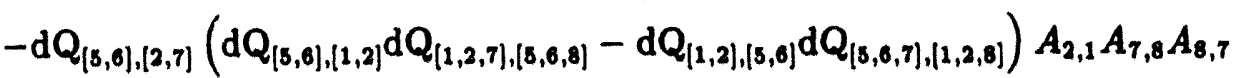

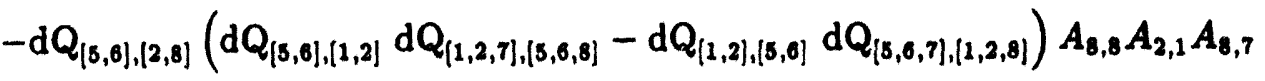

$$
\begin{aligned}
& -\mathrm{dQ}_{[3,6],[2,7]}\left(-\mathrm{d} Q_{[1,2],[5,0]} \mathrm{d} \mathrm{Q}_{[5,0,7],\{1,2,7]}+\mathrm{dQ}_{[5,0],[1,2]} \mathrm{d} Q_{[1,2,7],[5,0,7]}\right) A_{7,7} A_{2,1} A_{7,8} \\
& -\left(d Q_{[5,6],[1,2]} d Q_{[1,2],(5,6]} d Q_{[5,6,7],\{2,7,8]}-d Q_{[5,0],\{2,7]} d Q_{[1,2],(5,6]} d Q_{[5,6,7],\{1,2,8]}\right. \\
& +\mathrm{dQ}_{[3,6],[2,8]} \mathrm{d} \mathrm{Q}_{[3,6],[1,2]} \mathrm{d} \mathrm{Q}_{[1,2,7],(5,6,7])} A_{8,8} A_{7,7} A_{2,1} \text {, }
\end{aligned}
$$

and 
46

(3.71)

$$
\begin{aligned}
& A_{1,2} \mathrm{dQ}_{[3,0],(1,2]} \mathrm{dQ} \mathrm{Q}_{[3,0),(7,8]} \mathrm{d} \mathrm{Q}_{[3,0],(3,4]} \\
& +\left(d Q_{[3,0],(7,0)} d Q_{[8,0],(3,4]} d Q_{[2,8,0],(1,2,7]}+d Q_{[3,0],(1,7]} d Q_{[3,0],(7,0]} d Q_{[2,8,0],(2,3,4]}\right. \\
& \left.-\mathrm{dQ}_{[3,0],(2,7]} \mathrm{d} \mathrm{Q}_{[3,0],(3,4]} \mathrm{d} \mathrm{Q}_{[2,3,0],[1,7,8]}\right) A_{7,8} A_{2,2} A_{1,1} \\
& +d Q_{[3,0],(1,7]}\left(-d Q_{[3,0),(3,4]} d Q_{[2,8,0],(1,7,0]}+d Q_{[2,8,0],(1,3,4)} d Q_{[3,0],(7,0)}\right) A_{1,2} A_{1,1} A_{7,8} \\
& +\left(d Q_{[8,0],[7,8]} d Q_{[8,0],(3,4]} d Q_{[2,8,0],(2,2,8]}+d Q_{[5,0],[1,0]} d Q_{[3,0],[7,8]} d Q_{[2,5,0],[2,3,0]}-\right. \\
& \left.\mathrm{dQ}_{[3,0],(2,0]} \mathrm{d} Q_{[3,0],(3,4]} \mathrm{d} Q_{[2,5,0],(1,7,8)}\right) A_{8,8} A_{2,2} A_{1,1}
\end{aligned}
$$

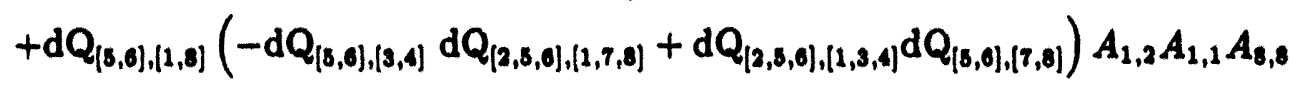

$$
\begin{aligned}
& +d Q_{[3,0],[2,7]}\left(\mathrm{dQ}_{[3,0],[7,8]} \mathrm{dQ} Q_{[2,5,0],[2,3,4]}-\mathrm{dQ}_{[3,0],[3,4]} \mathrm{dQ} Q_{[2,5,0],[2,7,0]}\right) A_{2,1} A_{7,8} A_{2,2} \\
& +\left(d Q_{[3,0),(2,7]} d Q_{[2,3,0],(1,3,4]} d Q_{[5,0],(7,0)}-d Q_{[3,0],(1,7]} d Q_{[5,0],(3,4]} d Q_{[2,5,0],[2,7,8]}\right. \\
& -\mathrm{dQ}_{[3,0],[7,8]} \mathrm{d} \mathrm{Q}_{[3,8],(3,4]} \mathrm{d} \mathrm{Q}_{[2,8,0],(1,2,7])} A_{1,2} A_{2,1} A_{7,8}
\end{aligned}
$$

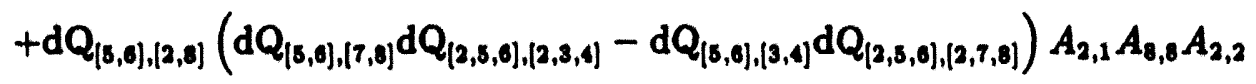

$$
\begin{aligned}
& +d Q_{[3,0],[2,8]}\left(-d Q_{[3,0],\{3,4]} d Q_{[2,5,0],[1,7,8]}+d Q_{[2,5,0],[1,3,4]} d Q_{[3,0],[7,8]}\right) A_{1,2} A_{2,1} A_{8,8}
\end{aligned}
$$

3.2.1.4.2 Simplifying cubic by adding Graßmann identities Graßmann-Plücker relations allow us to simplify one of the coefficients in 3.64 . Note that

$$
\begin{aligned}
& d Q_{[5,0],(7,8)} d Q_{[3,0],(3,4]} d Q_{[1,5,0],(1,2,3]}-d Q_{[3,0),(2,3]} d Q_{[1,5,0],(1,3,4]} d Q_{[3,0],(7,8]}+ \\
& \mathrm{dQ}_{[3,0],[1,3]} \mathrm{d} \mathrm{Q}_{[5,0],(3,4]} \mathrm{d} \mathrm{Q}_{[1,5,0],[2,7,8]}= \\
& d Q_{[3,0],[7,0]}\left(d Q_{[8,0],[3,4]} d Q_{[1,8,0],(1,2,3]}-d Q_{[5,0],[2,3]} d Q_{[1,5,0],[1,3,4]}\right)+ \\
& d Q_{[3,0],\{1,3]} d Q_{[3,0],(3,4]} d Q_{[1,5,0),(2,7,8]}
\end{aligned}
$$

If we consider the element of $G(3,7)$,

$$
\left[\begin{array}{lllllll}
Q_{1,1} & Q_{1,2} & Q_{1,3} & Q_{1,4} & 1 & 0 & 0 \\
Q_{3,1} & Q_{3,2} & Q_{3,3} & Q_{3,4} & 0 & 1 & 0 \\
Q_{0,1} & Q_{0,2} & Q_{0,3} & Q_{0,4} & 0 & 0 & 1
\end{array}\right]
$$

then we can write

$$
\text { (3.74) } d Q_{[5,6],\{3,4]} d Q_{[1,5,6],(1,2,3]}-d Q_{[5,6],(2,3]} d Q_{[1,5,6],(1,3,4]}=\pi_{3,4,5} \pi_{1,2,3}-\pi_{2,3,5} \pi_{1,3,4}
$$

but if we use the Graßmann relation 
47

$$
\pi_{3,4,8} \pi_{1,2,3}-\pi_{3,4,1} \pi_{8,2,3}+\pi_{3,4,2} \pi_{8,1,3}-\pi_{3,4,3} \pi_{3,1,2}=0
$$

we can simplify the right hand side of equation 3.72

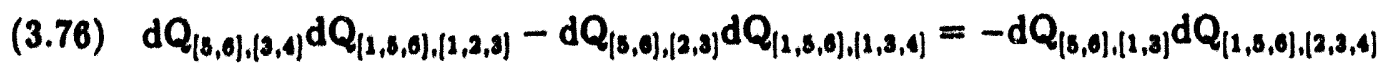

when we substitute this into the only "complicated" coefficient in 3.64 we get

$$
\begin{aligned}
& d Q_{[3,0],(7,8)} d Q_{[3,0),(3,4)} d Q_{[1,3,0],(1,2,3]}-d Q_{[3,0],(2,3]} d Q_{[1,3,0],(1,3,4]} d Q_{(3,0],(7,8)}+ \\
& \mathrm{dQ}_{[3,0],(1,3]} \mathrm{d} \mathrm{Q}_{[3,0],(3,4]} \mathrm{d} \mathrm{Q}_{[1,3,0],(2,7,8]}= \\
& -d Q_{[s, 0],(1,3]}\left(d Q_{[5,0),(7,0]} d Q_{[1,5,0),(2,3,4]}-d Q_{[1,5,0],(2,7,0]} d Q_{[5,0],(3,0)}\right)
\end{aligned}
$$

when we substitute this into equation 3.64 we get

$$
\begin{aligned}
& -A_{2,1} \mathrm{~d} Q_{[3,0),(1,2]} \mathrm{d} Q_{[8,0],[7,0]} \mathrm{d} Q_{[8,0],(3,4)} \\
& -\mathrm{d} Q_{[3,0],(2,3)}\left(\mathrm{d} Q_{[1,5,0),(1,3,4]} \mathrm{d} Q_{[3,0],[7,8]}-\mathrm{dQ}_{[1,5,0],(1,7,0]} \mathrm{d} Q_{[3,0],(3,4]}\right) A_{3,8} A_{2,2} A_{1,1}
\end{aligned}
$$

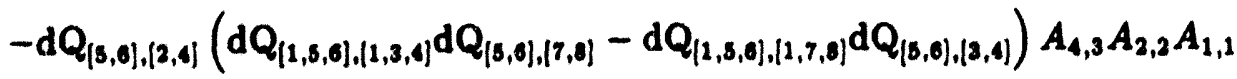

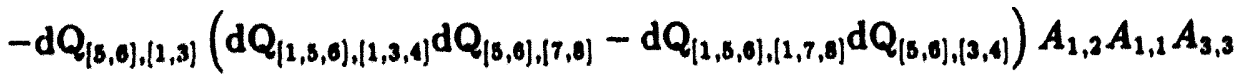

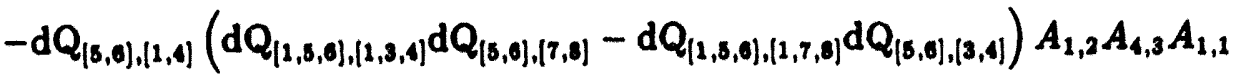

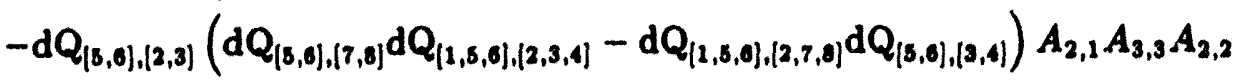

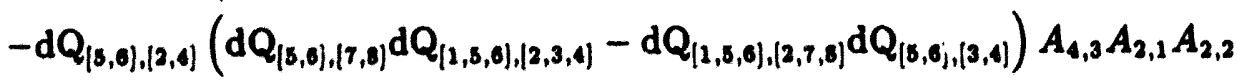

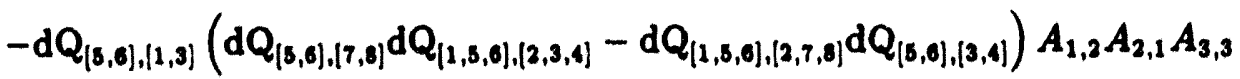

$$
\begin{aligned}
& -\mathrm{d} Q_{[3,8],(1,4]}\left(\mathrm{d} Q_{[3,0],[7,8]} \mathrm{d} Q_{[1,5,0],(2,3,4]}-\mathrm{d} Q_{[1,5,0],(2,7,8]} \mathrm{d} Q_{[3,0],(3,4)}\right) A_{1,2} A_{4,3} A_{2,1}
\end{aligned}
$$

And this simplified cubic equation factors neatly:

$$
\begin{aligned}
& -\left(\mathrm{dQ}_{[5,6],[2,3]} A_{3,3} A_{2,2}+\mathrm{dQ}_{[3,0],[2,4]} A_{4,3} A_{2,2}+\right. \\
& \left.\mathrm{dQ}_{[3,0],(1,3]} A_{1,2} A_{3,3}+\mathrm{d} Q_{[5,0],(1,4]} A_{1,2} A_{1,3}\right)
\end{aligned}
$$

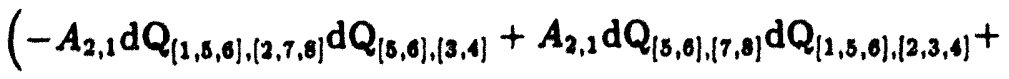

$$
\begin{aligned}
& \left.A_{1,1} \mathrm{dQ}_{[1,3,6],[1,3,4]} \mathrm{dQ}_{[5,0],\{7,8]}-A_{1,1} \mathrm{dQ}_{[1,5,0],\{1,7,8]} \mathrm{dQ}_{[8,6],(3,4]}\right)- \\
& A_{2,1} \mathrm{dQ}_{[(5,6),(1,2]} \mathrm{dQ} \mathrm{Q}_{[5,0],\{7,8]} \mathrm{dQ} \mathrm{Q}_{[5,0],(3,4]}
\end{aligned}
$$


After simplification with Grabmann-Plücker relations the rest of the cubic equations factor similarly:

$$
\begin{aligned}
& \left(\mathrm{d} \mathrm{Q}_{[1,2],(0,8]} A_{6,0} A_{8,7}+\mathrm{dQ} \mathrm{Q}_{[1,2],(0,7)} A_{7,7} A_{6,0}+\right. \\
& \left.\mathrm{dQ}_{[1,2],[3,8]} A_{8,8} A_{8,7}+\mathrm{dQ}_{[1,2],(8,7]} A_{8,0} A_{7,7}\right) \\
& \left(A_{8,8} \mathrm{~d} Q_{[8,6,8],(1,2,8]} \mathrm{d} Q_{[1,2],(5,0]}-A_{8,8} \mathrm{~d} Q_{[8,0],(1,2]} \mathrm{dQ}_{[1,2,8],(3,0,8]}\right. \\
& \left.A_{7,8} \mathrm{dQ}_{[8,0],\{1,2]} \mathrm{dQ}_{[1,2,8],(3,6,7]}+A_{7,8} \mathrm{~d} Q_{[1,2],[5,0]} \mathrm{dQ}_{[3,0,8],(1,2,7]}\right)- \\
& A_{7,8} \mathrm{dQ}_{[1,2],[7,8]} \mathrm{d} Q_{[1,2],(3,0]} \mathrm{d} Q_{[3,0],(1,2]} \\
& \left(A_{2,1} A_{8,8} \mathrm{dQ} Q_{[8,0],[2,8]}+\mathrm{dQ} Q_{[8,0],(1,7]} A_{1,1} A_{7,8}+\right. \\
& \left.A_{7,8} A_{2,1} \mathrm{dQ}_{[3,0],[2,7]}+A_{1,1} A_{8,8} \mathrm{dQ}_{[5,0],(1,8]}\right) \\
& \left(A_{8,7} \mathrm{dQ}_{[1,2],(8,0]} \mathrm{d} \mathrm{Q}_{[3,0,7],(1,2,8]}-A_{8,7} \mathrm{dQ}_{[5,0],(1,2]} \mathrm{dQ}_{[1,2,7],(3,0,8]}+\right. \\
& \left.A_{7,7} \mathrm{dQ}_{[1,2],(5,0]} \mathrm{dQ}_{[5,6,7],(1,2,7]}-\mathrm{dQ}_{[5,0],(1,2]} \mathrm{dQ}_{[1,2,7],(5,6,7]} A_{7,7}\right)- \\
& A_{8,7} \mathrm{dQ}_{[3,0],[7,8]} \mathrm{d} Q_{[5,0],(1,2]} \mathrm{d} Q_{[1,2],[5,6]} \\
& \left(\mathrm{dQ}_{[1,2],(6,8]} A_{6,6} A_{8,7}+\mathrm{dQ} \mathrm{Q}_{[1,2],[6,7]} A_{7,7} A_{6,6}+\right. \\
& \left.\mathrm{dQ}_{[1,2],[5,8]} A_{5,0} A_{8,7}+\mathrm{dQ} Q_{[1,2],[3,7]} A_{5,6} A_{7,7}\right) \\
& \left(A_{6,5} \mathrm{~d} Q_{[1,2],[3,4]} \mathrm{d} Q_{[1,2,5],[6,7,8]}-\mathrm{dQ}_{[1,2],[7,8]} \mathrm{d} Q_{[1,2,5],(3,4,0]} A_{6,5}+\right. \\
& \left.A_{5,3} \mathrm{dQ}_{[1,2],[3,4]} \mathrm{dQ} \mathrm{Q}_{[1,2,3],(5,7,8]}-A_{5,5} \mathrm{dQ}_{[1,2],[7,8]} \mathrm{dQ} \mathrm{Q}_{[1,2,5],(3,4,5]}\right)+ \\
& A_{6,5} \mathrm{dQ}_{[1,2],(5,0)} \mathrm{dQ}_{[1,2],(3,4]} \mathrm{dQ}_{[1,2],(7,8)} \\
& -\left(-A_{1,2} \mathrm{~d} Q_{[2,5,6],(1,3,4]} \mathrm{d} Q_{[5,0],[7,8]}+A_{1,2} \mathrm{dQ}_{[5,0],(3,4]} \mathrm{d} Q_{[2,5,0],(1,7,8]}-\right. \\
& \left.A_{2,2} \mathrm{dQ}_{[3,0],[7,8]} \mathrm{dQ}_{[2,5,0],[2,3,4]}+A_{2,2} \mathrm{dQ}_{[3,0],[3,4]} \mathrm{dQ}_{[2,5,6],[2,7,8]}\right) \\
& \left(A_{2,1} A_{8,8} \mathrm{dQ}_{[5,0],[2,8]}+\mathrm{dQ}_{[3,0],(1,7]} A_{1,1} A_{7,8}+A_{7,8} A_{2,1} \mathrm{dQ}_{[5,0],[2,7]}+\right. \\
& \left.A_{1,1} A_{8,8} \mathrm{~d} Q_{[5,6],(1,8]}\right)+A_{1,2} \mathrm{dQ}_{[5,6],(1,2]} \mathrm{dQ}_{[5,6],[7,8]} \mathrm{d} \mathrm{Q}_{[5,0],[3,4]}
\end{aligned}
$$




$$
\begin{aligned}
& \left(\mathrm{dQ}_{[3,0],[2,3]} A_{3,3} A_{2,2}+\mathrm{dQ}\left[(3,0],\{2,4] A_{4,3} A_{2,2}+\right.\right. \\
& \left.\mathrm{dQ}_{[3,0],(1,3]} A_{1,2} A_{3,3}+\mathrm{dQ}_{[3,0],(1,4]} A_{1,2} A_{4,3}\right) \\
& \left(-A_{4,4} \mathrm{dQ}_{[5,0],[1,2]} \mathrm{d} \mathrm{Q}_{[1,2,4],[4,5,0]}-A_{3,4} \mathrm{dQ}_{[8,0],[1,2]} \mathrm{dQ}_{[1,2,0],(3,5,0]}+\right. \\
& \left.A_{3,4} \mathrm{dQ}_{[1,2],[3,0]} \mathrm{dQ}_{[4,5,0],(1,2,3]}+A_{4,4} \mathrm{dQ}_{[1,2],(3,0]} \mathrm{dQ} \mathrm{Q}_{[4,8,0],(1,2,4]}\right)+ \\
& A_{3,4} \mathrm{dQ}_{[3,0],(3,4)} \mathrm{dQ}_{[1,2],(3,0]} \mathrm{dQ}_{[5,0],[1,2]} \\
& -\left(A_{0,5} A_{3,4} \mathrm{dQ} Q_{[1,2],[3,0]}+\mathrm{dQ}_{[1,2],\{3,5]} A_{5,5} A_{3,4}+\right. \\
& \left.\mathrm{dQ}_{[1,2],[4,5]} A_{5,5} A_{4,4}+\mathrm{dQ}_{[1,2],[4,0]} A_{6,5} A_{6,4}\right) \\
& \left(\mathrm{dQ}_{[1,2],[5,0]} \mathrm{d} Q_{[3,5,0],[1,2,4]} A_{4,3}-\mathrm{dQ}_{[1,2,3],[4,5,0]} \mathrm{d} Q_{[5,0],[1,2]} A_{4,3}-\right. \\
& \left.A_{3,3} \mathrm{dQ}_{[5,0],[1,2]} \mathrm{dQ}_{[1,2,3],(3,5,6]}+A_{3,3} \mathrm{dQ}_{[1,2],[5,0]} \mathrm{dQ}_{[3,5,0],(1,2,3]}\right)+ \\
& A_{4,3} \mathrm{dQ}_{[1,2],(3,4]} \mathrm{dQ}_{[1,2],(5,0]} \mathrm{dQ}_{[5,0],[1,2]} \\
& \left(A_{3,0} \mathrm{dQ} Q_{[1,2],(3,4]} \mathrm{dQ}_{[1,2,6],[3,7,8]}-A_{6,6} \mathrm{~d} Q_{[1,2,0],[3,4,6]} \mathrm{dQ}_{[1,2],[7,8]}+\right. \\
& \left.A_{6,6} \mathrm{dQ}_{[1,2],[3,4]} \mathrm{dQ}_{[1,2,0),(6,7,8]}-A_{5,6} \mathrm{dQ}_{[1,2,6],(3,4,5]} \mathrm{dQ}_{[1,2],(7,8]}\right) \\
& \left(A_{6,5} A_{3,4} \mathrm{dQ}_{[1,2],[3,0]}+\mathrm{dQ}_{[1,2],[3,5]} A_{5,5} A_{3,4}+\right. \\
& \left.\mathrm{dQ}_{[1,2],(4,5]} A_{5,5} A_{4,4}+\mathrm{dQ}_{[1,2],[4,0]} A_{6,5} A_{4,4}\right)-
\end{aligned}
$$

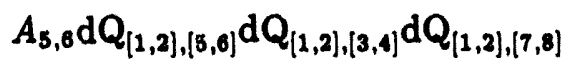

3.2.1.4.3 Solving the Equations The variables in these equations appear in a very systematic form. Each equation is a polynomial in six variables: four from one of the $2 \times 2$ subblocks of $A$, the other two from one of the neighboring subblocks of $A$. Furthermore, for each cubic there exists a quadratic which is a function of the same six $A_{i, j}$ 's. Finally, each of the equations is linear in the $A_{i, j}$ 's from the neighboring subblock. The following table shows the pairings of variables of the quadratics and the cubics: 


$\begin{array}{cccc}\begin{array}{c}\text { equation } \\ \text { pair }\end{array} & \text { linear variable } & \begin{array}{c}\text { pair of two } \\ \text { linear variables }\end{array} \\ 1 & A_{2,1} & A_{3,3}, A_{4,3} & A_{1,1}, A_{1,2}, A_{2,1}, A_{2,2} \\ 2 & A_{7,8} & A_{6,6}, A_{7,6} & A_{7,7}, A_{7,8}, A_{8,7}, A_{8,8} \\ 3 & A_{8,7} & A_{1,1}, A_{2,1} & A_{7,7}, A_{7,8}, A_{8,7}, A_{8,8} \\ 4 & A_{6,5} & A_{7,7}, A_{8,7} & A_{5,5}, A_{5,6}, A_{6,5}, A_{6,6} \\ 5 & A_{1,2} & A_{7,8}, A_{8,8} & A_{1,1}, A_{1,2}, A_{2,1}, A_{2,2} \\ 6 & A_{3,4} & A_{1,2}, A_{2,2} & A_{3,3}, A_{3,4}, A_{4,3}, A_{4,4} \\ 7 & A_{4,3} & A_{5,5}, A_{6,5} & A_{3,3}, A_{3,4}, A_{4,3}, A_{4,4} \\ 8 & A_{5,6} & A_{3,4}, A_{4,4} & A_{5,5}, A_{5,6}, A_{6,5}, A_{6,6}\end{array}$

(Note that the first column applies only to the cubics.)

From the above chart, we can see that we may either solve pairs 2,3,6, and 7 for the subblocks of $A$ containing $A_{1,1}, A_{1,2}, A_{2,1}, A_{2,2}, A_{5,5}, A_{5,6}, A_{6,5}$, and $A_{6,6}$, (or vice-versa). After making these linear solves we get solutions of the form 
51

$$
\begin{aligned}
& A_{1,2}=\mathrm{dQ}_{[5,6],[3,4]}\left(-\mathrm{dQ}_{[1,2],[5,6]}^{2} \mathrm{dQ}_{[5,6],[1,2]}^{2}\left(\mathrm{dQ}_{[5,6],[2,3]} A_{3,4}^{2}+\mathrm{dQ}_{[5,6],[2,4]} A_{4,4} A_{3,4}\right)\right. \\
& +\mathrm{d} \mathrm{Q}_{[5,6],[2,3]}\left(\mathrm{dQ}_{[1,2,4],[3,5,0]} \mathrm{d} \mathrm{Q}_{[5,6],[1,2]}-\mathrm{d} \mathrm{Q}_{[1,2],[5,6]} \mathrm{d} \mathrm{Q}_{[4,5,6],[1,2,3]}\right)^{2} A_{3,4}^{2} A_{3,3}^{2} \\
& +\left(d Q_{[5,6],[2,3]} d Q_{[1,2],[5,6]}^{2} d Q_{[1,5,6],[1,2,4]}^{2}\right. \\
& +2 d Q_{[5,6],[2,4]} d Q_{[5,6],[1,2]}^{2} d Q_{[1,2,4],[4,5,6]} d Q_{[1,2,4],[3,5,6]} \\
& -d Q_{[5,6],[2,3]} d Q_{[1,2],[5,6]} d Q_{[4,5,6],[1,2,4]} d Q_{[5,6],[1,2]} d Q_{[1,2,4],[4,5,6]} \\
& -d Q_{[5,6],[2,4]} d Q_{[5,6],[1,2]} d Q_{[1,2,4],[3,5,6]} d Q_{[1,2],[5,6]} d Q_{[4,5,6],[1,2,4]} \\
& +d Q_{[5,6],[2,4]} d Q_{[1,2],[5,6]}^{2} d Q_{[4,5,6],[1,2,3]} d Q_{[4,5,6],[1,2,4]} \\
& \left.-2 \mathrm{dQ}_{[5,6],[2,4]} \mathrm{d} \mathrm{Q}_{[5,6],[1,2]} \mathrm{d} \mathrm{Q}_{[1,2,4],[4,5,6]} \mathrm{d} Q_{[1,2],[5,6]} \mathrm{d} \mathrm{Q}_{[4,5,6],[1,2,3]}\right) A_{3,4} A_{4,3} A_{4,4} A_{3,3} \\
& +\left(d Q_{[5,6],[1,2]} d Q_{[1,2,4],[4,5,6]}-d Q_{[1,2],[5,6]} d Q_{[4,5,6],[1,2,4]}\right) \\
& \mathrm{dQ}_{[5,6],[1,2]} \mathrm{dQ}_{[5,6],[2,3]} \mathrm{dQ}_{[1,2,4],\{4,5,6]} A_{4,4}^{2} A_{3,3}^{2} \\
& +d Q_{[5,6],[2,3]}\left(-d Q_{[1,2],[5,6]} d Q_{[4,5,6],[1,2,4]}+2 d Q_{[5,6],[1,2]} d Q_{[1,2,4],[4,5,6]}\right) \\
& \left(\mathrm{dQ}_{[1,2,4],[3,5,6]} \mathrm{d} \mathrm{Q}_{[5,6],[1,2]}-\mathrm{dQ}_{[1,2],[5,6]} \mathrm{d} \mathrm{Q}_{[4,5,6],[1,2,3]}\right) A_{3,4} A_{4,4} A_{3,3}^{2} \\
& -\left(d Q_{[5,6],[1,2]} d Q_{[1,2,4],[4,5,6]}-d Q_{[1,2],[5,6]} d Q_{[4,5,6],[1,2,4]}\right) \\
& \mathrm{dQ}_{[5,6],\{2,4]} \mathrm{d} Q_{[1,2],[5,6]} \mathrm{dQ}_{[4,5,6],[1,2,4]} A_{3,4} A_{4,4} A_{4,3}^{2} \\
& +\left(\mathrm{dQ}_{[5,6],[1,2]} \mathrm{d} \mathrm{Q}_{[1,2,4],[4,5,6]}-\mathrm{d} \mathrm{Q}_{[1,2],[5,6]} \mathrm{d} \mathrm{Q}_{[4,5,6],[1,2,4]}\right) \\
& \mathrm{dQ}_{[5,6],[1,2]} \mathrm{dQ}_{[5,6],[2,4]} \mathrm{dQ}_{[1,2,4],[4,5,6]} A_{3,3} A_{4,4}^{2} A_{4,3} \\
& -\left(d Q_{[1,2,4],\{3,5,6]} d Q_{[5,6],[1,2]}-d Q_{[1,2],[5,6]} d Q_{[4,5,6],[1,2,3]}\right) \\
& \mathrm{dQ}_{[5,6],[2,4]} \mathrm{dQ} \mathrm{Q}_{[1,2],[5,8]} \mathrm{d} \mathrm{Q}_{[4,5,6],[1,2,4]} A_{3,4}^{2} A_{4,3}^{2} \\
& +\left(\mathrm{dQ}_{[1,2,4],[3,5,6]} \mathrm{d} \mathrm{Q}_{[5,6],[1,2]}-\mathrm{d} \mathrm{Q}_{[1,2],[5,6]} \mathrm{d} \mathrm{Q}_{[4,5,6],[1,2,3]}\right) \\
& \left(d Q_{[5,6],[2,4]} d Q_{[1,2,4],[3,5,6]} d Q_{[5,6],[1,2]}-d Q_{[5,6],[2,4]} d Q_{[1,2],(5,6]} d Q_{[4,5,6],[1,2,3]}-\right. \\
& \left.\mathrm{dQ}_{[5,6],[2,3]} \mathrm{dQ}_{[1,2],[5,6]} \mathrm{dQ}_{[4,5,6],[1,2,4]}\right) A_{3,3} A_{3,4}^{2} A_{4,3} / \\
& \mathrm{dQ}_{[1,2],[5,6]} \mathrm{dQ}_{[5,6],[1,2]} d A_{[3,4],[3,4]}\left(A_{4,4} \mathrm{dQ}_{[5,6],[1,2]} \mathrm{dQ}_{[1,2,4],[4,5,6]}\right. \\
& -A_{4,4} \mathrm{dQ}_{[1,2],[5,6]} \mathrm{dQ}_{[4,5,6],[1,2,4]}+ \\
& \left.A_{3,4} \mathrm{dQ}_{[5,6],[1,2]} \mathrm{dQ}_{[1,2,4],[3,5,6]}-A_{3,4} \mathrm{dQ}_{[1,2],[5,6]} \mathrm{dQ}_{[4,5,6],[1,2,3]}\right) \\
& \left(-d Q_{[5,6],[2,4]} d Q_{[5,6],[1,3]}+d Q_{[5,6],[1,4]} d Q_{[5,6],[2,3]}\right)
\end{aligned}
$$

The denominator may be simplified (a little) with the Graßmann relation

$$
-d Q_{[5,6],[2,3]} d Q_{[5,6],[1,4]}+d Q_{[5,6],[2,4]} d Q_{[5,6],[1,3]}=d Q_{[5,6],[1,2]} d Q_{[5,6],[3,4]}
$$


52

The result is

$$
\begin{aligned}
& A_{1,2}=\left(-\mathrm{dQ}_{[1,2],[5,6]}^{2} \mathrm{dQ}_{[5,6],[1,2]}^{2}\left(\mathrm{dQ}_{[5,6],[2,3]} A_{3,4}^{2}+\mathrm{dQ}_{[5,6],[2,4]} A_{4,4} A_{3,4}\right)\right. \\
& +\mathrm{dQ}_{[5,6],[2,3]}\left(\mathrm{dQ}_{[1,2,4],[3,5,6]} \mathrm{d} \mathrm{Q}_{[5,6],[1,2]}-\mathrm{d} \mathrm{Q}_{[1,2],[5,6]} \mathrm{d} \mathrm{Q}_{[4,5,6],[1,2,3]}\right)^{2} A_{3,4}^{2} A_{3,3}^{2} \\
& +\left(d Q_{[5,6],[2,3]} d Q_{[1,2],[5,6]}^{2} d Q_{[4,5,6],[1,2,4]}^{2}\right. \\
& +2 d Q_{[5,6],[2,4]} d Q_{[5,6],[1,2]}^{2} d Q_{[1,2,4],[4,5,6]} d Q_{[1,2,4],[3,5,6]} \\
& -d Q_{[5,6],[2,3]} d Q_{[1,2],[5,6]} d Q_{[4,5,6],[1,2,4]} d Q_{[5,6],[1,2]} d Q_{[1,2,4],[4,5,6]} \\
& -d Q_{[5,6],[2,4]} d Q_{[5,6],[1,2]} d Q_{[1,2,4],[3,5,6]} d Q_{[1,2],[5,6]} d Q_{[4,5,6],[1,2,4]} \\
& +d Q_{[5,6],[2,4]} d Q_{[1,2],[5,6]}^{2} d Q_{[4,5,6],[1,2,3]} d Q_{[4,5,6],\{1,2,4]} \\
& \left.-2 \mathrm{dQ}_{[5,6],[2,4]} \mathrm{d} \mathrm{Q}_{[5,6],[1,2]} \mathrm{dQ}_{[1,2,4],[4,5,6]} \mathrm{d} \mathrm{Q}_{[1,2],[5,6]} \mathrm{dQ} \mathrm{Q}_{[4,5,6],[1,2,3]}\right) A_{3,4} A_{4,3} A_{4,4} A_{3,3} \\
& +\left(d Q_{[5,6],[1,2]} d Q_{[1,2,4],[4,5,6]}-d Q_{[1,2],[5,6]} d Q_{[4,5,6],[1,2,4]}\right) \\
& \mathrm{dQ}_{[5,6],[1,2]} \mathrm{dQ} \mathrm{Q}_{[5,6],[2,3]} \mathrm{dQ}_{[1,2,4],[4,5,6]} A_{4,4}^{2} A_{3,3}^{2} \\
& +d Q_{[5,6],[2,3]}\left(-d Q_{[1,2],[5,6]} d Q_{[4,5,6],[1,2,4]}+2 d Q_{[5,6],[1,2]} d Q_{[1,2,4],[4,5,6]}\right) \\
& \left(\mathrm{dQ}_{[1,2,4],[3,5,6]} \mathrm{dQ} Q_{[5,6],[1,2]}-\mathrm{dQ}_{[1,2],[5,6]} \mathrm{dQ}_{[4,5,6],[1,2,3]}\right) A_{3,4} A_{4,4} A_{3,3}^{2} \\
& -\left(d Q_{[5,6],[1,2]} d Q_{[1,2,4],[4,5,6]}-d Q_{[1,2],[5,6]} d Q_{[4,5,6],[1,2,4]}\right) \\
& \mathrm{dQ}_{[5,6],[2,4]} \mathrm{dQ} \mathrm{Q}_{[1,2],[5,6]} \mathrm{dQ}_{[4,5,6],[1,2,4]} A_{3,4} A_{4,4} A_{4,3}^{2} \\
& +\left(\mathrm{dQ}_{[5,6],[1,2]} \mathrm{d} \mathrm{Q}_{[1,2,4],[4,5,6]}-\mathrm{d} \mathrm{Q}_{[1,2],[5,6]} \mathrm{d} \mathrm{Q}_{[4,5,6],[1,2,4]}\right) \\
& \mathrm{dQ}_{[5,6],[1,2]} \mathrm{dQ}_{[5,6],[2,4]} \mathrm{dQ}_{[1,2,4],[4,5,6]} A_{3,3} A_{4,4}^{2} A_{4,3} \\
& -\left(d Q_{[1,2,4],[3,5,6]} d Q_{[5,6],[1,2]}-d Q_{[1,2],[5,6]} d Q_{[4,5,6],[1,2,3]}\right) \\
& \mathrm{dQ}_{[5,6],[2,4]} \mathrm{d} Q_{[1,2],[5,6]} \mathrm{dQ} Q_{[4,5,6],[1,2,4]} A_{3,4}^{2} A_{4,3}^{2} \\
& +\left(d Q_{[1,2,4],[3,5,6]} d Q_{[5,6],[1,2]}-d Q_{[1,2],[5,6]} d Q_{[4,5,6],[1,2,3]}\right) \\
& \left(d Q_{[5,6],[2,4]} d Q_{[1,2,4],(3,5,6]} d Q_{[5,6],(1,2]}-d Q_{[5,6],\{2,4]} d Q_{[1,2],[5,6]} d Q_{[4,5,6],(1,2,3]}-\right. \\
& \left.\mathrm{dQ}_{[5,6],[2,3]} \mathrm{dQ} \mathrm{Q}_{[1,2],[5,6]} \mathrm{dQ} \mathrm{Q}_{[4,5,6],[1,2,4]}\right) A_{3,3} A_{3,4}^{2} A_{4,3} / \\
& \mathrm{dQ}_{[1,2],[5,6]} \mathrm{dQ}_{[5,6],[1,2]} d A_{[3,4],[3,4]}\left(A_{4,4} \mathrm{dQ}_{[5,6],\{1,2]} \mathrm{dQ}_{[1,2,4],[4,5,6]}\right. \\
& -A_{4,4} \mathrm{dQ}_{[1,2],[5,6]} \mathrm{d} \mathrm{Q}_{[4,5,6],[1,2,4]}+ \\
& \left.A_{3,4} \mathrm{dQ}_{[5,6],[1,2]} \mathrm{dQ} \mathrm{Q}_{[1,2,4],[3,5,6]}-A_{3,4} \mathrm{dQ}_{[1,2],[5,6]} \mathrm{d} \mathrm{Q}_{[4,5,6],[1,2,3]}\right) \mathrm{d} \mathrm{Q}_{[5,6],[1,2]}
\end{aligned}
$$

Once solutions of this form are substituted into equation pairs $1,4,5$ and 8 , we are left with eight messy equations. The equations which were once cubic now have twenty terms; the equations which were once quadratic have one hundred terms. All eight equations 
53

share one important feature: the remaining $A_{i, j}$ 's appear in pairs. To solve the last eight equations we make the substitutions

(3.88) . $\quad A_{4,3}=A_{3,3} q_{1}, A_{4,4}=A_{3,4} q_{2}, A_{8,7}=A_{7,7} q_{3}, A_{8,8}=A_{7,8} q_{4}$

One of the four (previously) cubic equations is shown below.

$$
\begin{aligned}
& -q_{1} A_{3,4}^{2}\left(-\mathrm{dQ}_{[3,5,0],[1,2,4]} \mathrm{dQ}_{[1,2],[3,0]}+\mathrm{dQ}_{[1,2,3],[4,5,0]} \mathrm{d} \mathrm{Q}_{[5,0],[1,2]}\right) \\
& \left(\left(-d Q_{[5,6,8],[1,2,7]} d Q_{[1,2],[4,5]} d Q_{[1,2],(5,6]}+d Q_{[5,6],[1,2]} d Q_{[1,2],[4,5]} d Q_{[1,2,8],[5,6,7]}+\right.\right. \\
& \left.d Q_{[1,2,5],[4,7,8]} d Q_{[5,6],\{1,2]} d Q_{[1,2],[5,8]}\right) \\
& \left(d Q_{[5,6],[1,2]} d Q_{[1,2,3],[3,5,6]}-2 d Q_{[3,5,6],[1,2,3]} d Q_{[1,2],[5,6]}\right) q_{2}{ }^{2} \\
& +\left(d Q_{[5,6],[1,2]}^{2} d Q_{[1,2,3],[4,5,6]} d Q_{[1,2],[3,5]} d Q_{[1,2,8],(5,6,7]}+\right. \\
& d Q_{[5,6],[1,2]}^{2} d Q_{[1,2],[4,5]} d Q_{[1,2,3],[3,5,6]} d Q_{[1,2,8],(5,6,7]} \\
& +d Q_{[5,6],\{1,2]}^{2} d Q_{[1,2,3],(3,5,6]} d Q_{[1,2],[5,6]} d Q_{[1,2,5],[4,7,8]} \\
& -d Q_{[1,2],[5,6]} d Q_{[5,6],\{1,2]} d Q_{[1,2],[4,5]} d Q_{[1,2,3],\{3,5,6]} d Q_{[5,6,8],[1,2,7]} \\
& -d Q_{[1,2],[5,6]} d Q_{[5,6],[1,2]} d Q_{[1,2],[3,5]} d Q_{[3,5,6],[1,2,4]} d Q_{[1,2,8],\{5,6,7]} \\
& -d Q_{[1,2],[5,6]} d Q_{[5,6],[1,2]} d Q_{[1,2],[3,5]} d Q_{[5,6,8],[1,2,7]} d Q_{[1,2,3],\{4,5,6]} \\
& +d Q_{[1,2],\{5,6]}^{2} d Q_{[1,2],(3,5]} d Q_{[3,5,6],[1,2,4]} d Q_{[5,6,8],[1,2,7]} \\
& -d Q_{[1,2],\{5,6]}^{2} d Q_{[5,6],(1,2]} d Q_{[3,5,6],[1,2,4]} d Q_{[1,2,5],[3,7,8]} \\
& \left.+\mathrm{dQ}_{[5,6],(1,2]}^{2} \mathrm{~d} \mathrm{Q}_{[1,2,3],[4,5,6]} \mathrm{d} \mathrm{Q}_{[1,2],(5,8)} \mathrm{d} \mathrm{Q}_{[1,2,5],(3,7,8]}\right) q_{1} q_{2}
\end{aligned}
$$

$$
\begin{aligned}
& +d Q_{[5,6],(1,2]} d Q_{[1,2],[3,5]} d Q_{[1,2,3],[3,5,6]} \\
& \quad\left(d Q_{[1,2,8],[5,6,8]} d Q_{[5,6],[1,2]}-d Q_{[5,6,8],[1,2,8]} d Q_{[1,2],[5,6]}\right) q_{4} q_{1} \\
& +d Q_{[1,2],[4,5]}\left(d Q_{[1,2,8],[5,6,8]} d Q_{[5,6],[1,2]}-d Q_{[5,6,8],[1,2,8]} d Q_{[1,2],[5,6]}\right) \\
& \quad\left(d Q_{[5,6],[1,2]} d Q_{[1,2,3],[3,5,6]}-2 d Q_{[3,5,6],[1,2,3]} d Q_{[1,2],[5,6]}\right) q_{4} q_{2}{ }^{2} \\
& +\left(d Q_{[1,2,8],[5,6,8]} d Q_{[5,6],[1,2]}-d Q_{[5,6,8],[1,2,8]} d Q_{[1,2],[5,6]}\right) \\
& \quad\left(d Q_{[5,6],[1,2]} d Q_{[1,2],[3,5]} d Q_{[1,2,3],[4,5,6]}+d Q_{[5,6],[1,2]} d Q_{[1,2],[4,5]} d Q_{[1,2,3],[3,5,6]}\right. \\
& \left.\quad-d Q_{[1,2],[5,6]]} d Q_{[1,2],[3,5]} d Q_{[3,5,6],[1,2,4]}\right) q_{4} q_{2} q_{1} \\
& +d Q_{[5,6],[1,2]} d Q_{[1,2,3],[3,5,6]}\left(-d Q_{[5,6,8],[1,2,7]} d Q_{[1,2],[3,5]} d Q_{[1,2],[5,6]}\right. \\
& \left.\left.+d Q_{[5,6],[1,2]} d Q_{[1,2],[3,5]} d Q_{[1,2,8],[5,6,7]}+d Q_{[5,6],[1,2]} d Q_{[1,2],[5,6]} d Q_{[1,2,5],[3,7,8]}\right) q_{1}\right)
\end{aligned}
$$


54

$$
\begin{aligned}
& +q_{1}\left(\mathrm { d } _ { [ 5 , 0 ] , [ 1 , 2 ] } ^ { 2 } \mathrm { d } \mathrm { Q } _ { [ 1 , 2 ] , [ 5 , 0 ] } ^ { 2 } \left(-\mathrm{d} \mathrm{Q}_{[3,0,0],[1,2,7]} \mathrm{d} \mathrm{Q}_{[1,2],[3,5]} \mathrm{d} \mathrm{Q}_{[1,2],(3,0]}\right.\right. \\
& \left.+d Q_{[5,06],(1,2]} d Q_{[1,2],(3,8]} d Q_{[1,2,8],(3,6,7]}+d Q_{[5,0],\{1,2]} d Q_{[1,2],(3,0]} d Q_{[1,2,5],(3,7,8]}\right) \\
& +d Q_{[3,0],[1,2]}^{2} d Q_{[1,2],[4,5]} d Q_{[1,2],[5,0]}^{2} \\
& \left(\mathrm{dQ}_{[1,2,8],[5,6,8]} \mathrm{dQ} \mathrm{Q}_{[5,6],(1,2]}-\mathrm{d} \mathrm{Q}_{[5,0,8],[1,2,8]} \mathrm{d} \mathrm{Q}_{[1,2],[5,6]}\right) q_{4} q_{1} \\
& -\left(-d Q_{[3,5,6],[1,2,1]} d Q_{[1,2],[3,0]}+d Q_{[1,2,3],[4,5,0]} d Q_{[5,6],[1,2]}\right)^{2} \\
& \left(-d Q_{[5,6,8],[1,2,7]} d Q_{[1,2],[4,8]} d Q_{[1,2],[5,6]}+d Q_{[5,6],(1,2]} d Q_{[1,2],[4,5]} d Q_{[1,2,8],(5,6,7]}+\right.
\end{aligned}
$$

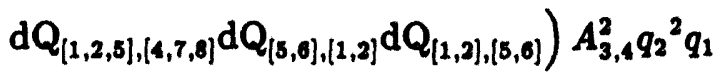

$$
\begin{aligned}
& -\left(-d Q_{[3,0],[1,2]}^{2} d Q_{[1,2,3],[3,5,0]} d Q_{[1,2],[5,6]}^{2} d Q_{[3,5,6],(1,2,4]} d Q_{[1,2,5],(3,7,8]}\right. \\
& -2 d Q_{[1,2],(5,6]} d Q_{[1,2],\{3,5]} d Q_{[1,2,3],\{4,5,6]} d Q_{[5,0],\{1,2]}^{2} d Q_{[3,5,0],[1,2,3]} d Q_{[1,2,8],\{5,6,7]} \\
& +d Q_{[1,2],[5,6]}^{2} d Q_{[1,2],[4,5]} d Q_{[3,5,6],(1,2,3]} d Q_{[1,2,3],(3,5,6]} d Q_{[5,6],(1,2]} d Q_{[5,6,8],(1,2,7]} \\
& +2 d Q_{[1,2],[5,6]}^{2} d Q_{[1,2],[3,5]} d Q_{[3,5,6],(2,2,4)} d Q_{[3,5,6],\{1,2,3]} d Q_{[5,6],\{1,2]} d Q_{[1,2,8],[5,6,7]} \\
& +d Q_{[1,2],[5,6]}^{2} d Q_{[1,2],[3,5]} d Q_{[3,6],(1,2]} d Q_{[1,2,3],[3,5,6]} d Q_{[3,5,0],(1,2,4]} d Q_{[5,6,8],(1,2,7]} \\
& +d Q_{[5,6],(1,2]}^{3} d Q_{[1,2],\{3,5]} d Q_{[1,2,3],(3,5,0]} d Q_{[1,2,3],\{1,5,8]} d Q_{[1,2,8],\{5,6,7]} \\
& -d Q_{[1,2],[5,6]} d Q_{[5,6],[1,2]}^{2} d Q_{[1,2],[3,5]} d Q_{[1,2,3],(3,5,6]} d Q_{[3,5,6],(1,2,4]} d Q_{[1,2,8],(5,6,7]} \\
& +2 d Q_{[1,2],[3,6]}^{2} d Q_{[1,2],(3,5]} d Q_{[1,2,3],\{4,5,6]} d Q_{[5,0],\{1,2]} d Q_{[3,5,6],(1,2,3]} d Q_{[5,6,8],\{1,2,7]} \\
& +d Q_{[1,2],(4,5]} d Q_{[5,6],\{1,2]}^{3} d Q_{[1,2,3],\{3,5,6]}^{2} d Q_{[1,2,8],(3,6,7]} \\
& -d Q_{[1,2],\{5,6]} d Q_{[1,2],\{1,5]} d Q_{[5,6],[1,2]}^{2} d Q_{[1,2,3],[3,5,6]}^{2} d Q_{[5,6,8],\{1,2,7]} \\
& -2 d Q_{[1,2],\{3,5]} d Q_{[3,5,6],\{1,2,4]} d Q_{[3,5,6],[1,2,3]} d Q_{[5,6,8],\{1,2,7]} d Q_{[1,2],[5,6]}^{3} \\
& -d Q_{[1,2],(5,6]} d Q_{[1,2],[4,5]} d Q_{[3,5,6],(1,2,3]} d Q_{[1,2,3],(3,5,6]} d Q_{[5,8],(1,2]}^{2} d Q_{[1,2,8],[5,6,7]} \\
& -d Q_{[1,2],[5,6]} d Q_{[5,6],\{1,2]}^{2} d Q_{[1,2],[3,5]} d Q_{[1,2,3],[3,5,6]} d Q_{[1,2,3],\{4,5,6]} d Q_{[5,6,8],[1,2,7]} \\
& +d Q_{[5,6],[1,2]}^{3} d Q_{[1,2,3],[3,5,6]} d Q_{[1,2,3],\{4,5,6]} d Q_{[1,2],\{5,6]} d Q_{[1,2,5],(3,7,8]} \\
& -2 d Q_{[1,2],[5,8]}^{2} d Q_{[5,6],[1,2]}^{2} d Q_{[3,5,8],[1,2,3]} d Q_{[1,2,3],\{1,5,6]} d Q_{[1,2,5],\{3,7,8]} \\
& -d Q_{[1,2],[5,6]}^{2} d Q_{[3,5,6],(1,2,3]} d Q_{[5,6],[1,2]}^{2} d Q_{[1,2,3],[3,5,6]} d Q_{[1,2,5],[4,7,8]} \\
& +d Q_{[5,6],[1,2]}^{3} d Q_{[1,2],(5,6)} d Q_{[1,2,3],[3,5,6]}^{2} d Q_{[1,2,5],\{4,7,8]} \\
& \left.+2 \mathrm{dQ}_{[5,6],\{1,2]} \mathrm{d} \mathrm{Q}_{[1,2],[5,6]}^{3} \mathrm{dQ} \mathrm{Q}_{[3,5,6],[1,2,3]} \mathrm{d} \mathrm{Q}_{[3,5,6],(1,2,4]} \mathrm{d} \mathrm{Q}_{[1,2,5],(3,7,8]}\right) q_{2} A_{3,4}^{2}
\end{aligned}
$$




$$
\begin{aligned}
& -\left(-d Q_{[3,5,0],[1,2,3]} d Q_{[1,2],(5,0]}+d Q_{[5,0],(1,2]} d Q_{[1,2,3],(3,5,0]}\right) A_{3,4}^{2} \\
& +\left(-d Q_{[3,5,0],(1,2,3]} d Q_{[2,2],[3,0]}\left(-d Q_{[3,0,0],(1,2,7]} d Q_{[1,2],(1,8)} d Q_{[1,2],(3,0]}\right.\right. \\
& \left.+d Q_{[5,0),(1,2]} d Q_{[1,2],(4,5]} d Q_{[1,2,8],(5,0,7]}+d Q_{[1,2,5],(4,7,8]} d Q_{[5,0],(1,2]} d Q_{[1,2],(8,0]}\right) q_{2}{ }^{2} \\
& +\left(d Q_{[1,2,8],(3,0,8]} d Q_{[5,0],(1,2]}-d Q_{[5,0,8],(1,2,8]} d Q_{[1,2],(8,0)}\right)
\end{aligned}
$$

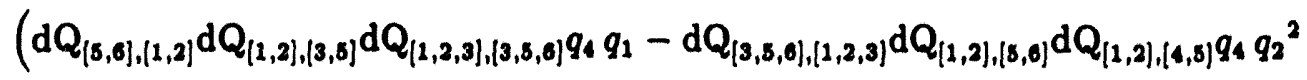

$$
\begin{aligned}
& \left.-\mathrm{dQ}_{[1,2],(3,5]} \mathrm{d} \mathrm{Q}_{[3,5,0),(1,2,3]} \mathrm{d} \mathrm{Q}_{[1,2],(5,0)} q_{4} q_{2}\right) \\
& +d Q_{[3,0],(1,2]} d Q_{[1,2,3],(3,5,0]}\left(-d Q_{[3,6,8],(1,2,7]} d Q_{[1,2],[3,5]} d Q_{[1,2],[5,0]}+\right. \\
& \left.d Q_{[5,8],(1,2]} d Q_{[1,2],(3,5]} d Q_{[1,2,8],(8,0,7]}+d Q_{[5,0],[1,2]} d Q_{[1,2],(8,8)} d Q_{[1,2,5],(3,7,8]}\right) q_{1} \\
& -d Q_{[3,5,6],[1,2,3]} d Q_{[1,2],[5,0]}\left(-d Q_{[5,6,8],[1,2,7]} d Q_{[1,2],[3,8]} d Q_{[1,2],[5,0]}\right. \\
& \left.\left.+d Q_{[5,6],(1,2]} d Q_{[1,2],(3,5]} d Q_{[1,2,8],(5,6,7]}+d Q_{[5,6],(1,2]} d Q_{[1,2],(3,6])} d Q_{[1,2,5],(3,7,8]}\right) q_{2}\right) \\
& -d Q_{[1,2],\{4,5]}\left(-d Q_{[3,5,0],(1,2,4]} d Q_{[1,2],(5,0)}+d Q_{[1,2,3],(4,5,6]} d Q_{[3,0],(1,2]}\right)^{2} \\
& \left(\mathrm{dQ}_{[1,2,8],(5,0,8]} \mathrm{d} \mathrm{Q}_{[8,0],[1,2]}-\mathrm{d} \mathrm{Q}_{[5,0,8],[1,2,8]} \mathrm{d} Q_{[1,2],[5,0]}\right) q_{1} q_{2}{ }^{2} A_{3,4}^{2} q_{4} \\
& -\left(d Q_{[1,2,8],[5,6,8]} d Q_{[5,0],(2,2]}-d Q_{[5,0,8],[1,2,8]} d Q_{[1,2],(5,0]}\right) \\
& \left(d Q_{[3,0],\{1,2]}^{2} d Q_{[1,2],(3,5]} d Q_{[1,2,3],[4,5,6]} d Q_{[1,2,3],(3,5,0]}+d Q_{[5,6],[1,2]}^{2} d Q_{[1,2,3],(3,5,6]}^{2} d Q_{[1,2],(4,5]}\right. \\
& -d Q_{[5,6],(1,2]} d Q_{[1,2],(3,5]} d Q_{[1,2,3],(3,5,0]} d Q_{[3,5,6],(1,2,1)} d Q_{[1,2],(5,6]} \\
& -2 d Q_{[5,6],[1,2]} d Q_{[1,2],[3,5]} d Q_{[1,2,3],[4,5,6]} d Q_{[1,2],(3,6]} d Q_{[3,5,6],[1,2,3]} \\
& -d Q_{[5,6],(1,2]} d Q_{[1,2],[4,5]} d Q_{[1,2],(5,6]} d Q_{[3,5,6],(1,2,3]} d Q_{[1,2,3],(3,5,6]} \\
& \left.+2 \mathrm{dQ}_{[1,2],(3,5]} \mathrm{dQ} Q_{[1,2],(5,8)}^{2} \mathrm{dQ}_{[3,5,6),(1,2,4]} \mathrm{dQ}_{[3,5,6],(1,2,3]}\right) q_{2} A_{3,4}^{2} q_{4} \\
& +d Q_{[5,6],[1,2]}^{2} d Q_{[1,2],(5,6]}^{2}\left(-d Q_{[5,6,8],[1,2,7]} d Q_{[1,2],[4,5]} d Q_{[1,2],[5,6]}\right. \\
& \left.+d Q_{[5,6],(1,2]} d Q_{[1,2],(4,5]} d Q_{[1,2,8],(5,6,7]}+d Q_{[1,2,5],(4,7,8]} d Q_{[5,6],[1,2]} d Q_{[1,2],(5,6]}\right) q_{1} \\
& \left.d Q_{[5,6],[1,2]}^{2} d Q_{[1,2],[3,5]} d Q_{[1,2],[5,6]}^{2}\left(d Q_{[1,2,8],(5,6,8]} d Q_{[5,6],[1,2]}-d Q_{[5,6,8],[1,2,8]} d Q_{[1,2],(5,6]}\right) q_{4}\right)
\end{aligned}
$$

Notice that 3.89 is a function of $q_{1}, q_{2}, q_{4}, A_{3,4}^{2}$ and the data. We say that 3.89 is a general equation because its coefficients are functions of a general data matrix, $Q$. Later, we shall work with data from a phantom. A phantom is the solution to the forward problem for a given set of transition probabilities. Data from a phantom are real numbers, whereas general data are variables, $Q_{i, j}$. We can solve 3.89 for $A_{3,4}^{2}$ in terms of $q_{1}, q_{2}, q_{4}$ and the data. Similarly, we can solve the three other former cubic equations for $A_{3,3}^{2}, A_{7,7}^{2}$, and 
$A_{7,8}^{2}$. Each of these solutions is a function of three of the $q_{i} s$ and the data. Substituting these solutions into the four (previously) quadratic equations we get four highly nonlinear equations in $q_{1}, q_{2}, q_{3}, q_{4}$. Because the coefficients in these equations are so cumbersome only caricatures are shown below

$$
\begin{aligned}
& q_{3}\left(a_{2}+q_{3} a_{3}\right)\left(q_{4} a_{4}+q_{3}\right)\left(q_{2} a_{3}+a_{6}\right)\left(q_{2} a_{10}+a_{20}+q_{1} a_{21}\right)\left(q_{1}+q_{2} a_{4}\right) a_{1} \\
& \left(q_{3}{ }^{2} q_{2} a_{7}+q_{2} q_{3}{ }^{2} q_{4} a_{8}+q_{3} q_{2} a_{9}+q_{3} q_{3} q_{4} a_{10}+q_{2} a_{11}+q_{2} q_{4} a_{12}+\right. \\
& \left.q_{3} a_{13}+q_{3}^{2} a_{14}+a_{15}+q_{3} q_{4} a_{16}+q_{4} a_{17}+q_{3}^{2} q_{4} a_{18}\right) \\
& q_{3}\left(a_{2}+q_{3} a_{3}\right)\left(q_{3} q_{4} a_{24}+q_{3} a_{25}+q_{4} a_{26}\right)\left(q_{4} a_{4}+q_{3}\right)\left(q_{2} a_{5}+a_{6}\right)\left(q_{1}+q_{2} a_{4}\right) a_{23} \\
& \left(q_{2}{ }^{2} q_{1} a_{27}+q_{1} q_{2} q_{3} a_{28}+q_{1} q_{2} a_{29}+q_{1} q_{3} a_{30}+q_{1} a_{31}+q_{2}{ }^{2} q_{1} q_{3} a_{32}+\right. \\
& \left.q_{2}^{2} q_{3} a_{33}+a_{34}+q_{3} q_{2} a_{35}+q_{2}{ }^{2} a_{36}+q_{2} a_{37}+q_{3} a_{38}\right) \\
& q_{1}\left(q_{4} a_{40}+a_{41}\right)\left(q_{4} a_{12}+a_{43}+q_{3} a_{44}\right)\left(q_{4} a_{4}+q_{3}\right)\left(a_{45}+q_{1} a_{46}\right)\left(q_{1}+q_{2} a_{4}\right) a_{39} \\
& \left(q_{4} q_{1}{ }^{2} q_{2} a_{47}+q_{1}{ }^{2} q_{4} a_{48}+q_{1}{ }^{2} q_{2} a_{48}+q_{1}{ }^{2} a_{50}+q_{1} q_{4} a_{51}+q_{4} q_{1} q_{2} a_{52}+\right. \\
& \left.q_{1} q_{2} a_{53}+q_{1} a_{54}+q_{2} q_{4} a_{55}+a_{56}+q_{2} a_{57}+q_{4} a_{58}\right) \\
& q_{1} a_{59}\left(q_{4} a_{40}+a_{41}\right)\left(q_{4} a_{4}+q_{3}\right)\left(a_{45}+q_{1} a_{46}\right)\left(q_{1} q_{2} a_{46}+q_{1} a_{72}+q_{2} a_{73}\right) \\
& \left(q_{1}+q_{2} a_{4}\right)\left(q_{1} a_{60}+q_{1} q_{4} a_{61}+q_{1} q_{3} a_{62}+q_{1} q_{4}^{2} a_{63}+q_{1} q_{3} q_{4} a_{64}+\right. \\
& \left.q_{1} q_{3} q_{4}{ }^{2} a_{65}+a_{66}+q_{4} a_{67}+q_{3} a_{68}+q_{3} q_{4} a_{69}+q_{3} q_{4}{ }^{2} a_{70}+q_{4}{ }^{2} a_{71}\right)
\end{aligned}
$$

where each of the $a_{i} \mathrm{~S}$ is a polynomial in minors of the general data matrix, $Q$, and the "relevant" term is the last one. The zero valued, or relevant, terms of each of these four equations is a twelve term polynomial, involves only three of the $q_{i} s$, and is linear in one of the $q_{i} s$. The roles of the four $q_{i} s$ occur cyclically. Solving for two of the variables (linearly) and replacing the result into the remaining two equations we get two nonlinear equations of 35 terms each. All of this can be done with the general equations!! Solving equations 3.90 and 3.91 for $q_{1}$ and $q_{2}$ and subsituting into 3.92 and 3.93 yields 


$$
\begin{aligned}
& b_{28}+q_{3} b_{3}+q_{3}^{2} b_{1}+q_{3}^{3} b_{19}+q_{3}^{4} b_{20}+q_{3}^{5} b_{34}+q_{3} b_{35}+ \\
& q_{2} b_{2}+q_{2} q_{3} b_{3}+q_{2} q_{3}{ }^{2} b_{4}+q_{2} q_{3}{ }^{3} b_{21}+q_{2} q_{3}{ }^{4} b_{22}+q_{2} q_{3}{ }^{5} b_{28}+q_{2} q_{3}{ }^{8} b_{6}+ \\
& q_{2}{ }^{2} b_{13}+q_{2}{ }^{2} q_{3} b_{14}+q_{2}{ }^{2} q_{3}{ }^{2} b_{12}+q_{2}{ }^{2} q_{3}{ }^{3} b_{23}+q_{2}{ }^{2} q_{3}{ }^{4} b_{24}+q_{2}{ }^{2} q_{3}{ }^{5} b_{27}+q_{2}{ }^{2} q_{3}{ }^{8} b_{9}+ \\
& q_{2}{ }^{3} b_{11}+q_{2}{ }^{3} q_{3} b_{17}+q_{2}{ }^{3} q_{3}{ }^{2} b_{18}+q_{2}{ }^{3} q_{3}{ }^{3} b_{32}+q_{2}{ }^{3} q_{3}{ }^{4} b_{28}+q_{2}{ }^{3} q_{3}{ }^{8} b_{29}+q_{2}{ }^{3} q_{3}{ }^{6} b_{7}+ \\
& q_{2}{ }^{4} b_{10}+q_{2}{ }^{4} q_{3} b_{16}+q_{2}{ }^{4} q_{3}{ }^{2} b_{18}+q_{2}{ }^{4} q_{3}{ }^{3} b_{33}+q_{2}{ }^{4} q_{3}{ }^{4} b_{30}+q_{2}{ }^{4} q_{3}{ }^{5} b_{31}+q_{2}{ }^{4} q_{3}{ }^{6} b_{8}
\end{aligned}
$$

and

$$
\begin{aligned}
& b_{65}+q_{2} b_{37}+q_{2}{ }^{2} b_{44}+q_{2}{ }^{3} b_{42}+q_{2}{ }^{4} b_{41}+q_{2}{ }^{3} b_{88}+q_{2}{ }^{8} b_{60}+ \\
& q_{3} b_{38}+q_{2} q_{3} b_{40}+q_{2}{ }^{2} q_{3} b_{46}+q_{2}{ }^{3} q_{3} b_{49}+q_{2}{ }^{4} q_{3} b_{48}+q_{2}{ }^{5} q_{3} b_{68}+q_{2}{ }^{6} q_{3} b_{70}+ \\
& q_{3}{ }^{2} b_{30}+q_{3}{ }^{2} q_{2} b_{30}+q_{2}{ }^{2} q_{3}{ }^{2} b_{43}+q_{3}{ }^{2} q_{2}{ }^{3} b_{30}+q_{3}{ }^{2} q_{2}{ }^{4} b_{17}+q_{3}{ }^{2} q_{2}{ }^{5} b_{37}+q_{3}{ }^{2} q_{2}{ }^{6} b_{68}+ \\
& q_{3}{ }^{3} b_{51}+q_{3}{ }^{3} q_{2} b_{53}+q_{3}{ }^{3} q_{2}{ }^{2} b_{55}+q_{3}{ }^{3} q_{2}{ }^{3} b_{63}+q_{3}{ }^{3} q_{2}{ }^{5} b_{59}+q_{3}{ }^{3} q_{2}{ }^{4} b_{64}+q_{3}{ }^{3} q_{2}{ }^{6} b_{67}+ \\
& q_{3}{ }^{4} b_{32}+q_{3}{ }^{4} q_{2} b_{54}+q_{3}{ }^{4} q_{2}{ }^{2} b_{30}+q_{3}{ }^{4} q_{2}{ }^{3} b_{61}+q_{3}{ }^{4} q_{2}{ }^{4} b_{62}+q_{3}{ }^{4} q_{2}{ }^{5} b_{48}+q_{3}{ }^{4} q_{2}{ }^{6} b_{60}
\end{aligned}
$$

where the $b_{i} s$ are polynomials in minors of the data matrix. Thus far, our data have been the symbols $Q_{i, j}$, where $i, j=1, \ldots, 8$. The size of the polynomials (or rather the coefficients) in the above equations prohibits further computation with a general data set. During preliminary work on this problem, the author implemented this algorithm on several phantoms. The author used a very general phantom: MAPLE's random number generator was used to assign numerical values to transition probabilities. These values did not necessarily satisfy conditions 3.13 and 3.14 .

Taking the resultant of 3.94 and 3.95 yields 3.96 , a huge polynomial equation in one variable. In our numerical tests we have always observed that 3.96 factors to have the same form, regardless of the variable with which we take the resultant. The following caricature $\mathrm{cf}$ an equation shows the form of the resultants. 


$$
\begin{aligned}
& c_{1}\left(q_{3}{ }^{2} c_{40}+q_{3} c_{41}+c_{42}\right)^{2}\left(q_{3}{ }^{2} c_{43}+q_{3} c_{44}+c_{48}\right)^{2}\left(q_{3}{ }^{2} c_{40}+q_{3} c_{47}+c_{48}\right)^{2} \\
& \left(q_{3}^{2} c_{49}+q_{3} c_{30}+c_{51}\right)^{2}\left(q_{3} c_{2}+c_{3}\right) \\
& \left(q_{3}{ }^{3} c_{8}+c_{39}+q_{3}{ }^{4} c_{7}+q_{3}{ }^{5} c_{8}+q_{8}{ }^{17} c_{17}+q_{3}{ }^{30} c_{18}+q_{3}{ }^{8} c_{19}+\right. \\
& q_{3}{ }^{22} c_{20}+q_{3}{ }^{32} c_{21}+q_{3}{ }^{31} c_{22}+q_{3}{ }^{18} c_{23}+q_{3}{ }^{11} c_{24}+q_{3}{ }^{10} c_{23}+q_{3}{ }^{23} c_{20}+ \\
& q_{3}{ }^{19} c_{27}+q_{3}{ }^{34} c_{28}+q_{3}{ }^{35} c_{29}+q_{3}{ }^{20} c_{30}+q_{3}{ }^{0} c_{31}+q_{3}{ }^{12} c_{32}+q_{3}{ }^{13} c_{33}+ \\
& q_{3}{ }^{14} c_{34}+q_{3}{ }^{15} c_{35}+q_{3}{ }^{16} c_{36}+q_{3}{ }^{7} c_{37}+q_{3}{ }^{21} c_{38}+q_{3}{ }^{23} c_{12}+q_{3}{ }^{28} c_{13}+ \\
& \left.q_{3}{ }^{27} c_{14}+q_{3}{ }^{28} c_{15}+q_{3}{ }^{29} c_{10}+q_{3} c_{4}+q_{3}{ }^{2} c_{5}+q_{3}{ }^{0} c_{9}+q_{3}{ }^{20} c_{10}+q_{3}{ }^{24} c_{11}\right)
\end{aligned}
$$

where the $c_{i} s$ are complicated functions of the data and the relevant term is the only linear term in $q_{3}$.

The author completed this computation on several phantoms and in each case the relevant factor was linear $\left(q_{3} c_{2}+c_{3}\right)$ and gave the solution $q_{3}=-c_{3} / c_{2}$. Once $q_{3}$ is computed, it is possible to compute $q_{2}$ by substituting the solution for $q_{3}$ into 3.94 or 3.95 Next we can substitute the values of $q_{2}$ and $q_{3}$ into the solutions for $q_{1}$ and $q_{4}$ obtained from 3.90 and 3.91. Once the values for the $g_{1} 8$ are found, $A_{3,4}^{2}, A_{3,3}^{2}, A_{7,7}^{2}$, and $A_{7,8}^{2}$ can be computed from 3.89 and its counterparts. Therefore, we have $A_{3,4}, A_{3,3}, A_{7,7}$, and $A_{7,8}$ modulo signs. We can use this to solve 3.88 for $A_{4,4}, A_{4,3}, A_{8,7}$, and $A_{8,8}$ up to signs.

In order to assign the proper signs to $A_{3,3}, A_{3,4}, A_{4,3}$, and $A_{4,4}$ recall that since $A=P_{h o}^{-1}$ and $A$ is a block matrix,

$$
\frac{1}{A_{3,3} A_{4,4}-A_{3,4} A_{4,3}}\left[\begin{array}{cc}
A_{4,4} & -A_{3,4} \\
-A_{4,3} & A_{3,3}
\end{array}\right]=\left[\begin{array}{ll}
e 21 w & e 21 s \\
n 21 w & n 21 s
\end{array}\right]
$$

For a solution to be physically viable all of the transition probabilities must be positive. For example, we know that

$$
\operatorname{sgn}\left(A_{3,3} A_{4,4}-A_{3,4} A_{4,3}\right)=\operatorname{sgn}\left(A_{3,3}\right)=\operatorname{sgn}\left(A_{4,4}\right)=-\operatorname{sgn}\left(A_{4,3}\right)=-\operatorname{sgn}\left(A_{3,4}\right)
$$

We can use 3.98 to compute the signs of $A_{3,3}, A_{4,4}, A_{3,4}$, and $A_{4,3}$. 


\subsubsection{Writing the equations for general $n$}

For $n>2$ the problem becomes worse even though the governing matrix equation, 2.8, looks the same. Although there are always $4 n$ incoming and outgoing states for a larger $n \times n$ system, there are many more hidden states. Including incoming states, there are four states per pixel (plus $4 n$ outgoing states). Since there are $n^{2}$ pixels there are $4 n^{2}$ incoming and hidden states. That leaves $4 n^{2}-4 n$ hidden states. See figure 3.14. Only when $n=2$ is the number of hidden states equal to the number of incoming and outgoing states. For a $n \times n$ system $P_{i o}$ is a $4 n \times 4 n$ matrix and $P_{h h}$ is a $\left(4 n^{2}-4 n\right) \times\left(4 n^{2}-4 n\right)$ matrix. $P_{i h}$ is a $4 n \times\left(4 n^{2}-4 n\right)$ matrix and $P_{h o}$ is a $\left(4 n^{2}-4 n\right) \times 4 n$ matrix. For $n \geq 4$ the governing equations are so horribly large and nonlinear that MAPLE cannot even solve the forward problem analytically. (Inverting $\left(I-P_{h h}\right)$ is too much for MAPLE.) In order to begin work on the inverse problem one must somehow cut this monstrosity down to size.

Even if MAPLE were able to handle the equations for any large $n \times n$ system the algorithm described in section 3.2.1 is doomed to failure. $P_{h o}$ is not invertible since it is not even square. One would like to preserve the "squareness" of the transition submatrices as well as reduce the complexity of the problem. A recursive approach allowing only one layer of hidden states at any recursion level achieves both goals. The recursive algorithm described below decomposes the system into subsystems which are subsequently decomposed into subsystems of their own. A system is broken into subsystems by ignoring most of its hidden states. No matter how one decomposes the system, the new system must adhere to the consistency conditions discussed in section 3.1.

For any square system, notice that if we choose one horizontal and one vertical barrier there are exactly $4 n$ hidden states associated with these barriers. (Each of the barriers is associated with two rank deficient submatrices of rank $n$. The vertical barrier is associated with a right-left as well as a left-right submatrix; the horizontal barrier is associated with a top-bottom as well as a bottom-top submatrix.) Recall that there are exactly $4 n$ incoming and $4 n$ outgoing states. 
ronsider the example in figure 3.15. The $4 \times 4$ array of pixels has been divided into four subarrays, labeled 11, 12, 21, and 22. There are 16 incoming states and 16 outgoing states. There are 16 relevant hidden states, those associated with the barriers. The incoming states which send photons into a subarray are considered to be adjacent only to hidden and outgoing states which send photons out of that subarray. Similarly, hidden states which send photons from one subarray into a second subarray are adjacent only to hidden states which send photons from the second subarray into any other subarray. Finally, hidden states which send photons into a subarray are adjacent only to those outgoing states which send photons out of that subarray. As in the base case, it is assumed that photons can only travel directly from one state to adjacent states.

The governing matrix equation may be rewritten as the following:

$$
\left(Q-P_{i 0}\right) A\left(I-P_{h h}\right)-P_{i h}=\theta
$$

where $Q$ is the data matrix and $P_{i o}, P_{i h}, P_{h o}$, and $P_{h h}$ are probability transition matrices for this modified system and $A=P_{h o}^{-1}$. Although 3.99 looks the same as in the $2 \times 2$ example, the transition matrices are very different. They have nonzero entries wherever it is possible to travel from one state to another without leaving the subarray in which the first state puts the photon. The shortest possible path between states in this modified system may require that the photon travel several steps in the original system. The most important thing to notice is that these modified transition probabilities are the data for the subarrays. Once again, the transition matrices $P_{h o}$ and $P_{i o}$ share block diagonal structures and $P_{h h}$ and $P_{i h}$ share off diagonal block structures. In fact, replacing nonzero entries in the transition matrices for the $2 \times 2$ system with dense $n / 2 \times n / 2$ blocks and zeros in the $2 \times 2$ system with sparse $n / 2 \times n / 2$ blocks gives the structure of the modified transition matrices. Also, $A$ has the same structure as $P_{h o}$ and the same changes of variables which were used to solve the $2 \times 2$ problem may be used here. 


$$
\begin{aligned}
W & =A P_{h h} \\
X & =P_{i 0} A \\
Y & =P_{i 0} W-P_{i h}
\end{aligned}
$$

Finally, notice that $X$ has the same zero structure as $P_{h o}$ and $P_{i o}$ and that $Y$ and $W$ have identical zero structures as $P_{i h}$ and $P_{h h}$. The governing equation may be rewritten as

$$
Q(A-W)-(X-Y)=\Theta
$$

Just as in the $2 \times 2$ problem, there are redundant equations in the governing matrix equation and the columns of 3.101 are decoupled homogeneous systems of linear equations. As before, one may solve for the $W_{i, j} s, X_{i, j} s$, and $Y_{i, j} s$ in terms of the $A_{i, j} s$. From these solutions, one can write down the transition probabilities for the modified $4 \times 4$ system in terms of the $A_{i, j} s$. This exhausts the supply of equations given by the governing matrix equation for the modified system.

Let the data matrices for the subarrays be denoted as $Q 11, Q 12, Q 21, Q 22$. The entries of the transition matrices $P_{i o}, P_{i h}, P_{h h}$, and $P_{h o}$ may be written as functions of the entries of $A$ and are the data in $Q 11, Q 12, Q 21$, and $Q 22$. Once we recover these data matrices we can tackle each subarray separately. There are consistency conditions amongst the data for each of the subarrays. These conditions provide some highly nonlinear equations which can be used to solve for some of the $A_{i, j}$ 's in terms of the remaining $A_{i, j}$ 's. We cannot hope to recover all $16 * 4^{2}$ parameters. Some extra conditions must be found, somewhere. Once the data for each of the four subsystems is found the procedure is repeated on each of the four subsystems. This recursion continues until the $2 \times 2$ "base case" is reached. The algorithm described in section 3.2.1 is then used to glean as much useful information from the base case as is possible. Notice that there has been no mention of any identifications in this section. This method of solving the equations is absolutely general. 


\subsection{3 $4 \times 4$ problem}

The recursive algorithm described above is developed in detail for a $4 \times 4$ system.

\subsubsection{Solving for $P_{i h}, P_{h h}, P_{i o}$, and $P_{h o}$ in terms of $A$}

The algorithm for solving the $4 \times 4$ problem described below requires only one level of recursion and gives a completely general solution. In other words, the author makes no assumptions about the physical properties of the system; no identifications of the form 3.54 are made. There are $16 * 16=256$ unknown transition probabilities and (as we saw in section 3.1) only 160 independent data. In sections 3.2.3.1 and 3.2.3.2 we find a 96 parameter family of solutions to the $4 \times 4$ problem.

We start by labeling the states for the $4 \times 4$ system as in figure 3.15 , and all of the $2 \times 2$ subsystems as in figure 2.1. The transition matrices for the modified system are sparse block matrices. These matrices are larger than their $2 \times 2$ counterparts, but have similar block structures. The nonzero subblocks of the transition matrices are shown below, starting with $P_{i h}$, which has an off diagonal block structure.

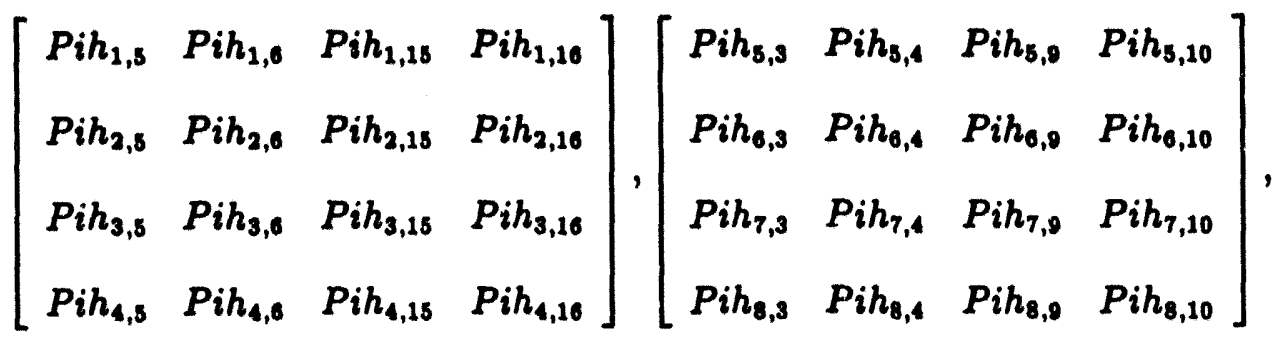

$$
\begin{aligned}
& {\left[\begin{array}{llll}
P_{i h_{9,7}} & P_{i h_{9,8}} & P_{i h_{9,13}} & P_{i h_{0,14}} \\
P_{i h_{10,7}} & P_{i h_{10,8}} & P_{i h_{10,13}} & P_{i h_{10,14}} \\
P_{i h_{11,7}} & P_{i h_{11,8}} & P_{i h_{11,13}} & P_{i h_{11,14}} \\
P_{i h_{12,7}} & P_{i h_{12,8}} & P_{i h_{12,13}} & P_{i h_{12,14}}
\end{array}\right] \text { and }} \\
& {\left[\begin{array}{llll}
P_{i h_{13,1}} & P i h_{13,2} & P i h_{13,11} & P i h_{13,12} \\
P_{i h_{14,1}} & P i h_{14,2} & P i h_{14,11} & P i h_{14,12} \\
P_{i h_{15,1}} & P i h_{15,2} & P i h_{15,11} & P_{i h_{15,12}} \\
P_{i h_{16,1}} & P i h_{16,2} & P i h_{16,11} & P i h_{16,12}
\end{array}\right]}
\end{aligned}
$$


$P_{h h}$ has the same block structure as $P_{i h}$,

$\left[\begin{array}{llll}P h h_{9,7} & P h h_{0,8} & P h h_{0,13} & P h h_{0,14} \\ P h h_{10,7} & P h h_{10,8} & P h h_{10,13} & P h h_{10,14} \\ P h h_{11,7} & P h h_{11,8} & P h h_{11,13} & P h h_{11,14} \\ P h h_{12,7} & P h h_{12,8} & P h h_{12,13} & P h h_{12,14}\end{array}\right],\left[\begin{array}{llll}P h h_{19,1} & P h h_{13,2} & P h h_{18,11} & P h h_{13,12} \\ P h h_{14,1} & P h h_{14,2} & P h h_{14,11} & P h h_{14,12} \\ P h h_{18,1} & P h h_{18,2} & P h h_{18,11} & P h h_{18,12} \\ P h h_{16,1} & P h h_{10,2} & P h h_{16,11} & P h h_{10,12}\end{array}\right]$

$\left[\begin{array}{llll}P h h_{1,5} & P h h_{1,0} & P h h_{1,18} & P h h_{1,16} \\ P h h_{2,5} & P h h_{2,6} & P h h_{3,15} & P h h_{2,16} \\ P h h_{3,5} & P h h_{3,6} & P h h_{3,18} & P h h_{3,16} \\ P h h_{4,5} & P h h_{4,0} & P h h_{4,18} & P h h_{4,16}\end{array}\right]$ and $\left[\begin{array}{llll}P h h_{8,3} & P h h_{8,4} & P h h_{5,9} & P h h_{8,10} \\ P h h_{0,3} & P h h_{0,4} & P h h_{0,9} & P h h_{0,10} \\ P h h_{7,3} & P h h_{7,4} & P h h_{7,0} & P h h_{7,10} \\ P h h_{0,3} & P h h_{8,4} & P h h_{8,0} & P h h_{8,10}\end{array}\right]$,

$P_{i o}$, however, is block diagonal,

\begin{tabular}{|c|c|c|c|c|c|c|c|c|}
\hline$P_{i o_{1,1}}$ & $P_{i O_{1,2}}$ & $P_{i O_{1,3}}$ & $P_{i O_{1,4}}$ & & $P_{i O_{5,5}}$ & Pios, & Pio $_{6,7}$ & Pion, \\
\hline $\mathrm{PiO}_{2,1}$ & $\mathrm{PiO}_{2,2}$ & $\mathrm{PiO}_{2,3}$ & $\mathrm{PiO}_{2,4}$ & & $\mathrm{PiO}_{6, \mathrm{~B}}$ & $\mathrm{PiO}_{6,0}$ & $P_{i 0_{6,7}}$ & $P_{i 0_{6,8}}$ \\
\hline$P_{i O_{3,1}}$ & $\mathrm{PiO}_{3,2}$ & $\mathrm{PiO}_{3,3}$ & $P_{i O_{3,4}}$ & & $P_{i O_{\eta, 5}}$ & $\mathrm{PiO}_{7,6}$ & $\mathrm{PiO}_{7,7}$ & $P_{i O_{7,8}}$ \\
\hline $\mathrm{PiO}_{4,1}$ & $\mathrm{PiO}_{4,2}$ & $P_{i O_{4,3}}$ & $P_{i O_{4,4}}$ & & $\mathrm{Pio}_{8,5}$ & $P_{i O_{8,0}}$ & $P_{i O_{8,7}}$ & PiO $_{8,8}$ \\
\hline
\end{tabular}

$\left[\begin{array}{llll}\text { Pio }_{9,9} & \text { Pio }_{9,10} & \text { Pio }_{9,11} & \text { Pio }_{9,12} \\ \text { Pio }_{10,9} & \text { Pio }_{10,10} & \text { Pio }_{10,11} & \text { Pio }_{10,12} \\ \text { Pio }_{11,9} & \text { Pio }_{11,10} & \text { Pio }_{11,11} & \text { Pio }_{11,12} \\ \text { Pio }_{12,9} & \text { Pio }_{12,10} & P_{i o_{12,11}} & P_{i o_{12,12}}\end{array}\right]$ and

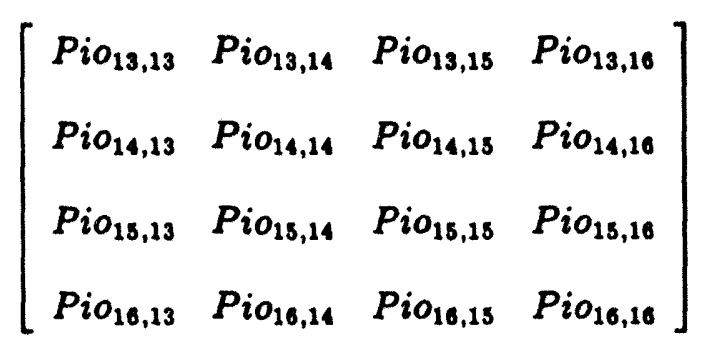


as is $P_{h o}$

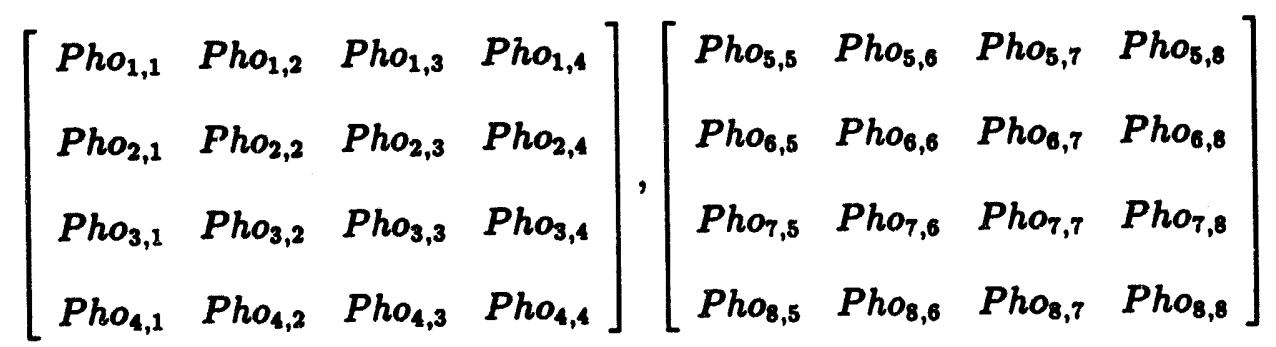

$$
\begin{aligned}
& {\left[\begin{array}{cccc}
\text { Pho }_{9,9} & \text { Pho }_{9,10} & \text { Pho }_{9,11} & \text { Pho } \\
\text { Pho }_{10,9} & P h o_{10,10} & \text { Pho }_{10,11} & P h o_{10,12} \\
\text { Pho }_{11,9} & P h o_{11,10} & \text { Pho }_{11,11} & P h o_{11,12} \\
\text { Pho }_{12,9} & P h o_{12,10} & \text { Pho }_{12,11} & P h o_{12,12}
\end{array}\right]}
\end{aligned}
$$

and

$$
\left[\begin{array}{llll}
P_{h o_{13,13}} & P h o_{13,14} & P h o_{13,15} & P h o_{13,16} \\
P o_{14,13} & P h o_{14,14} & P h o_{14,15} & P h o_{14,16} \\
\text { Pho }_{15,13} & P h o_{15,14} & P h o_{15,15} & P h o_{15,16} \\
\text { Pho }_{16,13} & P h o_{16,14} & P h o_{16,15} & P h o_{16,16}
\end{array}\right]
$$

See figure 3.16 for a few examples of paths taken into account by the modified transition probabilities displayed above.

Just as for the $2 \times 2$ problem, we may rewrite the governing equations for the $4 \times 4$ problem. Assuming that the matrix $P_{h o}$ is invertible, make the change of variables $A=P_{h o}^{-1}$ where the nonzero subblocks of $A$ are 


$$
\begin{gathered}
{\left[\begin{array}{llll}
A_{1,1} & A_{1,2} & A_{1,3} & A_{1,4} \\
A_{2,1} & A_{2,2} & A_{2,3} & A_{2,4} \\
A_{3,1} & A_{3,2} & A_{3,3} & A_{3,4} \\
A_{4,1} & A_{4,2} & A_{4,3} & A_{4,4}
\end{array}\right],\left[\begin{array}{llll}
A_{5,5} & A_{5,6} & A_{5,7} & A_{5,8} \\
A_{6,5} & A_{6,6} & A_{6,7} & A_{6,8} \\
A_{7,5} & A_{7,6} & A_{7,7} & A_{7,8} \\
A_{8,5} & A_{8,6} & A_{8,7} & A_{8,8}
\end{array}\right]} \\
{\left[\begin{array}{lllll}
A_{9,9} & A_{9,10} & A_{9,11} & A_{9,12} \\
A_{10,9} & A_{10,10} & A_{10,11} & A_{10,12} \\
A_{11,9} & A_{11,10} & A_{11,11} & A_{11,12} \\
A_{12,9} & A_{12,10} & A_{12,11} & A_{12,12}
\end{array}\right],\left[\begin{array}{llll}
A_{13,13} & A_{13,14} & A_{13,15} & A_{13,16} \\
A_{14,13} & A_{14,14} & A_{14,15} & A_{14,16} \\
A_{15,13} & A_{15,14} & A_{15,15} & A_{15,16} \\
A_{16,13} & A_{16,14} & A_{16,15} & A_{16,16}
\end{array}\right]}
\end{gathered}
$$

This allows us to write

$$
\left(Q-P_{i o}\right) A\left(I-P_{h h}\right)-P_{i h}=\Theta
$$

Once again we may make the following changes of variables:

$$
\begin{aligned}
W & =A P_{h h} \\
X & =P_{i o} A \\
Y & =P_{i o} W-P_{i h}
\end{aligned}
$$

The resulting matrices $X, W$, and $Y$ have very special block structures - the same block structures as the transition matrices above. $X$ has the same zero structure as $P_{h o}$ and $P_{i o} . W$ and $Y$, however, have the same zero structure as $P_{i h}$ and $P_{h h}$ : Once the changes of variables in equation 3.104 have been made, the governing equations become the familiar

$$
Q(A-W)-(X-Y)=\Theta
$$

Just as in the $2 \times 2$ case the columns of 3.105 come in groups. Only in this case four of the columns correspond to the same matrix equation. The eleventh through fourteenth columns of 3.105 are written below. 


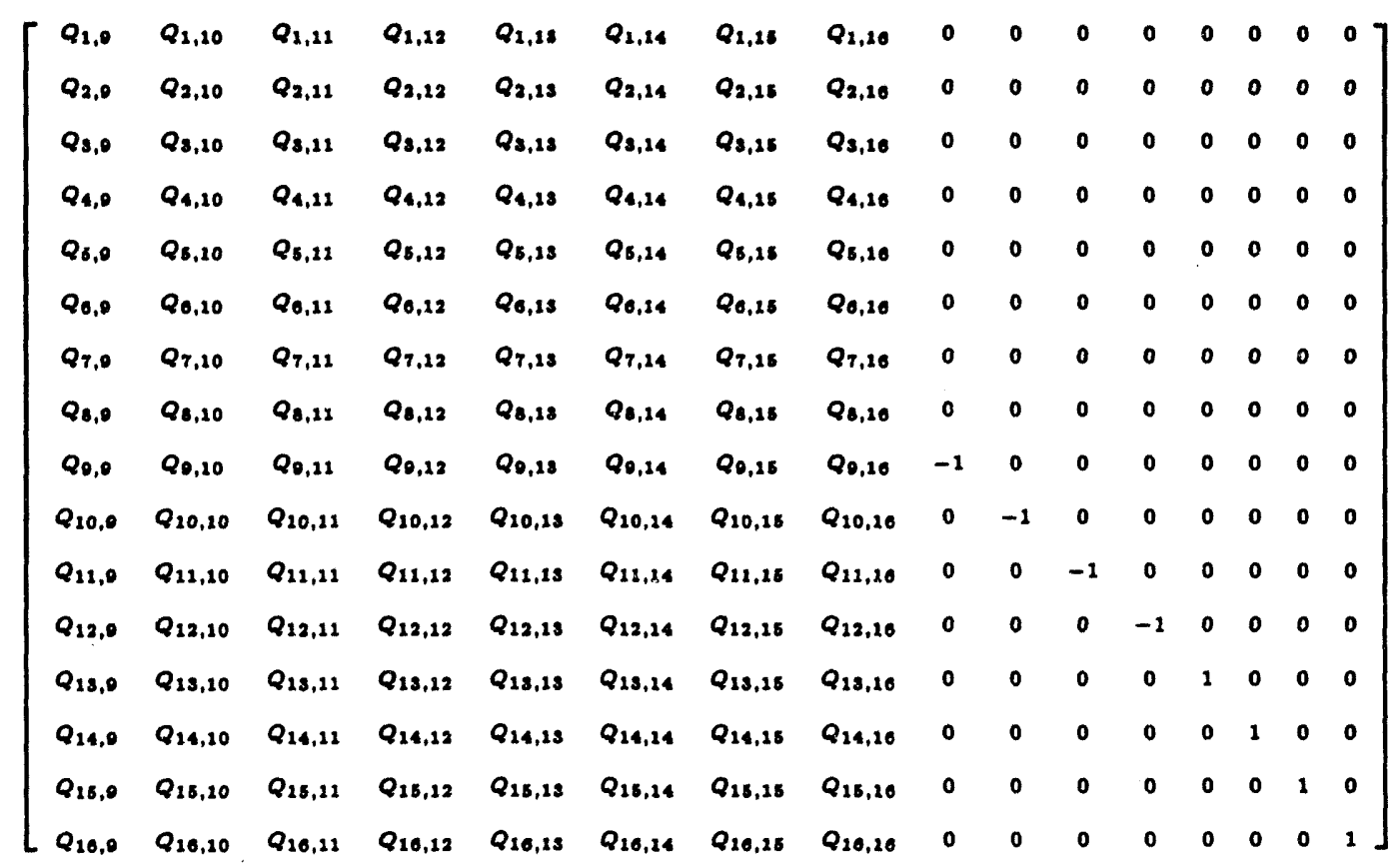

$\left[\begin{array}{cccc}A_{0,12} & A_{0,12} & -W_{0,13} & -W_{0,14} \\ A_{10,11} & A_{10,12} & -W_{16,13} & -W_{10,14} \\ A_{11,11} & A_{11,12} & -W_{11,13} & -W_{11,14} \\ A_{12,11} & A_{12,12} & -W_{12,13} & -W_{12,14} \\ -W_{13,12} & -W_{18,12} & A_{13,13} & A_{13,14} \\ -W_{14,11} & -W_{14,12} & A_{14,13} & A_{14,14} \\ -W_{13,11} & -W_{16,12} & A_{16,13} & A_{13,14} \\ -W_{16,11} & -W_{16,12} & A_{16,13} & A_{10,14} \\ X_{0,11} & X_{0,12} & Y_{0,13} & Y_{0,14} \\ X_{10,11} & X_{10,12} & Y_{10,13} & Y_{10,14} \\ X_{11,11} & X_{11,12} & Y_{11,13} & Y_{11,14} \\ X_{12,11} & X_{12,12} & Y_{12,13} & Y_{12,14} \\ Y_{13,11} & Y_{13,12} & X_{13,13} & X_{13,14} \\ Y_{14,11} & Y_{14,12} & X_{14,13} & X_{14,14} \\ Y_{16,11} & Y_{16,12} & X_{16,13} & X_{16,14} \\ Y_{16,11} & Y_{16,12} & X_{10,13} & X_{10,14}\end{array}\right]=\Theta$

Just as in the $2 \times 2$ case, not all of these equations are independent. Consistency conditions force the $8 \times 8$ upper left submatrix of the first matrix in equation 3.106 to be of rank four or less. We can solve for the $W_{i, j}$ 's, $X_{i, j}$ 's, and $Y_{i, j}$ 's in terms of the $A_{i, j}$ 's. Solving the first two columns of 3.106 is equivalent to solving the equation 


\begin{tabular}{|c|c|c|c|c|c|c|c|c|c|c|c|c|c|}
\hline$Q_{B, 13}$ & $Q_{8,24}$ & $Q_{6,15}$ & Q8,10 & 0 & 0 & 0 & 0 & 0 & 0 & 0 & 07 & $-w_{13,11}$ & $-W_{13,12}$ \\
\hline$Q 0,13$ & $Q 0,14$ & $Q_{0,18}$ & $Q_{0,10}$ & 0 & 0 & 0 & 0 & 0 & 0 & 0 & 0 & $-W_{11,13}$ & $-W_{14,12}$ \\
\hline$Q_{7,13}$ & $Q_{7,24}$ & $Q_{7,2 B}$ & $Q 7,10$ & 0 & 0 & $0^{\circ}$ & 0 & 0 & 0 & 0 & 0 & $-W_{16,11}$ & $-W_{16,12}$ \\
\hline$Q_{0,23}$ & $Q_{8,14}$ & $Q 8,16$ & $Q 8,10$ & 0 & 0 & 0 & 0 & 0 & 0 & 0 & 0 & $-W_{16,11}$ & $-W_{10,12}$ \\
\hline$Q_{0,13}$ & $Q_{0,14}$ & $Q 0,28$ & $Q 0,10$ & -1 & 0 & 0 & 0 & 0 & 0 & 0 & 0 & $x_{0,11}$ & $x_{0,12}$ \\
\hline$Q_{10,13}$ & $Q_{10,14}$ & $Q_{10,26}$ & $Q_{10,10}$ & 0 & -1 & 0 & 0 & 0 & 0 & 0 & 0 & $x_{10,11}$ & $x_{10,12}$ \\
\hline$Q_{21,13}$ & $Q_{11,14}$ & $Q_{11,18}$ & $Q_{11,10}$ & 0 & 0 & -1 & 0 & 0 & 0 & 0 & 0 & $x_{11,11}$ & $x_{11,12}$ \\
\hline$Q_{12,13}$ & $Q_{12,14}$ & $Q_{12,16}$ & $Q_{12,16}$ & 0 & 0 & 0 & -1 & 0 & 0 & 0 & 0 & $x_{12,11}$ & $x_{12,12}$ \\
\hline$Q_{13,13}$ & $Q_{13,24}$ & $Q_{13,18}$ & $Q_{18,10}$ & 0 & 0 & 0 & 0 & 1 & 0 & 0 & 0 & $\gamma_{13,11}$ & $Y_{13,12}$ \\
\hline$Q_{14,13}$ & $Q_{14,14}$ & $Q_{24,13}$ & $Q_{14,10}$ & 0 & 0 & 0 & 0 & 0 & 1 & 0 & 0 & $Y_{14,12}$ & $Y_{14,12}$ \\
\hline$Q_{15,13}$ & $Q_{18,14}$ & $Q_{18,16}$ & $Q_{16,10}$ & 0 & 0 & 0 & 0 & 0 & 0 & 1 & 0 & $Y_{16,11}$ & $Y_{18,12}$ \\
\hline$Q_{10,13}$ & $Q_{16,14}$ & $Q_{10,15}$ & $Q_{10,10}$ & 0 & 0 & 0 & 0 & 0 & 0 & 0 & 1 & $Y_{10,11}$ & $Y_{16,12}$ \\
\hline
\end{tabular}

$$
=\left[\begin{array}{llll}
Q_{5,9} & Q_{5,10} & Q_{5,11} & Q_{5,12} \\
Q_{6,9} & Q_{6,10} & Q_{6,11} & Q_{6,12} \\
Q_{7,9} & Q_{7,10} & Q_{7,11} & Q_{7,12} \\
Q_{8,9} & Q_{8,10} & Q_{8,11} & Q_{8,12} \\
Q_{9,9} & Q_{9,10} & Q_{9,11} & Q_{9,12} \\
Q_{10,9} & Q_{10,10} & Q_{10,11} & Q_{10,12} \\
Q_{11,9} & Q_{11,10} & Q_{11,11} & Q_{11,12} \\
Q_{12,9} & Q_{12,10} & Q_{12,11} & Q_{12,12} \\
Q_{13,9} & Q_{13,10} & Q_{13,11} & Q_{13,12} \\
Q_{14,9} & Q_{14,10} & Q_{14,11} & Q_{14,12} \\
Q_{15,9} & Q_{15,10} & Q_{15,11} & Q_{15,12} \\
Q_{16,9} & Q_{16,10} & Q_{16,11} & Q_{16,12}
\end{array}\right]\left[\begin{array}{ll}
A_{9,11} & A_{9,12} \\
A_{10,11} & A_{10,12} \\
A_{11,11} & A_{11,12} \\
A_{12,11} & A_{12,12}
\end{array}\right]
$$

Solving for $W, X$, and $Y$ in terms of $A$ exhausts the supply of equations given by the governing matrix equation. Since $A$ is invertible, one may now solve for the entries in $P_{i h}, P_{h h}, P_{i o}$, and $P_{h o}$ in terms of the data and $A_{i, j}$ 's. The forms of the solutions are similar among variables from the same transition matrix; samples of solutions in terms of $A_{i, j}$ 's for one variable from each matrix are listed below. We start with the simplest solutions, those in $P_{h o}$ : 
68

$$
P h o_{3,4}=-\frac{d A_{[1,2,4]},[1,2,3]}{d A_{[1,2,3,4],[1,2,3,4]}}
$$

The next simplest solutions are those for entries of $P_{h h}$.

$$
\begin{aligned}
& P h h_{3,15}= \\
& \text { - }\left(d A _ { [ 1 , 2 , 3 ] , [ 1 , 2 , 4 ] } \left(\mathrm{dQ}_{[5,6,7,8],[1,2,3,13]} A_{13,15}+\mathrm{dQ}_{[5,6,7,8],[1,2,3,14]} A_{14,15}\right.\right. \\
& \left.+\mathrm{dQ}_{[5,6,7,8],[1,2,3,15]} A_{15,15}+\mathrm{dQ}_{[5,6,7,8],[1,2,3,16]} A_{16,15}\right) \\
& +d A_{[1,2,4],[1,2,4]}\left(\mathrm{dQ}_{[5,6,7,8],[1,2,4,13]} A_{13,15}+\mathrm{dQ}_{[5,6,7,8],[1,2,4,14]} A_{14,15}\right. \\
& \left.+\mathrm{dQ}_{[5,6,7,8],[1,2,4,15]} A_{15,15}+\mathrm{dQ}_{[5,6,7,8),(1,2,4,16]} A_{16,15}\right) \\
& +d A_{[1,3,4],[1,2,4]}\left(\mathrm{dQ}_{[5,6,7,8],[1,3,4,13]} A_{13,15}+\mathrm{dQ}_{[5,6,7,8],[1,3,4,14]} A_{14,15}\right. \\
& \left.+\mathrm{dQ}_{[5,6,7,8],[1,3,4,15]} A_{15,15}+\mathrm{dQ}_{[5,6,7,8],(1,3,4,16]} A_{16,15}\right) \\
& +d A_{[2,3,4],(1,2,4]}\left(\mathrm{dQ}_{[5,6,7,8],[2,3,4,13]} A_{13,15}+\mathrm{dQ}_{[5,6,7,8],[2,3,4,14]} A_{14,15}\right. \\
& \left.\left.+\mathrm{d} Q_{[5,6,7,8],(2,3,4,15]} A_{15,15}+\mathrm{dQ}_{[5,6,7,8],\{2,3,4,16]} A_{16,15}\right)\right) / \\
& \mathrm{dQ}_{[5,6,7,8],[1,2,3,4]} d A_{[1,2,3,4],[1,2,3,4]}
\end{aligned}
$$

The solutions for the entries of $P_{i o}$ are a little bit longer:

$$
\begin{aligned}
& P i o_{5,6}=-\frac{1}{d A_{[5,6,7,8],[5,6,7,8]}} \\
& \left(d A _ { [ 5 , 7 , 8 ] , [ 6 , 7 , 8 ] } \left(\mathrm{dQ}_{[5,13,14,15,16],[1,2,3,4,5]} A_{5,5}+\right.\right. \\
& \mathrm{dQ}_{[5,13,14,15,16],[1,2,3,4,6]} A_{6,5}+\mathrm{dQ} Q_{[5,13,14,15,16],[1,2,3,4,7]} A_{7,5}+ \\
& \left.\mathrm{dQ}_{[5,13,14,15,16],[1,2,3,4,8]} A_{8,5}\right) / \mathrm{dQ}_{[13,14,15,16],[1,2,3,4]}- \\
& d A_{[5,7,8],[5,7,8]}\left(\mathrm{dQ}_{[5,13,14,15,16],[1,2,3,4,5]} A_{5,6}+\right. \\
& \mathrm{dQ}_{[5,13,14,15,16],(1,2,3,4,6]} A_{6,6}+\mathrm{dQ}_{[5,13,14,15,16],[1,2,3,4,7]} A_{7,6}+ \\
& \left.\mathrm{dQ}_{[5,13,14,15,16],[1,2,3,4,8]} A_{8,6}\right) / \mathrm{dQ}_{[13,14,15,18],[1,2,3,4]}- \\
& d A_{[5,7,8],(5,6,7]}\left(\mathrm{dQ}_{[5,13,14,15,16],[5,9,10,11,12]} A_{5,8}+\right. \\
& \mathrm{dQ}_{[5,13,14,15,16],[6,9,10,11,12]} A_{6,8}+\mathrm{dQ}_{[5,13,14,15,16],[7,9,10,11,12]} A_{7,8}+ \\
& \left.\mathrm{dQ}_{[5,13,14,15,16],[8,9,10,11,12]} A_{8,8}\right) / \mathrm{dQ}_{[13,14,15,16],[9,10,11,12]} \\
& d A_{[5,7,8],[5,6,8]}\left(\mathrm{dQ}_{[5,13,14,15,16],[5,9,10,11,12]} A_{5,7}+\right. \\
& \mathrm{dQ}_{[5,13,14,15,16],[6,9,10,11,12]} A_{6,7}+\mathrm{dQ}_{[5,13,14,15,16],\{7,9,10,11,12]} A_{7,7}+ \\
& \left.\left.\mathrm{d} \mathrm{Q}_{[5,13,14,15,16],[8,9,10,11,12]} A_{8,7}\right) / \mathrm{dQ}_{[13,14,15,16],[9,10,11,12]}\right)
\end{aligned}
$$


69

Solutions for the entries of $P_{\text {in }}$ are of the form:

$$
\begin{aligned}
& \operatorname{Pih}_{10,14}=\frac{1}{\mathrm{dQ}_{[3,6,7,8],(9,10,11,12]}} \\
& \left(\mathrm{dQ}_{[5,6,7,8,10],(9,10,11,12,13)} A_{13,14}+\mathrm{dQ}_{[3,6,7,8,10],(9,10,11,12,14]} A_{14,14}+\right. \\
& \left.\mathrm{dQ}_{[5,6,7,8,10],\{0,10,11,12,15]} A_{15,14}+\mathrm{dQ}_{[5,6,7,8,10],(9,10,11,12,10]} A_{16,14}\right)+ \\
& \frac{1}{\mathrm{~d} Q_{[8,6,7,8],\{9,10,11,12]} d A_{[0,10,11,12],[8,10,11,12]}}\left(\left(\mathrm{dQ}_{[5,6,7,8],[8,11,12,13]} A_{13,14}+\right.\right. \\
& \mathrm{dQ}_{[5,6,7,8],(9,11,12,14]} A_{14,14}+\mathrm{dQ}_{[5,0,7,8],[9,11,12,15]} A_{15,14}+ \\
& \left.\mathrm{dQ}_{[8,6,7,8],[0,11,12,16]} A_{16,14}\right) \\
& \left(d A _ { [ 0 , 1 1 , 1 2 ] , ( 8 , 1 0 , 1 1 ] } \left(\mathrm{dQ}_{[5,6,7,8,10],(9,13,14,15,16]} A_{9,12}\right.\right. \\
& +\mathrm{dQ}_{[5,6,7,8,10],[10,13,14,16,16]} A_{10,12}+d Q_{[5,6,7,8,10],[11,13,14,15,16]} A_{11,12} \\
& \left.+\mathrm{dQ}_{[5,6,7,8,10],(12,13,14,15,16]} A_{12,12}\right) / \mathrm{dQ}_{[5,6,7,8],[13,14,15,16]} \\
& -d A_{[0,11,12],(9,10,12]}\left(\mathrm{d}_{[5,6,7,8,10],\{9,13,14,15,16]} A_{9,11}\right. \\
& +\mathrm{dQ}_{[5,6,7,8,10],[10,13,14,15,16]} A_{10,11}+\mathrm{d} \mathrm{Q}_{[5,6,7,8,10],[11,13,14,15,16]} A_{11,11} \\
& \left.+\mathrm{dQ}_{[5,6,7,8,10],[12,13,14,16,16]} A_{12,11}\right) / \mathrm{dQ}_{[8,6,7,8],[13,14,16,16]} \\
& +d A_{[9,11,12],[9,11,12]}\left(\mathrm{dQ}_{[10,13,14,15,16],[5,6,7,8,9]} A_{9,10}\right. \\
& +\mathrm{dQ}_{[10,13,14,15,16],[5,6,7,8,10]} A_{10,10}+\mathrm{dQ}_{[10,13,14,15,16],[5,6,7,8,11]} A_{11,10} \\
& \left.+\mathrm{dQ}_{[10,13,14,15,16],(5,6,7,8,12]} A_{12,10}\right) / \mathrm{dQ}_{[13,14,15,16],\{5,8,7,8]} \\
& -d A_{[9,11,12],[10,11,12]}\left(\mathrm{dQ}_{[10,13,14,15,16],[5,6,7,8,10]} A_{10,9}\right. \\
& +\mathrm{dQ}_{[10,13,14,15,16],[5,6,7,8,9]} A_{9,9}+\mathrm{dQ} \mathrm{Q}_{[10,13,14,15,16],(5,6,7,8,11]} A_{11,9} \\
& \left.\left.+\mathrm{d} \mathrm{Q}_{[10,13,14,15,16],(5,6,7,8,12]} A_{12,8}\right) / \mathrm{dQ}_{[13,14,15,10],[5,8,7,8]}\right)
\end{aligned}
$$


70

$$
\begin{aligned}
& +\left(\mathrm{dQ}_{[5,6,7,8],[0,10,12,13]} A_{13,14}+\mathrm{d} \mathrm{Q}_{[3,6,7,8],[8,10,12,14]} A_{14,14}\right.
\end{aligned}
$$

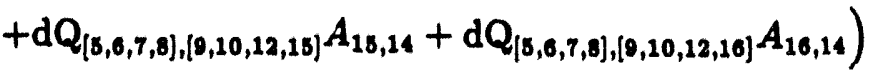

$$
\begin{aligned}
& \left(d A _ { [ 0 , 1 0 , 1 2 ] , \{ 0 , 1 0 , 1 1 ] } \left(\mathrm{dQ}_{[5,0,7,8,10],[9,13,14,15,10]} A_{0,12}\right.\right. \\
& +\mathrm{d} Q_{[8,0,7,8,10],[10,13,14,15,16]} A_{10,12}+\mathrm{dQ}_{[5,6,7,8,10],(11,13,14,16,16]} A_{11,12} \\
& \left.+\mathrm{d} \mathrm{Q}_{[5,6,7,8,10],[12,13,14,18,16]} A_{12,12}\right) / \mathrm{dQ}_{[8,6,7,8],[13,14,15,16]} \\
& -d A_{[0,10,12],[0,10,12]}\left(\mathrm{dQ}_{[5,6,7,8,10],[9,13,14,15,16]} A_{9,11}\right. \\
& +\mathrm{d} \mathrm{Q}_{[5,6,7,8,10],[10,13,14,15,16]} A_{10,11}+\mathrm{dQ}_{[5,6,7,8,10],(11,13,14,16,16]} A_{11,11} \\
& \left.+\mathrm{dQ}_{[3,6,7,8,10],(12,13,14,15,16]} A_{12,11}\right) / \mathrm{dQ}_{[5,6,7,8],[13,14,15,16]} \\
& +d A_{[9,10,12],[9,11,12]}\left(\mathrm{dQ}_{[10,13,14,15,16],[5,6,7,8,9]} A_{9,10}\right. \\
& +\mathrm{d} \mathrm{Q}_{[10,13,14,15,16],\{5,6,7,8,10]} A_{10,10}+\mathrm{dQ}_{[10,13,14,15,16],\{5,6,7,8,11]} A_{11,10} \\
& \left.+\mathrm{dQ}_{[10,13,14,15,16],(5,6,7,8,12]} A_{12,10}\right) / \mathrm{dQ}_{[13,14,15,16],\{5,6,7,8]} \\
& -d A_{[0,10,12],[10,11,12]}\left(\mathrm{dQ}_{[10,13,14,15,16],[5,6,7,8,10]} A_{10,9}\right. \\
& +\mathrm{dQ}_{[10,13,14,15,16],[5,6,7,8,9]} A_{9,9}+\mathrm{d} \mathrm{Q}_{[10,13,14,15,16],[5,6,7,8,11]} A_{11,9} \\
& \left.\left.+\mathrm{d} \mathrm{Q}_{[10,13,14,15,16],[5,6,7,8,12]} A_{12,9}\right) / \mathrm{dQ}_{[13,14,15,16],(5,6,7,8]}\right) \\
& +\left(\mathrm{d} \mathrm{Q}_{[5,6,7,8],[10,11,12,13]} A_{13,14}+\mathrm{d} \mathrm{Q}_{[5,6,7,8],[10,11,12,14]} A_{14,14}\right. \\
& \left.+\mathrm{dQ}_{[5,6,7,8],[10,11,12,15]} A_{15,14}+\mathrm{dQ}_{[5,6,7,8],[10,11,12,16]} A_{16,14}\right)
\end{aligned}
$$


71

$$
\begin{aligned}
& \left(d A _ { [ 1 0 , 1 1 , 1 2 ] , [ 0 , 1 0 , 1 1 ] } \left(\mathrm{d} Q_{[0,6,7,8,10],[0,13,14,15,16]} A_{9,12}\right.\right. \\
& +\mathrm{d} Q_{[5,0,7,8,10],(10,13,14,18,10]} A_{10,12}+\mathrm{dQ}_{[8,6,7,8,10],\{11,13,14,18,10]} A_{11,12} \\
& \left.+\mathrm{dQ}_{[5,6,7,8,10],(12,13,14,16,16]} A_{12,12}\right) / \mathrm{dQ}_{[5,6,7,8],(13,14,15,16]} \\
& -d A_{[10,11,12],[9,10,12]}\left(\mathrm{dQ}_{[5,6,7,8,10],(9,13,14,16,16]} A_{9,11}\right. \\
& +d Q_{[8,0,7,8,10],(10,13,14,15,16]} A_{10,11}+d Q_{[5,6,7,8,10],[11,13,14,15,16]} A_{11,11} \\
& \left.+\mathrm{d} \mathrm{Q}_{[5,6,7,8,10],(12,13,14,16,16]} A_{12,12}\right) / \mathrm{dQ}_{[5,6,7,8],(13,14,15,16]} \\
& +d A_{[10,11,12],[9,11,12]}\left(\mathrm{dQ}_{[10,13,14,16,18],[5,6,7,8,9]} A_{9,10}\right. \\
& +\mathrm{dQ}_{[10,13,14,15,16],(5,6,7,8,10]} A_{10,10}+\mathrm{dQ}_{[10,13,14,15,16],(5,6,7,8,11]} A_{11,10} \\
& \left.+\mathrm{dQ}_{[10,13,14,15,16],(5,6,7,8,12]} A_{12,10}\right) / \mathrm{dQ}_{[13,14,15,16],(5,6,7,8]} \\
& -d A_{[10,11,12],[10,11,12]}\left(\mathrm{d} \mathrm{Q}_{[10,13,14,15,16],[6,6,7,8,10]} A_{10,9}\right. \\
& +\mathrm{dQ}_{[10,13,14,15,16],[5,6,7,8,9]} A_{9,9}+\mathrm{d} \mathrm{Q}_{[10,13,14,15,16],(5,6,7,8,11]} A_{11,9} \\
& \left.\left.+\mathrm{dQ}_{[10,13,14,15,16],(5,6,7,8,12]} A_{12,9}\right) / \mathrm{dQ}_{[13,14,15,16],[5,6,7,8]}\right) \\
& +\left(\mathrm{dQ}_{[5,6,7,8],[8,10,11,13]} A_{13,14}+\mathrm{dQ}_{[5,0,7,8],[0,10,11,14]} A_{14,14}\right. \\
& \left.+\mathrm{dQ}_{[5,6,7,8],(9,10,11,15]} A_{15,14}+\mathrm{dQ}_{[5,6,7,8],(9,10,11,16]} A_{16,14}\right) \\
& \left(d A _ { [ 9 , 1 0 , 1 1 ] , [ 9 , 1 0 , 1 1 ] } \left(\mathrm{d} \mathrm{Q}_{[5,6,7,8,10],[9,13,14,15,16]} A_{9,12}\right.\right. \\
& +\mathrm{dQ}_{[5,6,7,8,10],[10,13,14,15,16]} A_{10,12}+\mathrm{dQ}_{[5,6,7,8,10],[11,13,14,15,10]} A_{11,12} \\
& \left.+\mathrm{dQ}_{[5,6,7,8,10],(12,13,14,15,16]} A_{12,12}\right) / \mathrm{dQ}_{[5,6,7,8],(13,14,15,16]} \\
& -d A_{[9,10,11],[9,10,12]}\left(\mathrm{dQ}_{[5,6,7,8,10],[9,13,14,15,16]} A_{9,11}\right. \\
& +\mathrm{dQ}_{[5,6,7,8,10],[10,13,14,15,16]} A_{10,11}+\mathrm{d} \mathrm{Q}_{[5,6,7,8,10],[11,13,14,15,16]} A_{11,11} \\
& \left.+\mathrm{dQ}_{[5,6,7,8,10],(12,13,14,15,16]} A_{12,11}\right) / \mathrm{dQ}_{[5,6,7,8],[13,14,15,16]} \\
& +d A_{[9,10,11],[9,11,12]}\left(\mathrm{dQ}_{[10,13,14,15,16],[5,6,7,8,9]} A_{9,10}\right. \\
& +\mathrm{dQ}_{[10,13,14,15,16],[5,6,7,8,10]} A_{10,10}+\mathrm{dQ}_{[10,13,14,15,16],[5,6,7,8,11]} A_{11,10} \\
& \left.+\mathrm{dQ}_{[10,13,14,15,16],(5,6,7,8,12]} A_{12,10}\right) / \mathrm{dQ}_{[13,14,15,16],[5,6,7,8]} \\
& -d A_{[9,10,11],[10,11,12]}\left(\mathrm{dQ}_{[10,13,14,15,16],(5,6,7,8,10]} A_{10,9}\right. \\
& +\mathrm{dQ}_{[10,13,14,15,16],[5,6,7,8,9]} A_{9,9}+\mathrm{dQ}_{[10,13,14,15,16],[5,6,7,8,11]} A_{11,9} \\
& \left.\left.\left.+\mathrm{dQ}_{[10,13,14,15,16],[5,6,7,8,12]} A_{12,9}\right) / \mathrm{dQ}_{[13,14,15,16],[5,6,7,8]}\right)\right)
\end{aligned}
$$




\subsubsection{Eliminating $A_{i, j}$ 's}

Each of the four subsystems has an $8 \times 8$ data matrix. The data matrix for the 1,1 subsystem is shown below:

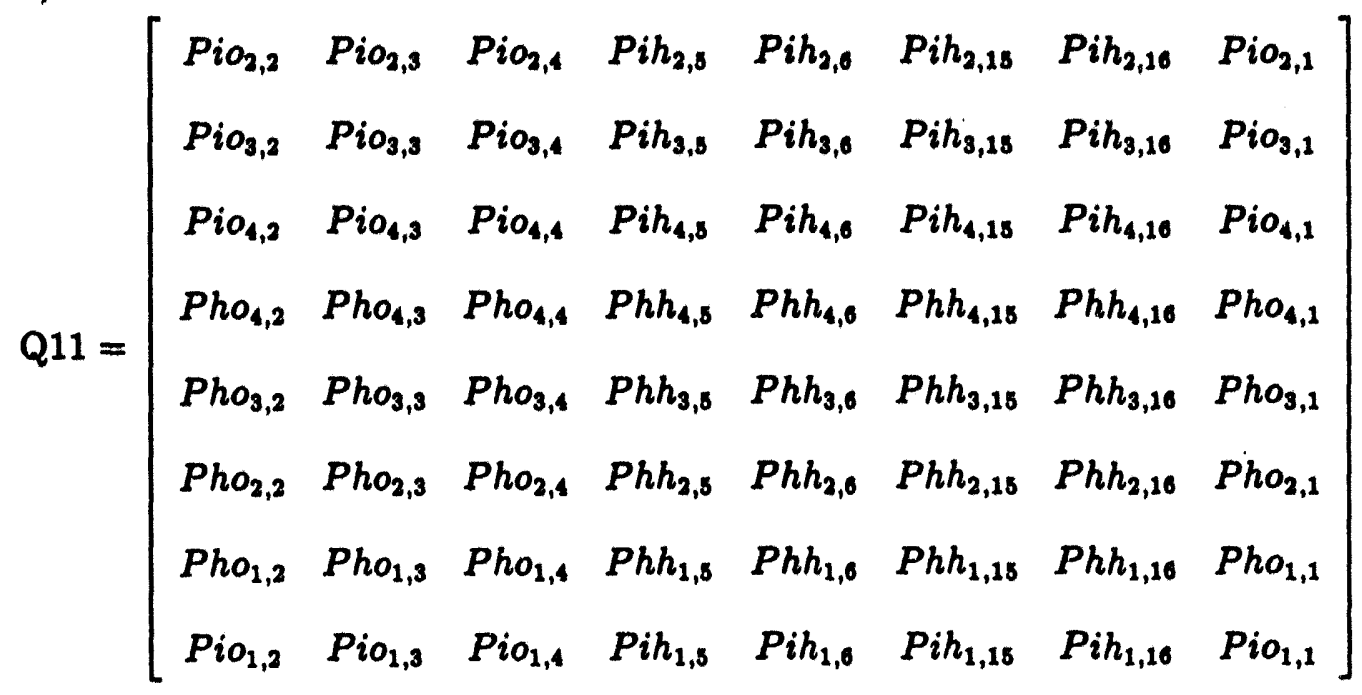

Q11 has four rank deficient submatrices. They are $4 \times 4$ submatrices of rank two (or less). Two constraints are required to force a generic vector in $\mathbb{R}^{4}$ to lie in a given two dimensional subspace. Four conditions are required, therefore, to force a generic $4 \times 4$ matrix to be of rank two. These consistency conditions upon $Q 11$ may be expressed as the vanishing of $3 \times 3$ minors. Substituting the solutions for the modified transition probabilities into these minors forces highly nonlinear polynomials of the $A_{i, j} s$ to be identically zero. These conditions will be studied in order of increasing complexity. (Clearly, the conditions which involve variables from $P_{\text {in }}$ are bound to be horrendous, so they are not considered until much later.) Eight of the conditions are identities of the form $A_{i, j}=0$. The rest reduce (at a generic point) to four term linear equations. In the rest of this section, the right-left, left-right, top-bottom, and bottom-top rank deficient submatrices are labeled as $Q i j_{r l}, Q i j_{l r}, Q i j_{t b}$, and $Q i j_{b t}$ where $i, j=1,2$. For example, 


$$
\begin{aligned}
& Q 11_{r l}=\left[\begin{array}{llll}
\mathrm{Q} 11_{8,1} & \mathrm{Q} 11_{6,2} & \mathrm{Q} 11_{8,3} & \mathrm{Q} 11_{8,4} \\
\mathrm{Q} 11_{6,1} & \mathrm{Q} 11_{6,2} & \mathrm{Q} 11_{6,3} & \mathrm{Q} 11_{6,4} \\
\mathrm{Q} 11_{7,1} & \mathrm{Q} 11_{7,2} & \mathrm{Q} 11_{7,3} & \mathrm{Q} 11_{7,4} \\
\mathrm{Q} 11_{8,1} & \mathrm{Q} 11_{8,2} & \mathrm{Q} 11_{8,3} & \mathrm{Q} 11_{8,4}
\end{array}\right]
\end{aligned}
$$

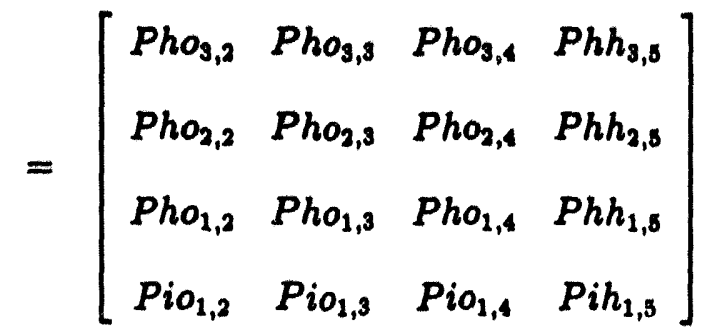

3.2.3.2.1 Identities Since $Q 11_{r l}$ is rank two, the determinant of any $3 \times 3$ minor is identically zero. Hence,

$$
\begin{aligned}
0 & =\left|\begin{array}{ccc}
\mathrm{Q} 11_{5,1} & \mathrm{Q}_{3,2} & \mathrm{Q} 11_{5,3} \\
\mathrm{Q} 11_{6,1} & \mathrm{Q} 11_{6,2} & \mathrm{Q} 11_{0,3} \\
\mathrm{Q} 11_{7,1} & \mathrm{Q} 11_{7,2} & \mathrm{Q} 11_{7,3}
\end{array}\right| \\
& =\left|\begin{array}{lll}
P h o_{3,2} & P h o_{3,3} & P h o_{3,4} \\
P h o_{2,2} & P h O_{2,3} & P h o_{2,4} \\
P h o_{1,2} & P h o_{1,3} & P h o_{1,4}
\end{array}\right|
\end{aligned}
$$

Since the solutions for entries in $P_{h o}$ in terms of $A_{i, j}$ s are simplest, 3.114 is the simplest $3 \times 3$ minor of 3.113 when the solutions in terms of $A_{i, j} s$ are substituted in. Recall that $A$ is a $16 \times 16$ block matrix, with $4 \times 4$ blocks on the diagonal. The upper left block of $A$ is the inverse of the upper left block of $P_{h o}$, and so 


$$
\begin{aligned}
& -A_{4,1}=\left|\begin{array}{lll}
P h o_{1,2} & \text { Pho }_{1,3} & \text { Pho }_{1,4} \\
\text { Pho }_{2,2} & \text { Pho }_{2,3} & \text { Pho }_{2,4} \\
\text { Pho }_{3,2} & \text { Pho }_{3,3} & \text { Pho }_{3,4}
\end{array}\right| / \text { Pho }_{[1,2,3,4],[1,2,3,4]} \\
& =\left|\begin{array}{lll}
Q 11_{5,1} & Q 11_{5,2} & Q 11_{5,3} \\
Q 11_{6,1} & Q 11_{0,2} & Q 11_{6,3} \\
Q 11_{7,1} & Q 11_{7,2} & Q 11_{7,3}
\end{array}\right| / d P h o[1,2,3,4],(1,2,3,4]
\end{aligned}
$$

The same reasoning applies to $Q 11_{b t}$ and shows that $A_{1,4}=0$. This argument also applies to the rank-deficient submatrices $Q 21_{r l}, Q 21_{t b}, Q 12_{l r}, Q 12_{b t}, Q 22_{t b}$, and $Q 22_{l r}$ and yields the following identities:

$$
\begin{aligned}
A_{1,4}=0, & A_{4,1}=0, & A_{8,8}=0, & A_{8,8}=0 \\
A_{9,12}=0, & A_{12,8}=0, & A_{19,10}=0, & A_{16,13}=0
\end{aligned}
$$

So really the upper left subblock of $A$ looks like

$$
\left[\begin{array}{cccc}
A_{1,1} & A_{1,2} & A_{1,3} & 0 \\
A_{2,1} & A_{2,2} & A_{2,3} & A_{2,4} \\
A_{3,1} & A_{3,2} & A_{3,3} & A_{3,4} \\
0 & A_{4,2} & A_{4,3} & A_{4,4}
\end{array}\right]
$$

For larger systems there are even more zero valued $A_{i, j} \mathrm{~s}$. In the first recursive step in the algorithm for the $8 \times 8$ problem $A$ is a $32 \times 32$ block diagonal matrix with four $8 \times 8$ blocks along the diagonal. For exactly the same reason that the blocks of $A$ in the $4 \times 4$ problem have zero valued corners the blocks of $A$ for the $8 \times 8$ problem have three zero valued entries in each of their off diagonal corners. The upper left block has the zero structure : 


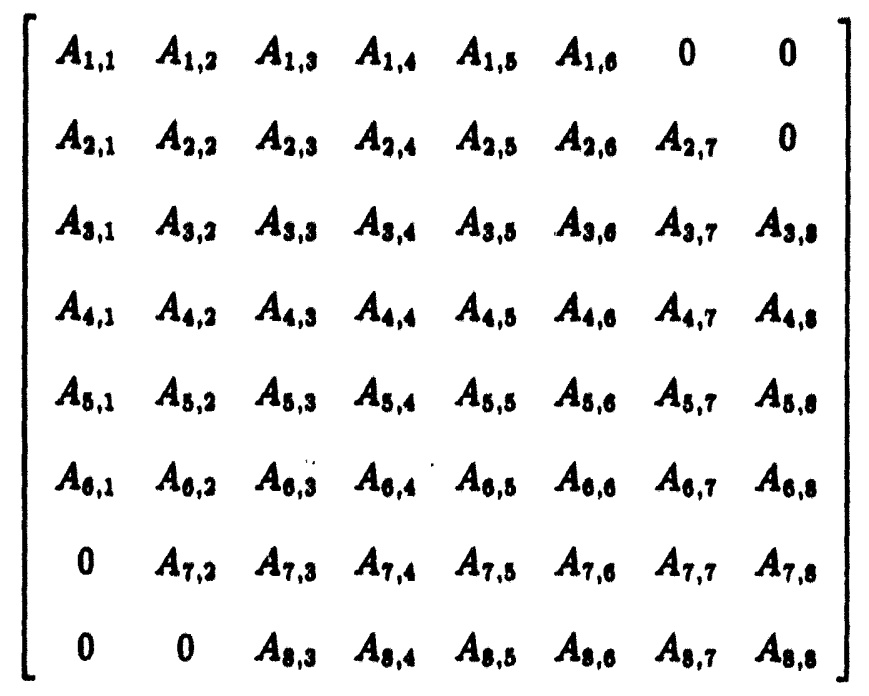

In general, for a $n \times n$ problem where $n=2^{k}$ for some whole number $k$, the matrix $A$ at the first level in this recursive algorithm has four $n \times n$ blocks and each of these blocks contains $\sum_{j=1}^{(k-1)} j=\frac{h(h-1)}{2}$ zeros in its off diagonal corners.

3.2.3.2.2 Easy Conditions Notice that there are sixteen $3 \times 3$ minors of the matrix Q11,l. Each rank deficient submatrix like $Q 11_{\text {rl }}$ yields at most four independent consistency conditions. Since we already know that $A_{4,1} \equiv 0$, we can hope to get at most three more independent conditions from setting the $3 \times 3$ minors of $Q 11_{r l}$ to zero. When the other fifteen $3 \times 3$ minors are first written down, they seem highly nonlinear, but upon closer inspection they proved to be quite simple. Grabmann relations may be used to simplify the equations. Although we need not consider all fifteen remaining minors, we do so for the $Q 11_{r l}$ submatrix. (In later sections minors of other matrices will turn out to be so cumbersome that we only consider an independent set of minors.)

"Easy" Conditions before Graßmann

Eight of the minors factor very easily. The minor $d Q 11_{r l(2,3,4),(1,2,3]}$ factors to become 
76

$$
\begin{aligned}
& d Q_{[8,0,7,8],(13,14,18,16]} \\
& \left(-d A_{[1,2,4],[1,2,3]} \quad d A_{[1,3,4],[1,3,4]} \quad d A_{[1,2,3],[2,3,4]}+\right. \\
& d A_{[1,2,4],(2,3,4]} d A_{[1,3,4],(1,3,4]} d A_{[1,2,3],(1,2,3]} \\
& d A_{[1,2,4],[1,3,4]} \quad d A_{[1,3,4],(2,3,4]} \quad d A_{1,2,2,3],(1,2,3]}+ \\
& d A_{(1,2,4),(1,3,4)} d A_{(1,2,3),(2,3,4]} \quad d A_{(1,3,4),(1,2,3]}- \\
& d A_{[1,2,4],[2,3,4]} \quad d A_{[1,2,3],(1,3,4]} \quad d A_{(1,3,4],(1,2,3]}+ \\
& \left.d A_{[1,2,4],[1,2,3]} d A_{[1,2,3],(1,3,4]} \quad d A_{[1,3,4],\{2,3,4]}\right) \\
& \left(\mathrm{dQ}_{[1,13,14,18,16],(3,8,6,7,8]} A_{3,4}+\mathrm{d} \mathrm{Q}_{[1,13,14,18,26],[4,5,6,7,8\}} A_{4,4}+\right. \\
& \left.\mathrm{dQ}_{[1,13,14,18,16],[1,5,0,7,0]} A_{1,4}+A_{2,4} \mathrm{dQ}_{[1,13,14,15,16],(2,5,0,7,8]}\right)
\end{aligned}
$$

the minor $d Q 11_{r l \mid 1,3,4),(2,2,3)}$ becomes

$$
\begin{aligned}
& -\mathrm{d} Q_{[B, 0,7,8],[13,14,18,16]} \\
& \left(d A_{[1,2,4],[1,2,4]} \quad d A_{[1,2,3],\{2,3,4]} \quad d A_{[1,3,4],[1,2,3]}+\right. \\
& d A_{[1,2,4],[2,3,4]} \quad d A_{[1,3,4],(1,2,4]} \quad d A_{[1,2,3],(1,2,3]}- \\
& d A_{[1,2,4],[1,2,4]} \quad d A_{[1,3,4],[2,3,4]} \quad d A_{[1,2,3],\{1,2,3]}- \\
& d A_{[1,2,4],\{2,3,4]} \quad d A_{[1,2,3],[1,2,4]} \quad d A_{[1,3,4],[1,2,3]}+ \\
& d A_{[1,2,4],[1,2,3]} \quad d A_{[1,2,3],[1,2,4]} \quad d A_{[1,3,4],\{2,3,4]}- \\
& \left.d A_{[1,2,4],[1,2,3]} \quad d A_{[1,3,4],[1,2,4]} \quad d A_{[1,2,3],[2,3,4]}\right) \\
& \left(\mathrm{dQ}_{[1,13,14,15,16],(3,5,0,7,8]} A_{3,4}+\mathrm{d} Q_{[1,13,14,15,16],(4,5,6,7,8]} A_{4,4}+\right. \\
& \left.\mathrm{dQ}_{[1,13,14,15,16],(1,3,6,7,8]} A_{1,4}+A_{2,4} \mathrm{dQ}_{[1,13,14,13,16],(2,5,6,7,8]}\right)
\end{aligned}
$$


and the minor $d Q 11_{r l[1,2,4),(1,2,3)}$ is

$$
\begin{aligned}
& d Q_{[3,0,7,8],(13,16,16,10]} \\
& \left(d A_{[1,2,3),[1,2,3]} \quad d A_{[1,3,4],(1,2,4]} \quad d A_{[1,2,4],(1,3,4]}\right. \\
& d A_{(1,2,3),(1,2,3)} d A_{[1,2,4],(1,2,4]} d A_{[1,3,4],(1,3,4]}- \\
& d A_{[1,2,3],(1,2,4]} d A_{[1,3,4],(1,2,3]} d A_{[1,2,4],(1,3,4]}+ \\
& d A_{[1,2,3],[1,2,4]} d A_{[1,2,4],[1,2,3]} d A_{[1,3,4],[1,3,4]}+ \\
& d A_{[1,2,3],[1,3,4]} d A_{[1,3,4],(1,2,3]} \quad d A_{[1,2,4],[1,2,4]}- \\
& \left.d A_{[1,2,3],[1,3,4]} d A_{[1,2,4],(1,2,3]} d A_{[1,3,4],(1,2,4]}\right) \\
& \left(\mathrm{d} Q_{[1,13,14,15,16],(3,5,6,7,8]} A_{3,4}+\mathrm{d} Q_{[1,13,14,15,10],[4,5,6,7,8\}} A_{4,4}+\right. \\
& \left.\mathrm{dQ}_{[1,13,14,16,16],\{1,5,6,7,8]} A_{1,4}+A_{2,4} \mathrm{dQ}_{[1,13,14,18,16],(2,5,0,7,8]}\right)
\end{aligned}
$$

\section{"Basy" Conditions after Graßmann}

We can use Graßmann relations to simplify the minors 3.116, 3.117, and 3.118 even further. For example, the cubic term in equation 3.116 may be rewritten as

$$
\begin{aligned}
& {[1,2,4],[1,3,4]} \\
& d A_{[1,2,4],[2,3,4]}\left(-d A_{[1,2,3],[1,2,3]} d A_{[1,3,4],[1,3,4]}+d A_{[1,2,3],[1,3,4]} d A_{[1,3,4],[1,2,3]}\right)+ \\
& d A_{[1,2,4],[1,2,3]}\left(d A_{[1,2,3],[1,3,4]} d A_{[1,3,4],[2,3,4]}-d A_{[1,2,3],[2,3,4]} d A_{[1,3,4],[1,3,4]}\right)
\end{aligned}
$$

Using the matrix

$$
\left[\begin{array}{llllllll}
A_{1,1} & A_{1,2} & A_{1,3} & A_{1,4} & 1 & 0 & 0 & 0 \\
A_{2,1} & A_{2,2} & A_{2,3} & A_{2,4} & 0 & 1 & 0 & 0 \\
A_{3,1} & A_{3,2} & A_{3,3} & A_{3,4} & 0 & 0 & 1 & 0 \\
A_{4,1} & A_{4,2} & A_{4,3} & A_{4,4} & 0 & 0 & 0 & 1
\end{array}\right]
$$

we may rewrite 3.119 in Graßmann notation as 


$$
\begin{array}{rllll}
\pi_{1,3,4,7} & \left(\pi_{2,3,4,8}\right. & \left.\pi_{1,2,3,6}-\pi_{2,3,4,6} \pi_{1,2,3,8}\right)+ \\
& \pi_{2,3,4,7} & \left(-\pi_{1,3,4,6}\right. & \left.\pi_{1,2,3,8}+\pi_{1,3,4,8} \pi_{1,2,3,6}\right) \\
& \pi_{1,2,3,7} & \left(-\pi_{1,3,4,8}\right. & \left.\pi_{2,3,4,6}+\pi_{1,3,4,6} \pi_{2,3,4,8}\right)
\end{array}
$$

We may next make use of the Graßmann relations

$$
\begin{aligned}
& 0=\pi_{2,3,4,8} \pi_{1,2,3,6}-\pi_{2,3,4,1} \pi_{8,2,3,6}+\pi_{2,3,4,2} \pi_{8,1,3,6}- \\
& \pi_{2,3,4,3} \pi_{8,1,2,6}+\pi_{2,3,4,6} \pi_{8,1,2,3} \\
&=\pi_{2,3,4,8} \pi_{1,2,3,6}-\pi_{2,3,4,6} \pi_{1,2,3,8}-\pi_{2,3,6,8} \pi_{1,2,3,4},
\end{aligned}
$$

$$
0=\pi_{1,3,4,8} \pi_{1,2,3,6}-\pi_{1,3,4,1} \pi_{8,2,3,6}+\pi_{1,3,4,2} \pi_{8,1,3,6}-
$$

$$
=\pi_{1,3,4,8} \pi_{1,2,3,6}-\pi_{1,3,4,6} \pi_{1,2,3,8}-\pi_{1,3,6,8} \pi_{1,2,3,4}, \text { and }
$$

$\mathrm{O}=\pi_{1,3,4,8} \pi_{2,3,4,6}-\pi_{1,3,4,2} \pi_{8,3,4,6}+\pi_{1,3,4,3} \pi_{8,2,4,6}-$

$$
\begin{gathered}
\pi_{1,3,4,4} \pi_{8,2,3,6}+\pi_{1,3,4,6} \pi_{8,2,3,4} \\
=\pi_{1,3,4,8} \pi_{2,3,4,8}-\pi_{1,3,4,6} \pi_{2,3,4,8}+\pi_{3,4,6,8} \pi_{1,2,3,4}
\end{gathered}
$$

Using these relations, the expression in 3.121 may be simplified as

$$
\begin{aligned}
& \pi_{1,3,4,7} \pi_{2,3,6,8} \pi_{1,2,3,4}- \\
& \pi_{2,3,4,7} \pi_{1,3,6,8} \pi_{1,2,3,4}+\pi_{1,2,3,7} \pi_{3,4,6,8} \pi_{1,2,3,4}
\end{aligned}
$$

Finally, we can make use of the Graßmanin relation

$$
\begin{gathered}
\pi_{1,3,4,7} \pi_{2,3,6,8}-\pi_{2,3,4,7} \pi_{1,3,6,8}+ \\
\pi_{1,2,3,7} \pi_{3,4,6,8}-\pi_{3,6,7,8} \pi_{1,2,3,4}=0
\end{gathered}
$$

to simplify 3.125 . When equation 3.126 is used to simplify equation 3.125 , the result is a product 


$$
\pi_{3,6,7,8} \quad \pi_{1,2,3,4}^{2}
$$

which equals $A_{1,3} d A_{[1,2,3,4],[1,2,3,4]}^{2}$ in the original notation. We can write the minor $d Q 11_{r l[2,3,4],[1,2,3]}$ as

$$
\begin{aligned}
& -\mathrm{dQ}_{[5,6,7,8],[13,14,15,16]} A_{1,3} \quad d A_{[1,2,3,4],[1,2,3,4]}^{2} \\
& \left(\mathrm{dQ}_{[1,13,14,15,16],(3,5,6,7,8]} A_{3,4}+\mathrm{dQ} \mathrm{Q}_{[1,13,14,15,16],[4,5,6,7,8]} A_{4,4}+\right. \\
& \left.\mathrm{dQ}_{[1,13,14,15,16],[1,5,6,7,8]} A_{1,4}+A_{2,4} \mathrm{dQ}_{[1,13,14,15,16],[2,5,6,7,8]}\right)
\end{aligned}
$$

Similarly, equations 3.117 and 3.118 are equivalent to

$$
\begin{gathered}
\mathrm{dQ}_{[5,6,7,8],[13,14,15,16]} A_{1,2} \quad d A_{[1,2,3,4],[1,2,3,4]}^{2} \\
\left(\mathrm{dQ}_{[1,13,14,15,16],[3,5,6,7,8]} A_{3,4}+\mathrm{dQ}_{[1,13,14,15,16],[4,5,6,7,8]} A_{4,4}+\right. \\
\left.\quad \mathrm{d} \mathrm{Q}_{[1,13,14,15,16],[1,5,6,7,8]} A_{1,4}+A_{2,4} \mathrm{~d} Q_{[1,13,14,15,16],[2,5,6,7,8]}\right)
\end{gathered}
$$

and

$$
\begin{aligned}
& -\mathrm{d} \mathrm{Q}_{[5,0,7,8],[13,14,15,16]} A_{1,1} d A_{[1,2,3,4],[1,2,3,4]}^{2} \\
& \left(\mathrm{dQ}_{[1,13,14,15,16],[3,5,6,7,8]} A_{3,4}+\mathrm{d} \mathrm{Q}_{[1,13,14,15,16],[4,5,6,7,8]} A_{4,4}+\right. \\
& \left.\quad \mathrm{d} \mathrm{Q}_{[1,13,14,15,16],[1,5,6,7,8]} A_{1,4}+A_{2,4} \mathrm{dQ} Q_{[1,13,14,15,16],[2,5,6,7,8]}\right)
\end{aligned}
$$

respectively.

Recall that each of these minors must be identically zero, $d A_{[1,2,3,4],[1,2,3,4]} \neq 0$ and $A_{1,1}, A_{1,2}$, and $A_{1,3}$ are generically nonzero. Finally, the $4 \times 4$ minor of $Q, d Q_{[5,6,7,8],\{13,14,15,16]}$, is also generically nonzero. Hence, in each of these three minors the relevant term is the last one: 


$$
\begin{aligned}
& \mathrm{d} Q_{[1,13,14,15,16],[3,5,6,7,8]} A_{3,4}+\mathrm{d} Q_{[1,13,14,15,16],[4,5,6,7,8]} A_{4,4} \\
& \quad+\mathrm{d} \mathrm{Q}_{[1,13,14,15,16],[1,5,6,7,8]} A_{1,4}+A_{2,4} \mathrm{dQ}_{[1,13,14,15,16],[2,5,6,7,8]}=0
\end{aligned}
$$

There are three more minors which factor easily. $d Q 11_{r l[1,2,3],[1,2,4]}$ equals

$$
\begin{aligned}
\left(-A_{5,5} \mathrm{~d} Q_{[13,14,15,16],[2,3,4,5]}-A_{6,5} \mathrm{~d} Q_{[13,14,15,16],[2,3,4,6]}\right. & \\
& \left.A_{8,5} \mathrm{dQ}_{[13,14,15,16],[2,3,4,8]}-A_{7,5} \mathrm{dQ}_{[13,14,15,16],[2,3,4,7]}\right) \\
& \left(d A_{[1,3,4],[1,3,4]} d A_{[1,2,4],[2,3,4]} d A_{[2,3,4],[1,2,4]}+\right. \\
& d A_{[1,3,4],[2,3,4]} d A_{[2,3,4],[1,3,4]} d A_{[1,2,4],[1,2,4]}- \\
& d A_{[1,3,4],[1,3,4]} d A_{[2,3,4],[2,3,4]} d A_{[1,2,4],[1,2,4]}- \\
& d A_{[1,3,4],[2,3,4]} d A_{[1,2,4],[1,3,4]} d A_{[2,3,4],[1,2,4]}+ \\
& d A_{[1,3,4],[1,2,4]} d A_{[1,2,4],\{1,3,4]} d A_{[2,3,4],[2,3,4]}- \\
& \left.d A_{[1,3,4],[1,2,4]} d A_{[2,3,4],[1,3,4]} d A_{[1,2,4],[2,3,4]}\right)
\end{aligned}
$$

and $d Q 11_{r l[1,2,3],[2,3,4]}$ equals

$$
\begin{aligned}
\left(A_{5,5} \mathrm{dQ}_{[13,14,15,16],[2,3,4,5]}+A_{6,5} \mathrm{dQ}_{[13,14,15,16],[2,3,4,6]}+\right. \\
\left.A_{8,5} \mathrm{dQ}_{[13,14,15,16],[2,3,4,8]}+A_{7,5} \mathrm{dQ}_{[13,14,15,16],[2,3,4,7]}\right) \\
\left(-d A_{[1,2,4],[1,3,4]} d A_{[1,2,3],[2,3,4]} d A_{[2,3,4],[1,2,4]}-\right. \\
\quad d A_{[1,2,4],[2,3,4]} d A_{[2,3,4],[1,3,4]} d A_{[1,2,3],[1,2,4]}+ \\
\quad d A_{[1,2,4],[1,3,4]} d A_{[2,3,4],[2,3,4]} d A_{[1,2,3],[1,2,4]}+ \\
\quad d A_{[1,2,4],[2,3,4]} d A_{[1,2,3],[1,3,4]} d A_{[2,3,4],[1,2,4]}- \\
\quad d A_{[1,2,4],[1,2,4]} d A_{[1,2,3],[1,3,4]} d A_{[2,3,4],[2,3,4]}+ \\
\left.\quad d A_{[1,2,4],[1,2,4]} d A_{[2,3,4],[1,3,4]} d A_{[1,2,3],[2,3,4]}\right)
\end{aligned}
$$


and $d Q 11_{r l[1,2,3\},[1,3,4]}$ equals

$$
\begin{aligned}
\left(-A_{5,5} \mathrm{~d}\right. & Q_{[13,14,15,16],[2,3,4,5]}-A_{6,5} \mathrm{~d} \mathrm{Q}_{[13,14,15,16],[2,3,4,6]}- \\
& \left.A_{8,5} \mathrm{~d} \mathrm{Q}_{[13,14,15,16],[2,3,4,8]}-A_{7,5} \mathrm{~d} \mathrm{Q}_{[13,14,15,16],[2,3,4,7]}\right) \\
& \left(-d A_{[1,3,4],[1,3,4]} d A_{[1,2,3],[2,3,4]} d A_{[2,3,4],[1,2,4]}-\right. \\
& d A_{[1,3,4],[2,3,4]} d A_{[2,3,4],[1,3,4]} d A_{[1,2,3],[1,2,4]}+ \\
& d A_{[1,3,4],[1,3,4]} d A_{[2,3,4],[2,3,4]} d A_{[1,2,3],[1,2,4]}+ \\
& d A_{[1,3,4],[2,3,4]} d A_{[1,2,3],[1,3,4]} d A_{[2,3,4],[1,2,4]}- \\
& d A_{[1,3,4],[1,2,4]} d A_{[1,2,3],[1,3,4]} d A_{[2,3,4],[2,3,4]}+ \\
& \left.d A_{[1,3,4],[1,2,4]} d A_{[2,3,4],[1,3,4]} d A_{[1,2,3],[2,3,4]}\right)
\end{aligned}
$$

Just as before, one may use Graßmann relations to simplify the above minors. Provided that $A_{2,4}, A_{3,4}$, and $A_{4,4}$ are nonzero they yield only one relevant term:

$$
\begin{aligned}
& \left(A_{5,5} \mathrm{dQ}_{[13,14,15,16],[2,3,4,5]}+A_{6,5} \mathrm{dQ}_{[13,14,15,16],[2,3,4,6]}+\right. \\
& \left.A_{8,5} \mathrm{dQ}_{[13,14,15,16],[2,3,4,8]}+A_{7,5} \mathrm{dQ}_{[13,14,15,16],[2,3,4,7]}\right)=0
\end{aligned}
$$

The same hold true for the other rank deficient submatrices, $Q 11_{b t}, Q 21_{r l}, Q 21_{t b}$, $Q 12_{l r}, Q 12_{b t}, Q 22_{t b}$, and $Q 22_{l r}$. Each submatrix has several $3 \times 3$ minors which factor easily but these easily factorizable minors yield only two relevant equations per submatrix. Fortunately, these equations are linear in the unknowns! Recall that each of these submatrices has one $3 \times 3$ minor which yields one of the identities in 3.116. So we expect to find only one more independent relation per rank deficient submatrix. Fortunately, the remaining minors are easily cleaned up. They are sums of many terms, some of which are eqivalent to the identities just found (like 3.135) multiplied by some other term. When these identities are subtracted from one of the remaining minors, another relation amongst the $A_{i, j}$ s appears. One example is given below: 


$$
\begin{aligned}
& \mathrm{d} Q_{[5,6,7,8],[13,14,15,16]} \mathrm{d} \mathrm{Q}_{[13,14,15,16],[5,6,7,8]} d A_{[1,4],[2,4]} \\
& \left(A_{7,5} \mathrm{~d} Q_{[1,13,14,15,16],[1,2,3,4,7]}+A_{6,5} \mathrm{~d} Q_{[1,13,14,15,16],[1,2,3,4,6]}+\right. \\
& \left.A_{5,5} \mathrm{~d} Q_{[1,13,14,15,16],[1,2,3,4,5]}+\mathrm{dQ}_{[1,13,14,15,16],[1,2,3,4,8]} A_{8,5}\right)=0
\end{aligned}
$$

In fact, all of the remaining $3 \times 3$ minors of $Q 11_{r l}$ yield the same relevant term, as we might expect. Each of the other seven rank deficient submatrices that have been studied thus far yields exactly one more independent relation amongst the $A_{i, j} s$.

If the rank deficient submatrices $Q 11_{r l}, Q 11_{b t}, Q 21_{r l}, Q 21_{t b}, Q 12_{l r}, Q 12_{b t}, Q 22_{t b}$, and $Q 22_{l r}$ were all independent of each other then they would correspond to $8 * 4=32$ independent $3 \times 3$ minors. Unfortunately, this is not the case. The 24 nontrivial minors may be grouped in eight sets of three according to their unknowns. Graßmann relations may be used to show that all three equations per group are equivalent. One of the sets is shown below:

$$
\begin{aligned}
& \mathrm{dQ}_{[1,13,14,15,16],[1,2,3,4,7]} A_{7,5}+\mathrm{d} \mathrm{Q}_{[1,13,14,15,16\},[1,2,3,4,6]} A_{6,5}+ \\
& \mathrm{dQ}_{[1,13,14,15,16],[1,2,3,4,5]} A_{5,5}+\mathrm{dQ}_{[1,13,14,15,16],[1,2,3,4,8]} A_{8,5}=0 \\
& \mathrm{dQ}_{[13,14,15,16],[2,3,4,5]} A_{5,5}+\mathrm{dQ}_{[13,14,15,16],[2,3,4,6]} A_{6,5}+ \\
& \mathrm{dQ}_{[13,14,15,16],(2,3,4,8]} A_{8,5}+\mathrm{dQ}_{[13,14,15,16],[2,3,4,7]} A_{7,5}=0 \\
& \mathrm{dQ}_{[8,13,14,15,16],[1,2,3,4,7]} A_{7,5}+\mathrm{d} \mathrm{Q}_{[8,13,14,15,16],\{1,2,3,4,8]} A_{8,5}+ \\
& \mathrm{dQ}_{[8,13,14,15,16],[1,2,3,4,6]} A_{6,5}+\mathrm{dQ}_{[8,13,14,15,16],[1,2,3,4,5]} A_{5,5}=0
\end{aligned}
$$

Notice that this equation does not take the identities 3.116 into account. When the identities are considered the Jacobian of the above system becomes:

$$
\left[\begin{array}{ccc}
\mathrm{d} Q_{[13,14,15,16],[2,3,4,5]} & d Q_{[13,14,15,16],[2,3,4,6]} & d Q_{[13,14,15,16],[2,3,4,7]} \\
d Q_{[1,13,14,15,16],[1,2,3,4,5]} & d Q_{[1,13,14,15,16],[1,2,3,4,6]} & d Q_{[1,13,14,15,16],[1,2,3,4,7]} \\
d Q_{[8,13,14,15,16],[1,2,3,4,5]} & d Q_{[8,13,14,15,16],[1,2,3,4,6]} & d Q_{[8,13,14,15,16],[1,2,3,4,7]}
\end{array}\right]
$$

This Jacobian portends trouble: either the solution to the system 3.137, 3.138, and 3.139 . 
is trivial, or else these equations are not all independent. We may use Graßmann relations to show the latter. For the equations to be equivalent, the rank of this matrix must be one. The rank is one if and only if every $2 \times 2$ minor is identically zero. Start with the upper left $2 \times 2$ minor

$$
\begin{gathered}
d Q_{[13,14,15,16],[2,3,4,5]} d Q_{[1,13,14,15,16],[1,2,3,4,6]}- \\
d Q_{[13,14,15,16],[2,3,4,6]} d Q_{[1,13,14,15,16],[1,2,3,4,5]}
\end{gathered}
$$

In order to use Graßmann identities to show that 3.141 is identically zero, consider the matrix

$$
\left[\begin{array}{lllllllll}
Q_{1,1} & Q_{1,2} & Q_{1,3} & Q_{1,4} & Q_{1,5} & Q_{1,6} & Q_{1,7} & 1 & 0 \\
Q_{8,1} & Q_{8,2} & Q_{8,3} & Q_{8,4} & Q_{8,5} & Q_{8,6} & Q_{8,7} & 0 & 1 \\
Q_{13,1} & Q_{13,2} & Q_{13,3} & Q_{13,4} & Q_{13,5} & Q_{13,6} & Q_{13,7} & 0 & 0 \\
Q_{14,1} & Q_{14,2} & Q_{14,3} & Q_{14,4} & Q_{14,5} & Q_{14,6} & Q_{14,7} & 0 & 0 \\
Q_{15,1} & Q_{15,2} & Q_{15,3} & Q_{15,4} & Q_{15,5} & Q_{15,6} & Q_{15,7} & 0 & 0 \\
Q_{16,1} & Q_{16,2} & Q_{16,3} & Q_{16,4} & Q_{16,5} & Q_{16,6} & Q_{16,7} & 0 & 0
\end{array}\right]
$$

In the Graßmann notation with respect to this matrix, 3.141 is

$$
\pi_{2,3,4,5,8,9} \pi_{1,2,3,4,6,9}-\pi_{2,3,4,6,8,9} \pi_{1,2,3,4,5,9}
$$

The Graßmann relation beginning with these terms is

$$
\pi_{2,3,4,5,8,9} \pi_{1,2,3,4,6,9}-\pi_{2,3,4,6,8,9} \pi_{1,2,3,4,5,9}-\pi_{2,3,4,5,6,9} \pi_{1,2,3,4,8,9}=0
$$

Hence, the upper left $2 \times 2$ minor in equation 3.141 equals

$$
d Q_{[1,13,14,15,16],[2,3,4,5,6]} d Q_{[13,14,15,16],[1,2,3,4]}
$$

But the submatrix of $Q$ containing rows $[1,7,8,9,10,11,12,13,14,15,16]$ and columns $[2,3,4,5,6]$ is of rank four. So the minor $d Q_{[1,13,14,15,16],\{2,3,4,5,6]}$ is identically zero. Then the expression in 3.145 is identically zero, which forces the minor in 3.141 to be identically zero. Graßmann identities plus consistency conditions can be used to show that each $2 \times 2$ minor of the Jacobian in 3.140 is identically zero. The same holds for each of the eight sets of three equations. Amongst the two dozen conditions found, only eight are independent. The author prefers to work with the relations whose coefficients are of lowest degree in the data and uses the following solutions to eliminate eight of the $A_{i, j} \mathrm{~S}$ 


$$
\begin{aligned}
& A_{5,5}=-\frac{A_{6,5} \mathrm{dQ}_{[13,14,15,16],[2,3,4,6]}+A_{7,5} \mathrm{dQ}_{[13,14,15,16],[2,3,4,7]}}{\mathrm{d} Q_{[13,14,15,16],[2,3,4,5]}} \\
& A_{12,12}=-\frac{A_{10,12} \mathrm{dQ}_{[5,6,7,8],[10,13,14,15]}+A_{11,12} \mathrm{dQ}_{[5,6,7,8],[11,13,14,15]}}{\mathrm{dQ}_{[5,6,7,8],[12,13,14,15]}} \\
& A_{13,13}=-\frac{\mathrm{dQ}_{[5,6,7,8],[10,11,12,14]} A_{14,13}+A_{15,13} \mathrm{dQ}}{\mathrm{dQ}_{[5,6,6,7,8],[10,11,12,15]}} \\
& A_{8,8}=-\frac{A_{7,8} \mathrm{dQ}_{[13,14,15,16],[7,9,10,11]}+A_{0,8} \mathrm{dQ} Q_{[13,14,15,16],[6,9,10,11]}}{\mathrm{d} Q_{[13,14,15,16],[8,9,10,11]}} \\
& A_{9,9}=-\frac{A_{11,9} \mathrm{dQ}_{[13,14,15,16],[6,7,8,11]}+A_{10,9} \mathrm{~d} Q_{[13,14,15,16],[6,7,8,10]}}{\mathrm{d} Q_{[13,14,15,16],[6,7,8,9]}} \\
& A_{4,4}=-\frac{A_{2,4} \mathrm{dQ}_{[13,14,15,16],[2,5,6,7]}+A_{3,4} \mathrm{dQ}_{[13,14,15,16],[3,5,6,7]}}{\mathrm{dQ}_{[13,14,15,16],[4,5,6,7]}} \\
& A_{16,16}=-\frac{A_{15,16} \mathrm{dQ}_{[5,6,7,8],[1,2,3,15]}+A_{14,16} \mathrm{dQ}_{[5,6,7,8],[1,2,3,14]}}{\mathrm{dQ}_{[5,6,7,8],[1,2,3,16]}} \\
& A_{1,1}=-\frac{\mathrm{dQ}_{[5,6,7,8],[2,14,15,16]} A_{2,1}+\mathrm{dQ}_{[5,6,7,8],[3,14,15,16]} A_{3,1}}{\mathrm{~d} Q_{[5,6,7,8],[1,14,15,16]}}
\end{aligned}
$$

3.2.3.2.3 Hard Conditions We may now substitute the solutions in 3.146 and 3.116 back into the modified probabilities, (the data for the $2 \times 2$ subsystems which are the nonzero entries of the modified transition matrices $P_{i h}, P_{i o}, P_{h h}$, and $P_{h o}$ ). The eight $4 \times 4$ rank deficient submatrices which were not used to find the solutions in 3.146 and 3.116 may now be used to eliminate more of the $A_{i, j}$ s. As before eight $4 \times 4$ submatrices of rank two yield (at most) 32 independent conditions amongst the remaining $48 A_{i, j} \mathrm{~s}$.

The submatrices which have not yet been used to eliminate $A_{i, j}$ s are $Q 11_{l r}, Q 11_{t b}$, $Q 12_{r l}, Q 12_{b t}, Q 21_{l r}, Q 21_{b t}, Q 22_{b t}$, and $Q 22_{r l}$. Since the $3 \times 3$ minors of these equations cannot all be independent we need not bother simplifying all of them. These $3 \times 3$ minors are extremely cumbersome so the author generated a phantom and substituted its data into the minors in order to look for the simplest maximal spanning set of these minors. Some of these minors were much simpler than others. Recall that generically four conditions are required to force a $4 \times 4$ matrix to be of rank two. Although each rank deficient submatrix corresponds to four independent $3 \times 3$ minors, there may be dependencies between minors generated by different submatrices. As with the submatrices $Q 11_{r l}, Q 11_{b t}, Q 12_{l r}, Q 12_{t b}$, $Q 21_{r l}, Q 21_{t b}, Q 22_{t b}$, and $Q 22_{l r}$, which had only sixteen independent minors amongst them, the remaining eight submatrices correspond to only sixteen independent minors. 
Preferring the path of least resistance, the author chose to simplify as few of the general minors as possible, starting with the minors corresponding to rows $[1,2,3]$ and $[1,2,4]$ and columns $[1,2,3]$ for the submatrices $Q 12_{r l}, Q 21_{l r}, Q 22_{b t}$, and $Q 11_{t b}$. This choice of equations is not unique and was made simply because these equations looked simplest. The first step after writing down the equations is to eliminate their denominators. The next step is to collect appropriate terms in the equations. When a data set is substituted into the general equations the resulting equations have numerical coefficients and are referred to as numerical equations. The numerical equations have nearly as many terms as the general equations and many of their terms share the same coefficient. Once terms in the numerical equations with like coefficients are collected, the resulting equations have 1000 terms each. The arguments of like coefficients factor into a neat form (sometimes zero!). Collecting the general equations with respect to the minors of the data matrix, $Q$, yields 1000 term general equations. We may simplify each of these terms individually. The nontrivial terms which are independent of the data matrix $Q$ are either of the same form as 3.119 or of the form

$$
-d A_{[2,3,4],[1,3,4]} d A_{[1,2,3],[2,3,4]}+d A_{[1,2,3],[1,3,4]} d A_{[2,3,4],[2,3,4]}
$$

Referring to the matrix 3.120 , we can write 3.147 in Graßmann notation

$$
\pi_{1,3,4,5} \pi_{2,3,4,8}-\pi_{1,3,4,8} \pi_{2,3,4,5}
$$

which is the beginning of the Graßmann relation

$$
\pi_{1,3,4,5} \pi_{2,3,4,8}-\pi_{1,3,4,8} \pi_{2,3,4,5}-\pi_{3,4,5,8} \quad \pi_{1,2,3,4}=0
$$

From 3.149 we can make the substitution

$$
d A_{[2,3],[3,4]} d A_{[1,2,3,4],[1,2,3,4]}=-d A_{[2,3,4],[1,3,4]} d A_{[1,2,3],[2,3,4]}+d A_{[1,2,3],[1,3,4]} d A_{[2,3,4],[2,3,4]}
$$

Once substitutions like this are made, we can factor out a square of one of the following minors:

$$
d A_{[1,2,3,4],[1,2,3,4]}, d A_{5,6,7,8],[5,6,7,8]}, d A_{[0,10,11,12],\{9,10,11,12]}, \text { and } d A_{[13,14,15,16],[13,14,15,16]}
$$

as well as several generically nonzero minors of the data matrix. This step reduces the degree of the equations in $A_{i, j} \mathrm{~s}$ from thirteen to five and many of the terms in the equations are functions of minors of $A$. As long as the minors are written in the shorthand using the symbol $d A$, the identities in 3.116 are not recognized. So we must (have MAPLE) write out the minors and substitute the identities 3.116 into the equations. In both the numerical and general cases, the resulting equations have 256 terms, once they are collected with 
respect to the $A_{i, j}$ s. In the numerical case, the equations factor to have one quadratic term with 16 terms, another quadratic term with four terms and one linear term with only four terms. Generically, the relevant term is the linear one. Unfortunately, the author's general equations do not factor. The coefficients of each of the terms is a polynomial in minors of $Q$. The minors are expressed in the by-now familiar shorthand using the symbol $d Q$. As the Graßmann relations show, there are many ways of writing a polynomial in minors of a matrix. If MAPLE were able to handle equations of arbitrary size then the easiest thing to do would be to rewrite the equations without the $d Q$ notation and ask MAPLE to factor them. At present, that is not possible. So we must work.

Assuming that the general equations should factor just as their numerical counterparts do, we make good use of that knowledge. Consider the 64 combinations of variables which occur if we expand both quadratic terms in one of the equations. The coefficient of any one of these combinations is the desired linear term. Since this procedure was relatively easy, the author took the coefficient of each one of the 64 combinations and got 64 different linear terms. Fortunately, they are all equivalent. Although tedious, it is just as ' straightforward to show that these relations are equivalent as it is to show that the relations 3.137, 3.138, and 3.139 are equivalent. Once again, the author prefers to work with the equations which have the coefficients of lowest degree in the data. Two of the identities are shown below 


$$
\begin{aligned}
& 0=\left(\mathrm{dQ}_{[5,13,14,18,16],[1,2,3,4,8]} \mathrm{d} \mathrm{Q}_{[0,13,14,15,16],(8,9,10,11,12]}-\right. \\
& \left.\mathrm{dQ}_{[6,13,14,15,16],(1,2,3,4,8]} \mathrm{d} Q_{[8,13,14,18,16],(8,9,10,11,12]}\right) A_{8,6}+ \\
& \left(\mathrm{dQ}_{[3,13,14,16,16],(1,2,3,4,7]} \mathrm{d} \mathrm{Q}_{[6,13,14,15,16],(8,9,10,11,12]}{ }^{-}\right. \\
& d Q_{[6,13,14,15,16],(1,2,3,4,7]} d Q_{[5,13,14,18,16],(8,9,10,11,12]}-
\end{aligned}
$$

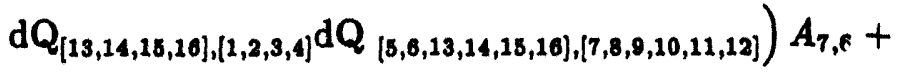

$$
\begin{aligned}
& \left(-d Q_{[13,14,15,16],(1,2,3,4]} d Q_{[5,6,13,14,15,16],[5,8,9,10,11,12]}{ }^{+}\right. \\
& d Q_{[5,13,14,16,16],(1,2,3,4,5]} d Q_{[6,13,14,15,16],(8,9,10,11,12]}- \\
& \left.\mathrm{dQ}_{[6,13,14,15,16],[1,2,3,4,5]} \mathrm{d} \mathrm{Q}_{[5,13,14,15,16],(8,9,10,11,12]}\right) A_{5,6}+ \\
& \left(-d Q_{[13,14,15,16],(1,2,3,4]} d Q_{[5,6,13,14,15,16],[6,8,9,10,11,12]}-\right. \\
& d Q_{[6,13,14,15,16],[1,2,3,4,6]} d Q_{[5,13,14,15,16],(8,9,10,11,12]}+ \\
& \left.\mathrm{dQ}_{[6,13,14,15,16],[8,9,10,11,12]} \mathrm{dQ}_{[5,13,14,15,16],(1,2,3,4,6]}\right) A_{6,6}
\end{aligned}
$$

and

$$
\begin{aligned}
& 0=\left(-d Q_{[5,13,14,15,16],[1,2,3,4,8]} d Q_{[7,13,14,15,16],[8,9,10,11,12]}+\right. \\
& \left.\mathrm{d} \mathrm{Q}_{[5,13,14,15,16],(8,9,10,11,12]} \mathrm{d} \mathrm{Q}_{[7,13,14,15,16],[1,2,3,4,8]}\right) A_{8,6}+ \\
& \left(-d Q_{[5,13,14,15,16],(1,2,3,4,7]} d Q_{[7,13,14,15,16],(8,9,10,11,12]}+\right. \\
& \mathrm{dQ}_{[7,13,14,15,16],[1,2,3,4,7]} \mathrm{d} \mathrm{Q}_{[5,13,14,15,16],[8,9,10,11,12]}+ \\
& \left.\mathrm{dQ}_{[5,7,13,14,15,16],[7,8,9,10,11,12]} \mathrm{dQ}_{[13,14,15,16],[1,2,3,4]}\right) A_{7,6}+ \\
& \left(-d Q_{[5,13,14,15,16],(1,2,3,4,5]} d Q_{[7,13,14,15,16],(8,9,10,11,12]^{+}}\right. \\
& \mathrm{dQ}_{[5,13,14,15,16],(8,9,10,11,12]} \mathrm{d} \mathrm{Q}_{[7,13,14,15,16],[1,2,3,4,5]}+ \\
& \left.\mathrm{dQ}_{[5,7,13,14,15,16],[5,8,9,10,11,12]} \mathrm{dQ}_{[13,14,15,16],[1,2,3,4]}\right) A_{5,6}+ \\
& \left(-d Q_{[7,13,14,15,16],[8,9,10,11,12]} \mathrm{dQ}_{[5,13,14,15,16],[1,2,3,4,6]}+\right. \\
& \mathrm{dQ}_{[5,13,14,15,16],[8,9,10,11,12]} \mathrm{dQ} \mathrm{Q}_{[7,13,14,15,16],\{1,2,3,4,6]}+ \\
& \left.\mathrm{dQ}_{[5,7,13,14,15,16],[6,8,9,10,11,12]} \mathrm{dQ}_{[13,14,15,16],[1,2,3,4]}\right) A_{6,6}
\end{aligned}
$$

These equations are independent and yield the following solutions for $A_{8,6}$ and $A_{5,6}$. 
88

$$
\begin{aligned}
& A_{8,6}=\left(-\left(\mathrm{d} Q_{[6,7,13,14,28,16],[1,2,3,4,5,6]} \quad \mathrm{d} Q_{[8,13,14,15,10],(8,9,10,11,12]}+\right.\right. \\
& \mathrm{dQ}_{[7,13,14,16,16],(1,2,3,4,0]} \quad \mathrm{dQ}_{[5,6,13,14,15,16],[6,8,9,10,11,12]}- \\
& \mathrm{dQ}_{[5,7,13,14,15,16],[8,8,0,10,11,12]} \mathrm{dQ}_{[0,13,14,15,16],[1,2,3,4,0]}+ \\
& \mathrm{d} Q_{[5,7,13,14,15,16],[6,8,8,10,11,12]} \quad \mathrm{dQ}_{(6,13,14,16,16],[1,2,3,4,5]} \quad- \\
& \mathrm{dQ}_{[6,13,14,16,16],[8,9,10,11,12]} \mathrm{dQ} \mathrm{Q}_{[5,7,13,14,16,16],[1,2,3,4,3,6]} \\
& d Q_{[7,13,14,15,16],\{1,2,3,4,5]} \quad \mathrm{dQ}_{[5,6,13,14,15,16),(0,8,9,10,11,12]} \\
& \mathrm{dQ}_{[6,7,13,14,15,16],[6,8,8,10,11,12]} \quad \mathrm{d} Q_{[5,13,14,15,16],[1,2,3,4,5]}+ \\
& \mathrm{dQ}_{[7,13,14,15,16],(8,9,10,11,12]} \quad \mathrm{dQ}_{[5,0,13,14,15,16],(1,2,3,1,5,6]}+ \\
& \mathrm{dQ}_{[6,7,13,14,15,16],[5,8,9,10,11,12]} \quad \mathrm{d} Q_{[5,13,14,15,16],[1,2,3,4,6]}+ \\
& \left.\mathrm{d} Q_{[5,6,7,13,14,15,16],[5,6,8,9,10,11,12]} \quad \mathrm{d} Q_{[13,14,15,16],[1,2,3,4]}\right) A_{6,6}- \\
& \left(\mathrm{dQ}_{[0,7,13,14,15,16],[1,2,3,4,5,7]} \quad \mathrm{dQ}_{[5,13,14,15,16],[8,8,10,11,12]} \quad-\right. \\
& \mathrm{dQ}_{[5,7,13,14,15,16],[5,8,9,10,11,12]} \quad \mathrm{dQ}_{[6,13,14,15,16],[1,2,3,4,7]} \quad- \\
& \mathrm{dQ}_{[7,13,14,15,16],[1,2,3,4,5]} \quad \mathrm{dQ}_{[5,6,13,14,15,16],[7,8,9,10,11,12]}+ \\
& \mathrm{dQ}_{[7,13,14,15,16],(1,2,3,4,7]} \mathrm{dQ}[5,6,13,14,15,16],[5,8,9,10,11,12]+ \\
& \mathrm{dQ}_{[5,7,13,14,15,16],[7,8,9,10,11,12]} \mathrm{d} Q_{[6,13,14,15,16],[1,2,3,4,5]}+
\end{aligned}
$$

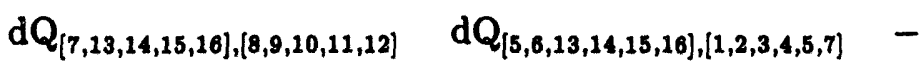

$$
\begin{aligned}
& \mathrm{dQ}_{[6,7,13,14,15,16],[7,8,9,10,11,12]} \quad \mathrm{dQ}_{[5,13,14,15,16],[1,2,3,4,3]} \\
& \mathrm{dQ} Q_{[6,13,14,15,16],\{8,9,10,11,12]} \quad \mathrm{dQ}_{[5,7,13,14,15,16],[1,2,3,4,5,7]}+ \\
& d Q_{[6,7,13,14,15,16],[5,8,9,10,11,12]} \quad \mathrm{dQ}_{[5,13,14,15,16],[1,2,3,4,7]}+ \\
& \left.\left.\mathrm{dQ}_{[13,14,15,16],[1,2,3,4]} \quad \mathrm{dQ}_{[5,6,7,13,14,15,16],[5,7,8,9,10,11,12]}\right) A_{7,6}\right) / \\
& \left(\mathrm{d} \mathrm{Q}_{[6,7,13,14,15,16],[1,2,3,4,5,8]} \quad \mathrm{d} \mathrm{Q}_{[5,13,14,15,16],[8,9,10,11,12]}\right. \text { - } \\
& \mathrm{dQ}_{[6,13,14,15,16],[1,2,3,4,8]} \mathrm{d} \mathrm{Q}_{[5,7,13,14,15,16],[5,8,9,10,11,12]}+ \\
& \mathrm{d} \mathrm{Q}_{[7,13,14,15,16],[1,2,3,4,8]} \mathrm{d} \mathrm{Q}_{[5,6,13,14,15,16],[5,8,9,10,11,12]} \quad- \\
& \mathrm{d} \mathrm{Q}_{[6,13,14,15,16],[8,9,10,11,12]} \quad \mathrm{d} \mathrm{Q}_{[5,7,13,14,15,16],[1,2,3,4,5,8]}+ \\
& \mathrm{dQ}_{[7,13,14,15,16],[8,9,10,11,12]} \quad \mathrm{dQ}_{[5,6,13,14,15,16],[1,2,3,4,5,8]}+ \\
& \left.\mathrm{dQ}_{[6,7,13,14,15,16],[5,8,8,10,11,12]} \quad \mathrm{dQ}[5,13,14,15,16],(1,2,3,4,8]\right)
\end{aligned}
$$




$$
\begin{aligned}
& A_{5,0}=\left(\left(-\mathrm{dQ}_{[7,13,14,18,16],(1,2,3,4,8]} d Q_{[5,8,18,14,18,16],(6,8,0,10,11,12]}+\right.\right. \\
& d Q_{[6,13,14,18,16],(1,2,3,4,8]} d Q_{[8,7,13,14,16,10],(0,8,9,10,11,12]} \quad \text { - } \\
& d Q_{[0,7,13,14,15,10],[6,8,0,10,11,12]} d Q_{[8,13,14,15,10],(1,2,3,4,8]} \quad \text { - } \\
& d Q_{[0,7,13,14,15,16],\{1,2,3,4,0,8]} d Q_{[3,13,14,13,16],[8,0,10,11,12]}+ \\
& d Q_{[5,7,13,14,15,16],(1,2,3,4,6,8]} d Q_{[0,13,14,15,16],(8,9,10,11,12]} \quad \text { - } \\
& \left.\mathrm{dQ}_{[3,0,13,14,15,16],[1,2,3,4,6,8]} \quad d Q_{[7,13,14,18,16],(8,9,10,11,12]}\right) A_{6,6}+ \\
& \left(d Q_{[0,13,14,15,16],[1,2,3,4,8]} d Q_{[8,7,13,14,15,16],(7,8,9,10,11,12]}\right. \text { - } \\
& d Q_{[7,13,14,15,16],(1,2,3,4,8]} d Q_{[5,6,13,14,15,16],(7,8,9,10,11,12]} \quad \text { - } \\
& d Q_{[5,13,14,15,10],[1,2,3,4,8]} d Q_{[0,7,13,14,16,16],[7,8,9,10,11,12]} \quad \text { - } \\
& \mathrm{dQ}_{[5,6,13,14,15,16],(1,2,3,4,7,8]} d \mathrm{Q}_{[7,13,14,15,16],(8,9,10,11,12]} \text { - } \\
& \mathrm{dQ}_{[6,7,13,14,15,16],(1,2,3,4,7,8]} d \mathrm{Q}_{[5,13,14,15,16],(8,9,10,11,12]}+ \\
& \left.\left.\mathrm{dQ}_{[5,7,13,14,15,10],(1,2,3,4,7,8]} \quad \mathrm{dQ}_{[6,13,14,15,16],(8,9,10,11,12]}\right) A_{7,6}\right) / \\
& \left(d Q_{[0,7,13,14,15,16],[1,2,3,4,5,8]} d Q_{[5,13,14,15,16],[8,9,10,11,12]}\right. \text { - } \\
& d Q_{[6,13,14,15,16],[1,2,3,4,8]} d Q_{[5,7,13,14,15,16],(5,8,9,10,11,12]}+ \\
& d Q_{[7,13,14,15,16],(2,2,3,4,8]} d Q_{[5,6,13,14,15,16],[5,8,9,10,11,12]} \\
& \mathrm{dQ}_{[0,13,14,15,16],\{8,9,10,11,12]} \mathrm{d} \mathrm{Q}_{[5,7,13,14,15,16],\{1,2,3,4,5,8]}+ \\
& \mathrm{dQ}_{[7,13,14,15,16],(8,9,10,11,12]} d \mathrm{Q}_{[5,6,13,14,15,16],(1,2,3,4,5,8]}+ \\
& \left.\mathrm{dQ}_{[6,7,13,14,15,16],[5,8,9,10,11,12]} \quad \mathrm{dQ}_{[5,13,14,15,16],\{1,2,3,4,8]}\right)
\end{aligned}
$$

3.2.3.2.4 Very Hard Conditions The only rank deficient submatrices we have not yet accounted for are $Q 22_{r l}, Q 11_{l r}, Q 21_{b t}$, and $Q 12_{t b}$. The author chose to simplify the simplest minors generated by the phantom, those from columns $[1,2,4]$ and both sets of rows $[1,2,4]$ and $[1,3,4]$. The equations obtained by subtituting 3.153 and its counterparts into these remaining minors are polynomials in the remaining $A_{i, j} \mathrm{~s}$ and with coefficients which are large polynomials in the minors of $Q$. In preliminary work with a phantom each of these equations was a quintic in the $A_{i, j}$ s and became the product of a 32 term quartic and a linear term after factorization. Upon substituting the phantom's values for the $A_{i, j} s$ into the minors, the relevant terms turned out to be the linear terms. Unfortunately, the 
80

general versions of these minors did not factor, presumably because the coefficients were written in the " $d Q$ " notation. As with the hard conditions, the author assumed that the coefficient of any of the 32 terms in the quartic is the desired linear term.

Because their coefficients are so cumbersome, only a caricature of one of these identities is shown below

$$
\begin{aligned}
& \left(c_{5}\left(c_{4} a_{8}-b_{2} c_{8}+b_{6} c_{8}\right) d_{1}-\mathrm{d} Q_{[8,0,7,8],(13,14,15,10]} \mathrm{d} Q_{[8,6,7,8],[0,10,11,12]}\right. \\
& \left(\mathrm{d} \mathrm{Q}_{[13,14,13,10],(8,9,10,12]} a_{1}-a_{4} \mathrm{dQ} \mathrm{Q}_{[13,14,15,16],(8,9,10,11]}\right) \\
& \left(b_{1} d Q_{[11,13,14,15,16],(6,7,8,9,11]}-b_{3} d Q_{[10,13,14,15,10],(0,7,8,9,11]}\right) \\
& -d Q_{[13,14,13,16],(6,7,8,9]} d Q_{[3,0,7,8],(9,10,11,12]} a_{6}\left(a_{3} d Q_{[13,14,15,16],(8,9,10,11]}\right. \\
& \left.+\mathrm{d} Q_{[3,6,7,8,10],(10,13,14,18,16]} a_{2} d Q_{[13,14,15,10],(8,9,11,12]}\right) \\
& +d Q_{[13,14,18,16],(6,7,8,9]} d Q_{[5,6,7,8],[9,10,11,12]} a_{1}\left(a_{7} d Q_{[10,13,14,13,16],[8,9,10,11,12]}\right. \\
& \left.-a_{5} \mathrm{~d} Q_{[11,13,14,15,16],(8,9,10,11,12]}\right) \\
& +\mathrm{d} Q_{[13,14,15,16],[6,7,8,9]} \mathrm{d} Q_{[5,0,7,8],[0,10,11,12]}\left(a_{7} \mathrm{~d} Q_{[13,14,15,16],(8,9,10,11]}\right. \\
& \left.+\mathrm{dQ}_{[5,6,7,8,11],(10,13,14,18,16]} a_{2} \mathrm{~d} Q_{[13,14,15,10],(8,9,11,12]}\right) a 9 \\
& -d Q_{[5,6,7,8],(8,10,11,12]}\left(b_{4} d Q_{[13,14,15,16],[8,9,11,12]}+b_{3} d Q_{[13,14,15,16],(8,9,10,11]}\right) c_{5} a_{4} \\
& +\mathrm{d} Q_{[5,6,7,8],\{9,10,11,12]}\left(c_{1} a_{2} \mathrm{~d} Q_{[13,14,15,16],[6,7,8,9]} a_{3}-\mathrm{d} Q_{[5,6,7,8],(13,14,15,16]} c_{2} a_{2} a_{4}\right. \\
& -\mathrm{dQ}_{[13,14,15,16],(6,7,8,9]} \mathrm{dQ} \mathrm{Q}_{[13,14,15,16],(8,9,10,11]} a_{10} a_{3}+\mathrm{d} Q_{[13,14,15,16],(8,9,10,12]} b_{5} a_{1} c_{5} \\
& +\mathrm{dQ}_{[13,14,15,16],[8,9,10,12]} b_{4} c_{5} a_{3}+\mathrm{dQ} Q_{[13,14,15,16],(6,7,8,9]} a_{1} \mathrm{~d} Q_{[13,14,15,16],(8,9,11,12]} a_{10} \\
& \left.\left.+\mathrm{d} \mathrm{Q}_{[3,6,7,8],[13,14,15,16]} c_{3} a_{2} a_{6}-\mathrm{d} Q_{[5,0,7,8\},[13,14,15,10]} c_{7} a 9 a_{2}\right)\right) A[10,11] \\
& +\left(-c_{9}\left(c_{4} a_{8}-b_{2} c_{6}+b_{6} c_{8}\right) d_{1}-\mathrm{d} Q_{[13,14,15,16],(6,7,8,9]} \mathrm{dQ}_{[5,6,7,8],[0,10,11,12]}\right. \\
& \left(b_{5} d Q_{[13,14,15,16],[8,9,10,11]}+d Q_{[13,14,15,16],(8,9,11,12]} a_{2}\right) \\
& \left(-d Q_{[5,6,7,8,11],(9,13,14,15,16]} a 9+d Q_{[3,6,7,8,10],[9,13,14,13,16]} a_{6}\right) \\
& +\mathrm{dQ}_{[5,6,7,8],\{8,10,11,12]}\left(b_{4} \mathrm{~d} \mathrm{Q}_{[13,14,15,16],[8,9,11,12]}+b_{5} \mathrm{~d} \mathrm{Q}_{[13,14,15,16],(8,9,10,11]}\right) c_{9} a_{4} \\
& -\mathrm{d} Q_{[5,6,7,8],(9,10,11,12]}\left(-\mathrm{d} Q_{[13,14,15,16],(6,7,8,9]} \mathrm{d} Q_{[13,14,15,10],(8,9,10,11]} a_{11} a_{3}\right. \\
& +\mathrm{d} Q_{[13,14,15,16],[6,7,8,9]} a_{1} \mathrm{dQ}_{[13,14,15,16],[8,9,11,12]} a_{11}-c_{10} a_{2} \mathrm{~d} Q_{[13,14,16,16],(6,7,8,9]} a_{3} \\
& +\mathrm{dQ}_{[13,14,15,16],(8,9,10,12]} b_{4} c_{9} a_{3}-c_{10} b_{5} \mathrm{dQ} Q_{[13,14,15,16],(6,7,8,9]} a_{1} \\
& \left.\left.+\mathrm{d} Q_{[13,14,15,16),(8,9,10,12]} b_{5} a_{1} c_{9}\right)\right) A[9,11]
\end{aligned}
$$


91

$$
\begin{aligned}
& +\left(d Q _ { [ 1 8 , 1 4 , 1 8 , 1 0 ] , ( 6 , 7 , 8 , 9 ] } d Q _ { [ 8 , 0 , 7 , 8 ] , ( 0 , 1 0 , 1 1 , 1 2 ] } \left(-d Q_{[11,13,14,15,10],(8,9,10,11,12]}\right.\right. \\
& \left(-b_{1} d Q_{[s, 0,7,8],\{13,14,18,16]}+d Q_{[8,0,7,8,10],[12,13,14,15,10]} a_{2}\right) \\
& \left.-\left(-b_{3} d Q_{[8,6,7,8],(13,14,18,16]}+d Q_{[8,8,7,8,11],[12,13,16,18,16]} a_{2}\right) d Q_{[10,13,14,18,16],(8,9,10,11,12]}\right) a_{3} \\
& +d Q_{[13,14,18,16],(6,7,8,9]} d Q_{[5,0,7,8],(6,10,11,12]}\left(-d Q_{[13,14,18,16],[8,9,11,12]} b_{3} d Q_{[5,0,7,8],[13,14,18,16]}\right. \\
& +d Q_{[8,6,7,8,11],(12,13,14,18,16]} b_{3} d Q_{[13,14,16,16],(8,9,10,11]} \\
& \left.+\mathrm{dQ}_{[5,0,7,8,11],(12,13,14,15,10]} \mathrm{d} \mathrm{Q}_{[13,14,15,10],(8,9,11,12]} a_{2}\right) a 9 \\
& -d Q_{[13,14,18,10],[6,7,8,0]} d Q_{[5,6,7,8],[9,10,11,12]}\left(-d Q_{[13,14,18,16],(8,9,11,12]} b_{1} d Q_{[8,6,7,8],[13,14,18,10]}\right. \\
& +d Q_{[8,6,7,8,10],\{12,13,14,13,10]} b_{8} d Q_{[13,14,15,16],\{8,9,10,11]} \\
& \left.+\mathrm{d} Q_{[5,6,7,8,10],(12,13,14,15,10]} \mathrm{d} Q_{[13,14,15,16],(8,9,11,12]} a_{2}\right) a_{6} \\
& -d Q_{[3,0,7,8],(0,10,11,12]}\left(b_{4} d Q_{[13,14,18,16],(8,9,11,12]}+b_{5} d Q_{[13,14,15,16],(8,9,10,11]}\right) c_{11} a_{4} \\
& +d Q_{[13,14,15,16],(6,7,8,9]} d Q_{[5,6,7,8],(0,10,11,12]} \\
& \left(d Q_{[5,6,7,8,11],(12,13,14,15,16]} d Q_{[5,6,7,8,10,11],(10,12,13,14,15,16]} d Q_{[9,10,13,14,13,16],(3,0,7,8,9,12]}\right. \\
& +d Q_{[5,6,7,8,10],\{12,13,14,15,16]} d Q_{[11,13,14,15,16],[5,6,7,8,12]} d Q_{[5,6,7,9,9,10,11],\{9,10,12,13,14,18,16]} \\
& -d Q_{[3,6,7,8,11],(12,13,14,15,16]} d Q_{[10,13,14,15,16],(5,6,7,8,12]} d Q_{[5,6,7,8,9,10,11],[0,10,12,13,14,13,16]} \\
& +d Q_{[5,6,7,8,10],(12,13,14,15,16]} d Q_{[5,6,7,8,10,11],(0,12,13,14,15,10]} d Q_{[9,11,13,14,15,16], 15,0,7,8,10,12]} \\
& -d Q_{[5,6,7,8,10],[12,13,14,15,10]} d Q_{[5,6,7,8,10,11],(10,12,13,14,15,16]} d Q_{[9,11,13,14,15,16],(3,6,7,8,9,12]} \\
& -d Q_{[5,6,7,8,11],(12,13,14,15,16]} d Q_{[5,6,7,8,10,11],(0,12,13,16,15,16]} d Q_{[9,10,13,14,13,16],[5,6,7,8,10,12]} \\
& -d Q_{[10,11,13,14,15,16],[5,6,7,8,10,12]} d Q_{[5,0,7,8,10,11],[9,12,13,14,15,16]} d Q_{[5,6,7,8,9],[12,13,14,15,16]} \\
& \left.+d Q_{[10,11,13,14,15,16],[5,6,7,8,9,12]} \mathrm{dQ}_{[5,6,7,8,10,11],[10,12,13,14,15,16]} \mathrm{d} \mathrm{Q}_{[5,6,7,8,9],[12,13,14,18,16]}\right) \\
& \left(-\mathrm{d} \mathrm{Q}_{[13,14,15,16],(8,9,11,12]} a_{1}+\mathrm{d} \mathrm{Q}_{[13,14,15,16],(8,9,10,11]} a_{3}\right) \\
& +d Q_{[5,6,7,8],[13,14,15,16]} d Q_{[5,6,7,8],\{9,10,11,12]} \\
& \left(-a_{4} \mathrm{dQ}_{[13,14,15,10],[8,9,11,12]}+\mathrm{d} \mathrm{Q}_{[13,14,15,16],(8,9,10,12]} a_{3}\right) \\
& \left(b_{1} \mathrm{dQ} Q_{[11,13,14,15,16],[6,7,8,9,11]}-b_{3} \mathrm{~d} \mathrm{Q}_{[10,13,14,15,16],[6,7,8,9,11]}\right) \\
& +c_{8} c_{11} d_{1} b_{6}+c_{4} c_{11} a_{8} d_{1}-c_{6} c_{11} d_{1} b_{2} \\
& +c_{11}\left(b_{4} a_{3}+a_{1} b_{5}\right) \mathrm{dQ}_{[13,14,15,16],(8,9,10,12]} \mathrm{dQ}_{[5,6,7,8],[9,10,11,12]} \\
& +b_{5}\left(-a 9 c_{7}-a_{4} c_{2}+c_{3} a_{6}\right) \mathrm{dQ}_{[5,6,7,8],(13,14,15,16]} \mathrm{d} \mathrm{Q}_{[8,6,7,8],\{9,10,11,12]} \\
& \left.+\mathrm{d} Q_{[5,6,7,8],(9,10,11,12]} c_{12} b_{5} \mathrm{dQ}_{[13,14,13,16),[6,7,8,9]} a_{1}\right) A[12,11]
\end{aligned}
$$



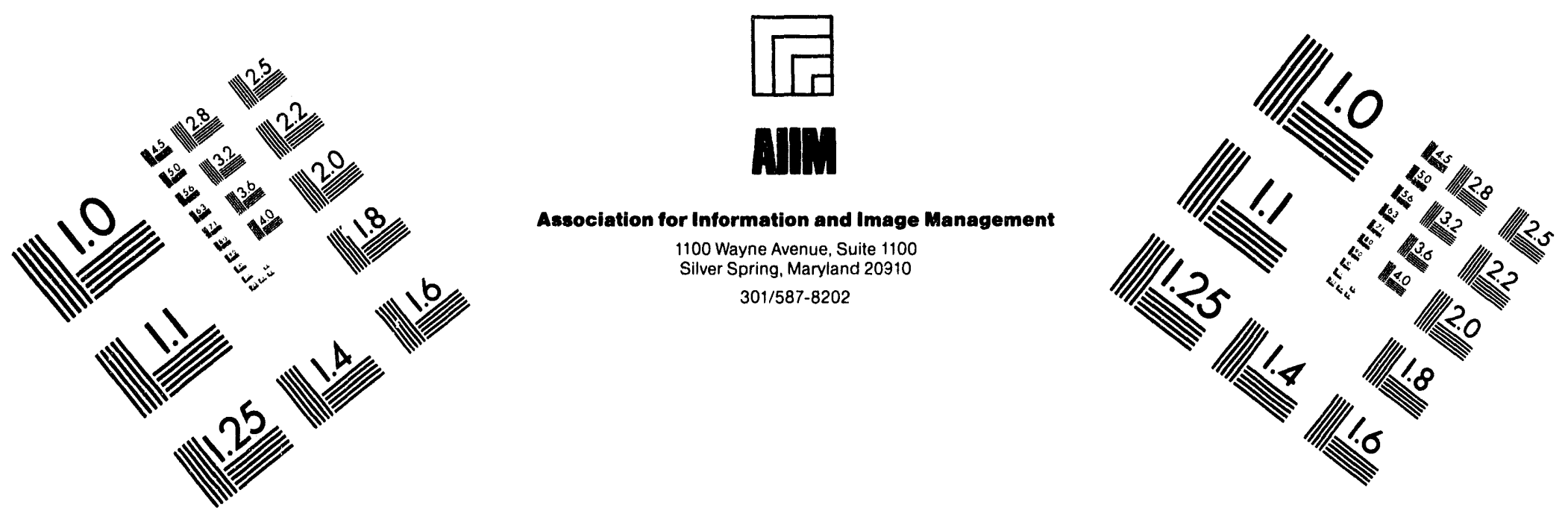

\section{Centimeter}

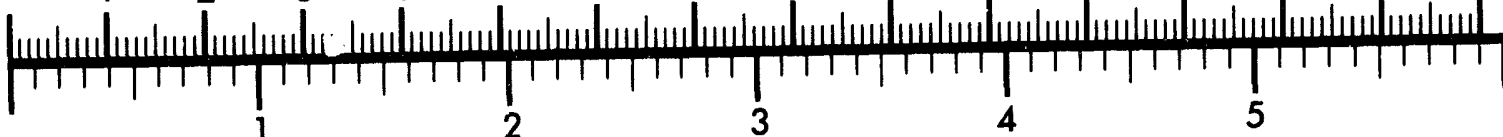
Inches
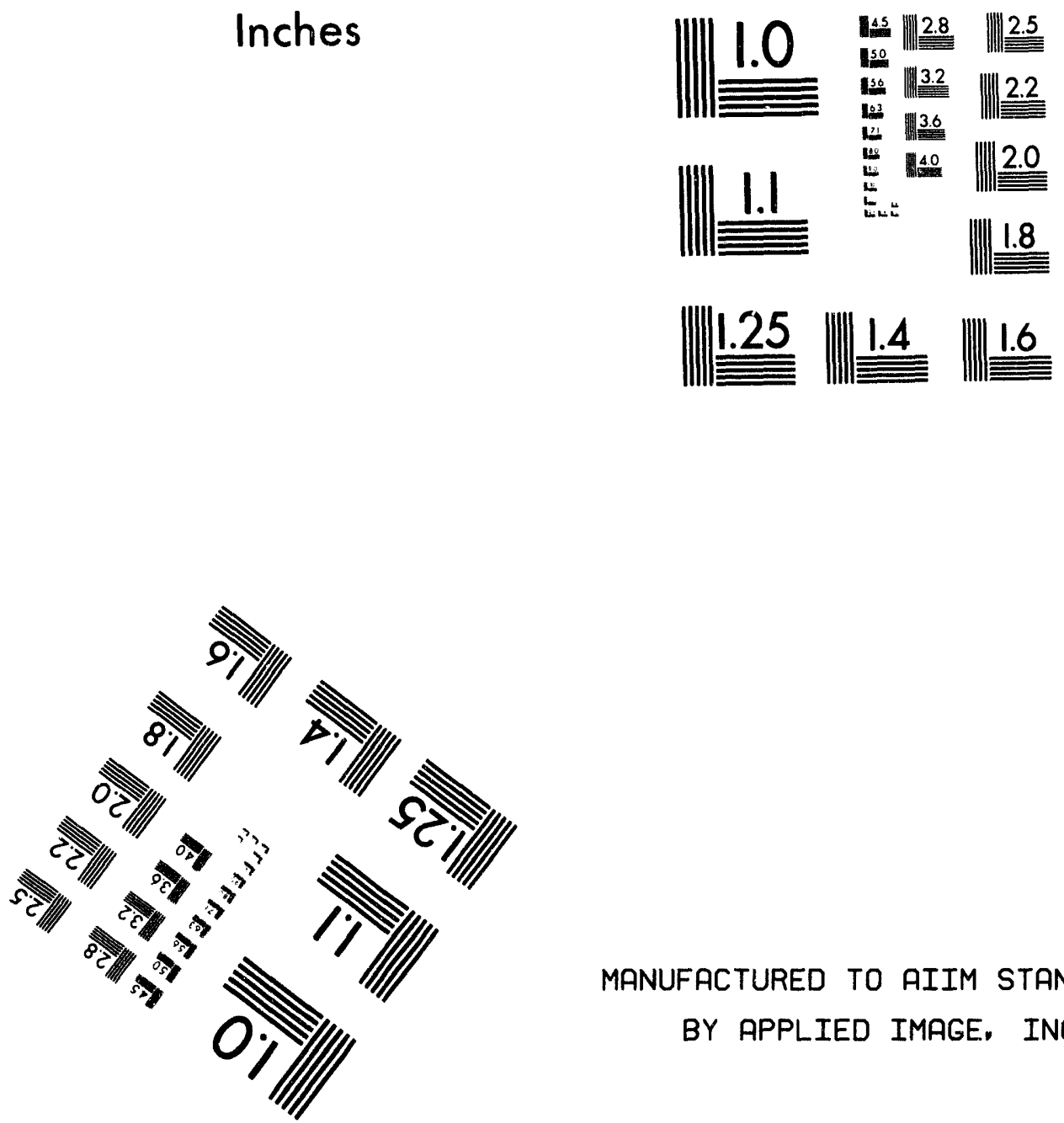

MANUFACTURED TO AIIM STANDARDS

BY APPLIED IMAGE, INC.

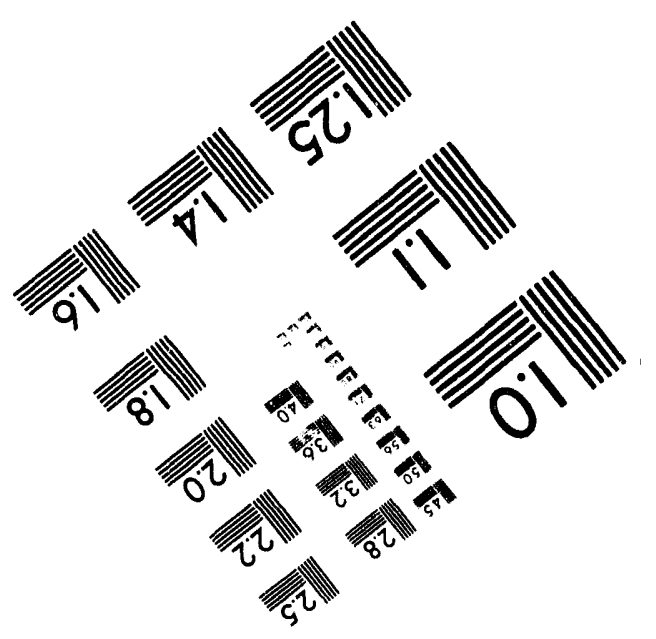



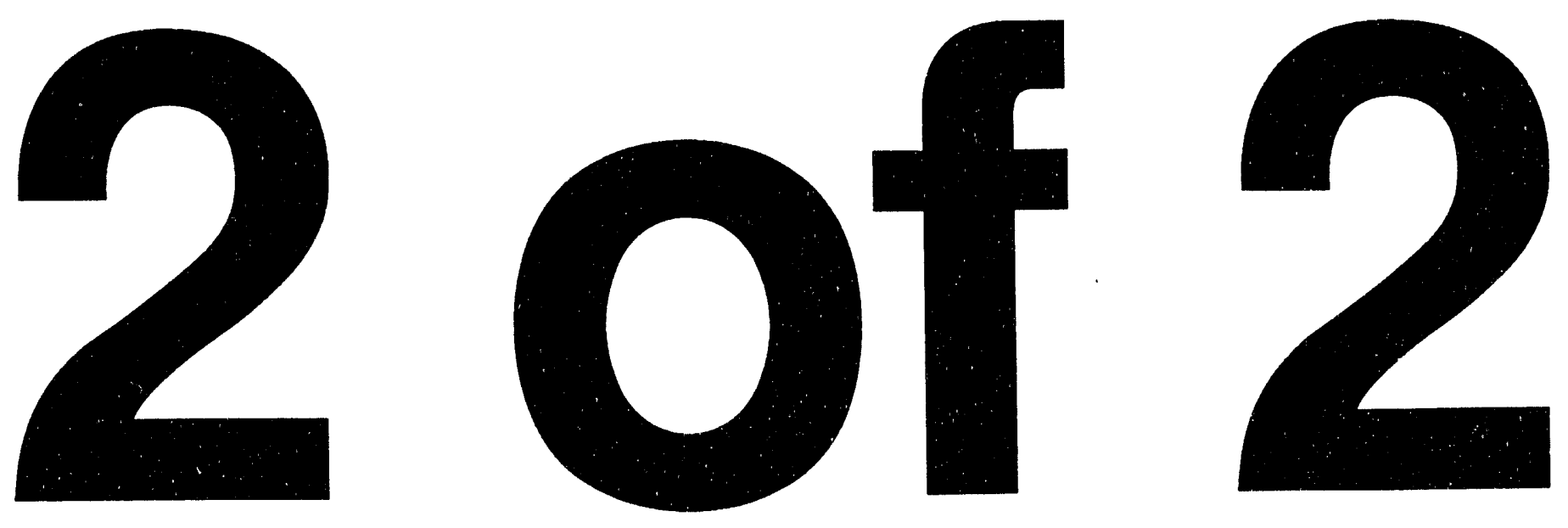


$$
\begin{aligned}
& +\left(-\mathrm{dQ}_{[5,6,7,8],[9,10,11,12]}\left(-c_{14} a 9+c_{15} a_{6}\right)\right. \\
& \left(b_{5} \mathrm{dQ}_{[13,14,15,16],[8,9,10,11]}+\mathrm{dQ}_{[13,14,15,16],[8,9,11,12]} a_{2}\right) \\
& +d Q_{[5,6,7,8],[13,14,15,16]} d Q_{[5,6,7,8],[9,10,11,12]} \\
& \left(-\mathrm{dQ}_{[13,14,15,16],[8,9,11,12]} a_{1}+\mathrm{d} Q_{[13,14,15,16],[8,9,10,11]} a_{3}\right) \\
& \left(b_{1} \mathrm{dQ}_{[11,13,14,15,16],[6,7,8,9,11]}-b_{3} \mathrm{dQ}_{[10,13,14,15,16],[6,7,8,9,11]}\right) \\
& -c_{6} c_{13} d_{1} b_{2}+\mathrm{dQ}_{[5,6,7,8],[9,10,11,12]} \mathrm{dQ}_{[13,14,15,16],[8,9,10,12]} b_{5} a_{1} c_{13} \\
& -\mathrm{dQ}_{[5,6,7,8],[8,10,11,12]}\left(b_{4} \mathrm{dQ}_{[13,14,15,16],[8,9,11,12]}+b_{5} \mathrm{dQ}_{[13,14,15,16],[8,9,10,11]}\right) c_{13} a_{4} \\
& +\mathrm{dQ}_{[5,6,7,8],[9,10,11,12]} \mathrm{dQ}_{[13,14,15,16],[8,9,10,12]} b_{4} a_{3} c_{13} \\
& +c_{4} c_{13} a_{8} d_{1}+c_{8} c_{13} d_{1} b_{6}+\mathrm{dQ}_{[13,14,15,16],[6,7,8,9]} \mathrm{dQ}_{[5,6,7,8],[9,10,11,12]} \\
& \left(-d Q_{[5,6,7,8,10],[11,13,14,15,16]} d Q_{[5,6,7,8,10,11],[10,12,13,14,15,16]} d Q_{[9,11,13,14,15,16],[5,6,7,8,9,12]}\right. \\
& -\mathrm{dQ}_{[10,11,13,14,15,16],[5,6,7,8,10,12]} \mathrm{dQ} \mathrm{Q}_{[5,6,7,8,9,11],[9,12,13,14,15,16]} \mathrm{d} \mathrm{Q}_{[5,6,7,8,10],[11,13,14,15,16]} \\
& +\mathrm{dQ}_{[10,11,13,14,15,16],[5,6,7,8,10,12]} \mathrm{d} \mathrm{Q}_{[5,6,7,8,9,10],[9,12,13,14,15,16]} \mathrm{d} \mathrm{Q}_{[5,6,7,8,11],[11,13,14,15,16]} \\
& -d Q_{[5,6,7,8,9,10,11],[9,10,12,13,14,15,16]} \mathrm{dQ}_{[10,13,14,15,16],[5,6,7,8,12]} \mathrm{dQ}_{[5,6,7,8,11],[11,13,14,15,16]} \\
& +\mathrm{dQ}_{[5,6,7,8,10],[11,13,14,15,16]} \mathrm{d} \mathrm{Q}_{[11,13,14,15,16],[5,6,7,8,12]} \mathrm{d} \mathrm{Q}_{[5,6,7,8,9,10,11],[9,10,12,13,14,15,16]} \\
& +d Q_{[10,11,13,14,15,16],\{5,6,7,8,9,12]} d Q_{[5,6,7,8,9,11],[10,12,13,14,15,16]} \mathrm{dQ}_{[5,6,7,8,10],[11,13,14,15,16]} \\
& -\mathrm{dQ}_{[5,6,7,8,9,10],[10,12,13,14,15,16]} \mathrm{dQ}_{[5,6,7,8,11],[11,13,14,15,16]} \mathrm{dQ}_{[10,11,13,14,15,16],[5,6,7,8,9,12]} \\
& -d Q_{[9,10,13,14,15,16],(5,6,7,8,10,12]} d Q_{[5,6,7,8,10,11],[9,12,13,14,15,16]} d Q_{[5,6,7,8,11],[11,13,14,15,16]} \\
& +\mathrm{dQ}_{[9,10,13,14,15,16],[5,6,7,8,9,12]} \mathrm{d} \mathrm{Q}_{[5,6,7,8,10,11],[10,12,13,14,15,16]} \mathrm{dQ}_{[5,6,7,8,11],[11,13,14,15,16]} \\
& +d Q_{[5,6,7,8,10],\{11,13,14,15,16]} d Q_{[5,6,7,8,10,11],[9,12,13,14,15,16]} \mathrm{dQ}_{[9,11,13,14,15,16],[5,6,7,8,10,12]} \\
& \left.+d Q_{[9,10,11,13,14,15,16],[5,6,7,8,9,10,12]} d Q_{[5,6,7,8],[13,14,15,16]} d Q_{[5,6,7,8,10,11],[11,12,13,14,15,16]}\right) \\
& \left(-\mathrm{d} \mathrm{Q}_{[13,14,15,16],[8,9,11,12]} a_{1}+\mathrm{d} \mathrm{Q}_{[13,14,15,16],[8,9,10,11]} a_{3}\right) \\
& -\mathrm{dQ}_{[5,6,7,8],[9,10,11,12]}\left(\mathrm{d} \mathrm{Q}_{[13,14,15,16],[6,7,8,9]} c_{1} 6+c_{2} \mathrm{dQ}_{[5,6,7,8],[13,14,15,16]}\right) \\
& \left.\left(a_{2} a_{3}+a_{1} b_{5}\right)\right) A[11,11]
\end{aligned}
$$

where each of the $a_{i}$ S represents a six term quadratic polynomial in minors of $Q$. The $b_{i}$ s represent ten term quadratics in minors of $Q$. The $c_{i}$ s represent two term quadratics in minors of $Q$. 
The author has little doubt that equation 3.153 and its counterparts can be simplified significantly if enough time, energy, and computing power are devoted to the cause. When this last set of minors is solved linearly for eight more of the $A_{i, j} \mathrm{~s}$ in terms of the remaining $A_{i, j}$ s, we are left with a 32 parameter family of solutions for the modified $4 \times 4$ problem. Therefore, we have 32 parameter family data sets for each of the four $2 \times 2$ subsystems. The last step is to solve each of these subsystems as done in section 3.2.1. The solution for each of the $2 \times 2$ subsystems is a 16 parameter family of solutions in terms of the data. The process of solving all four subsystems introduces another $64=4 * 16$ parameters and yields the result promised at the beginning of section 3.2.3: a $96=4 * 16+32$ parameter family of solutions for the unknown transition probabilities for a $4 \times 4$ system.

\subsection{4 $n \times n$ problem where $n=2^{k}, k \in \mathbb{N}$}

In the previous section two recursive levels were required to solve the $4 \times 4$ problem. A sketch of the author's vision of the algorithm for the $8 \times 8$ problem follows. Later, the algorithm for a $n \times n$ problem is sketched.

The first step in tackling the $8 \times 8$ problem is to break up the $8 \times 8$ system into four $4 \times 4$ subsystems. See figure 3.1. Only 32 of the original $8^{2} * 16-32=992$ hidden states are considered in this modified system. The modified transition probabilities are the probabilities with which a photon travels from one of the pertinent states to another such that its travel path lies entirely inside one of the subsystems. These modified transition probabilities comprise the data for the $4 \times 4$ subsystems. Furthermore, the same process for solving the governing equations 2.8 that was used in sections 3.2.1 and 3.2.3.1 permits expression of the modified transition probabilities in terms of the entries of $A=P_{h o}^{-1} . P_{h o}$ is a $32 \times 32$ block diagonal matrix with four $8 \times 8$ blocks along its diagonal. Since $A$ has the same zero structure, we have a $4 * 8^{2}=256$ parameter solutions for the modified transition probabilities. There are many consistency conditions amongst the data for each of the $4 \times 4$ subsystems. These conditions should allow us to solve for all but $64 A_{i, j} \mathrm{~s}$ in terms of the remaining $A_{i, j}$ s. 
Notation: Le' $A, P_{h o}, P_{i o}, P_{h h}$, and $P_{i h}$ denote the modified transition matrices at the first level of this recursive algorithm. At the next level of the recursive process each of the four $4 \times 4$ subsystems will have its own data matrix, $Q i_{1} j_{1}$, where we refer to the subsystems as system $i_{1} j_{1}$, where $i_{1}, j_{1}=1,2$. The transition matrices for the modified $4 \times 4$ system $i_{1} j_{1}$ are referred to as $A i_{1} j_{1}, P i_{1} j_{1 h o}, P i_{1} j_{1_{i o}}, P i_{1} j_{1_{h h}}$, and $P i_{1} j_{1_{i h}}$. At the last recursive level of this recovery algorithm, each of the $4 \times 4$ modified systems will be broken into four $2 \times 2$ subsystems. The $\left(i_{2}, j_{2}\right)^{\text {th }} 2 \times 2$ subsystem of the $\left(i_{1}, j_{1}\right)^{\text {th }} 4 \times 4$ subsystem will be referred to as system $i_{1} j_{1_{i_{2}} j_{2}}$. The data matrices for these sub-subsystems will be referred to as $Q i_{1} j_{1_{i_{2} j_{2}}}$; the transition matrices as $P i_{1} j_{1_{i_{2} j_{2} h o}}, P i_{1} j_{1_{i_{2} j_{2} j_{o}}}, P i_{1} j_{1_{i_{2} j_{2} h h}}$, and $P i_{1} j_{i_{2} j_{2} \text { ih }}$.

Now that we have 64 parameter solutions for $Q 11, Q 12, Q 21$, and $Q 22$ we can implement the recovery algorithm for the $4 \times 4$ problem on each of the $4 \times 4$ subsystems as done in section 3.2.3. See figure 3.2. For subsystem $i_{1} j_{1}$ we recover $Q i_{1} j_{1_{i_{2} j_{2}}}$ for each combination $i_{2}, j_{2}=1,2$ in terms of $Q i_{1} j_{1}$ and half of the nonzero entries of $A i_{1} j_{1}$. Since each $A i_{1} j_{1}$ is a $16 \times 16$ block diagonal matrix with four $4 \times 4$ blocks along the diagonal, we introduce $1 / 2 * 4 * 4 * 4^{2}=128$ additional parameters to our solutions for the data submatrices for the $2 \times 2$ sub-subsystems. The resulting data matrices, $Q i_{1} j_{1_{i_{2} j_{2}}}$, should be functions of $64+128=192$ parameters. Now, we must simpy implement the $2 \times 2$ recovery algorithm on each of the $4^{2}=16$ sub-subsystems. We may solve for each set of transition matrices $P i_{1} j_{i_{2} j_{2} h_{o}}, P i_{1} j_{i_{2} j_{2} i_{o}}, P i_{1} j_{1_{i_{2} j_{2} h h}}$, and $P i_{1} j_{1_{i_{2} j_{2} i h}}$ in terms of $A i_{1} j_{1_{i_{2} j_{2}}}$, introducing another $256=4^{2} * 16$ parameters. The end result is a $256+128+64=448=8 * 8(8-1)$ parameter family of solutions for the transition probabilities in terms of the data matrix $Q$. Recall from section 3.1 that the rank of the forward map is at most $8 n(n+1)$. For the $8 \times 8$ problem we can at best find a $16 n^{2}-\left.8 n(n+1)\right|_{n=8}=448$ parameter family of solutions. 
In general, the recovery algorithm for a $n \times n$ system where $n=2^{k}, k \in \mathbb{N}$, requires $k-1$ recusive levels before the $2 \times 2$ "base case" is reached. Pseudocode for this algorithm is shown below:

solveasubsystem := proc(sysin)

break up sysin into four $m \times m$ subsystems

if $m=2$ then

solve each subsystem for its transition probabilities in terms

of its data and 16 parameters

else for each subsystem

1. solve for modified transition probabilities in terms of data and $A$

2. eliminate all but $8 m$ parameters using consistency conditions

3. call solveasubsystem with this subsystem as input

fi; 


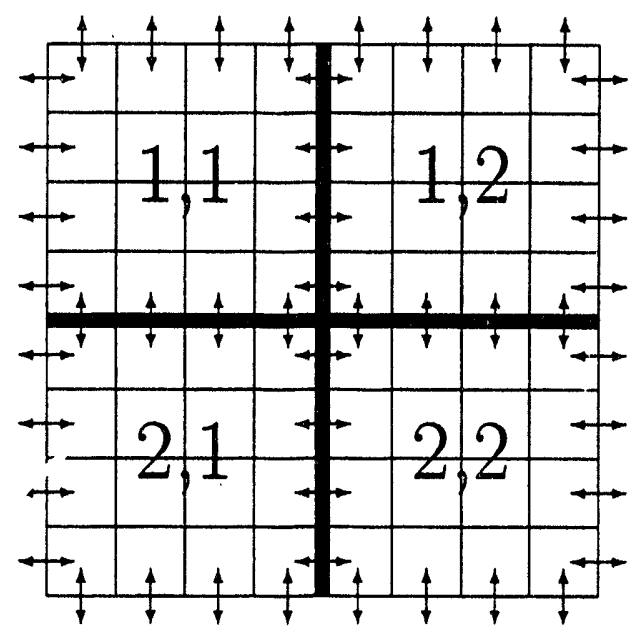

Figure 3.1: Decomposition of an $8 \times 8$ system into four $4 \times 4$ subsystems. The thick lines separate the subsystems. Only states which are considered when solving for the subsystems' data are denoted with arrows.
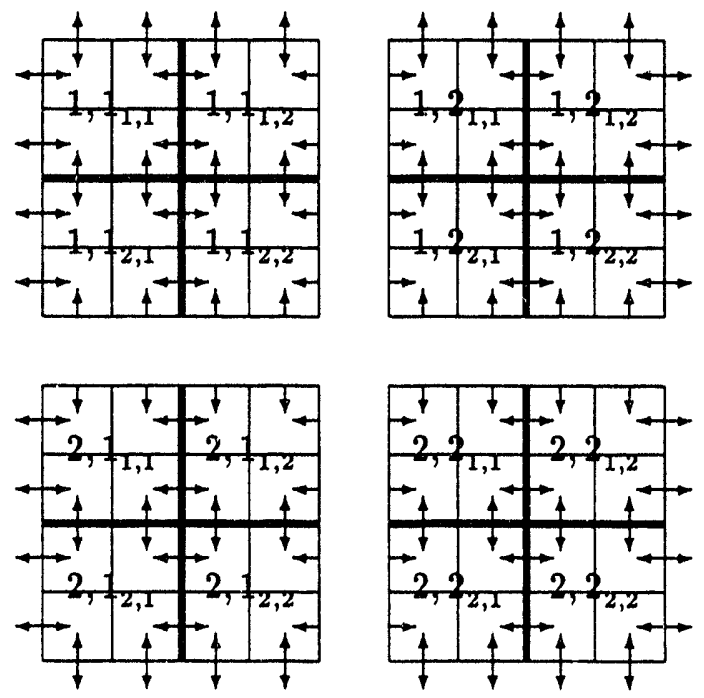

Figure 3.2: Decomposition of a $8 \times 8$ system into four $4 \times 4$ which are subsequently decomposed into $2 \times 2$ subsystems. The thick lines separate the subsystems. 


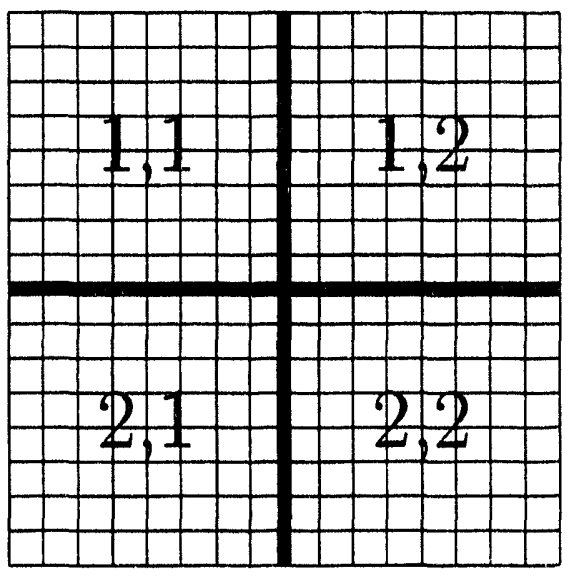

Figure 3.3: Decomposition of a $16 \times 16$ system into four $8 \times 8$ subsystems. The thick lines separate the subsystems.

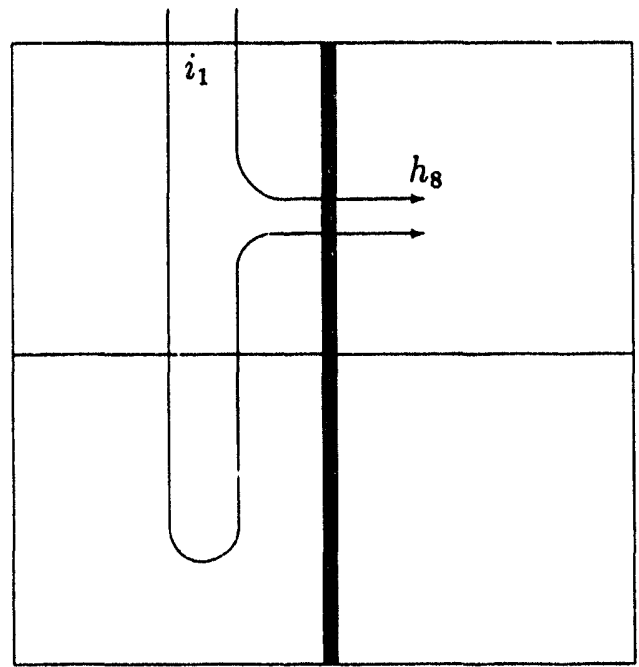

Figure 3.4: Two routes taken into account by $p_{1,8}$ 


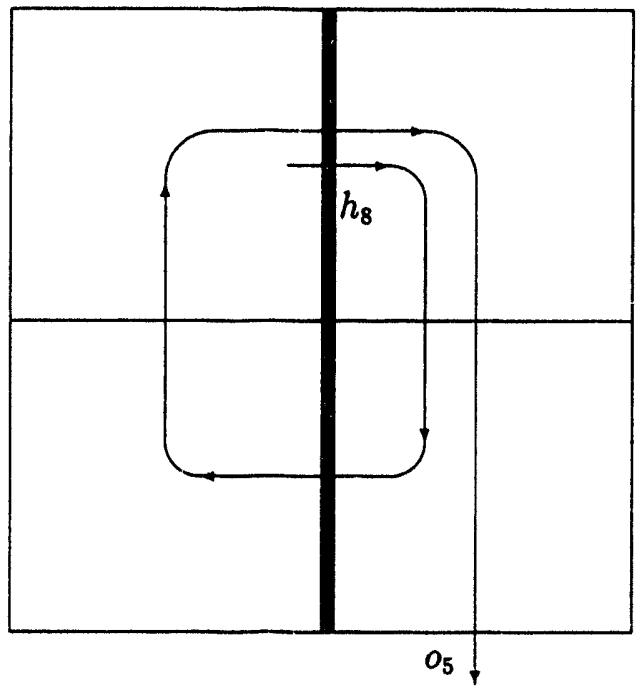

Figure 3.5: One of many paths taken into account by $s_{8,5}$

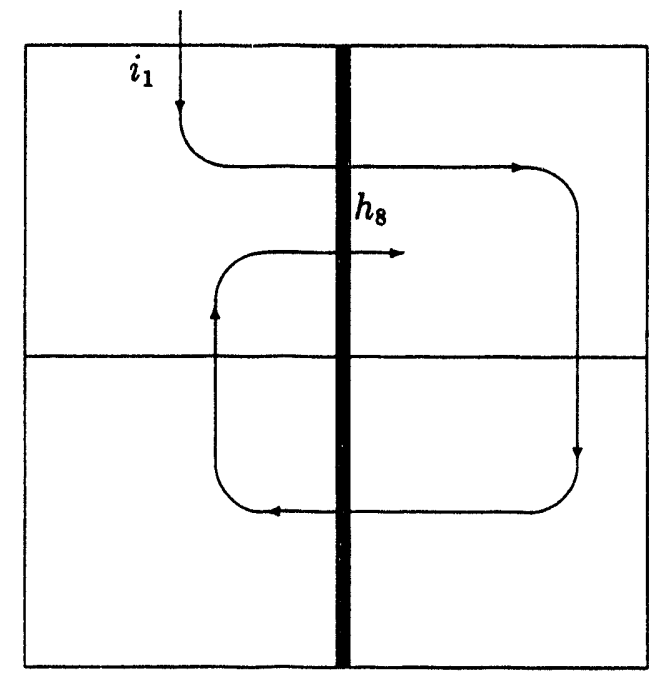

Figure 3.6: One route which $p_{1,8}$ does not take into account. 


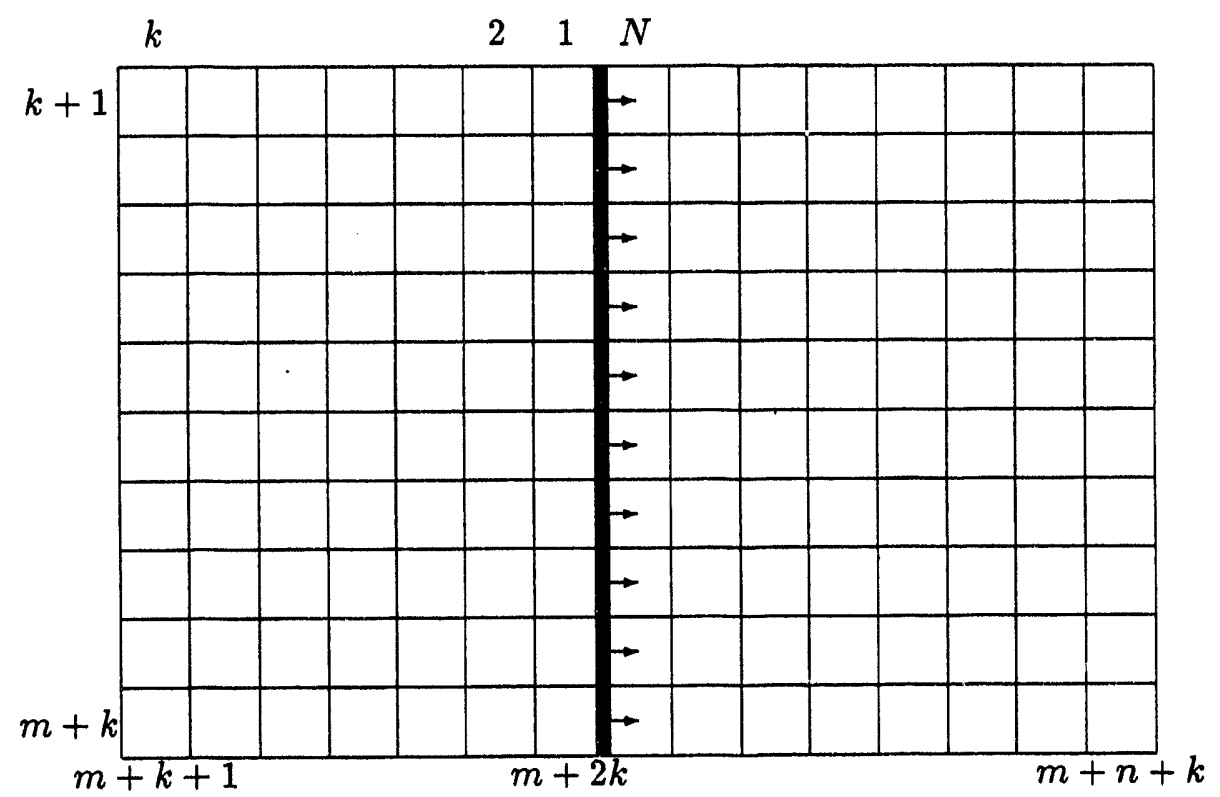

Figure 3.7: An example of a larger system. The thick veritical line separates the "left" states from "right" states. In order for a photon to travel from one of the sources left of the barrier to a detector right of the barrier, the photon must enter at least one of the states marked with arrows.

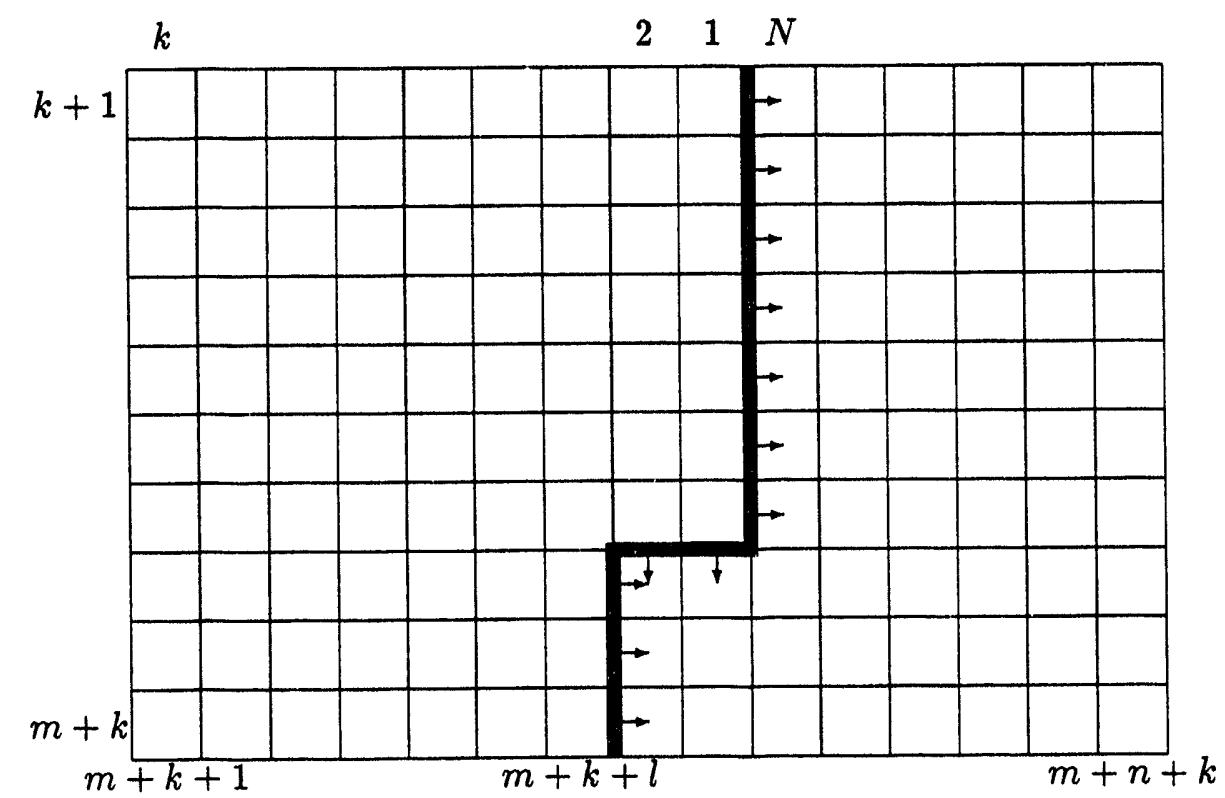

Figure 3.8: Example of a larger system with a weird boundary. In this particular example, $(k-l)=2$. 


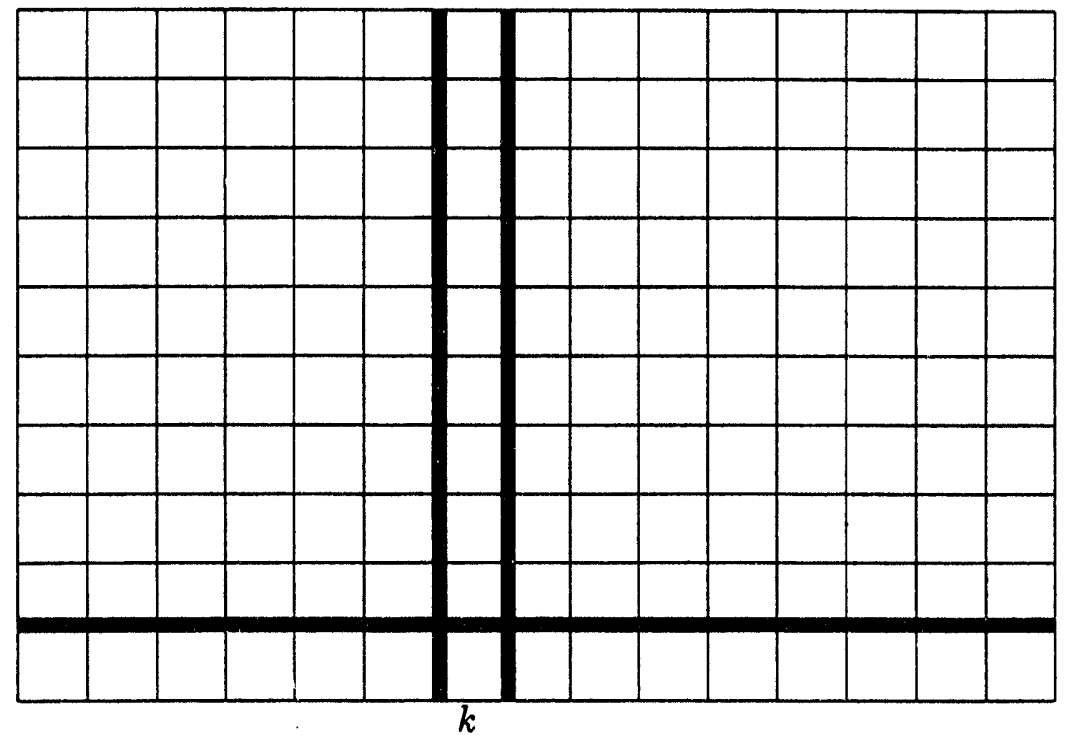

Figure 3.9: Source $k$ is surrounded by three barriers indicated by the thick lines.

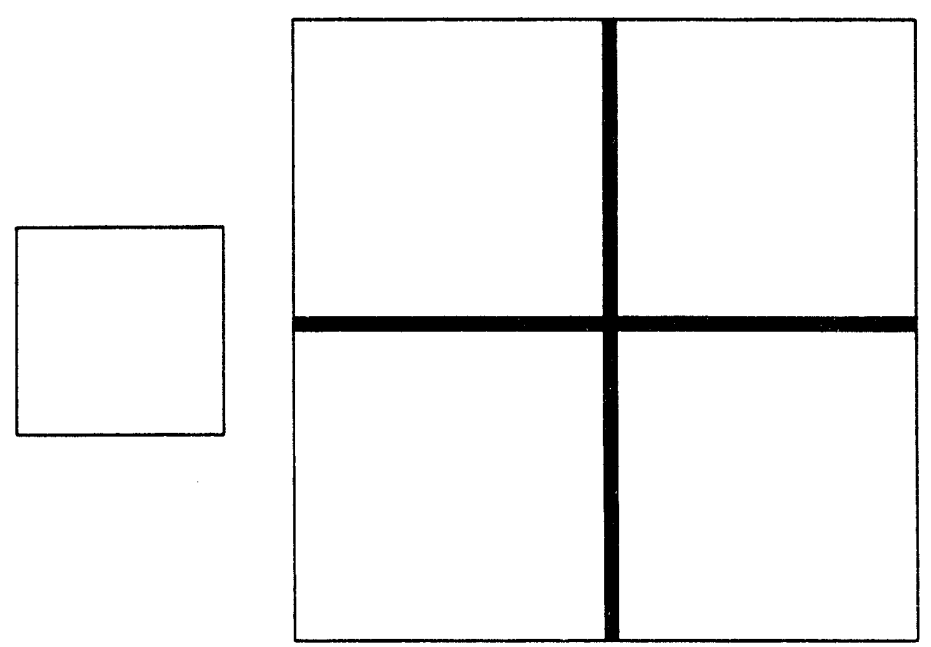

Figure 3.10: For the $1 \times 1$ case shown on the left, there are no boundaries. For the $2 \times 2$, case, however, there is one left-right and one top-bottom boundary. 


$$
\left[\begin{array}{llllllll}
i & i & & & & & x & x \\
i & i & & & & & x & x \\
x & x & i & i & & & & \\
x & x & i & i & & & & \\
& & x & x & i & i & & \\
& & & & i & i & & \\
& & x & x & i & & & \\
& & & & x & x & i & i \\
& & & & & & & \\
& & & & x & x & i & i
\end{array}\right]
$$

Figure 3.11: Data for the $2 \times 2$ problem which may be considered redundant due to $4 \times 4$ rank two submatrices are marked with an ' $x$ '. Data which are independent of all consistency conditions are marked with an ' $i$ '. The independent data correspond to nonzero entries of $P_{i o}$. Note that the choice of redundant data is not unique. 


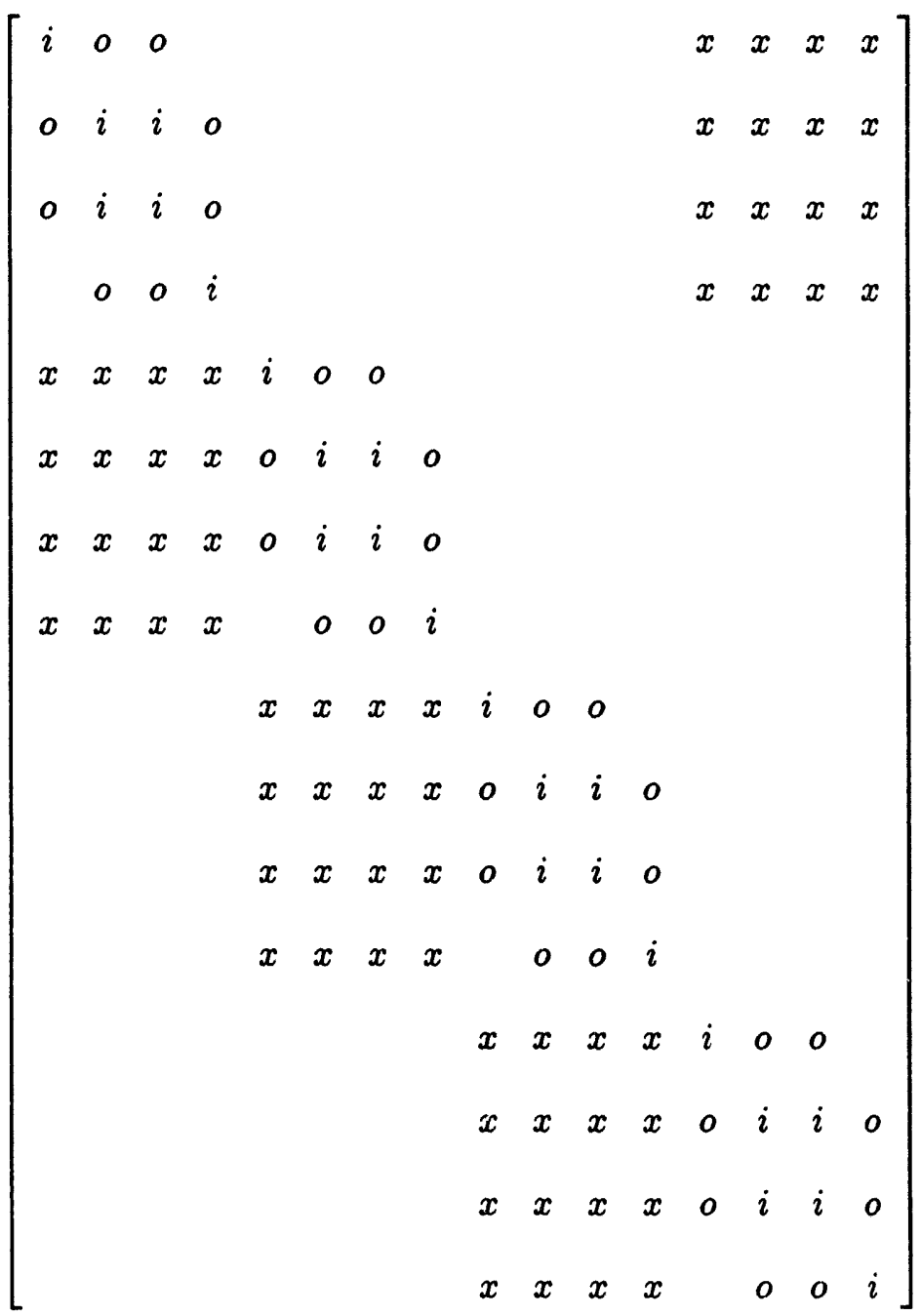

Figure 3.12: Data which may be considered redundant due to $8 \times 8$ rank four submatrices are marked with an ' $x$ '. Data which may be considered redundant due to $6 \times 10$ or $10 \times 6$ rank four submatrices are marked with an ' $o$ '. Data which are independent of all consistency conditions are labeled with an ' $i$ '. As before, the choice of redundant data is not unique. 


$$
\left[\begin{array}{cccccccc}
i & o_{1} & o_{1} & o_{1} & o_{1} & o_{1} & o_{1} & \\
o_{1} & i & o_{2} & o_{2} & o_{2} & o_{2} & & o_{1} \\
o_{1} & o_{2} & i & o_{3} & o_{3} & & o_{2} & o_{1} \\
o_{1} & o_{2} & o_{3} & i & i & o_{3} & o_{2} & o_{1} \\
o_{1} & o_{2} & o_{3} & i & i & o_{3} & o_{2} & o_{1} \\
o_{1} & o_{2} & & o_{3} & o_{3} & i & o_{2} & o_{1} \\
o_{1} & & o_{2} & o_{2} & o_{2} & o_{2} & i & o_{1} \\
& o_{1} & o_{1} & o_{1} & o_{1} & o_{1} & o_{1} & i
\end{array}\right]
$$

Figure 3.13: In any one of the $4 \times 4$ blocks on the diagonal of the data matrix for the $8 \times 8$ problem, data which may be considered redundant due to $18 \times 14$ or $14 \times 18$ rank eight submatrices are marked with an ' $o_{1}$ '. Data which may be considered redundant due to $20 \times 12$ or $12 \times 20$ rank eight submatrices are marked with an ' $o_{2}$ '. Data which may be considered redundant due to $22 \times 10$ or $10 \times 22$ rank eight submatrices are marked with an ' $O_{3}$ '. Data which are independent of all consistency conditions are labeled with ' $i$ '. Once again, this choice of redundant data is not unique. 


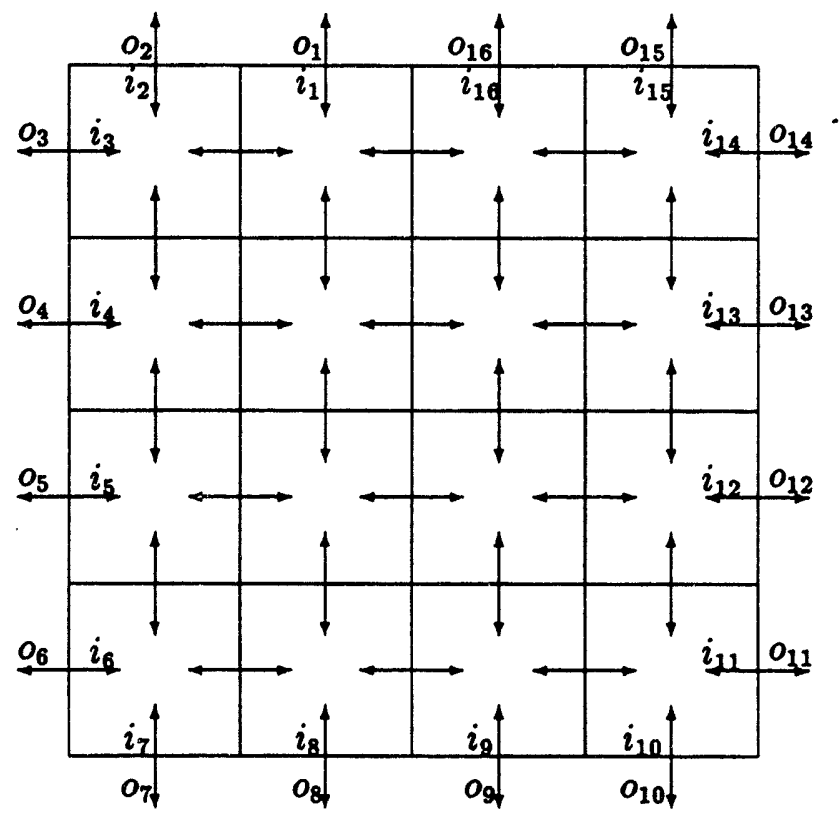

Figure 3.14: A $4 \times 4$ system. The incoming and outgoing states are labeled; all unlabeled states are hidden states. There are 16 incoming and 16 outgoing states, but 48 hidden states. 


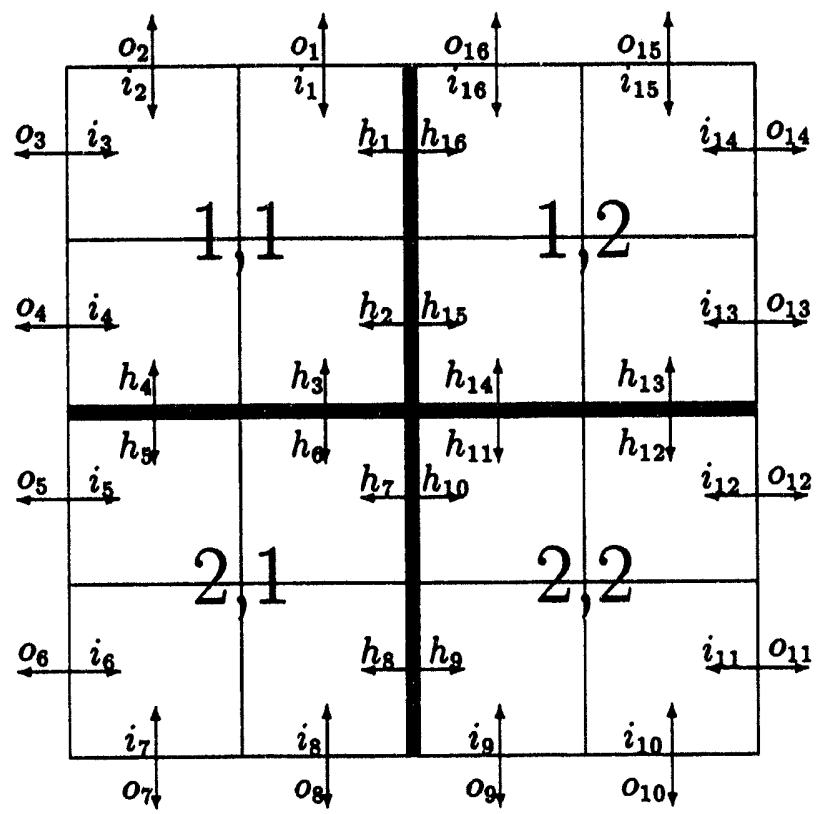

Figure 3.15: Decomposition of a $4 \times 4$ system into four $2 \times 2$ subsystems. The thick lines separate the subsystems. The "modified" $4 \times 4$ system disregards individual pixels. Only the subsystems are relevant at the first level of this recursive procedure. 


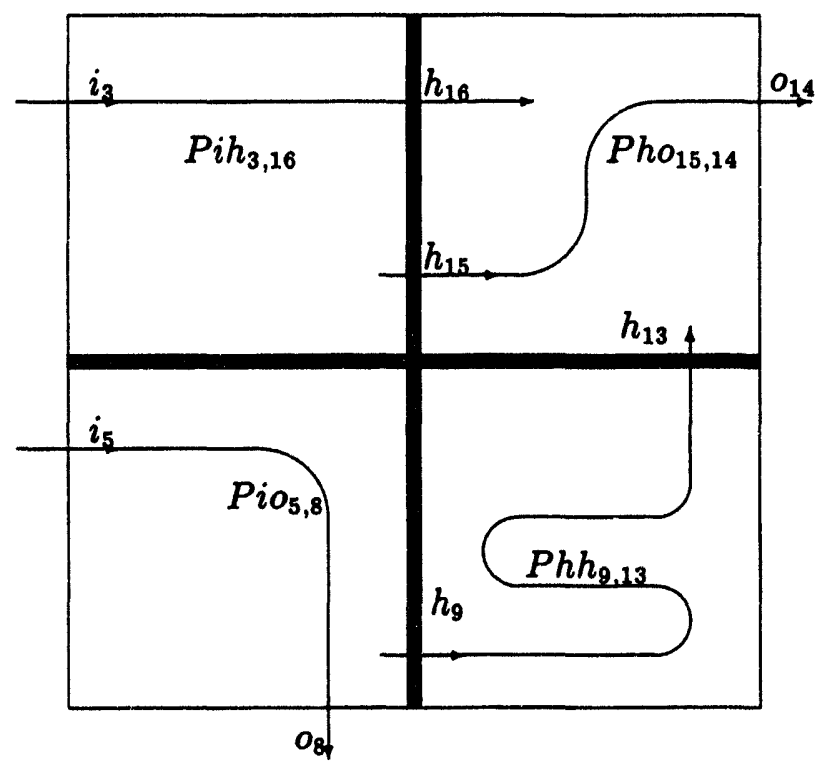

Figure 3.16: Examples of paths which are taken into account by transition probabilities for this modified system. 


\section{Chapter 4}

\section{Three Dimensional Problems}

\subsection{Introduction}

The setup is essentially the same in three dimensions as it was in two. An $n \times n \times n$ array of voxels in $\mathbb{R}^{3}$ encloses the object to be reconstructed. There are $6 n^{2}$ outer faces, each with a source and a detector. Preferred directions of travel are north, south, east, west, $u \mathrm{p}$, and down. The transitions matrices are larger than the matrices for an $n \times n$ problem and there are relatively more hidden states in three dimensicus. The governing matrix equation 2.8 is unchanged, however.

For a $n \times n \times n$ system there are $6 n^{2}$ incoming, $6 n^{2}$ outgoing, and $6 n^{3}-6 n^{2}=$ $6 n^{2}(6 n-1)$ hidden states. $P_{i o}$ is a $6 n^{2} \times 6 n^{2}$ matrix, while $P_{i h}$ is $6 n^{2} \times 6 n^{2}(n-1), P_{h h}$ is $6 n^{2}(n-1) \times 6 n^{2}(n-1)$, and $P_{h o}$ is $6 n^{2}(n-1) \times 6 n^{2} . Q$ is a $6 n^{2} \times 6 n^{2}$ matrix.

One of the differences between the two and three dimensional problems is the amount of data. In two dimensions there is precisely as much data as there are unknown transition probabilities. In three dimensions there are $36 n^{4}$ pieces of data but only $36 n^{3}$ unknowns. (There are six ways to enter a voxel and six ways to exit; each voxel has $6^{2}=36$ transition probabilities.) Since the rank of the forward map cannot be greater than the dimension of its domain, there must be consistency conditions upon the entries of $Q$. In fact, there are enough conditions to make the three dimensional forward map rank deficient. In [13] it is shown that the Jacobian for the $2 \times 2 \times 2$ problem is generically only of rank 240 . In the following sections we shall express the unknown transition probabilities in terms of a $36 * 2^{3}-240=48$ free parameters and the data. 


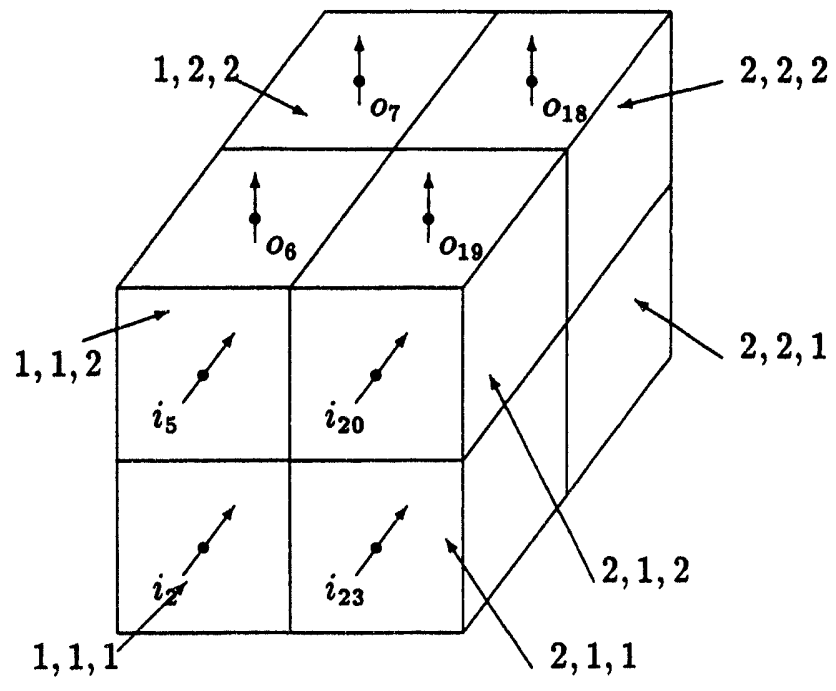

Figure 4.1: Eight voxels, seven of which are labelled above. Voxel 1,2,1 is hidden from view. Some incoming and outgoing states are labeled as well. A photon which travels north into voxel 112 via incoming state $i_{5}$ and then turns upward traveling out of voxel 112 via outgoing state $o_{6}$ does so with probability $n 112 u$.

Notation : The probability that the photon will travel east into pixel 1,1,1 and continue east into pixel $2,1,1$ is written as e111e. The probability that it will turn right and travel out of the system is written as $e 111 s$ and the probability with which it turns upwards and travel into pixel $1,1,2$ is written as $e 111 n$.

The transition probabilities satisfy 2.9 (as mentioned in chapter 2)

$$
\begin{aligned}
e i j k e+e i j k w+e i j k n+e i j k s+e i j k u+e i j k d & \leq 1 \\
u i j k e+u i j k w+u i j k n+u i j k s+u i j k u+u i j k d & \leq 1 \\
d i j k e+d i j k w+d i j k n+d i j k s+d i j k u+d i j k d & \leq 1 \\
w i j k e+w i j k w+w i j k n+w i j k s+w i j k u+w i j k d & \leq 1 \\
n i j k e+n i j k w+n i j k n+n i j k s+n i j k u+n i j k d & \leq 1 \\
s i j k e+s i j k w+s i j k n+s i j k s+s i j k u+s i j k d & \leq 1
\end{aligned}
$$

where $i, j, k=1,2$ 

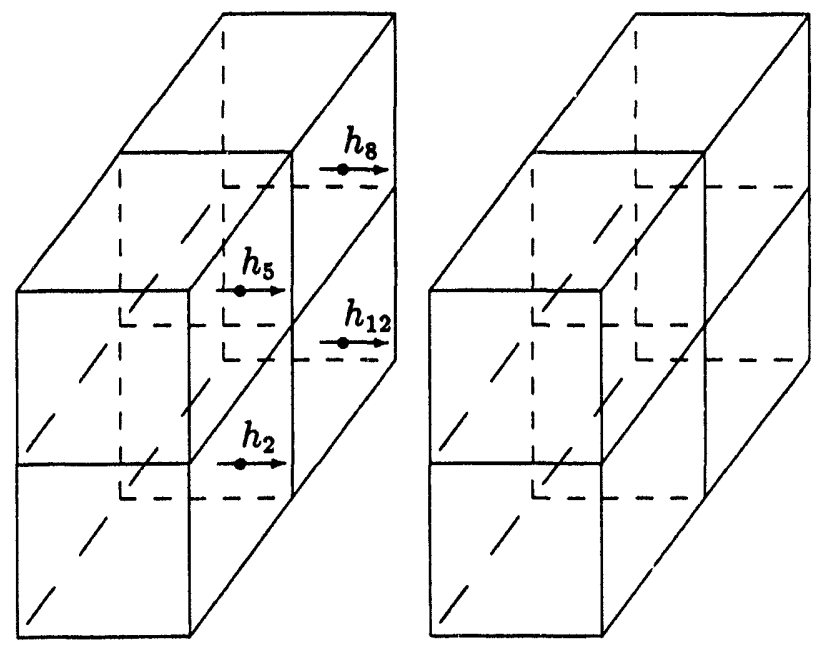

Figure 4.2: A $2 \times 2 \times 2$ system is split apart so that we can see a few hidden states representing travel from the "leftmost" voxels, 111, 121, 112, and 122, to the "rightmost" voxels, 211 , 221,212 , and 222 .

Furthermore, this system satisfies the range conditions (also mentioned in chapter 2)

$$
0 \leq \sum_{\lambda=1}^{24} Q_{i, \lambda} \leq 1 \quad i=1,2, \ldots, 6 n^{2}
$$

The method for finding a 48 parameter family of solutions to the inverse problem starts off just like the two dimensional method. The matrix equations $2.8,3.18$, and 3.18 , are the same; only the matrices are different. The matrices for the $2 \times 2 \times 2$ problem also have special block structures. $P_{i o}$ and $P_{h o}$ are $24 \times 24$ block diagonal matrices with eight $3 \times 3$ blocks. $P_{i h}$ and $P_{h h}$ are $24 \times 24$ matrices with nonzero entries on their off diagonals. As before, $A$ and $X$ have the same diagonal block structure as $P_{h o} ; W$ and $Y$ have the same off diagonal block structure as $P_{h h}$ and $P_{i h}$. For the $2 \times 2 \times 2$ problem, each column in 3.18 is a system of 24 linear equations in the 12 variables which appear in the corresponding columns of $A-W$ and $X-Y$. Just as in two dimensions, the columns of $A-W$ and $X-Y$ come in pairs. The roles of the $A_{i, j} s$ and $W_{i, j} s$ are reversed in the first and last columns of $A-W$ as are the roles of the $X_{i, j} s$ and $Y_{i, j} s$ in the first and last columns of $X-Y$. Hence, we must solve the "same" matrix equation for the first and last columns of 3.18. See 
table 4.1. We shall consider column three of 3.18. The third columns of $A-W$ and $X-Y$ are shown below:

$$
\left[\begin{array}{c}
A_{1,3} \\
A_{2,3} \\
A_{3,3} \\
-W_{4,3} \\
-W_{5,3} \\
-W_{6,3} \\
\theta
\end{array}\right] \quad\left[\begin{array}{c}
X_{1,3} \\
X_{2,3} \\
X_{3,3} \\
-Y_{4,3} \\
-Y_{5,3} \\
-Y_{6,3} \\
\theta
\end{array}\right]
$$

respectively, where $\theta$ is a column vector of eighteen zeros. The 24 equations in column three can be written as a homogeneous matrix equation: 
$\left[\begin{array}{llllllllllll}Q_{1,1} & Q_{1,2} & Q_{1,3} & Q_{1,4} & Q_{1,5} & Q_{1,6} & -1 & 0 & 0 & 0 & 0 & 0\end{array}\right]$ $\begin{array}{llllllllllll}Q_{2,1} & Q_{2,2} & Q_{2,3} & Q_{2,4} & Q_{2,5} & Q_{2,6} & 0 & -1 & 0 & 0 & 0 & 0\end{array}$ $\begin{array}{llllllllllll}Q_{3,1} & Q_{3,2} & Q_{3,3} & Q_{3,4} & Q_{3,5} & Q_{3,6} & 0 & 0 & -1 & 0 & 0 & 0\end{array}$ $\begin{array}{llllllllllll}Q_{4,1} & Q_{4,2} & Q_{4,3} & Q_{4,4} & Q_{4,5} & Q_{4,6} & 0 & 0 & 0 & -1 & 0 & 0\end{array}$ $\begin{array}{llllllllllll}Q_{5,1} & Q_{5,2} & Q_{5,3} & Q_{5,4} & Q_{5,5} & Q_{5,6} & 0 & 0 & 0 & 0 & -1 & 0\end{array}$ $\begin{array}{llllllllllll}Q_{6,1} & Q_{6,2} & Q_{6,3} & Q_{6,4} & Q_{6,5} & Q_{6,6} & 0 & 0 & 0 & 0 & 0 & -1\end{array}$ $\begin{array}{llllllllllll}Q_{7,1} & Q_{7,2} & Q_{7,3} & Q_{7,4} & Q_{7,5} & Q_{7,6} & 0 & 0 & 0 & 0 & 0 & 0\end{array}$ $\begin{array}{llllllllllll}Q_{8,1} & Q_{8,2} & Q_{8,3} & Q_{8,4} & Q_{8,5} & Q_{8,6} & 0 & 0 & 0 & 0 & 0 & 0\end{array}$ $\begin{array}{llllllllllll}Q_{9,1} & Q_{9,2} & Q_{9,3} & Q_{9,4} & Q_{9,5} & Q_{9,6} & 0 & 0 & 0 & 0 & 0 & 0\end{array}$ $\begin{array}{llllllllllll}Q_{10,1} & Q_{10,2} & Q_{10,3} & Q_{10,4} & Q_{10,5} & Q_{10,6} & 0 & 0 & 0 & 0 & 0 & 0\end{array}$ $\begin{array}{llllllllllll}Q_{11,1} & Q_{11,2} & Q_{11,3} & Q_{11,4} & Q_{11,5} & Q_{11,6} & 0 & 0 & 0 & 0 & 0 & 0\end{array}$ $\begin{array}{llllllllllll}Q_{12,1} & Q_{12,2} & Q_{12,3} & Q_{12,4} & Q_{12,5} & Q_{12,6} & 0 & 0 & 0 & 0 & 0 & 0\end{array}$ $\begin{array}{llllllllllll}Q_{13,1} & Q_{13,2} & Q_{13,3} & Q_{13,4} & Q_{13,5} & Q_{13,6} & 0 & 0 & 0 & 0 & 0 & 0\end{array}$ $\begin{array}{llllllllllll}Q_{14,1} & Q_{14,2} & Q_{14,3} & Q_{14,4} & Q_{14,5} & Q_{14,6} & 0 & 0 & 0 & 0 & 0 & 0\end{array}$ $\begin{array}{llllllllllll}Q_{15,1} & Q_{15,2} & Q_{15,3} & Q_{15,4} & Q_{15,5} & Q_{15,6} & 0 & 0 & 0 & 0 & 0 & 0\end{array}$ $\begin{array}{llllllllllll}Q_{16,1} & Q_{16,2} & Q_{16,3} & Q_{16,4} & Q_{16,5} & Q_{16,6} & 0 & 0 & 0 & 0 & 0 & 0\end{array}$ $\begin{array}{llllllllllll}Q_{17,1} & Q_{17,2} & Q_{17,3} & Q_{17,4} & Q_{17,5} & Q_{17,6} & 0 & 0 & 0 & 0 & 0 & 0\end{array}$ $\begin{array}{llllllllllll}Q_{18,1} & Q_{18,2} & Q_{18,3} & Q_{18,4} & Q_{18,5} & Q_{18,6} & 0 & 0 & 0 & 0 & 0 & 0\end{array}$ $\begin{array}{llllllllllll}Q_{19,1} & Q_{19,2} & Q_{19,3} & Q_{19,4} & Q_{19,5} & Q_{19,6} & 0 & 0 & 0 & 0 & 0 & 0\end{array}$ $\begin{array}{llllllllllll}Q_{20,1} & Q_{20,2} & Q_{20,3} & Q_{20,4} & Q_{20,5} & Q_{20,8} & 0 & 0 & 0 & 0 & 0 & 0\end{array}$ $\begin{array}{llllllllllll}Q_{21,1} & Q_{21,2} & Q_{21,3} & Q_{21,4} & Q_{21,5} & Q_{21,6} & 0 & 0 & 0 & 0 & 0 & 0\end{array}$ $\begin{array}{llllllllllll}Q_{22,1} & Q_{22,2} & Q_{22,3} & Q_{22,4} & Q_{22,5} & Q_{22,6} & 0 & 0 & 0 & 0 & 0 & 0\end{array}$ $\begin{array}{llllllllllll}Q_{23,1} & Q_{23,2} & Q_{23,3} & Q_{23,4} & Q_{23,5} & Q_{23,6} & 0 & 0 & 0 & 0 & 0 & 0\end{array}$ $\left[\begin{array}{llllllllllll}Q_{24,1} & Q_{24,2} & Q_{24,3} & Q_{24,4} & Q_{24,5} & Q_{24,6} & 0 & 0 & 0 & 0 & 0 & 0\end{array}\right]$ $\left.\begin{array}{c}A_{1,3} \\ A_{2,3} \\ A_{3,3} \\ W_{4,3} \\ W_{5,3} \\ W_{6,3} \\ X_{1,3} \\ X_{2,3} \\ X_{3,3} \\ Y_{4,3} \\ Y_{5,3} \\ Y_{6,3}\end{array}\right]$ 
We have twelve sets of homogeneous linear equations like 4.4 corresponding to twelve $24 \times 12$ matrices which satisfy the homogeneous equation $C x=\theta$. Since the trivial solution would not be interesting enough to write about one may safely assume that there must be other solutions. This is indeed the case since the lower left $18 \times 6$ submatrix found in equation 4.4, represents travel into voxels 111 and 112 from the the other six voxels. As shown in [13] this submatrix is of rank four or less. Since the first six equations in 4.4 are independent, we may solve 4.4 for at most $6+4=10$ of the twelve unknowns in terms of the other two.

\subsection{Solving the Equations}

Since the $W_{i, j} s, X_{i, j} s$, and $Y_{i, j} s$ are already functions of $A_{i, j} s$, it seems natural to solve for them in terms of the $A_{i, j} s$. Following this procedure for all 24 columns reduces the number of unknowns from 288 to 72 . The analogous procedure in two dimensions exhausts the supply of independent equations. In three dimensions, however, we have enough information to solve for one third of the $A_{i, j} \mathrm{~s}$ in terms of the remaining $A_{i, j} \mathrm{~s}$.

To solve 4.4 for the $W_{i, j} s, X_{i, j} s, Y_{i, j} s$, and $\operatorname{diag}(A)$ in terms of the rest of the $A_{i, j} s$, one need only solve:

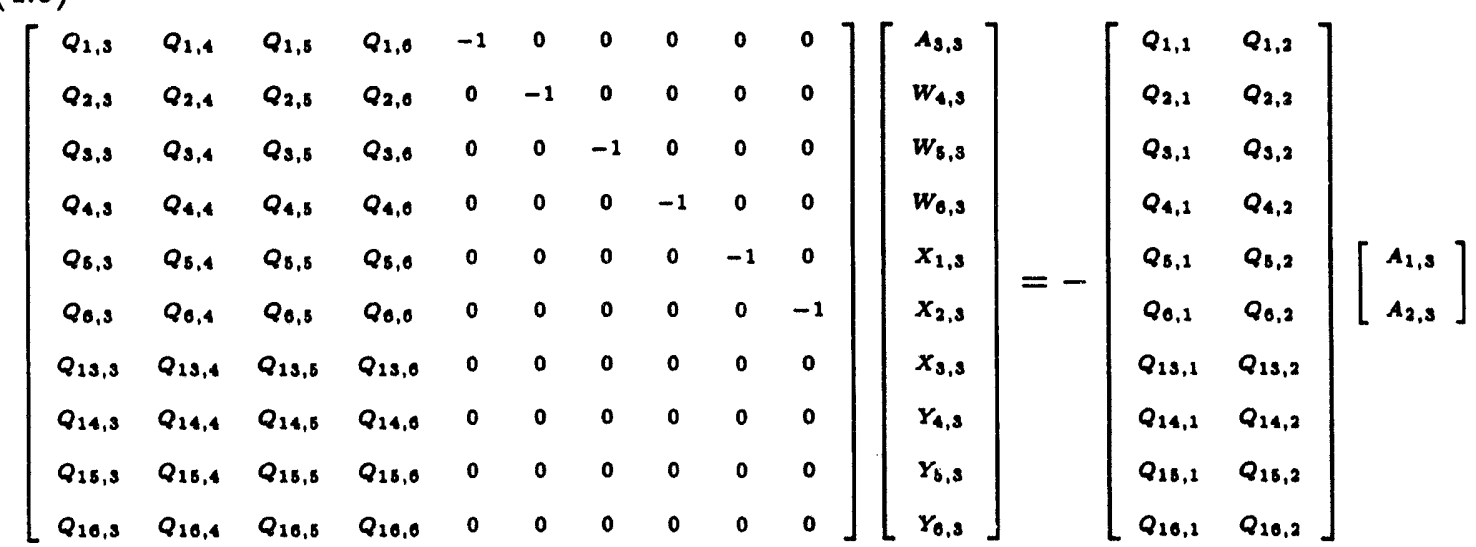

Notation : Denote the determinant of the submatrix of $Q$ taken from rows $\left[r_{1}, r_{2}, \ldots, r_{n}\right]$ and columns $\left[c_{1}, c_{2}, \ldots, c_{n}\right]$ as $d Q_{\left[r_{1}, r_{2}, \ldots, r_{n}\right],\left\{c_{1}, c_{2}, \ldots, c_{n}\right]}$ 


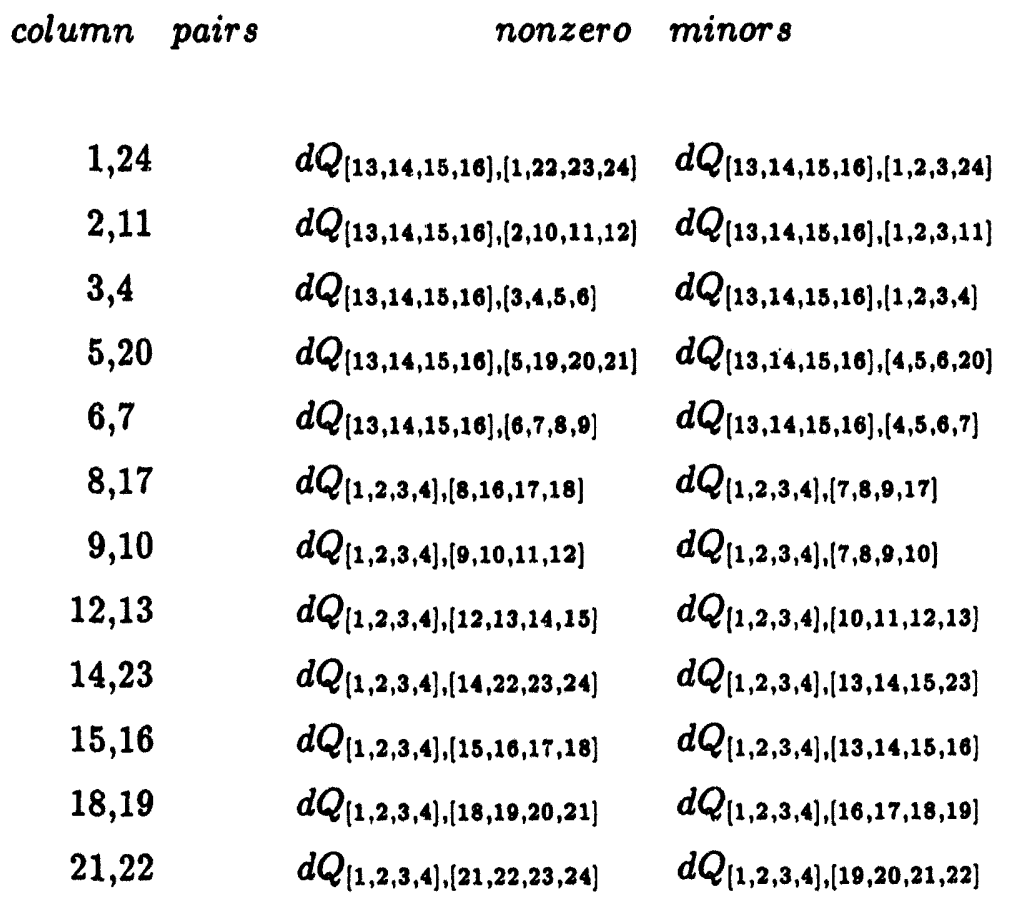

Table 4.1: The columns of 3.18 come in pairs. Each pair is shown in the left hand column. In order to solve a "column of equations" in 3.18 we require that a minor of $Q$ is nonzero. These minors are displayed to the right of their corresponding column numbers.

The determinant of the lefthand matrix in 4.5 is $d Q_{[13,14,15,16],(3,4,5,6]}$. Equation 4.5 has a unique solution if and only if $d Q_{[13,14,15,16],[3,4,5,6]} \neq 0$. The same sort of requirement holds for each of the other columns of 3.18. Although there are only twelve different (and underdetermined!) matrix equations in terms of the unknowns, we must solve 24 different linear systems of equations in order to solve for the $W_{i, j} s, X_{i, j} s, Y_{i, j} s$ and $\operatorname{diag}(A)$ in terms of the rest of the $A_{i, j} s$. Table 4.1 shows which columns correspond to the same matrix equation and minors of the data we require to be nonzero.

If the data satisfy these requirenents then we can solve the 240 independent equations in 288 variables linearly for the nonzero entries in $W, X, Y$, and $\operatorname{diag}(A)$ in terms of the 48 other variables in $A=P_{h o}^{-1}$. (Note that this choice of equations is not unique.) 
Because the solutions for the transition probabilities in terms of all 72 of the $A_{i, j} \mathrm{~s}$ are much simpler than their solutions in terms of only the off diagonal elements of $A$, we first solve in terms of all of the entries of $A$. Sample solutions from each of the four one step transition submatrices shown below. Since $P_{h o}=A^{-1}$, the solutions for entries of $P_{h o}$ are especially simple:

$$
u 122 w=\frac{d A_{[7,8],[7,8]}}{d A_{[7,8,9],[7,8,9]}}
$$

Solutions for variables from a transition submatrix are all of the same form. For example, all of the transition probabilities in $P_{h o}$ are equal to a $2 \times 2$ minor of $A$ divided by a $3 \times 3$ minor of $A$. One of $P_{h h}$ 's nonzero entries may be written as

$$
\begin{gathered}
s 112 n=\left(A_{8,7} d A_{[4,5],[4,5]} \mathrm{dQ}\right. \\
A_{9,7} d A_{[4,5],\{4,5]} \mathrm{d} Q_{[13,14,15],[4,5,9]}+A_{8,7} d A_{[5,6],[4,5]} \mathrm{dQ}_{[13,14,15],[5,6,8]}+ \\
A_{9,7} d A_{[5,6],[4,5]} \mathrm{dQ}_{[13,14,15],[5,6,9]}+A_{7,7} d A_{[4,6],[4,5]} \mathrm{dQ}_{[13,14,15],[4,6,7]}+ \\
A_{7,7} d A_{[5,6],[4,5]} \mathrm{dQ}_{[13,14,15],[5,6,7]}+A_{8,7} d A_{[4,6],[4,5]} \mathrm{dQ}_{[13,14,15],[4,6,8]}+ \\
\left.A_{7,7} d A_{[4,5],[4,5]} \mathrm{dQ}_{[13,14,15],[4,5,7]}\right) /\left(d A_{[4,5,6],[4,5,6]} \mathrm{dQ}_{[13,14,15],[4,5,6]}\right)
\end{gathered}
$$

The solutions for entries of $P_{h o}$ and $P_{h h}$ were quite simple (for MAPLE) to compute. The polutions for transition probabilities in $P_{i o}$ and $P_{i h}$ appeared to be extremely messy at first. By grouping terms in the solutions for entries of $P_{i o}$ carefully it is possible to simplify them using matrix expansions of the forms

$$
\begin{aligned}
-d A_{[2,3],[1,3]} A_{3,2}+d A_{[2,3],[2,3]} A_{3,1}+d A_{[2,3],[1,2]} A_{3,3} & =0 \\
d A_{[1,2],[1,2]} A_{3,3}+d A_{[1,2],[2,3]} A_{3,1}-d A_{[1,2],[1,3]} A_{3,2} & =d A_{[1,2,3],[1,2,3]}
\end{aligned}
$$

The resulting solutions are quite simple:

$$
\begin{array}{r}
d 112 u=-d A_{[4,5],[5,6]}\left(\mathrm{dQ}_{[6,13,14,15],[1,2,3,4]} A_{4,4}+\mathrm{dQ}_{[6,13,14,15],[1,2,3,6]} A_{6,4}+\right. \\
\left.\mathrm{dQ}_{[6,13,14,15],[1,2,3,5]} A_{5,4}\right) / \mathrm{dQ}_{[13,14,15],[1,2,3]} d A_{[4,5,6],[4,5,6]}- \\
d A_{[4,5],[4,6]}\left(\mathrm{dQ}_{[6,13,14,15],[6,19,20,21]} A_{6,5}+\mathrm{dQ}_{[6,13,14,15],[4,19,20,21]} A_{4,5}+\right. \\
\left.\mathrm{dQ}_{[6,13,14,15],[5,19,20,21]} A_{5,5}\right) / \mathrm{dQ}_{[13,14,15],[19,20,21]} d A_{[4,5,6],[4,5,6]}+ \\
d A_{[4,5],[4,5]}\left(\mathrm{dQ}_{[6,13,14,15],[6,7,8,9]} A_{6,6}+\mathrm{d} \mathrm{Q}_{[6,13,14,15],[5,7,8,9]} A_{5,6}+\right. \\
\mathrm{dQ} \\
{[6,13,14,15],[4,7,8,9]} \\
\left.A_{4,6}\right) / \mathrm{dQ}_{[13,14,15],[7,8,9]} d A_{[4,5,6],[4,5,6]}
\end{array}
$$


The identities giving the Graßmann-Plücker embedding can be used to simplify the solutions for entries of $P_{i n}$ considerably. The method for simplifying these solutions is exactly the same as that used in $[7,6]$ and 3.2.1.3. One of the simplified solutions for a transition probability in $P_{i h}$ is shown below:

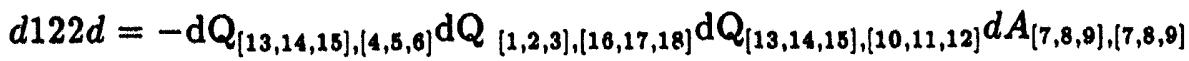

$$
\begin{aligned}
& \left(\mathrm{dQ}_{[7,13,14,15],[7,8,9,12]} A_{12,10}+\mathrm{dQ}_{[7,13,14,15],[7,8,9,10]} A_{10,10}+\mathrm{dQ}_{[7,13,14,15],(7,8,9,11]} A_{11,10}\right)+ \\
& \mathrm{dQ}_{[13,14,15],[4,5,8]} \mathrm{dQ} \mathrm{Q}_{[1,2,3],[16,17,18]}\left(A _ { 1 0 , 1 0 } \left(d A_{[7,8),[7,8]} \mathrm{dQ} \mathrm{Q}_{[13,14,15],[7,8,10]}+\right.\right. \\
& \left.d A_{[7,9],(7,8]} \mathrm{dQ}_{[13,14,15],(7,9,10]}+d A_{[8,9],[7,8]} \mathrm{d} \mathrm{Q}_{[13,14,15],(8,9,10]}\right)+ \\
& A_{12,10}\left(d A_{[7,9],[7,8]} \mathrm{dQ}_{[13,14,18],[7,9,12]^{+}}\right. \\
& \left.d A_{[8,9],[7,8]} \mathrm{dQ}_{[13,14,15],[8,9,12]}+d A_{[7,8],[7,8]} \mathrm{d} \mathrm{Q}_{[13,14,15],(7,8,12]}\right)+ \\
& A_{11,10}\left(d A_{[7,8],[7,8]} \mathrm{dQ}_{[13,14,15],[7,8,11]}+d A_{[8,9],[7,8]} \mathrm{dQ}_{[13,14,15],[8,9,11]}+\right. \\
& \left.\left.d A_{[7,9],[7,8]} \mathrm{dQ}_{[13,14,15],[7,9,11]}\right)\right)\left(\mathrm{dQ}_{[7,13,14,15],(7,10,11,12]} A_{7,9}+\right. \\
& \left.A_{8,9} \mathrm{dQ}_{[7,13,14,15],[8,10,11,12]}+A_{9,9} \mathrm{dQ}_{[7,13,14,15],[9,10,11,12]}\right)- \\
& \mathrm{dQ}_{[1,2,3],[16,17,18]} \mathrm{dQ}_{[13,14,15],[10,11,12]}\left(A _ { 1 2 , 1 0 } \left(d A_{[7,9],[8,9]} \mathrm{dQ}_{[13,14,15],\{7,9,12]}+\right.\right. \\
& \left.d A_{[7,8],[8,9]} \mathrm{d} \mathrm{Q}_{[13,14,15],[7,8,12]}+d A_{[8,9],[8,9]} \mathrm{dQ}_{[13,14,15],[8,9,12]}\right)+ \\
& A_{10,10}\left(d A_{[7,8],[8,9]} \mathrm{d} Q_{[13,14,15],[7,8,10]}+d A_{[8,9],(8,9]} \mathrm{dQ}_{[13,14,15],[8,9,10]}+\right. \\
& \left.d A_{[7,9],(8,9]} \mathrm{d} Q_{[13,14,15],(7,9,10]}\right)+ \\
& A_{11,10}\left(d A_{[8,9],[8,9]} \mathrm{dQ}_{[13,14,15],[8,9,11]}+d A_{[7,9],[8,9]} \mathrm{dQ}_{[13,14,15],[7,9,11]}+\right. \\
& \left.\left.d A_{[7,8],[8,9]} \mathrm{dQ} Q_{[13,14,15],[7,8,11]}\right)\right)\left(A_{9,7} \mathrm{dQ}_{[7,13,14,15],[4,5,6,9]}+\right. \\
& \left.A_{7,7} \mathrm{dQ}_{[7,13,14,15],[4,5,6,7]}+\mathrm{dQ}_{[7,13,14,15],(4,5,6,8]} A_{8,7}\right)+ \\
& \mathrm{dQ}_{[13,14,15],[4,5,6]} \mathrm{dQ}_{[13,14,15],\{10,11,12]}\left(A _ { 1 0 , 1 0 } \left(d A_{[8,9],[7,9]} \mathrm{dQ}_{[13,14,15],[8,9,10]}+\right.\right. \\
& \left.d A_{[7,9],[7,9]} \mathrm{d} \mathrm{Q}_{[13,14,15],(7,9,10]}+d A_{[7,8),[7,9]} \mathrm{dQ}_{[13,14,15],(7,8,10]}\right)+ \\
& A_{11,10}\left(d A_{[7,8],[7,9]} \mathrm{dQ}_{[13,14,15],[7,8,11]}+d A_{[7,9],[7,9]} \mathrm{dQ}_{[13,14,15],[7,9,11]}+\right. \\
& \left.d A_{[8,9],[7,9]} \mathrm{d} \mathrm{Q}_{[13,14,15],[8,9,11]}\right)+A_{12,10}\left(d A_{[7,8],[7,9]} \mathrm{d} \mathrm{Q}_{[13,14,15],[7,8,12]}+\right. \\
& \left.\left.d A_{[8,9],[7,9]} \mathrm{dQ}_{[13,14,15],[8,9,12]}+d A_{[7,9],[7,9]} \mathrm{dQ}_{[13,14,15],[7,9,12]}\right)\right) \\
& \left(A_{8,8} \mathrm{dQ}_{[1,2,3,7],[8,16,17,18]}+A_{7,8} \mathrm{dQ}_{[1,2,3,7],[7,16,17,18]}+\mathrm{dQ}_{[1,2,3,7],\{9,16,17,18]} A_{9,8}\right) / \\
& \left(d A_{[7,8,9],[7,8,9]} \mathrm{dQ}_{[13,14,15],\{7,8,9]} \mathrm{dQ}_{[13,14,15],[10,11,12]} \mathrm{dQ}_{[13,14,15],(4,5,6]} \mathrm{dQ}_{[1,2,3],[16,17,18]}\right)
\end{aligned}
$$


These solutions, $(4.10,4.10,4.6$, and 4.8$)$, are analogous to the sixteen parameter solution to the $2 \times 2$ problem where all of the transitions probabilities can be expressed in terms of the $A_{i, j}$ s. In this $2 \times 2 \times 2$ problem, however, we can solve for $\operatorname{diag}(A)$ in terms of the remaining $A_{i, j}$ s. One of the solutions for a diagonal entry of $A$ is shown below:

$$
A_{20,20}=-\frac{\mathrm{dQ}_{[13,14,13,16],[4,5,6,21]} A_{21,20}+\mathrm{dQ}_{[13,14,15,16],[4,5,6,19]} A_{19,20}}{\mathrm{dQ}_{[13,14,15,16],[4,5,6,20]}}
$$

Half of the solutions for $P_{h h}$ factor when the solutions for $\operatorname{diag}(A)$ are substituted into their numerators. Here is one example:

$$
\begin{aligned}
n 122 d= & \left(-A_{7,8} \mathrm{~d} Q_{[13,14,15,16],[8,10,11,12]} \mathrm{dQ}_{[1,2,3,4],[7,16,17,18]}-\right. \\
& A_{9,8} \mathrm{dQ}_{[13,14,15,16],[8,10,11,12]} \mathrm{dQ}_{[1,2,3,4],[9,16,17,18]}+ \\
& A_{9,8} \mathrm{dQ}_{[1,2,3,4],[8,16,17,18]} \mathrm{dQ}_{[13,14,15,16],[9,10,11,12]}+ \\
& \left.A_{7,8} \mathrm{dQ}_{[1,2,3,4],[8,16,17,18]} \mathrm{dQ}_{[13,14,15,16],[7,10,11,12]}\right) \\
& \left(A_{12,10} A_{8,9} \mathrm{dQ}_{[13,14,15,16],[8,9,10,12]}+\mathrm{dQ}_{[13,14,15,16],[7,9,10,11]} A_{7,9} A_{11,10}+\right. \\
& \left.\mathrm{d} Q_{[13,14,15,16],[7,9,10,12]} A_{12,10} A_{7,9}+\mathrm{d} Q_{[13,14,15,16],[8,9,10,11]} A_{8,9} A_{11,10}\right) / \\
& d A_{[7,8,9],[7,8,9]} \mathrm{d} Q_{[13,14,15,16],[7,8,9,10]} \mathrm{d} Q_{[1,2,3,4],[8,16,17,18]} \mathrm{d} Q_{[13,14,15,16],[9,10,11,12]}
\end{aligned}
$$

Substituting the solutions for $\operatorname{diag}(A)$ into the solutions for the transition probabilities yields a $48=288 / 6$ parameter family of solutions to the $2 \times 2 \times 2$ problem. In two dimensions, there are 64 unknown transition probabilities and a $16=64 / 4$ parameter family of solutions to the $2 \times 2$ problem. Notice that the ratio of unknowns to parameters is higher in three dimensions than it is in two dimensions. The extension of the two dimensional recovery algorithm to $n \times n$ systems gives a $8 n(n-1)$ parameter family of solutions for the $16 n^{2}$ unknown transition probabilities. The analogous extension to the solution of the $2 \times 2 \times 2$ problem will doubtless result in a $O\left(n^{3}\right)$ parameter family of solutions to the $n \times n \times n$ problem. The author's best guess is that the number of parameters will be $24 n^{3}+O\left(n^{2}\right)$. 


\section{Chapter 5}

\section{Conclusion}

Diffuse tomography is still in its infancy, and there are many areas which should be explored. In this thesis a recursive algorithm for computing a $8 n(n-1)$ parameter family of solutions for a $n \times n$ problem was derived in section 3.2. Before deriving this algorithm it was necessary to study consistency conditions in section 3.1. A thorough understanding of the consistency conditions was required in section 3.2.3.2 to reduce the number of parameters in the solution to the modified $4 \times 4$ problem. The recursive recovery algorithm was sketched in section 3.2.4. Finally, the smallest nontrivial problem in three dimensions was considered in Chapter 4. 
As yet unexplored areas which pique the author's interest include completion of a careful study of consistency conditions for the three dimensional model [13]. Understanding the consistency conditions is crucial because the amount and type of additional information required to close the resulting system of equations is directly tied to the number and type of conditions. The very next item on the agenda is to implement the recursive recovery algorithm in three dimensional. The algorithm will be analogous to its two dimensional predecessor. The biggest difference between the two and three dimensional algorithms is complexity. (The three dimensional version will be much worse!) Last, but certainly not least, is a careful stability study of these algorithms. Given a "noiseless" set of data for the two dimensional problem, the recursive algorithm recovers the transition probabilties exactly. Noisy data could introduce large errors. One source of error is inverting $P_{h o}$. When scattering is isotropic, for example, $P_{h o}$ is singular. Another source of error is the fact that the algorithm requires solving nonlinear polynomial systems. Schub and Smale have developed a "condition number" for polynomial systems [15] which could be used in stability studies for both two and three dimensional algorithms. If the recovery algorithms prove to be highly unstable, time-of-flight information would give additional data (we would then have an overdetermined problem) which could be used to reduce errors due to noise.

This thesis work was done on an extremely general Markovian model of photon transport. Neither time-of-flight information nor any physical information about photon transport through tissue were taken into account. A priori information about photon transport can and should be incorporated into this model. (The author doubts that clinicians would find a set of $36 n^{3}$ Markov transition probabilities helpful diagnostic information.) However, the fact that all of the independent data generated by the forward map for this most general model can be recovered indicates (to the author, at least), that data generated by photons which scatter many times contain information independent of the data generated by ballistic photons. 


\section{Bibliography}

[1] F. A. Grünbaum, Tomography with Diffusion, Inverse Problems in Action, ed. P. Sabatier, pp. 16-21, Springer-Verlag, Berlin, 1990.

[2] J. Singer, F. A. Grünbaum, P. Kohn and J. Zubelli, "Image reconstruction of the interior of bodies that diffuse radiation," Science, Vol. 248, 990-993, 1990.

[3] F. A. Grünbaum, "Backscattering comes to the rescue," Contemporary Mathematics, E. Grinberg and E. T. Quinto editors, Vol. 113, 137-239, 1990.

[4] F. A. Grünbaum, "An inverse problem in transport theory: diffuse tomography," Invariant Imbedding and Inverse Problems, SIAM 1993, in Honor of R. Kruger, J. Corones editor, pp.209-215.

[5] F. A. Grünbaum, S. K. Patch, "Analytic inversion of a general model in diffuse tomography," Inverse Problems in Scattering and Imaging, Michael A. Fiddy, Editor, Proc. S.P.I.E. Vol. 1767, pp. 44-54, (1992).

[6] F. A. Grünbaum, S. K. Patch, "Simplification of a general model in diffuse tomography," Photon Migration and Imaging in Random Media, B. Chance, R. Alfano, Editors, Proc. S.P.I.E. 1888, pp. 387-401,(1993).

[7] F. A. Grünbaum, S. K. Patch, "The use of Graßmann Identities for inversion of a general model in diffuse tomography", Proceedings of the Lapland Conference on Inverse Problems, June 14-20, 1992. Saariselkä, Finland.

[8] S. K. Patch, "Consistency Conditions in Diffuse Tomography", Inverse Problems, vol. 10, no. 1, pp. 199-212, (1994). 
[9] W. Feller, An Introduction to Probability Theory and its Applications, $2^{\text {nd }}$ ed., John Wiley and Sons, Inc., New York, 1962.

[10] C. Aitken, Determinants and Matrices, $8^{\text {th }}$ ed., Oliver and Boyd Ltd., Edinburgh, 1954.

[11] Hodge, W. V. D., Pedoe, D., Methods of Algebraic Geometry, Cambridge University Press, 1968.

[12] Griffiths, P., Harris, J., Principles of Algebraic Geometry, Wiley and Sons, Inc., 1978.

[13] S. K. Patch, "A Note on Consistency Conditions in Three Dimensional Diffuse Tomography", Lectures in Applied Mathematics, proceedings of the 1993 AMS-SIAM Summer Conference on Tomography, American Mathematical Society, Providence, RI.

[14] S. L. Primack, C. Chiles, C. E. Putman, "One hundred years of imaging: new benefits, new challenges", Perspectives in Biology and Medicine, Spring 1992, pp. 361-371.

[15] M. Shub, S. Smale, "Complexity of Bezout's Theorem - I., Geometric Aspects", Journal of the American Mathematical Society, 6(1993), no.2, pp. 459-501. Review pending.

[16] J. Alper, "Biomedicine in the Age of Imaging", Science, vol. 261, pp. 554-561, July 30, 1993.

[17] Huang, et al, "Optical Coherence Tomography", Science, vol. 254, pp. 1178-1181, November 22, 1991.

[18] Kristen Leutwyler, "Optical Tomography", Scientific American, pp. 147-9, January 1994.

[19] J. Hebden, D. Delpy, "Infrared lasers muscle in on medical imaging", Physics World, pp. 23-4, August 1993.

[20] D. A. Benaron, D. K. Stevenson, "Optical Time-of-Flight and Absorbance Imaging of Biologic Media", Science, vol. 259, pp. 1463-6, March 51993.

[21] Photon Migration and Imaging in Random Media and Tissues, B. Chance, R. Alfano, Editors, Proc. S.P.I.E. 1888, (1993).

[22] Inverse Problems in Scattering and Imaging, M. A. Fiddy, Editor, Proc. S.P.I.E. 1767, (1992). 

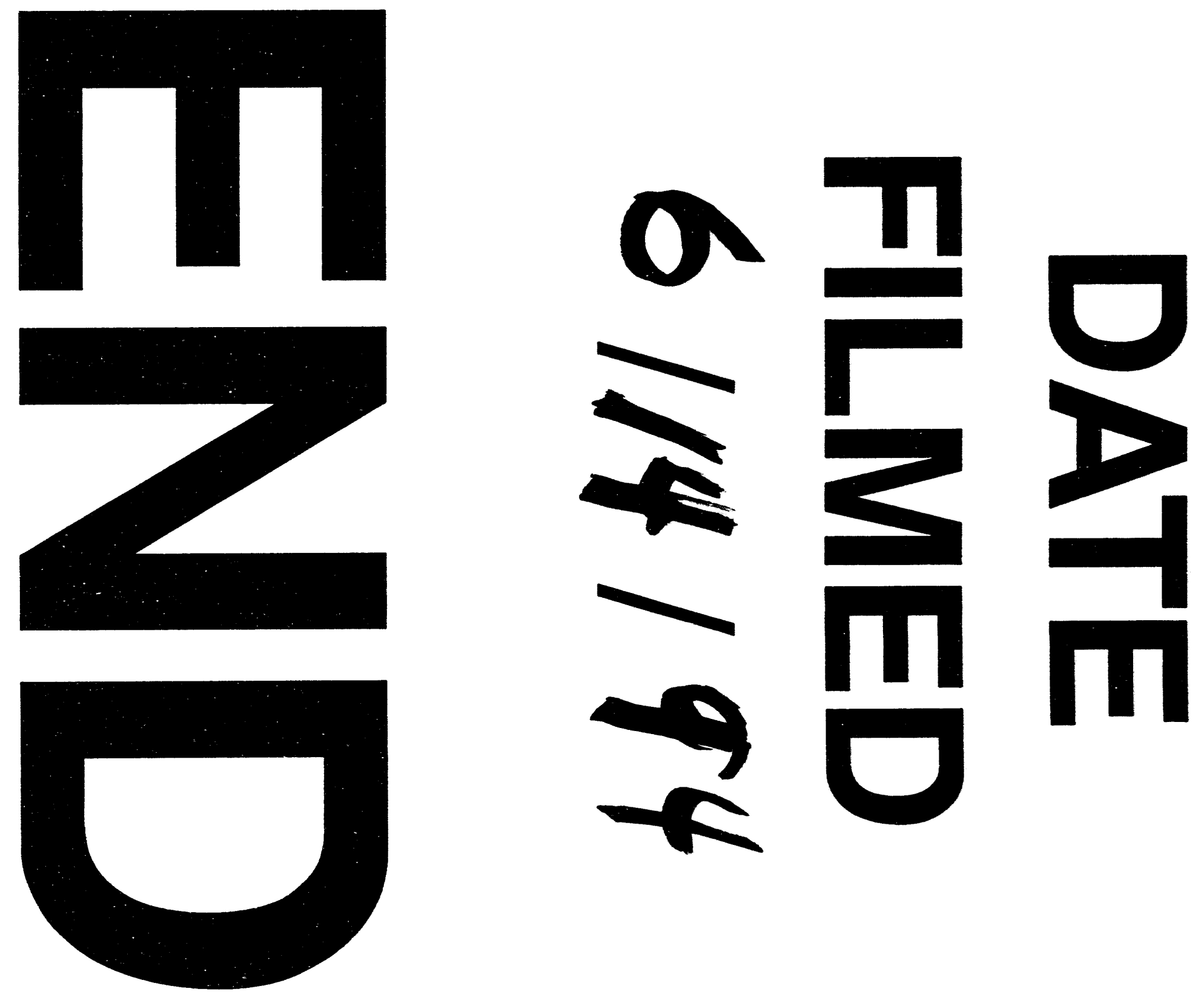
$\longrightarrow$

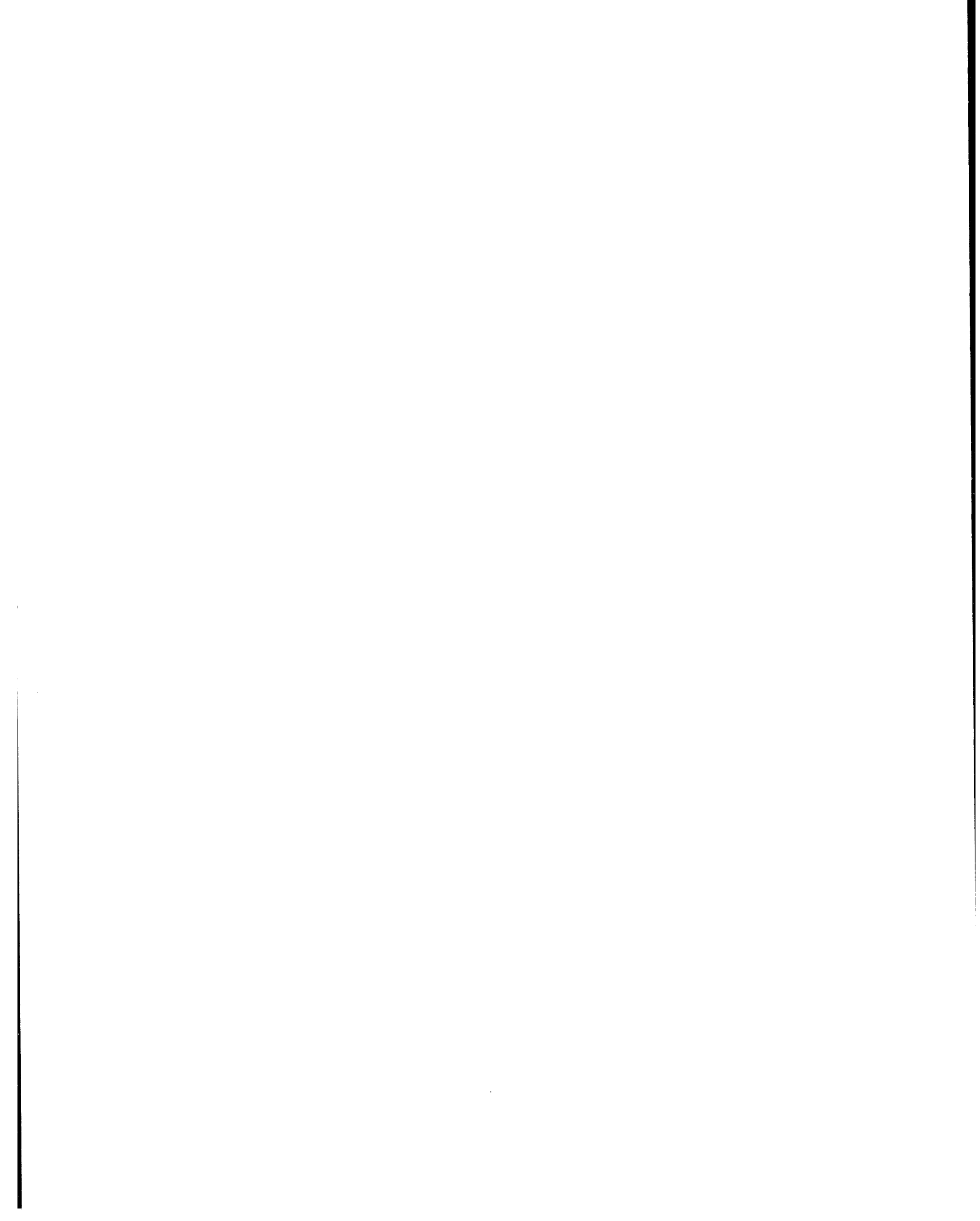

